\title{
Towards incorporating asexually reproducing fungi in the natural classification and notes for pleomorphic genera
}

\author{
Wijayawardene $\mathrm{NN}^{1,2,3}$, Hyde $\mathrm{KD}^{4}$, Anand $\mathrm{G}^{5}$, Dissanayake $\mathrm{LS}^{6}$, Tang $\mathrm{LZ}^{1, *}$ and \\ Dai DQ ${ }^{1, *}$
}

\begin{abstract}
${ }^{1}$ Center for Yunnan Plateau Biological Resources Protection and Utilization, College of Biological Resource and Food Engineering, Qujing Normal University, Qujing, Yunnan 655011, P.R. China

${ }^{2}$ State Key Laboratory of Functions and Applications of Medicinal Plants, Guizhou Medical University, Guiyang 550014, P.R. China

${ }^{3}$ Section of Genetics, Institute for Research and Development in Health and Social Care No: 393/3, Lily Avenue, Off Robert Gunawardane Mawatha, Battaramulla 10120, Sri Lanka ${ }^{4}$ Center of Excellence in Fungal Research, Mae Fah Luang University, Chiang Rai, 57100, Thailand

${ }^{5}$ Department of Botany, University of Delhi, Delhi-110007, India

${ }^{6}$ The Engineering Research Center of Southwest Bio-Pharmaceutical Resource Ministry of Education, Guizhou University, Guiyang 550025, Guizhou Province, P.R. China
\end{abstract}

Wijayawardene NN, Hyde KD, Anand G, Dissanayake LS, Tang LZ, Dai DQ 2021 - Towards incorporating asexually reproducing fungi in the natural classification and notes for pleomorphic genera. Mycosphere 12(1), 238-405, Doi 10.5943/mycosphere/12/1/4

\begin{abstract}
This is a continuation of a series of studies incorporating asexually reproducing fungi in a natural classification. Over 3653 genera (ca. 30,000 morphological species) are known from asexual reproduction (1388 coelomycetes and 2265 hyphomycetes) in their life cycle. Among these, 687 genera are pleomorphic (305 coelomycetous; 378 hyphomycetous and four genera show both coelomycetous and hyphomycetous morphs). We provide notes for these pleomorphic genera in this paper. The 1544 unlinked genera without molecular data (which comprise ca. 3850 species) are listed as Ascomycota genera incertae sedis. It is essential to recollect the fungi which are placed in Ascomycota genera incertae sedis and subject them to DNA based phylogenetic analysis as they might represent new fungal lineages.
\end{abstract}

Keywords - Article 59.1 - Coelomycetous - Hyphomycetous - one fungus one name Pleomorphism

\section{Introduction}

The classification of asexually reproducing fungi belonging to the subkingdom Dikarya (including Ascomycota and Basidiomycota) was a huge challenge in traditional taxonomy. They were traditionally placed in a separate subdivision, Deuteromycotina (Ainsworth 1966). DNA sequence-data is currently being widely used to place fungi in the higher level classification. Tedersoo et al. (2018) suggested that some asexual taxa could represent new fungal lineages. For example, the new phylum Calcarisporiellomycota was introduced by Tedersoo et al. (2018) based on asexual taxon, Calcarisporiella de Hoog. 
Recent outlines and monographs by Wijayawardene et al. (2018, 2020), Hongsanan et al. (2020a, b) and Hyde et al. (2020b) have compiled asexual genera according to their phylogenetic placements. However, a large number of asexual genera (ca. 3850 species in 1544 genera) lack sequence data and thus their placements have not been determined. Holomorph studies are important in mycology, and it was previously determined by cultural studies or cooccurence of both taxa on same substrate. Currently DNA based phylogenetic studies are very useful to link sexual and asexual fungi and this impacts on species nomenclature. In the last decade, several publications which focused on nomenclatural changes (e.g Chapter F, San Juan Chapter F, May et al. 2019) have been published (Table 1).

In this paper, we provide the higher-level classification of asexually producing taxa (genera to class) of Ascomycota and Basidiomycota and specifies whether they show pleomorphism. Notes for pleomorphic genera which have been introduced since 2011 and nomenclatural changes based on San Juan Chapter F are provided (May et al. 2019).

\section{Material \& Methods}

The outline of classification follows Tedersoo et al. (2018), Hongsanan et al. (2020a, b), Hyde et al. (2020b) and Wijayawardene et al. (2020). The generic names are extracted from Kirk et al. (2008, 2013), Wijayawardene et al. (2017a, b, 2018), Index Fungorum (2021) and research articles since 2018 to the end of 2020. Recent name changes of pleomorphic genera (according to San Juan Chapter F) (May et al. 2019) are also incorporated based on articles as in Table 1. However, we included several genera which have been reported with '-like' as in Kirk et al. (2008).

Dubious genera are excluded based on Kirk et al. (2008) and Seifert et al. (2011), but can be added if they are later found to be good genera (legitimate or valid genera). We do not include fossil genera as a separate study is being prepared by Saxena et al. (2021). A question mark before the generic name means that the link between two morphs or the placement of the genus in the higher rank is not confirmed. An asterisk following the names indicates an entry in the "Notes" section.

We have added $(\mathrm{H})=$ hyphomycetous asexual morph, $(\mathrm{C})=$ coelomycetous asexual morph, $(\mathrm{Y})=$ yeast and $(\mathrm{R})=$ Rust against entries where known (Kirk et al. 2008, Seifert et al. 2011, Wijayawardene et al. 2016, 2017a, b, Li et al. 2020). The genera which are reported as pleomorphic genera are indicated by \#. Genera which show synasexual morphs are indicated by $(\mathrm{H})$ and $(\mathrm{C})$.

Table 1 Articles used to update the name changes of pleomorphic genera

\begin{tabular}{ll}
\hline \multicolumn{1}{c}{ Taxonomic group } & \multicolumn{1}{c}{ Reference } \\
\hline Ascomycota & Rossman et al. (2016), Wijayawardene et al. (2017a, b, 2018) \\
Diaporthales & Rossman et al. 2013, 2015b \\
Dothideomycetes & Wijayawardene et al. 2014b, Rossman et al. 2015a, \\
& Hongsanan et al. 2020a, b \\
Leotiomycetes & Johnston et al. 2014 \\
Magnaporthales & Zhang et al. 2016 \\
Orbiliaceae (Orbiliomycetes) & Baral et al. 2017, Ekanayaka et al. 2018 \\
Sordariomycetes & Maharachchikumbura et al. 2015, 2016, \\
& Réblová et al. 2016a, Hyde et al. 2020b \\
Xylariales & Wendt et al. 2017 \\
Xylariomycetidae & Senanayake et al. 2015 \\
Pezizomycetes & Healy et al. 2016 \\
\hline
\end{tabular}

\section{Index to Ascomycota}

Phylum ASCOMYCOTA Caval-Sm.

Subphylum TAPHRINOMYCOTINA O.E. Erikss. \& Winka

Class Taphrinomycetes O.E. Erikss. \& Winka 
Taphrinales Gäum. \& C.W. Dodge

Protomycetaceae Gray

Saitoella Goto et al. 1987 (Y)

Taphrinaceae Gäum.

Lalaria R.T. Moore 1990 (Y)

Subphylum SACHAROMYCOTINA O.E. Erikss. \& Winka

Class Saccharomycetes O.E. Erikss. \& Winka

Saccharomycetales Kudrjanzev

Dipodascaceae Engl. \& E. Gilg

Blastobotrys Klopotek 1967 (H)

Geotrichum Link 1809 (H)

Saprochaete Coker \& Shanor 1939 (H)

Lipomycetaceae E.K. Novák \& Zsolt

Myxozyma Van der Walt et al. 1981 (Y)

Pichiaceae Zender

Brettanomyces N.H. Claussen ex Custers 1940 (Y)

Hyphopichia Arx \& Van der Walt 1976 (Y)

Saccharomycetaceae G. Winter

Grigorovia Gouliamova \& Dimitrov 2020 (Y)

Saccharomycodaceae Kudrjanzev

Kloeckera Janke 1923 (Y)

Trigonopsidaceae M.A. Lachance \& C.P. Kurtzman

Botryozyma Shann \& M.T. Sm. 1992 (Y)

Trigonopsis Schachner 1929 (Y)

Saccharomycetales genera incertae sedis

Aciculoconidium D.S. King \& S.C. Jong 1976 (Y)

Candida Berkhout 1923 (Y)

Macrorhabdus Tomasz. et al. 2003 (Y)

Subphylum PEZIZOMYCOTINA O.E. Erikss. \& Winka

Class Arthoniomycetes O.E. Erikss. \& Winka

Arthoniales Henssen ex D. Hawksw. \& O.E. Erikss.

Arthoniaceae Reichenb. ex Reichenb.

Briancoppinsia Diederich et al. 2012 (C)

Helicobolomyces Matzer 1995 (C)

Reichlingia Diederich \& Scheid. 1996 (H)

Tylophoron Nyl. ex Stizenb. $1862(\mathbf{H})^{\# *}$

Lichenostigmatales Ertz et al.

Phaeococcomycetaceae McGinnis \& Schell

Etayoa Diederich \& Ertz 2014 (C) \#* $^{*}$

Phaeococcomyces de Hoog 1979 (H)

Class Dothideomycetes sensu O.E. Erikss. \& Winka

Subclass Dothideomycetidae P.M. Kirk et al. 
Aureoconidiellales Hern.-Restr. \& Crous

Aureoconidiellaceae Hern.-Restr. \& Crous

Aureoconidiella Hern.-Restr. \& Crous 2020 (H)

Capnodiales Woron.

Aeminiaceae J. Trovão et al.

Aeminium J. Trovão et al. 2019 (H)

Antennulariellaceae Woron.

Antennariella Bat. \& Cif. 1963 (C)

Capnodiaceae (Sacc.) Höhn. ex Theiss.

Capnodium Mont. 1848 (C) \#* $^{\# *}$

Kosmimatamyces Bianchin et al. 2020 (H)

Leptoxyphium Speg. 1918 (H)

Phragmocapnias Theiss. \& Syd. 1918 (C) ${ }^{\# *}$

Euantennariaceae Hughes \& Corlett

Capnokyma S. Hughes 1975 (H)

Hormisciomyces Bat. \& Nascim. 1957 (H)

Plokamidomyces Bat. et al. 1957 (H)

Trichothallus F. Stevens 1925 (H)

Metacapnodiaceae S. Hughes \& Corlett

Capnobotrys S. Hughes 1970 (H)

Capnophialophora S. Hughes 1966 (H)

Metacapnodium Speg. $1918(\mathbf{H})^{\# * *}$

Neoantennariellaceae Abdollahz. \& Crous*

Fumiglobus D.R. Reynolds \& G.S. Gilbert 2006 (C)

Neoantennariella Abdollahz. \& Crous 2020 (C)

Neoasbolisia Abdollahz. \& Crous 2020 (C)

Readerielliopsidaceae Abdollahz. \& Crous

Fumagospora G. Arnaud 1911 (H)

Phaeoxyphiella Bat. \& Cif. 1963 (H)

Readerielliopsis Crous \& Decock 2015 (C)

Scorias Fr. 1832 (C)

Mycosphaerellales (Nannf.) P.F. Cannon

Dissoconiaceae Crous \& de Hoog

Dissoconium de Hoog et al. 1983 (H)

Globoramichloridium Y. Marín \& Crous 2019 (H)

Pseudoveronaea Crous \& Batzer 2012 (H)

Ramichloridium ex de Hoog 1977 (H)

Uwebraunia Crous \& M.J. Wingf. 1996 (H)

Extremaceae Quaedvl. \& Crous

Castanedospora G. Delgado \& A.N. Mill. 2018 (H)

Extremus Quaedvl. \& Crous 2014 (H)

Paradevriesia Crous 2019 (H)

Petrophila de Hoog \& Quaedvl. 2014 (H)

Pseudoramichloridium Cheew. \& Crous 2009 (H) 
Saxophila Selbmann \& de Hoog 2015 (H)

Staninwardia B. Sutton 1971 (C)

Vermiconia Egidi \& Onofri 2014 (H)

Mycosphaerellaceae Lindau

Acervuloseptoria Crous \& Jol. Roux 2014 (C)

Annellosympodiella Crous \& Assefa 2014 (H)

Apseudocercosporella Videira \& Crous 2016 (H)

Asperisporium Maubl. 1913 (H)

Brunswickiella Videira \& Crous 2017 (C)

Camptomeriphila Crous \& M.J. Wingf. 2016 (C)

Caryophylloseptoria Verkley et al. 2013 (C)

Catenulocercospora C. Nakash. et al. 2017 (H)

Cercospora Fresen. 1863 (H)

Cercosporella Sacc. 1880 (H)

Cercosporidium Earle 1901(H)

Chuppomyces Videira \& Crous 2017 (H)

Cladocillium Chun-Hao Chen \& R. Kirschner 2020 (H)

Clarohilum Videira \& Crous $2017(\mathbf{H})^{\# *}$

Clypeosphaerella Guatim. et al. $2017(\mathbf{H})^{\# *}$

Collapsimycopappus A. Hashim. et al. 2018 (H)

Collarispora Videira \& Crous $2017(\mathbf{H})^{\# *}$

Coremiopassalora U. Braun et al. 2017 (H)

Colletogloeum Petr. 1953 (C)

Cytostagonospora Bubák 1916 (C)

Deightonomyces Videira \& Crous 2017 (H)

Devonomyces Videira \& Crous $2017(\mathbf{H})^{\# *}$

Dictyosporina Abreu et al. 2017 (H)

Distocercospora N. Pons \& B. Sutton 1988 (H)

Distocercosporaster Videira et al. 2017 (H)

Distomycovellosiella U. Braun et al. 2017 (H)

Dothistroma Hulbary 1941 (C)

Epicoleosporium Videira \& Crous 2016 (H)

Filiella Videira \& Crous 2016 (H)

Fusoidiella Videira \& Crous 2016 (H)

Graminopassalora U. Braun et al. 2017 (H)

Hyalocercosporidium Videira \& Crous 2017 (H)

Hyalozasmidium U. Braun et al. 2017 (H)

Janetia M.B. Ellis 1976 (H)

Juncomyces Crous 2020 (H)

Lecanosticta Syd. 1922 (C)

Madagascaromyces U. Braun et al. 2017 (H)

Microcyclosporella Jana Frank et al. 2010 (H)

Micronematomyces U. Braun et al. 2017 (H)

Miuraea Hara 1948 (H)

Mycosphaerelloides Videira \& Crous $2016(\mathbf{H})^{\# *}$

Mycovellosiella Rangel 1918 (H)

Neoceratosperma Crous \& Cheew. 2014 (H)

Neocercospora M. Bakhshi et al. 2015 (H)

Neodeightoniella Crous \& W.J. Swart 2013 (H)

Neopenidiella Quaedvl. \& Crous 2014 (H)

Neophloeospora U. Braun et al. 2017 (H) 
Neopseudocercospora Crous 2013 (H)

Neopseudocercosporella Videira \& Crous $2016(\mathbf{H})^{\# *}$

Neoramichloridium Phook. et al. 2017 (H)

Neoseptoria Quaedvl. et al. 2013 (C)

Nothopassalora U. Braun et al. 2017 (H)

Nothopericoniella Videira \& Crous 2017 (H)

Pachyramichloridium Videira \& Crous 2017 (H)

Pallidocercospora Crous 2012 (H) ${ }^{\# * *}$

Pantospora Cif. 1938 (H)

Paracercospora Deighton 1979 (H)

Paracercosporidium Videira \& Crous 2017 (H)

Paramycovellosiella Videira et al. 2017 (H)

Parapallidocercospora Videira et al. $2017(\mathbf{H})^{\# *}$

Passalora Fr. 1849 (H)

Phaeocercospora Crous 2012 (H)

Phaeophleospora Rangel 1916 (C)

Phaeoramularia Munt.-Cvetk. 1960 (H)

Piricauda Bubák 1914 (H)

Phloeospora Wallr. 1833 (C)

Pleopassalora Videira \& Crous 2017 (H)

Pleuropassalora U. Braun et al. 2017 (H)

Pluripassalora Videira \& Crous 2017 (H)

Plurivorosphaerella O. Hassan \& T.H. Chang 2017 (H) \#* $^{\#}$

Polyphialoseptoria Quaedvl. et al. 2013 (C)

Polythrincium Kunze 1817 (H)

Protostegia Cooke 1880 (C)

Pseudocercospora Speg. 1910 (H)

Pseudocercosporella Deighton 1973 (H)

Pseudopericoniella Videira \& Crous 2017 (H)

Pseudophaeophleospora C. Nakash. et al. 2017 (C)

Pseudozasmidium Videira \& Crous $2017(\mathbf{H})^{\# *}$

Ragnhildiana Solheim 1931 (H)

Ramularia Unger 1833 (H) \#* $^{*}$

Ramulariopsis Speg. (H)

Ramulispora Miura 1920 (H)

Rhachisphaerella U. Braun et al. $2017(\mathbf{H})^{\# *}$

Rosisphaerella Videira \& Crous 2017 (H)

Ruptoseptoria Quaedvl. et al. 2013 (C)

Scolecostigmina U. Braun 1999 (H)

Septoria Sacc. 1884 (C)

Sirosporium Bubák \& Serebrian. sensu lato 1912 (H)

Sonderhenia H.J. Swart \& J. Walker 1988 (C)

Sphaerulina Sacc. 1878 (H)

Stromatoseptoria Quaedvl. et al. 2013 (C)

Sultanimyces Videira \& Crous 2017 (H)

Trochophora R.T. Moore 1955 (H)

Uwemyces Hern.-Restr. et al. 2016 (H)

Xenopassalora Crous 2017 (H)

Xenoramularia Videira et al. 2016 (H)

Xenosonderhenia Crous 2012 (C)

Xenosonderhenioides Videira \& Crous 2017 (H)

Zasmidium Fr. 1849 (H) 
Zymoseptoria Quaedvl. \& Crous 2011 (C)

\section{Doubtful pleomorphic genera}

Microcyclus Sacc. et al. 1904 with aposphaeria-like asexual morph (C) $)^{\# *}$

Neodevriesiaceae Quaedvl. \& Crous

Neodevriesia Quaedvl. \& Crous $2014(\mathbf{H})^{\# *}$

Tripospermum Speg. 1918 (H)

Phaeothecoidiellaceae K.D. Hyde \& Hongsanan (= Nowamycetaceae Crous)

Exopassalora Videira \& Crous 2017 (H)

Houjia G.Y. Sun \& Crous 2010 (H)

Phaeothecoidiella Batzer \& Crous 2010 (H)

Sporidesmajora Batzer \& Crous 2010 (H)

Schizothyriaceae Höhn. ex Trotter et al.

Zygophiala E.W. Mason 1945 (H)

Teratosphaeriaceae Crous \& U. Braun

Acidiella Hujslová \& M. Kolařík 2012 (H)

Acidomyces B.J. Baker et al. 2008 (H)

Acrodontium de Hoog 1972 (H)

Apenidiella Quaedvl. \& Crous 2014 (H)

Batcheloromyces Marasas et al. 1975 (H)

Baudoinia J.A. Scott \& Unter. 2007 (H)

Bryochiton Döbbeler \& Poelt 1978 (H) ${ }^{\# *}$

Camarosporula Petr. 1954 (C) ${ }^{\# *}$

Capnobotryella Sugiy. 1987 (H)

Catenulostroma Crous \& U. Braun 2007 (H)

Constantinomyces Egidi \& Onofri 2014 (H)

Davisoniella H.J. Swart 1988 (C)

Devriesia Seifert \& N.L. Nick. 2004 (H)

Elasticomyces Zucconi \& Selbmann 2008 (H)

Eupenidiella Quaedvl. \& Crous 2014 (H)

Friedmanniomyces Onofri 1999 (H)

Hispidoconidioma Tsuneda \& Davey 2010 (H)

Hortaea Nishim. \& Miyaji 1984 (H)

Hyweljonesia R.G. Shivas et al. 2016 (H)

Incertomyces Egidi \& Zucconi 2014 (H)

Lapidomyces de Hoog \& Stielow 2014 (H)

Leptomelanconium Petr. 1923 (C)

Meristemomyces Isola \& Onofri 2014 (H)

Microcyclospora J. Frank et al. 2010 (H)

Monticola Selbmann \& Egidi 2014 (H)

Muriphila Jurjević et al. 2020 (H)

Myrtapenidiella Quaedvl. \& Crous 2014 (H)

Neocatenulostroma Quaedvl. \& Crous 2014 (H)

Neophaeothecoidea Quaedvl. \& Crous 2014 (H)

Neotrimmatostroma Quaedvl. \& Crous 2014 (H)

Oleoguttula Selbmann \& de Hoog 2019 (H)

Parapenidiella Crous \& Summerell 2012 (H)

Penidiella Crous \& U. Braun 2007 (H)

Penidiellomyces Crous et al. 2016 (H) 
Penidiellopsis Sandoval-Denis et al. 2016 (H)

Phaeothecoidea Crous 2007 (H)

Pseudotaeniolina J.L. Crane \& Schokn. 1986 (H)

Queenslandipenidiella Quaedvl. \& Crous 2014 (H)

Readeriella Syd. \& P. Syd. 1908 (C) \#** $^{*}$

Recurvomyces Selbmann \& de Hoog 2008 (H)

Simplicidiella Crous et al. 2016 (H)

Stenella Syd. 1930 (H)

Teratoramularia Videira et al. $2016(\mathbf{H})$

Teratosphaeria Syd. \& P. Syd. 1912 (C) ${ }^{\# *}$

Xanthoriicola D. Hawksw. 1973 (H)

Xenoconiothyrium Crous \& Marinc. 2011 (C)

Xenopenidiella Quaedvl. \& Crous 2014 (H)

Xenophacidiella Crous 2011 (C)

Capnodiales genera incertae sedis

Arthrocatena E. Egidi \& L. Selbmann 2014 (H)

Catenulomyces Egidi \& de Hoog 2019 (H)

Eriosporella Höhn. 1916 (C)

Hyphoconis Egidi \& Quaedvl. 2014 (H)

Neohortaea Quaedvl. \& Crous 2014 (H)

Perusta Egidi \& Stielow 2014 (H)

Pseudoepicoccum M.B. Ellis 1971 (H)

Ramimonilia B. Stielow. \& Quaedvl. 2014 (H)

Ramopenidiella Crous \& R.G. Shivas 2014 (H)

Septocyta Petr. 1927 (C)

Cladosporiales Abdollahz. \& Crous

Cladosporiaceae Nann.

Acroconidiella J.C. Lindq. \& Alippi 1964 (H)

Cladosporium Link $1816(\mathbf{H})^{\# *}$

Graphiopsis Trail 1889 (H)

Rachicladosporium Crous et al. 2007 (H)

Toxicocladosporium Crous \& U. Braun 2007 (H)

Verrucocladosporium K. Schub. et al. 2007 (H)

Comminutisporales Abdollahz. \& Crous

Comminutisporaceae Abdollahz. \& Crous

Comminutispora A.W. Ramaley $1996(\mathbf{H})^{\# *}$

Dothideales Lindau

Dothideaceae Chevall.

Dothiora Fr. 1849 (C) $)^{\# *}$

Endoconidioma Tsuneda et al. 2004 (C)

Kabatina R. Schneid. \& Arx 1966 (C)

Neocylindroseptoria Thambug. \& K.D. Hyde 2014 (C)

Neodothiora Crous et al. 2020 (C)

\section{Neocelosporiaceae Crous}

Celosporium Tsuneda \& Davey 2010 (H)

Neocelosporium Crous 2018 (H) 
Saccotheciaceae Bonord.

Aureobasidium Viala \& G. Boyer 1891 (H)

Kabatiella Bubák 1907 (H)

Moringomyces Crous 2020 (chlamydospore)

Pseudoseptoria Speg. 1910 (C)

Saccothecium Fr. $1836(\text { C })^{\# *}$

Selenophoma Maire 1907 (C)

Dothideales genera incertae sedis

Coniozyma Crous 2008 (C)

Hormonema Lagerb. \& Melin 1927 (H)

Rhizosphaera L. Mangin \& Har. 1907 (C)

Neophaeothecales Abdollahz. \& Crous

Neophaeothecaceae Abdollahz. \& Crous

Neophaeotheca Abdollahz. \& Crous 2020 (H)

Myriangiales Starbäck

Elsinoaceae Höhn. ex Sacc. \& Trotter

Elsinoë Racib. 1900 (C) \#* $^{\# *}$

Myriangiales genera incertae sedis

Gobabebomyces Crous 2020 (C)

Phaeothecales Abdollahz. \& Crous

Phaeothecaceae Darveaux

Phaeotheca Sigler et al. 1981 (H)

Racodiales Abdollahz. \& Crous

Racodiaceae Link

Racodium Fr. 1829 (H)

Subclass Pleosporomycetidae C.L. Schoch et al.

Gloniales Jayasiri \& K.D. Hyde

Gloniaceae (Corda) Boehm et al.

Cenococcum Moug. \& Fr. 1829 (C)

Purpurepithecium Jayasiri \& K.D. Hyde 2017 (H) (** $^{\# *}$

Hysteriales Lindau

Hysteriaceae Chevall.

Gloniopsis De Not. 1847 with aposphaeria-like asexual morph $(\mathbf{C})^{\# *}$

Hysterobrevium E. Boehm \& C.L. Schoch 2009 (C) (** $^{\#}$

Rhytidhysteron Speg. 1881 (C)

Mytilinidiales Boehm et al.

Mytilinidiaceae Kirschst.

Peyronelia Cif. \& Gonz. Frag. 1927 (H)

Pseudocamaropycnis Crous (C)

Septonema Corda 1837 (H)

Taeniolella S. Hughes 1958 (H)

Pleosporales Luttrell ex M.E. Barr 
Acrocalymmaceae Crous \& Trakun.

Acrocalymma Alcorn \& J.A.G. Irwin 1987 (C)

Amniculicolaceae Zhang et al.

Amniculicola Y. Zhang ter \& K.D. Hyde $2008(\mathbf{H})^{\# *}$

Murispora Y. Zhang bis et al. 2009 (C) ${ }^{\# *}$

Vargamyces Tóth 1980 (H)

Amorosiaceae Thambug. \& K.D. Hyde

Amorosia Mantle \& D. Hawksw. 2006 (H)

Angustimassarina Thambug. et al. 2015 (H) ${ }^{\# *}$

Astrosphaeriellaceae Phook. \& K.D. Hyde

Pithomyces Berk. \& Broome 1875 (H)

Aquasubmersaceae A. Hashim. \& Kaz. Tanaka

Aquasubmersa K.D. Hyde \& Huang Zhang (C) ${ }^{\# *}$

Bambusicolaceae D.Q. Dai \& K.D. Hyde

Bambusicola D.Q. Dai \& K.D. Hyde 2012 (C) \#* $^{\#}$

Corylicola Wijesinghe et al. $2020\left(\right.$ C) ${ }^{\# *}$

Leucaenicola Jayasiri et al. 2018 (C)

Palmiascoma Phook. \& K.D. Hyde 2015 (C) (** $^{\#}$

Biatriosporaceae K.D. Hyde

Biatriospora K.D. Hyde \& Borse 1986 (C) (** $^{\# *}$

Camarosporiaceae Wanas. et al.

Camarosporium Schulzer 1870 (C) \#* $^{*}$

Camarosporomyces Crous 2017 (C)

Camarosporidiellaceae Wanas. et al.

Camarosporidiella Wanas. et al. 2017 (C)

Coniothyriaceae W.B. Cooke

Coniothyrium Corda 1840 (C)

Foliophoma Crous 2017 (C)

Neoconiothyrium Crous 2017 (C)

Ochrocladosporium Crous \& U. Braun 2007 (H)

Staurosphaeria Rabenh. 1858 (C) ${ }^{\# *}$

Corynesporascaceae Sivan.

Corynespora Güssow 1906 (H)

Cryptocoryneaceae A. Hashim. \& Kaz. Tanaka

Cryptocoryneum Fuckel 1870 (H)

Cucurbitariaceae G. Winter

Allocucurbitaria Valenz.-Lopez et al. 2017 (C)

Astragalicola Jaklitsch \& Voglmayr 2018 (C)

Cucitella Jaklitsch \& Voglmayr 2018 (C) \#* $^{\text {\# }}$

Cucurbitaria Gray 1821 (C) ${ }^{\# *}$ 
Neocucurbitaria Wanas. et al. 2017 (C) \#* $^{\text {* }}$

Paracucurbitaria Valenz.-Lopez et al. 2017 (C)

Parafenestella Jaklitsch \& Voglmayr 2018 (C) ${ }^{\# *}$

Protofenestella Jaklitsch \& Voglmayr 2018 (C) \#* $^{\text {\# }}$

Syncarpella Theiss. \& Syd. 1915 (C) ${ }^{\# *}$

Synfenestella Jaklitsch \& Voglmayr 2018 (C) ${ }^{\# * *}$

Cyclothyriellaceae Jaklitsch \& Voglmayr

Cyclothyriella Jaklitsch \& Voglmayr 2016 (C) \#* $^{\# *}$

Dictyosporiaceae Boonmee \& K.D. Hyde

Aquadictyospora Z.L. Luo et al. 2017 (H)

Cheirosporium L. Cai \& K.D. Hyde 2008 (H)

Dendryphiella Bubák \& Ranoj. 1914 (H)

Dictyocheirospora D'souza et al. 2016 (H)

Dictyopalmispora Pinruan et al. 2016 (H)

Dictyosporium Corda 1836 (H)

Digitodesmium P.M. Kirk 1981 (H)

Jalapriya D'souza et al. 2016 (H)

Neodendryphiella Iturrieta-González et al. 2018 (H)

Pseudocoleophoma Kaz. Tanaka \& K. Hiray. 2015 (C) (\# $^{\#}$

Pseudoconiothyrium Crous \& R.K. Schumach. 2019 (C)

Pseudodictyosporium Matsush. 1971 (H)

Sajamaea Flakus et al. 2020 (C)

Vikalpa D'souza et al. 2016 (H)

Didymellaceae Gruyter et al. (= Microsphaeropsidaceae Q. Chen et al.)

Allophoma Q. Chen \& L. Cai 2015 (C)

Anthodidymella Phukhams. et al. 2020 (C) $\# *$

Ascochyta Lib. $1830(\mathbf{C})^{\# * *}$

Boeremia Aveskamp et al. $2010\left(\right.$ C) \#* $^{* *}$

Briansuttonomyces Crous 2010 (C)

Calophoma Q. Chen \& L. Cai 2015 (C)

Chaetasbolisia Speg. 1918 (C)

Cumuliphoma Valenz.-Lopez et al. 2017 (C)

Didymella Sacc. ex Sacc. $1880(\mathbf{C})^{\# *}$

Didysimulans Tibpromma et al. $2017(\mathbf{H})^{\# *}$

Dimorphoma L.W. Hou et al. 2020 (C)

Ectodidymella L.W. Hou et al. $2020\left(\right.$ C) ${ }^{\# *}$

Ectophoma Valenz.-Lopez et al. 2017 (C)

Epicoccum Link 1815 (H)

Heterophoma Q. Chen \& L. Cai 2015 (C)

Juxtiphoma Valenzuela-Lopez et al. 2017 (C)

Leptosphaerulina McAlpine 1902 (H) ${ }^{\# *}$

Longididymella L.W. Hou et al. 2020 (C) (** $^{\# *}$

Macroascochyta L.W. Hou et al. 2020 (C)

Microsphaeropsis Höhn. 1917 (C)

Neoascochyta Q. Chen \& L. Cai 2015 (C) \#*

Neodidymelliopsis Q. Chen \& L. Cai 2015 (C) \#* $^{*}$

Neomicrosphaeropsis Thambug. et al. 2016 (C)

Nothophoma Q. Chen \& L. Cai 2015 (C)

Paraboeremia Q. Chen \& L. Cai 2015 (C) ${ }^{\# *}$ 
Paramicrosphaeropsis L.W. Hou et al. 2020 (C)

Phoma Sacc. $1880(\text { C) })^{\# *}$

Phomatodes Q. Chen \& L. Cai 2015 (C)

Pseudoascochyta Valenzuela-Lopez et al. 2016 (C)

Remotididymella Valenzuela-Lopez et al. 2017 (C) ${ }^{\# * *}$

Sclerotiophoma L.W. Hou et al. 2020 (C)

Similiphoma Valenz.-Lopez et al. 2017 (C)

Stagonosporopsis Died. $1912(\mathbf{C})^{\# *}$

Vacuiphoma Valenz.-Lopez et al. 2017 (C)

Vandijckomycella Hern.-Restr. et al. 2020 (C)

Xenodidymella Q. Chen \& L. Cai 2015 (C) \#* $^{*}$

\section{Didymosphaeriaceae Munk}

Alloconiothyrium Verkley et al. 2014 (C)

Austropleospora R.G. Shivas \& L. Morin 2010 (C) (** $^{\text {* }}$

Bimuria D. Hawksw. et al. $1979(\mathbf{C})^{\# *}$

Chromolaenicola Mapook \& K.D. Hyde 2020 (C) \#* $^{\text {* }}$

Cylindroaseptospora Jayasiri et al. 2019 (C)

Didymosphaeria Fuckel $1870\left(\right.$ C) ${ }^{\# *}$

Kalmusia Niessl 1872 (C) ${ }^{\# * *}$

Paracamarosporium Wijayaw. et al. 2014 (C)

Paraconiothyrium Verkley 2004 (C) ${ }^{\# *}$

Paraphaeosphaeria O.E. Erikss. 1967 (C) ${ }^{\# *}$

Pseudocamarosporium Wijayaw. \& K.D. Hyde 2014 (C)

Pseudopithomyces Ariyaw. \& K.D. Hyde 2015 (H)

Spegazzinia Sacc. 1879 (H)

Tremateia Kohlm. et al. $1995(\mathbf{C})^{\# *}$

Verrucoconiothyrium Crous 2015 (C)

Xenocamarosporium Crous \& M.J. Wingf. 2015 (C)

Dothidotthiaceae Crous \& A.J.L. Phillips

Dothidotthia Höhn. 1918 (H) ${ }^{\# *}$

Mycocentrospora Deighton 1972 (H)

Phaeomycocentrospora Crous et al. 2012 (H)

Pleiochaeta (Sacc.) S. Hughes 1951 (H)

Thyrostroma Höhn. 1911 (H)

Wilsonomyces Adask. et al. 1990 (H)

Fuscostagonosporaceae Jayasiri et al.

Fuscostagonospora Kaz. Tanaka \& K. Hiray. 2015 (C) \#* $^{\#}$

\section{Fusculinaceae Crous}

Fusculina Crous \& Summerell 2006 (C)

Gordonomyces Crous \& Marinc. 2011 (C)

Halojulellaceae Suetrong et al.

Halojulella Suetrong et al. 2013 (C) \#* $^{* *}$

Omania Maharachch. et al. 2020 (C)

Hermatomycetaceae Locq. ex A. Hashim. \& Kaz. Tanaka Hermatomyces Speg. 1910 (H) 
Hypsostromataceae Huhndorf

Hypsostroma Huhndorf (C) ${ }^{\# *}$

\section{Latoruaceae Crous}

Latorua Crous 2015 (H)

Matsushimamyces Rahul Sharma \& Rohit Sharma 2015 (H) (nom. illegit.)

Polyschema H.P. Upadhyay 1966 (H)

Pseudoasteromassaria M. Matsum. \& Kaz. Tanaka 2016 (C) $)^{\# *}$

Triseptata Boonmee \& Phookamsak $2020(\mathbf{H})^{\# *}$

Lentimurisporaceae N.G. Liu et al.

Bahusandhika Subram. 1956 (H)

Lentimurispora N.G. Liu et al. 2019 (H)

Lentitheciaceae Yin. Zhang et al.

Lentithecium K.D. Hyde et al. 2009 (C) ${ }^{\# *}$

Murilentithecium Wanas. et al. 2014 (C) ${ }^{\# *}$

Phragmocamarosporium Wijayaw. et al. 2015 (C)

Pleurophoma Höhn. 1914 (C)

Setoseptoria Quaedvl. 2013 (C)

Towyspora Wanas. et al. $2016(\mathbf{C})^{\# *}$

Leptosphaeriaceae M.E. Barr

Alternariaster E.G. Simmons 2007 (H)

Chaetoplea (Sacc.) Clem. 1931 (C) \#** $^{\# *}$

Heterosporicola Crous 2017 (C)

Leptosphaeria Ces. \& De Not. $1863(\mathbf{C})^{\# *}$

Longiseptatispora L.W. Hou \& Crous

Ochraceocephala Voglmayr \& Aiello (H)

Paraleptosphaeria Gruyter et al. 2012 (= Acicuseptoria Quaedvl. et al. 2013 fide Hongsanan et al. 2020a) (C) \#* $^{\# *}$

Plenodomus Preuss $1851(\mathbf{C})^{\# *}$

Pseudoleptosphaeria Ariyaw. \& K.D. Hyde 2015 (C)

Querciphoma Crous 2017 (C)

Sclerenchymomyces Phukhams. \& K.D. Hyde 2020 (C) \#* $^{*}$

Sphaerellopsis Cooke 1883 (C)

Subplenodomus Gruyter et al. 2012 (C)

\section{Libertasomycetaceae Crous}

Libertasomyces Crous \& Roets 2016 (C)

Neoplatysporoides Crous \& M.J. Wingf. 2015 (C) (** $^{\# *}$

Lindgomycetaceae K. Hiray. et al.

Clohesyomyces K.D. Hyde 1993 (C)

Lolia Abdel-Aziz \& Abdel-Wahab 2010 (C)

Longiostiolaceae Phukhams. et al.

Shearia Petr. 1924 (C)

Longipedicellataceae Phukhams. et al.

Longipedicellata $\mathrm{H}$. Zhang et al. $2016(\mathbf{H})^{\# *}$

Pseudoxylomyces Kaz. Tanaka \& K. Hiray. 2015 (H) 
Submersispora W. Dong, H. Zhang \& K.D. Hyde 2020 (H)

Lophiostomataceae Sacc.

Coelodictyosporium Thambug. \& K.D. Hyde 2015 (C) (** $^{\# *}$

Crassiclypeus A. Hashim. et al. 2018 (C) ${ }^{\# *}$

Desertiserpentica Maharachch. et al. 2020 (C)

Dimorphiopsis Crous 2013 (C)

Flabellascoma A. Hashim. et al. $2018(\text { C })^{\# *}$

Lophiostoma Ces. \& De Not. 1863 (C) \#* Magnopulchromyces L.B. Conc. et al. 2020 (H)

Pseudopaucispora A. Hashim. 2018 (C) (** $^{\# *}$

Lophiotremataceae K. Hiray. \& Kaz. Tanaka

Atrocalyx A. Hashim. \& Kaz. Tanaka 2017 (C) ${ }^{\# *}$

Crassimassarina A. Hashim. \& Kaz. Tanaka 2017 (C) \#* $^{\text {* }}$

Cryptoclypeus A. Hashim. \& Kaz. Tanaka 2017 (C) ${ }^{\# *}$

Galeaticarpa A. Hashim. \& Kaz. Tanaka 2017 (C) \#* $^{\# *}$

Lophiotrema Sacc. $1878(\mathbf{C})^{\# *}$

Pseudocryptoclypeus A. Hashim. \& Kaz. Tanaka 2017 (C) (** $^{*}$

Macrodiplodiopsidaceae Voglmayr et al.

Macrodiplodiopsis Petr. 1922 (C) \#* $^{*}$

Pseudochaetosphaeronema Punith. 1979 (C)

Massarinaceae Munk

Helminthosporium Link 1809 (= Helminthosporiella Hern.-Restr. et al. 2016) (H) ${ }^{\# *}$

Massarina Sacc. $1883(\mathbf{C})^{\# *}$

Pseudodidymosphaeria Thambug. \& K.D. Hyde 2015 (C) (** $^{\# *}$

Pseudosplanchnonema Chethana et al. 2015 (C) ${ }^{\# *}$

Stagonospora (Sacc.) Sacc. 1884 (C)

Suttonomyces Wijayaw. et al. 2015 (C)

Melanommataceae $\mathrm{G}$. Winter

Aposphaeria Sacc. 1880 (C)

Exosporiella P. Karst. $1892(\mathbf{H})^{\# *}$

Fusiconidium J.F. Li et al. 2017 (H)

Melanocamarosporioides D. Pem et al. 2018 (C)

Melanocamarosporium Wijayaw. et al. 2016 (C)

Melanodiplodia Wanas. et al. 2018 (C)

Melanomma Nitschke ex Fuckel $1870(\mathbf{C})^{\# *}$

Muriformistrickeria Q. Tian et al. $2015(\text { C })^{\# *}$

Petrakia Syd. \& P. Syd. 1913 (H) ${ }^{\# *}$

Phragmocephala E.W. Mason \& S. Hughes 1951 (H)

Phragmotrichum Kunze 1823 (C)

Pleotrichocladium Hern.-Restr. et al. 2017 (H)

Praetumpfia Jaklitsch \& Voglmayr 2017 (C) ${ }^{\# *}$

Pseudodidymella C.Z. Wei et al. 1997 (H) ${ }^{\# *}$

Seifertia Partr. \& Morgan-Jones 2002 (H)

Tumularia Descals \& Marvanová 1987(H)

Uzbekistanica Wanas. et al. 2018 (C) \#* $^{\text {\#* }}$

Xenostigmina Crous 1998 (H)

Morosphaeriaceae Suetrong et al. 2009 
Neohelicascus W. Dong et al. $2020\left(\right.$ C) \#* $^{\# *}$

Neocamarosporiaceae Wanas. et al.

Neocamarosporium Crous \& M.J. Wingf. 2014 (C) \#* $^{\# *}$

Neohendersoniaceae Giraldo \& Crous

Medicopsis Gruyter et al. 2012 (C)

Neohendersonia Petr. 1921 (C)

Neomedicopsis Crous \& Akulov. 2019 (C)

Neophaeosphaeriaceae Ariyaw. et al.

Neophaeosphaeria M.P.S. Câmara et al. 2003 (C) \#* $^{\# *}$

Neopyrenochaetaceae Valenz.-Lopez et al.

Neopyrenochaeta Valenz.-Lopez et al. 2017 (C)

Neomassarinaceae Mapook \& K.D. Hyde

Pseudohelminthosporium Phukhams. \& K.D. Hyde 2020 (H)

Nigrogranaceae Jaklitsch \& Voglmayr

Nigrograna Gruyter et al. 2012 (C) ${ }^{\# *}$

Occultibambusaceae D.Q. Dai \& K.D. Hyde

Brunneofusispora S.K. Huang \& K.D. Hyde 2019 (C) (** $^{\# *}$

Occultibambusa D.Q. Dai \& K.D. Hyde 2016 (C) ${ }^{\# *}$

Seriascoma Phook. et al. 2016 (C) ${ }^{\# *}$

Versicolorisporium Sat. Hatak. et al. 2008 (C)

Ohleriaceae Jaklitsch \& Voglmayr

Ohleria Fuckel. 1868 (C) ${ }^{\# *}$

Parabambusicolaceae Kaz. Tanaka \& K. Hiray.

Parabambusicola Kaz. Tanaka \& K. Hiray. 2015 (H) ${ }^{\# *}$

Paramonodictys N.G. Liu et al. 2020 (H)

Pseudomonodictys Doilom et al. 2015 (H)

Paradictyoarthriniaceae Doilom et al.

Paradictyoarthrinium Matsush. 1996 (H)

Parapyrenochaetaceae Valenz.-Lopez et al.

Parapyrenochaeta Valenz.-Lopez et al. 2017 (C)

Quixadomyces Cantillo \& Gusmão 2018 (C)

Periconiaceae (Sacc.) Nann.

Noosia Crous et al. 2011 (H)

Periconia Tode $1791(\mathbf{H})^{\# *}$

Phaeoseptaceae S. Boonmee et al.

Pleopunctum N.G. Liu et al. 2019 (H)

Phaeosphaeriaceae M.E. Barr

Amarenographium O.E. Erikss. 1982 (C)*

Ampelomyces Ces. ex Schltdl. 1852 (C) 
Banksiophoma Crous 2017 (C)

Bhagirathimyces S.M. Singh \& S.K. Singh 2020 (H)

Bhatiellae Wanas. et al. 2018 (H)

Camarosporioides W.J. Li \& K.D. Hyde 2016 (C)

Chaetosphaeronema Moesz 1915 (C)

Didymocyrtis Vain. 1921 (C) \#* $^{*}$

Dlhawksworthia Wanas. et al. 2018 (C) \#* $^{*}$

Edenia M.C. González et al. 2007 (H)

Hydeomyces Maharachch. et al. 2019 (C) ${ }^{\# *}$

Lecanostictopsis B. Sutton \& Crous 1997 (H)

Mauginiella Cavara 1925 (H)

Melnikia Wijayaw. et al. 2016 (C)

Murichromolaenicola Mapook \& K.D. Hyde 2020 (H) ${ }^{\# *}$

Muriphaeosphaeria Phukhams. et al. 2016 (C) \#* $^{*}$

Neosetophoma Gruyter et al. 2010 (C)

Neosphaerellopsis Crous \& Trakun. 2014 (C)

Neostagonospora Quaedvl. et al. 2013 (C)

Neostagonosporella C.L. Yang et al. $2019(\mathbf{C})^{\# *}$

Neosulcatispora Crous \& M.J. Wingf. 2015 (C)

Paraphoma Morgan-Jones \& J.F. White 1983 (C)

Parastagonospora Quaedvl. et al. 2013 (C)

Parastagonosporella M. Bakhshi et al. 2018 (C)

Phaeopoacea Thambug. et al. 2017 (C) ${ }^{\# *}$

Phaeosphaeria I. Miyake 1909 (C) \#*

Phaeoseptoriella Crous 2019 (C)

Phaeosphaeriopsis M.P.S. Câmara et al. 2003 (C) \#* $^{\text {* }}$

Phaeostagonospora A.W. Ramaley 1997 (C)

Piniphoma Crous \& R.K. Schumach. 2019 (C)

Poaceicola W.J. Li et al. 2015 (C) \#* $^{-1}$

Populocrescentia Wanas. et al. $2015(\mathbf{H})^{\# *}$

Pseudostaurosphaeria Mapook \& K.D. Hyde 2020 (C)

Sclerostagonospora Höhn. 1917 (C)

Scolicosporium Lib. ex Roum. 1880 (C)

Septoriella Oudem. 1889 (C)

Setophoma Gruyter et al. 2010 (C)

Tiarospora Sacc. \& Marchal 1885 (C)

Tintelnotia S.A. Ahmed et al. 2017 (C)

Vagicola K.W.T. Chethana \& K.D. Hyde 2015 (H) ${ }^{\# *}$

Vrystaatia Quaedvl. et al. 2013 (C)

Wojnowiciella Crous et al. 2015 (C)

Xenophoma Crous \& Trakun. 2014 (C)

Xenoseptoria Quaedvl. et al. 2013 (C)

Yunnanensis Karun. et al. 2017 (C) ${ }^{\# *}$

Pleomassariaceae M.E. Barr

Beverwykella Tubaki 1975 (H)

Myxocyclus Riess 1852 asexual morph of Splanchnonema sensu lato $(\mathbf{C})^{\# *}$

Prosthemium Kunze 1817 (C) (** $^{*}$

Pleomonodictydaceae Hern.-Restr. et al.

Pleomonodictys Hern.-Restr. et al. 2017 (H)

Pleohelicoon Jayasiri et al. 2019 (H) 
Pleosporaceae Nitschke

Alternaria Nees $1816(\mathbf{H})^{\# *}$

Bipolaris Shoemaker $1959(\mathbf{H})^{\# *}$

Clathrospora Rabenh. $1857(\mathbf{H})^{\# *}$

Comoclathris Clem. 1909 (C) \#* $^{\# *}$

Curvularia Boedijn 1933 (H) ${ }^{\# *}$

Dichotomophthora Mehrl. \& Fitzp. ex M.B. Ellis 1971 (H)

Exserohilum K.J. Leonard \& Suggs $1974(\mathbf{H})^{\# *}$

Gibbago E.G. Simmons 1986 (H)

Johnalcornia Y.P. Tan \& R.G. Shivas 2014 (H)

Paradendryphiella Woudenb. \& Crous 2013 (H)

Pleoseptum A.W. Ramaley \& M.E. Barr 1995 (C) \#* $^{\# *}$

Porocercospora Amaradasa et al. 2014 (H)

Pyrenophora Fr. 1849 (H) ${ }^{\# *}$

Stemphylium Wallr. $1833(\mathbf{H})^{\# *}$

Tamaricicola Thambug. et al. $2016(\mathbf{C})^{\# *}$

Pseudoastrosphaeriellaceae Phook. \& K.D. Hyde

Pseudoastrosphaeriella Phook. et al. 2015 (C) \#* $^{*}$

Pseudoberkleasmiaceae Phukhams. \& K.D. Hyde

Pseudoberkleasmium Tibpromma \& K.D. Hyde 2018 (H)

Pseudocoleodictyosporaceae Doilom \& K.D. Hyde

Pseudocoleodictyospora Doilom \& K.D. Hyde 2016 (H)

Subglobosporium Doilom \& K.D. Hyde 2016 (H)

Pseudopyrenochaetaceae Valenz.-Lopez et al.

Pseudopyrenochaeta Valenzuela-Lopez et al. 2017 (C)

Pyrenochaetopsidaceae Valenz.-Lopez et al

Pyrenochaetopsis Gruyter et al. 2010 (C)

Neopyrenochaetopsis Valenz-Lopez et al. 2017 (C)

Xenopyrenochaetopsis Valenz.-Lopez et al. 2017 (C)

Roussoellaceae J.K. Liu et al.

Cytoplea Bizz. \& Sacc. 1885 (C)

Immotthia M.E. Barr 1987 (C) (** $^{\#}$

Neoroussoella Jian K. Liu et al. $2014(\mathbf{C})^{\# *}$

Pararoussoella Wanas. et al. 2018 (C) ${ }^{\# *}$

Roussoella Sacc. 1888 (C) ${ }^{\# *}$

Pseudoneoconiothyrium Wanas. et al. 2018 (C)

Pseudoroussoella Mapook \& K.D. Hyde 2020 (C) \#* $^{*}$

Roussoellopsis I. Hino \& Katum. 1965 (C) (** $^{*}$

Shiraiaceae Y.X. Liu et al.

Neoshiraia H.A. Ariyaw. 2020 (C)

Rubroshiraia D.Q. Dai \& K.D. Hyde 2019 (C) \#* $^{\# *}$

Shiraia Henn. 1900 (C) \#* $^{\# *}$

Sporormiaceae Munk

Forliomyces Phukhams. et al. 2016 (C) 
Sparticola Phukhams. et al. $2016(\mathbf{H})^{\# *}$

Trichophoma Magaña-Dueñas et al. 2020 (H)

Westerdykella Stolk 1955 (C) ${ }^{\# *}$

Xenomonodictys Hern.-Restr. et al. 2020 (H)

Sulcatisporaceae Kaz. Tanaka \& K. Hiray.

Anthosulcatispora Phukhams. \& K.D. Hyde 2020 (C) (** $^{\# *}$

Loculosulcatispora G.C. Ren \& K.D. Hyde 2020 (C)

Magnicamarosporium Kaz. Tanaka \& K. Hiray. 2015 (C)

Neobambusicola Crous \& M.J. Wingf. 2014 (C)

Pseudobambusicola Hern.-Restr. \& Crous 2018 (C)

Sulcatispora Kaz. Tanaka \& K. Hiray. 2015 (C) $)^{\# *}$

Teichosporaceae M.E. Barr

Floricola Kohlm. \& Volkm.-Kohlm. 2000 (C)*

Magnibotryascoma Thambug. \& K.D. Hyde 2015 (C) (** $^{\# *}$

Paulkirkia Wijayaw. et al. 2016 (C)

Teichospora Fuckel $1870(\mathbf{C})^{\# *}$

Tetraplosphaeriaceae Kaz. Tanaka \& K. Hiray.

Polyplosphaeria Kaz. Tanaka \& K. Hiray. 2009 (H) ${ }^{\# *}$

Pseudotetraploa Kaz. et al. 2009 (H)

Quadricrura Kaz. Tanaka et al. 2009 (H)

Shrungabeeja V.G. Rao \& K.A. Reddy 1981 (H)

Tetraploa Berk. \& Broome $1850(\mathbf{H})^{\# *}$

Triplosphaeria Kaz. Tanaka \& K. Hiray. 2009 (H) ${ }^{\# *}$

Thyridariaceae Sacc.

Cycasicola Wanas. et al. 2018 (C)

Liua Phook. \& K.D. Hyde 2019 (C)

Parathyridariella Prigione et al. 2020 (H)

\section{Torulaceae Corda}

Dendryphion Wallr. 1833 (H)

Neotorula Ariyaw. et al. 2016 (H)

Rostriconidium Z.L. Luo et al. 2018 (H)

Rutola J.L. Crane \& Schokn. 1978 (H)

Sporidesmioides J.F. Li et al. 2016 (H)

Torula Pers. 1795 (H)

Trematosphaeriaceae K.D. Hyde et al.

Trematosphaeria Fuckel 1870 (C) $)^{\# *}$

Tzeananiaceae H.A. Ariyaw. et al.

Tzeanania Ariyaw. et al. 2018 (C)

Zopfiaceae G. Arnaud ex D. Hawksw. 1992

Zopfia Rabenh. 1874 (H) ${ }^{\# *}$

Pleosporales genera incertae sedis

Aegeanispora E.B.G. Jones \& Abdel-Wahab 2017 (C)

Antealophiotrema A. Hashim. \& Kaz. Tanaka 2017 (H) ${ }^{\# *}$ 
Briansuttonia R.F. Castañeda et al. 2004 (H)

Camarographium Bubák 1916 (C)

Cerebella Ces. 1851 (H)

Chaetodiplodia P. Karst. 1884 (C)

Chaetophoma Cooke 1878 (C)

Cheiromoniliophora Tzean \& J.L. Chen 1990 (H)

Crassiperidium M. Matsum. \& Kaz. Tanaka 2018 (C) ${ }^{\# * *}$

Cyclothyrium Petr. 1923 (C)

Dokmaia I. Promputtha 2003 (H)

Megacapitula J.L. Chen \& Tzean 1993 (H)

Neocurreya Thambug. \& K.D. Hyde 2017 (C) (** $^{*}$

Paraepicoccum Matsush. 1993 (H)

Parameliola Hongsanan et al. 2016 (H)

Perthomyces Crous 2017 (C)

Phialophorophoma Linder 1944 (C)

Pseudohendersonia Crous \& M.E. Palm 1999 (C)

Pyrenochaeta De Not. 1849 (C)

Repetophragma Subram. 1992 (H)

Scleroramularia Batzer \& Crous 2011 (H)

Scolecobasidium E.V. Abbott 1927 (H)

Setophaeosphaeria Crous \& Y. Zhang ter 2014 (C) ${ }^{\# *}$

Sirodesmium De Not. 1849 (C)

Spiroplana Voglmayr et al. 2011 (H)

Dothideomycetes orders incertae sedis

Abrothallales Pérez-Ort. \& Suija

Lichenoconiaceae Diederich \& Lawrey

Lichenoconium Petr. \& Syd. 1927 (C)

Acrospermales Minter et al.

Acrospermaceae Fuckel

Acrospermum Tode 1783 with dactylaria-like asexual morph $(\mathbf{H})^{\# *}$

Gonatophragmium Deighton 1969 (H)

Acrospermales genus incertae sedis

Pseudovirgaria H.D. Shin et al. 2007 (H)

Asterinales M.E. Barr ex D. Hawksw. \& O.E. Erikss.

Asterinaceae Hansf.

Asterinella Theiss. 1912 with asteromella-like asexual morph $(\mathbf{C})^{\# *}$

Prillieuxina G. Arnaud 1918 (C) ${ }^{\# *}$

Uleothyrium Petr. 1929 (C) (** $^{\# *}$

Asterinales genera incertae sedis

Discopycnothyrium Hongsanan \& K.D. Hyde 2017 (C)

Pirozynskiella S. Hughes 2007 (H)

Botryosphaeriales C.L. Schoch et al.

Aplosporellaceae Slippers et al.

Alanomyces Roh. Sharma 2017 (C)

Aplosporella Speg. 1880 (C) 
Botryosphaeriaceae Theiss. \& H. Syd.

Alanphillipsia Crous \& M.J. Wingf. 2013 (C)

Barriopsis A.J.L. Phillips et al. 2008 (C)

Botryobambusa Phook. et al. 2012 (C) ${ }^{\# *}$

Botryosphaeria Ces. \& De Not. 1863 (C) ${ }^{\# *}$

Cophinforma Doilom et al. $2012\left(\right.$ C) ${ }^{\# *}$

Diplodia Fr. $1834(\mathbf{C})^{\# *}$

Dothiorella Sacc. 1880 (= Spencermartinsia A.J.L. Phillips et al. 2008) (C) ${ }^{\# *}$

Endomelanconiopsis E.I. Rojas \& Samuels 2008 (C)

Eutiarosporella Crous 2015 (C) $^{\# *}$

Lasiodiplodia Ellis \& Everh. 1896 (C) (* $^{\#}$

Macrophomina Petr. 1923 (C)

Marasasiomyces Crous 2015 (C)

Microdiplodia Allesch. 1901 (C)

Mucoharknessia Crous et al. 2015 (C)

Neodeightonia C. Booth $1970(\mathbf{C})^{\# *}$

Neofusicoccum Crous et al. 2006 (C) ${ }^{\# *}$

Neoscytalidium Crous \& Slippers 2006 (C)

Oblongocollomyces Tao Yang \& Crous 2017 (C)

Phaeobotryon Theiss. \& Syd. 1915 (C) ${ }^{\# *}$

Sakireeta Subram. \& K. Ramakr. 1957 (C)

Sardiniella Linaldeddu et al. 2016 (C)

Sphaeropsis Sacc. 1880 (C) \#* $^{2}$

Tiarosporella Höhn. 1919 (C)

Melanopsaceae Phillips et al.

Melanops Nitschke $1870(\text { C) })^{\# *}$

Phyllostictaceae Fr. (= Pseudofusicoccumaceae Tao Yang \& Crous)

Phyllosticta Pers. 1818 (C) (** $^{*}$

Pseudofusicoccum Mohali et al. 2006 (C)

Planistromellaceae M.E. Barr

Kellermania Ellis \& Everh. $1885\left(\right.$ C) ${ }^{\# *}$

Umthunziomyces Crous \& M.J. Wingf. 2016 (C)

Saccharataceae Slippers et al. (= Septorioideaceae Wyka \& Broders)

Neoseptorioides Crous et al. 2015 (C)

Pileospora Tanney \& Seifert 2019 (C)

Saccharata Denman \& Crous 2004 (C) \#*

Septorioides Quaedvl. et al. 2013 (C)

Cladoriellales Crous

Cladoriellaceae Crous

Cladoriella Crous 2006 (H)

Collemopsidiales Pérez-Ortega

Xanthopyreniaceae Zahlbr.

Collemopsidium Nyl. 1881 (C)

Eremomycetales Pem \& Hyde

Eremomycetaceae Malloch \& Cain 
Eremomyces Malloch \& Cain $1971(\mathbf{C})^{\# *}$

Eremomycetales genera incertae sedis

Arthrographis G. Cochet ex Sigler \& J.W. Carmich. 1976 (H)

Jahnulales Pang et al.

Aliquandostipitaceae Inderbitzin

Brachiosphaera Nawawi 1976 (H)

Xylomyces Goos et al. 1977 (H)

Kirschsteiniotheliales Hern.-Restr. et al.

Kirschsteiniotheliaceae S. Boonmee \& K.D. Hyde

Kirschsteiniothelia D. Hawksw. 1985 (H) ${ }^{\# *}$

Kirschsteiniotheliales genera incertae sedis

Brachysporiella Bat. 1952 (Brachysporiella sensu lato) (H)

Microthyriales G. Arnaud

Microthyriaceae Sacc.

Neoanungitea Crous 2017 (H)

Pseudopenidiella Crous \& Koukol 2012 (H)

Microthyriales genera incertae sedis

Heliocephala V.G. Rao et al. 1984 (H)

Neoscolecobasidium Crous 2016 (H)

Parazalerion Madrid et al. 2019 (H)

Minutisphaerales Raja et al.

Acrogenosporaceae Jayasiri \& K.D. Hyde

Acrogenospora M.B. Ellis $1971(\mathbf{H})^{\# *}$

Monoblastiales Lücking et al.

Monoblastiaceae Walt. Watson

Acrocordia A. Massal. 1854 (C) ${ }^{\# *}$

Anisomeridium (Müll. Arg.) M. Choisy 1928 (C) \#* $^{\text {\# }}$

Caprettia Bat. \& H. Maia 1965 (C) \#* $^{*}$

Funbolia Crous \& Seifert 2011 (H)

Italiofungus Crous 2020 (C)

Megalotremis Aptroot 1991 (C) ${ }^{\# *}$

Monoblastia Riddle 1923 (C) \#*

Pseudopassalora Crous 2011 (H)

Trypetheliopsis Asahina 1937 (C) ${ }^{\# *}$

Murramarangomycetales Crous

Murramarangomycetaceae Crous

Murramarangomyces Crous 2017 (H)

Muyocopronales Mapook et al.

Muyocopronaceae K.D. Hyde

Arxiella Papendorf 1967 (H)

Leptodiscella Papendorf 1969 (H)

Paramycoleptodiscus Crous \& M.J. Wingf. 2016 (C) 
Mycoleptodiscus Ostaz. 1968 (H)

Neocochlearomyces Pinruan et al. 2019 (H)

Neomycoleptodiscus Pinruan et al. 2019 (H)

Stigmatodiscales Voglmayr \& Jaklitsch

Stigmatodiscaceae Voglmayr \& Jaklitsch

Stigmatodiscus Voglmayr \& Jaklitsch 2016 (= Asterodiscus Voglmayr et al. 2016) (C) ${ }^{\# *}$

Strigulales Lücking et al.

Strigulaceae Zahlbr

Dichoporis Clem. 1909 (C) $)^{\# *}$

Flagellostrigula Lücking et al. 2020 (C)*

Flavobathelium Lücking et al. 1997 (C) ${ }^{\# *}$

Phyllobathelium (Müll. Arg.) Müll. Arg. 1890 (C) (** $^{*}$

Phyllocharis Fée $1825\left(\right.$ C) ${ }^{\# *}$

Phylloporis Clem. 1984 (C) \#* $^{*}$

Puiggariella Speg. 1881 (C) $)^{\# *}$

Raciborskiella Höhnel 1909 (C) ${ }^{\# *}$

Racoplaca Fée $1825(\mathbf{C})^{\# *}$

Serusiauxiella S.H. Jiang et al. $2020(\text { C })^{\# *}$

Strigula Fr. $1823(\mathbf{C})^{\# *}$

Tenuitholiascaceae S.H. Jiang et al.

Tenuitholiascus S.H. Jiang et al. 2020 (C) (** $^{\#}$

Superstratomycetales van Nieuwenhuijzen et al.

Superstratomycetaceae van Nieuwenhuijzen et al.

Superstratomyces van Nieuwenhuijzen et al. 2017 (C)

Trypetheliales Lücking et al.

Trypetheliaceae Zenker

Astrothelium Eschw. $1824(\mathbf{C})^{\# *}$

Bogoriella Zahlbr. 1928 (C) ${ }^{\# *}$

Dictyomeridium Aptroot et al. 2016 (C) \#* $^{\text {* }}$

Nigrovothelium Lücking et al. 2016 (C) ${ }^{\# * *}$

Polymeridium (Müll. Arg.) R.C. Harris 1980 (C) ${ }^{\# *}$

Pseudobogoriella Lücking et al. 2020 (C) (** $^{\# *}$

Pseudopyrenula Müll. Arg. 1883 (C) \#* $^{*}$

Viridothelium Lücking et al. (C) ${ }^{\# * *}$

Tubeufiales Boonmee \& K.D. Hyde

Tubeufiaceae M.E. Barr

Acanthohelicospora Boonmee \& K.D. Hyde $2014(\mathbf{H})^{\# *}$

Aquaphila Goh et al. $1998(\mathbf{H})^{\# *}$

Artocarpomyces Subram. 1996 (H)

Berkleasmium Zobel $1854(\mathbf{H})^{\# *}$

Camporesiomyces D.P. Wei et al. $2020(\mathbf{H})^{\# *}$

Chlamydotubeufia Boonmee \& K.D. Hyde $2011(\mathbf{H})^{\# *}$

Dematiohelicoma Y.Z. Lu et al. 2018 (H)

Dematiohelicomyces Y.Z. Lu et al. 2018 (H)

Dematiohelicosporum Y.Z. Lu et al. 2018 (H)

Dictyospora Brahamanage et al. 2017 (H) ${ }^{\# *}$ 
Helicangiospora Boonmee et al. $2014(\mathbf{H})^{\# *}$

Helicoarctatus Y.Z. Lu et al. 2018 (H)

Helicodochium J.S. Monteiro et al. 2014 (H)

Helicohyalinum Y.Z. Lu et al. 2018 (H)

Helicoma Corda $1837(\mathbf{H})^{\# *}$

Helicomyces Link $1809(\mathbf{H})^{\# *}$

Helicosporium Nees $1816(\mathbf{H})^{\# *}$

Helicotruncatum Y.Z. Lu et al. 2018 (H)

Helicotubeufia Y.Z. Lu \& J.K. Liu 2018 (H) ${ }^{\# *}$

Kamalomyces R.K. Verma et al. $2008(\mathbf{H})^{\# *}$

Kevinhydea N.G. Liu et al. 2019 (H)

Manoharachariella Bagyan. et al. 2009 (H)

Muripulchra Z.L. Luo et al. 2017 (H)

Neoacanthostigma Boonmee et al. 2014 (H) ${ }^{\# *}$

Neochlamydotubeufia Y.Z. Lu et al. 2018 (H) ${ }^{\# *}$

Neohelicomyces Z.L. Luo et al. 2017 (H)

Neohelicosporium Y.Z. Lu et al. 2017 (H)

Pleurohelicosporium Y.Z. Lu et al. 2018 (H)

Podonectria Petch $1921(\mathbf{H})^{\# *}$

Pseudohelicomyces Y.Z. Lu et al. 2018 (H)

Pseudohelicoon Y.Z. Lu \& K.D. Hyde 2018 (H)

Tamhinispora K.C. Rajeshk. \& Rahul Sharma 2013 (H)

Thaxteriellopsis Sivan. 1977 (H) ${ }^{\# *}$

Tubeufia Penz. \& Sacc. 1898 (H) ${ }^{\# *}$

Wiesneriomycetaceae Suetrong et al.

Parawiesneriomyces Crous \& M.J. Wingf. 2016 (H)

Phalangispora Nawawi \& J. Webster 1982 (H)

Pseudogliophragma Phadke \& V.G. Rao 1980 (H)

Setosynnema D.E. Shaw \& B. Sutton 1985 (H)

Speiropsis Tubaki 1958 (H)

Wiesneriomyces Koord. 1907 (H)

Valsariales Jaklitsch et al.

Valsariaceae Jaklitsch et al.

Bambusaria Jaklitsch et al. 2015 (C) (** $^{*}$

Valsaria Ces. \& De Not. 1863 (C or H) ${ }^{\# * *}$

Venturiales Yin. Zhang et al.

Sympoventuriaceae Yin. Zhang et al.

Acroconidiellina (Berk. \& Broome) M.B. Ellis 1971 (H)

Bellamyces Crous et al. 2020 (H)

Fuscohilum Crous et al. 2020 (H)

Fusicladium Bonord. 1851 (H)

Matsushimaea Subram. 1978 (H)

Mycosisymbrium Carris 1994 (H)

Neofusicladium Crous et al. 2020 (H)

Ochroconis de Hoog \& Arx $1974(\mathbf{H})^{\# *}$

Parafusicladium Crous et al. 2020 (H)

Pinaceicola Crous et al. 2020 (H)

Sympoventuria Crous \& Seifert $2007(\mathbf{H})^{\# *}$

Sterila Crous et al. 2020 (H) 
Veronaeopsis Arzanlou \& Crous 2007 (H)

Verruconis Samerp. et al. 2014 (H)

Yunnanomyces Tibpromma \& K.D. Hyde 2018 (H)

Venturiaceae E. Müll. \& Arx ex M.E. Barr

Apiosporina Höhn. 1910 with cladosporium-like asexual morph $(\mathbf{H})^{\# *}$

Caproventuria U. Braun 1998 (H) ${ }^{\# *}$

Fagicola Crous et al. 2020 (H)

Fraxinicola Crous et al. 2020 (C)

Magnohelicospora R.F. Castañeda et al. 2013 (H)

Protoventuria Berl. \& Sacc. $1887(\mathbf{H})^{\# *}$

Pseudoanungitea Crous 2018 (H)

Venturiales genera incertae sedis

Cylindrosympodioides Crous \& M.J. Wingf. 2016 (H)

Cylindrosympodium W.B. Kendr. \& R.F. Castañeda 1990 (H)

Zeloasperisporiales Hongsanan \& K.D. Hyde

Zeloasperisporiaceae Crous

Zeloasperisporium R.F. Castañeda $1996\left(\right.$ H) ${ }^{\# *}$

Dothideomycetes families incertae sedis

Alinaceae Boonmee \& K.D. Hyde

Alina Racib 1909 (H) ${ }^{\# *}$

Ascoporiaceae Kutorga \& D. Hawksw.

Pseudosolidum Lloyd 1923 with plectophomella-like asexual morph $(\mathbf{H})^{\# *}$

Balladynaceae Boonmee \& K.D. Hyde Balladyna Racib. 1900 (H) ${ }^{\# *}$

Cleistosphaeraceae Boonmee \& K.D. Hyde

Cleistosphaera Syd. \& P. Syd. 1916 (C) ${ }^{\# *}$

Dimeriaceae E. Müll. \& Arx ex Arx \& E. Müll.

Dimerium (Sacc. \& P. Syd.) McAlpine $1903(\mathbf{H})^{\# *}$

Dubujianaceae D. Pem et al.

Dubujiana D.R. Reynolds \& G.S. Gilbert 2005 (C)

Dysrhynchisceae Boonmee \& K.D. Hyde

Dysrhynchis Clem. $(\mathbf{H})^{\# *}$

Endosporiaceae D. Pem

Endosporium Tsuneda 2008 (H)

Englerulaceae Henn.

Sarcinella Sacc. $1880(\mathbf{H})^{\# *}$

Homortomycetaceae Thambug. et al.

Homortomyces Crous \& M.J. Wingf. 2012 (C) ${ }^{\# *}$ 
Leptopeltidaceae Höhn. ex Trotter

Dothiopeltis E. Müll. 1957 with idriella-like asexual morph $(\mathbf{H})^{\# * *}$

Leptopeltis Höhn. 1917 with leptothyrium-like asexual morph $(\mathbf{C})^{\# *}$

Naetrocymbaceae Höhn.

Leptorhaphis Körb. 1855 (H) ${ }^{\# *}$

Neoparodiaceae Boonmee \& K.D. Hyde

Neoparodia Petr. \& Cif. 1932 (H) (* $^{*}$

Paranectriellaceae S. Boonmee \& K.D. Hyde

Paranectriella (Henn. ex Sacc. \& D. Sacc.) Höhn. 1910 (H) ${ }^{\# *}$

Puttemansia Henn. 1902 (H) ${ }^{\# *}$

Parodiellaceae Theiss. \& Syd. ex M.E. Barr

Parodiella Speg. $1880(\mathbf{C})^{\# *}$

Perisporiopsidaceae E. Müll. \& Arx ex R. Kirschner \& T.A. Hofm.

Perisporiopsis Henn. $1904(\mathbf{H})^{\# *}$

Phaeodimeriellaceae Boonmee et al.

Phaeodimeriella Speg. 1908 (C) \#* $^{\text {H }}$

Polystomellaceae Theiss. \& P. Syd.

Dothidella Speg. 1880 (C) (** $^{\#}$

Pseudoperisporiaceae Toro

Lasiostemma Theiss. et al. 1917 (C) ${ }^{\# *}$

Pseudorobillardaceae Crous

Pseudorobillarda M. Morelet 1968 (C)

Thyrinulaceae X.Y. Zeng et al.

Blastacervulus H.J. Swart 1988 (C)

Paraopeba V.P. Abreu et al. (H)

Thyrinula Petr. \& Syd. 1924 (C)

Vizellaceae H.J. Swart

Blasdalea Sacc. \& P. Syd. $1902(\text { C) })^{\# *}$

Vizella Sacc. $1883(\text { C) })^{\# *}$

Dothideomycetes genera incertae sedis

Acanthorus Bat. \& Cavalc. 1967 (C)

Ampullifera Deighton 1960 (H)

Anguillosporella U. Braun 1995 (H)

Asterodothis Theiss. 1912 (C) \#* $^{*}$

Asteromella Pass. \& Thüm. 1880 (C)

Bahusakala Subram. 1958 (H)

Botryohypoxylon Samuels \& J.D. Rogers 1986 (C) \#* $^{*}$

Brachyconidiella R.F. Castañeda \& W.B. Kendr. 1990 (H)

Brooksia Hansf. $1956(\mathbf{H})^{\# *}$

Ceratophoma Höhn. 1917 (C) 
Chuppia Deighton 1965 (H)

Clavariopsis De Wild. 1895 (H)

Coniosporium Link 1809 (H)

Cryomyces Selbmann et al. 2005 (H)

Dactuliophora C.L. Leakey 1964 (H)

Dothichiza Lib. ex Roum. 1880 (C)

Eriomycopsis Speg. 1910 (H)

Eriothyrium Speg. 1888 (C)

Eupelte Syd. 1924 (H) ${ }^{\# *}$

Excipulariopsis P.M. Kirk \& Spooner $1982(\mathbf{H})^{\# *}$

Fusicladiella Höhn. 1919 (H)

Hansfordiella S. Hughes 1951 (H)

Hansfordiellopsis Deighton 1960 (H)

Heptaster Cif. et al. 1956 (H)

Heteroconium Petr. 1949 (H)

Kabatia Bubák 1904 (C)

Krishnamyces Hosag. 2003 (C)

Lignosphaeria Boonmee et al. 2015 (C) ${ }^{\# *}$

Marquesius L.B. Conç. et al. 2018 (H)

Monodictys S. Hughes 1958 (H)

Neodactylaria Guevara-Suarez et al. 2017 (H)

Neoovularia U. Braun 1992 (H)

Neoramularia U. Braun 1991 (H)

Neosporidesmium Mercado \& J. Mena 1988 (H)

Neottiosporina Subram. 1961 (C)

Oncopodiella G. Arnaud ex Rifai 1965 (H)

Ophiotrichum Kunze 1849 (H)

Peltaster Syd. \& P. Syd. 1917 (C)

Peltasterella Bat. \& H. Maia 1959 (C)

Pendulispora M.B. Ellis 1961 (H)

Phaeosclera Sigler et al. 1981 (H)

Placosphaeria (De Not.) Sacc. 1880 (C)

Proliferosphaera T.P. Devi 2012 (C)

Pseudoarthrographis Crous \& Thangavel 2018 (H)

Radulidium Arzanlou et al. 2007 (H)

Rupestriomyces Lei Su et al. 2015 (H)

Saxomyces L. Selbmann \& D. Isola 2014 (H)

Scleroconidioma Tsuneda et al. 2000 (H)

Scolecoxyphium Cif. \& Bat. 1956 (C)

Solicorynespora R.F. Castañeda \& W.B. Kendr. 1990 (H)

Soloacrosporiella Crous \& M.J. Wingf. 2015 (H)

Spissiomyces Lei Su et al. 2015 (H)

Trichometasphaeria Munk $1953(\mathbf{C})^{\# *}$

Troposporella P. Karst. 1892 (H)

Xenosporium Penz. \& Sacc. 1901 (H)

Class Eurotiomycetes Tehler ex O.E. Eriksson \& K. Winka

Subclass Chaetothyriomycetidae Doweld

Chaetothyriales M.E. Barr

Chaetothyriaceae Hansf. ex M.E. Barr

Aithaloderma Syd. et al. $1913(\mathbf{H})^{\# *}$

Anthracina L. Su et al. 2020 (H) 
Aphanophora Réblová et al. 2013 (H)

Arthrophiala (D.J. Soares et al.) W.S. Lisboa et al. 2016 (H)

Camptophora Réblová \& Unter. 2013 (H) ${ }^{\# *}$

Ceramothyrium Bat. \& H. Maia 1956 (=Stanhughesia Constant.) (H) ${ }^{\# *}$

Chaetothyrium Speg. $1888(\mathbf{C})^{\# * *}$

Cyphellophoriella Crous \& A. J. Smith 2015 (H)

Nullicamyces Crous 2018 (H)

Stanhughesia Constant 1989 (H)

Vonarxia Bat. 1960 (H)

Coccodiniaceae Höhn. ex O.E. Erikss.

Coccodinium A. Massal $1860(\mathbf{H})^{\# *}$

Microxiphium (Harv. ex Berk. \& Desm.) Thüm. 1879 (H) ${ }^{\# *}$

Cyphellophoraceae Réblová \& Unter.

Anthopsis Fil. March. et al. 1977 (H)

Cyphellophora G.A. de Vries 1962 (H) ${ }^{\# *}$

\section{Herpotrichiellaceae Munk}

Aculeata W. Dong et al. 2018 (H)

Brycekendrickomyces Crous \& M.J. Wingf. 2009 (H)

Cladophialophora Borelli 1980 (H)

Exophiala J.W. Carmich. 1966 (H)

Fonsecaea Negroni 1936 (H)

Marinophialophora J.F. Li et al. 2018 (H)

Melanoctona Qing Tian et al. 2016 (H)

Metulocladosporiella Crous et al. 2006 (H)

Minimelanolocus R.F. Castañeda et al. 2001 (H)

Neosorocybe Crous \& Akulov 2020 (H)

Phialophora Medlar 1915 (H)

Nadsoniella Issatsch. 1914 (H)

Phialophora Medlar 1915 (H)

Rhinocladiella Nannf. 1934 (H)

Sorocybe Fr. 1849 (H)

Thysanorea Arzanlou et al. 2007 (H)

Veronaea Cif. \& Montemart. 1957 (H)

\section{Lyrommataceae Lücking}

Lyromma Bat. \& H. Maia 1965 (C)

Paracladophialophoraceae Crous

Paracladophialophora Crous 2016 (H)

Pyrenotrichaceae Zahlbr

Neophaeococcomyces Crous et al. 2015 (H)

Strelitzianaceae Crous \& M.J. Wingf.

Neophaeococcomyces Crous et al. 2015 (H)

Strelitziana Arzanlou et al. 2006 (H)

Trichomeriaceae Chomnunti \& K.D. Hyde Arthrocladium Papendorf 1969 (H) 
Bradymyces Hubka et al. 2014 (H)

Knufia L.J. Hutchison \& Unter. 1996 (H)

Lithohypha Selbmann \& Isola 2017 (H)

Lithophila Selbmann et al. 2019 (H)

Neostrelitziana Crous et al. 2015 (H)

Strelitziana Arzanlou et al. 2006 (H)

Trichomerium Speg. 1918 (H) ${ }^{\# *}$

Chaetothyriales genera incertae sedis

Atrokylindriopsis Y.R. Ma \& X.G. Zhang 2015 (H)

Bacillicladium Hubka et al. 2016 (H)

Lichenodiplis Dyko \& D. Hawksw. 1979 (C)

Lichenodiplisiella S.Y. Kondr. \& Kudratov 2002 (C)

Melnikomyces Crous \& U. Braun 2014 (H)

Minutoexcipula V. Atienza \& D. Hawksw. 1994 (H)

Muellerella Hepp et al. $1862(\mathbf{C})^{\# *}$

Sarcinomyces Lindner 1898 (H)

Uncispora R.C. Sinclair et al. 1979 (H)

Phaeomoniellales K.H. Chen et al.

Celotheliaceae Lücking et al. (= Phaeomoniellaceae P.M. Kirk)

Aequabiliella Crous 2015 (C)

Celerioriella Crous 2015 (H)

Minutiella Crous 2015 (H)

Neophaeomoniella Rooney-Latham \& Crous 2015 (C)

Paraphaeomoniella Crous 2015 (C)

Phaeomoniella Crous \& W. Gams 2000 (H)

Pseudophaeomoniella Nigro et al. 2015 (C)

Xenocylindrosporium Crous \& Verkley 2009 (C)

Phaeomoniellales genus incertae sedis

Vredendaliella C.F.J. Spies et al. 2020 (C)

Pyrenulales Fink ex D. Hawksw. \& O.E. Erikss.

Verrucariaceae Zenker

Phaeospora Hepp et al. 1867 (H)

Verrucariales genera incertae sedis

Botryolepraria Canals et al. 1997 (H)

Chaetothyriomycetidae family incertae sedis

Rhynchostomataceae Winka \& O.E. Erikss.

Dolabra C. Booth et al.1964 with rhabdospora-like asexual morph $(\mathbf{C})^{\# *}$

Subclass Eurotiomycetidae Geiser \& Lutzoni

Arachnomycetales Gibas et al.

Arachnomycetaceae Gibas et al.

Arachnomyces Massee et al. 1902 (H)

Onychocola Sigler 1990 (H)

Eurotiales G.W. Martin ex Benny \& Kimbr.

Aspergillaceae Link 
Aspergillus P. Micheli ex Link $1809(\mathbf{H})^{\# *}$

Dichlaena Durieu et al.1848 (H) ${ }^{\# *}$

Hamigera Stolk \& Samson $1971(\mathbf{H})^{\# *}$

Monascus Tiegh. 1884 (H) ${ }^{\# *}$

Penicillago Guevara-Suarez et al. 2020 (H)

Penicilliopsis Solms et al. $1887(\mathbf{H})^{\# *}$

Penicillium Link 1809 (H) ${ }^{\# *}$

Phialomyces P.C. Misra \& P.H.B. Talbot 1964 (H)

Pseudopenicillium Guevara-Suarez et al. 2020 (H)

Pseudohamigera Houbraken et al. 2020 (H)

Sclerocleista Subram. $1972(\mathbf{H})^{\# *}$

Xerochrysium Pitt 2013 (H)

Xeromyces Fraser et al. $1953(\mathbf{H})^{\# *}$

Thermoascaceae Apinis

Paecilomyces Bainier 1907 (H) ${ }^{\# * *}$

Thermoascus Miehe 1907 (H) ${ }^{\# *}$

Trichocomaceae E. Fisch.

Acidotalaromyces Houbraken et al. 2020 (H)

Ascospirella Houbraken et al. 2020 (H)

Evansstolkia Houbraken et al. 2020 (H)

Rasamsonia Houbraken et al. 2011 (H) ${ }^{\# *}$

Sagenomella W. Gams 1978 (H)

Talaromyces C.R. Benj. 1955 (H) ${ }^{\# *}$

Thermomyces Tsikl. 1899 (H)

Onygenales Cif. ex Benny \& Kimbr.

Ajellomycetaceae Untereiner et al.

Blastomyces Gilchrist \& W.R. Stokes 1898 (H)

Emergomyces Dukik et al. 2017 (Y)

Emmonsiellopsis Y. Marín et al. 2015 (H)

Histoplasma Darling 1906 (H)

Lacazia Taborda et al. 1999 (H)

Paracoccidioides F.P. Almeida 1930 (H)

Arthrodermataceae Currah

Epidermophyton Sabour. 1907 (H)

Guarromyces Y Gräser \& de Hoog 2018 (H)

Microsporum Gruby 1843 (H)

Nannizzia Stockdale 1961 (H)

Paraphyton Gräser et al. 2018 (H)

Trichophyton Malmsten 1848 (H)

Ascosphaeraceae L.S. Olive \& Spiltoir

Ascosphaera L.S. Olive et al. 1955 with chrysosporium-like asexual morph $(\mathbf{H})^{\# *}$

Bettsia Skou 1972 with chrysosporium-like asexual morph $(\mathbf{H})^{\# *}$

\section{Gymnoascaceae Baran.}

Gymnascella Peck $1884(\mathbf{H})^{\# *}$

Oncocladium Wallr. 1833 (H)

Orromyces B. Sur et al. 1987 with chrysosporium-like asexual morph (H) ${ }^{\# *}$ 
Nannizziopsidaceae Guarro et al.

Nannizziopsis Currah 1985 (H) ${ }^{\# *}$

Onygenaceae Berk.

Auxarthronopsis Rah. Sharma et al. $2013(\mathbf{H})^{\# *}$

Canomyces Rahul Sharma \& Shouche 2020 (C)

Chrysosporium Corda 1833 (H)

Coccidioides G.W. Stiles 1896 (H)

Malbranchea Sacc. 1882 (H)

Myriodontium Samson \& Polon. 1978 (H)

Neogymnomyces G.F. Orr $1970(\mathbf{H})^{\# *}$

Ophidiomyces Sigler et al. 2013 (H)

Paranannizziopsis Sigler et al. 2013 (H)

Pectinotrichum Varsavsky et al. 1971 (H)

Sporendonema Desm. 1827 (H)

Spiromastigaceae Hirooka et al.

Pseudospiromastix Guarro et al. $2016(\mathbf{H})^{\# *}$

Sigleria Hirooka et al. 2016 (H)

Spiromastigoides Doweld 2013 (= Spiromastix Kuehn \& G.F. Orr) $(\mathbf{H})^{\# *}$

Onygenales genera incertae sedis

Arthropsis Sigler et al. 1982 (H)

Ovadendron Sigler et al. 1976 (H)

Sphaerosporium Schwein. 1832 (H)

Subclass Mycocaliciomycetidae Tibell

Mycocaliciales Tibell \& Wedin

Mycocaliciaceae A.F.W. Schmid

Brunneocarpos Giraldo \& Crous $2016(\mathbf{H})^{\# *}$

Chaenothecopsis Vain. $1927(\mathbf{H})^{\# *}$

Mycocalicium Vain. ex Reinke 1890 (H) ${ }^{\# *}$

Sclerococcomycetidae Réblová et al.

Sclerococcales Réblová et al.

Dactylosporaceae Bellem. \& Hafellner (= Sclerococcaceae Réblová et al.)

Cylindroconidiis Zhang et al. 2018(H)

Fusichalara Hughes et al. 1973 (H)

Gamsomyces Hern.-Restr. \& Réblová 2020 (H)

Pseudobactrodesmium H. Zhang et al. 2020 (H)

Rhopalophora Réblová et al. 2016 (H)

Sclerococcum Fr. 1825 (= Dactylospora Körb.) (H)

Eurotiomycetes genera incertae sedis

Cirrosporium S. Hughes 1980 (C)

Neocladophialophora Crous et al. 2014 (H)

Class Laboulbeniomycetes Engler

Laboulbeniales genus incertae sedis

Coreomycetopsis Thaxt. 1920 (H)

Pyxidiophorales P.F. Cannon 
Pyxidiophoraceae Arnold

Gliocephalis Matr. 1899 (H)

Pleurocatena G. Arnaud ex Aramb. et al. 2007 (H)

Class Lecanoromycetes O.E. Erikss. \& Winka

Subclass Ostropomycetidae Reeb et al.

Ostropales Nannf.

Stictidaceae Fr.

Acarosporina Sherwood 1977 with phacidiella-like asexual morph $(\mathbf{C})^{\# *}$

Cyanodermella O.E. Erikss. 1981 (C)

Fitzroyomyces Crous 2017 (H)

Glomerobolus Kohlm. \& Volkm.-Kohlm. 1996 (H)

Neofitzroyomyces Crous 2018 (H)

Stictis Pers. 1800 (= Stictospora Cif.) (C) ${ }^{\# *}$

Stictophacidium Rehm 1888 with coleophoma-like asexual morph $(\mathbf{C})^{\# *}$

Subclass Lecanoromycetidae P.M. Kirk et al.

Lecanorales Nannf.

Stereocaulaceae Chevall.

Lepraria Ach. 1803 (C)

Lecanorales genus incertae sedis

Lichenosticta Zopf 1898 (C)

Leprocaulales Lendemer \& B.P. Hodk.

Leprocaulaceae Lendemer \& B.P. Hodk.

Leprocaulon Nyl. 1878 (C)

Ostropales Nannf.

Graphidaceae Dumort.

Rubikia H.C. Evans \& Minter 1985 (C)

Ostropales genera incertae sedis

Elongaticonidia W.J. Li et al. 2020 (C)

Mulderomyces Crous et al. 2016 (C)

Peltigerales Walt. Watson

Collemataceae Zenker

Paracollema Otálora \& Wedin $2014(\text { C) })^{\# *}$

Lecanoromycetes order incertae sedis

Micropeltidales X.Y. Zeng, H.X. Wu \& K.D. Hyde

Micropeltidaceae Clem. \& Shear

Cyclopeltella Petr. 1953 (C) ${ }^{\# *}$

Stomiopeltis Theiss. 1914 (as Akaropeltella M.L. Farr 1972) with sporidesmium-like asexual $\operatorname{morph}(\mathbf{H})^{\# *}$

Class Leotiomycetes O.E. Erikss. \& Winka

Chaetomellales Crous \& Denman

Chaetomellaceae Baral et al.

Chaetomella Fuckel $1870(\mathbf{C})^{\# *}$

Pilidium Kunze 1823 (C) (** $^{\# *}$ 
Sphaerographium Sacc. 1884 (C)

Synchaetomella Decock \& Seifert 2005 (H)

Helotiales Nannf. (= Erysiphales Gwynne-Vaughan)

Amorphothecaceae Parbery

Amorphotheca Parbery 1969 (H) \#* $^{\# *}$

Ascodichaenaceae D. Hawksw. \& Sherwood

Ascodichaena Butin 1977 (C) \#*

Bloxamiaceae Locq.

Bloxamia Berk. \& Broome $1854(\mathbf{H})^{\# *}$

Calloriaceae Marchand

Calloria Fr. $1836(\mathbf{H})^{\# *}$

Cenangiaceae Rehm

Crumenulopsis J.W. Groves 1969 (C) ${ }^{\# *}$

Mycosphaerangium Verkley 1999 (C) ${ }^{\# * *}$

Neomelanconium Petr. 1940 (C) ${ }^{\# * *}$

Rhabdocline Syd. $1922(\mathbf{H})^{\# *}$

Chlorociboriaceae Baral \& P.R. Johnst.

Chlorociboria Seaver ex Ramamurthi et al. 1958 (C) $)^{\# *}$

Cordieritidaceae Sacc.

Diplolaeviopsis Giralt \& D. Hawksw. 1991 (C)

Sabahriopsis Crous \& M.J. Wingf. 2015 (H)

Unguiculariopsis Rehm 1909 (C) (** $^{*}$

Dermateaceae Fr.

Coleophoma Höhn. 1907 (C)

Corniculariella P. Karst. 1884 (C)

Dermea Fr. 1825 (C) \#* $^{*}$

Neodermea W.J. Li et al. 2020 (C)

Neofabraea H.S. Jacks. 1913 (C) \#*

Neogloeosporidina W.J. Li et al. 2020 (C)

Pezicula Tul. \& C. Tul. 1865 (C) ${ }^{\# *}$

Phlyctema Desm. 1847 (C)

Pseudofabraea Chen et al. 2016 (C)

Verkleyomyces Y. Marín \& Crous 2017 (C) \#* $^{\text {* }}$

Xenochalara M.J. Wingf. \& Crous 2000 (H)

Discinellaceae Ekanayaka \& K.D. Hyde

Articulospora Ingold 1942 (H)

Cladochasiella Marvanová 1997 (H)

Fontanospora Dyko 1978 (H)

Gyoerffyella Kol 1928 (H)

Lemonniera De Wild. 1894 (H)

Margaritispora Ingold 1942 (H)

Pseudopezicula Korf 1986 with phialophora-like asexual morph (H) $)^{\# *}$

Tetrachaetum Ingold 1942 (H) 
Varicosporium W. Kegel 1906 (H)

Drepanopezizaceae Baral

Blumeriella Arx 1961 (C) $)^{\# *}$

Diplocarpon F.A. Wolf 1912 (C) or (H) ${ }^{\# *}$

Leptotrochila P. Karst. 1871 (C) $)^{\# *}$

Erysiphaceae Tul. \& C. Tul.

Blumeria Golovin ex Speer 1975 (H) ${ }^{\# *}$

Bulbomicroidium Marm. et al. 2017 (H)

Erysiphe R. Hedw. ex DC. 1805 (H) ${ }^{\# *}$

Leveillula $\mathrm{G}$. Arnaud 1921 (H) ${ }^{\# *}$

Neoerysiphe U. Braun 1999 (H) ${ }^{\# *}$

Microidium (To-anun \& S. Takam.) To-anun \& S. Takam. 2012 (H)

Phyllactinia Lév. 1851 (H) ${ }^{\# *}$

Pleochaeta Sacc. \& Speg. 1881 (H) ${ }^{\# *}$

Podosphaera Kunze $1823(\mathbf{H})^{\# *}$

Sawadaea Miyabe $1914(\mathbf{H})^{\# *}$

\section{Gelatinodiscaceae S.E.Carp}

Ascocoryne J.W. Groves \& D.E. Wilson 1967 (H) ${ }^{\# *}$

Dimorphospora Tubaki 1958 (H)

Neobulgaria Petr. 1921 with myrioconium-like or phialophora-like asexual morph $(\mathbf{H})^{\# *}$

Xerombrophila Baral $2013(\mathbf{H})^{\# *}$

\section{Godroniaceae Baral}

Ascocalyx Naumov $1926(\mathbf{C})^{\# *}$

Godronia Moug. \& Lév. 1846 (C) $)^{\# *}$

Gremmeniella M. Morelet $1969(\mathbf{C})^{\# *}$

\section{Helotiaceae Rehm}

Ascoconidium Seaver $1942(\mathbf{H})^{\# *}$

Filosporella Nawawi 1976 (H)

Geniculospora Sv. Nilsson ex Marvanová \& Sv. Nilsson 1971 (H)

Glarea Bills \& Paláez 1999 (H)

Gloeotinia M. Wilson et al. 1954 (H) ${ }^{\# *}$

Helicodendron Peyronel 1918 (H)

Hymenoscyphus Gray 1821 (H) (* $^{*}$

Mycofalcella Marvanová et al. 1993 (H)

Neocrinula Crous 2016 (H)

Scytalidium Pesante 1957 (H) ${ }^{\# *}$

Symphyosirinia E.A. Ellis $1956(\mathbf{H})^{\# *}$

Tricladium Ingold 1942 (H)

Heterosphaeriaceae Rehm

Heterosphaeria Grev. 1824 (C) $)^{\# *}$

Hyaloscyphaceae Nannf.

Arbusculina Marvanová \& Descals 1987 (H)

Cistella Quél. 1886 (H) ${ }^{\# *}$

Clathrosphaerina Beverw. 1951 (H)

Crucellisporiopsis Nag Raj 1983 (C) 
Dematioscypha Svrček $1977(\mathbf{H})^{\# *}$

Hyalopeziza Fuckel 1870 (H) ${ }^{\# *}$

Hyaloscypha Boud. $1885(\mathbf{H})^{\# *}$

Hyphodiscus Kirschst. 1906 (H) ${ }^{\# *}$

Mycoarthris Marvanová \& P.J. Fisher 2002 (H)

Leptodontidiaceae Hern.-Restr. et al.

Leptodontidium de Hoog. 1979 (H)

Mollisiaceae Rehm

Barrenia E. Walsh \& N. Zhang 2015 (H)

Cheirospora Moug. \& Fr. 1825 (C)

Cystodendron Bubák 1914 (H)

Fuscosclera Hern.-Restr. et al. 2017 (H)

Mollisia (Fr.) P. Karst. 1871 (H) ${ }^{\# *}$

Phialocephala W.B. Kendr. 1961 (H) ${ }^{\# *}$

Trimmatostroma Corda 1837 (H)

Variocladium Descals \& Marvanová (H)

Myxotrichaceae Currah

Oidiodendron Robak 1932 (H)

\section{Neolauriomycetaceae Crous}

Exochalara W. Gams \& Hol.-Jech. 1976 (H)

Lareunionomyces Crous \& M.J. Wingf. 2016 (H)

Neolauriomyces Crous 2018 (H)

Pezizellaceae Velen.

Calycellina Höhn $1918(\mathbf{H})^{\# *}$

Chalara (Corda) Rabenh. 1844 (H)

Ciliolarina Svrcek 1977 (H) ${ }^{\# *}$

Scleropezicula Verkley 1999 (C) ${ }^{\# *}$

Curviclavula G. Delgado et al. 2015 (H)

Hyalodendriella Crous 2007 (H)

Xenopolyscytalum Crous 2010 (H)

Zymochalara Guatim. et al. 2016 (H)

\section{Ploettnerulaceae Kirschst.}

Cadophora Lagerb. \& Melin (H)

Collembolispora Marvanová \& Pascoal 2003 (H)

Mastigosporium Riess 1852 (H)

Mycochaetophora Hara \& Ogawa 1931 (H)

Oculimacula Crous \& W. Gams $2003(\mathbf{H})^{\# *}$

Pyrenopeziza Fuckel $1870(\mathbf{C})^{\# *}$

Rhynchosporium Heinsen ex A.B. Frank 1897 (H)

Ypsilina J. Webster et al. 1999 (H)

\section{Rutstroemiaceae Holst-Jensen et al.}

Neometulocladosporiella Crous \& M.J. Wingf. 2018 (H)

Rutstroemia P. Karst. 1871 with phialophora-like asexual morph $(\mathbf{H})^{\# *}$

Sclerotiniaceae Whetzel ex Whetzel 
Amphobotrys Hennebert 1973 (H)

Botrytis P. Micheli ex Pers. $1794(\mathbf{H})^{\# *}$

Cristulariella Höhn. $1916(\mathbf{H})^{\# *}$

Grovesinia M.N. Cline et al. 1983 (H) ${ }^{\# *}$

Haradamyces Masuya et al. 2009 (H)

Monilinia Honey 1928 (H) ${ }^{\# *}$

Mycopappus Redhead \& G.P. White 1985 (H)

Myrioconium Syd. \& P. Syd. 1912 (H)

Ovulinia F.A. Weiss 1940 (H) ${ }^{\# *}$

Pycnopeziza W.L. White \& Whetzel 1938 (C) \#* $^{\# *}$

Sclerotium Tode 1790 (H)

Seaverinia Whetzel 1945 (H) ${ }^{\# *}$

Septotinia Whetzel ex J.W. Groves \& M.E. Elliott 1961 (H) ${ }^{\# *}$

Streptotinia Whetzel $1945(\mathbf{H})^{\# *}$

Valdensia Peyronel $1923(\mathbf{H})^{\# *}$

\section{Vibrisseaceae Korf}

Acephala Grünig \& T.N. Sieber 2005 (H)

Chlorovibrissea L.M. Kohn 1989 with phialophora-like asexual morph $(\mathbf{H})^{\# *}$

Srinivasanomyces S. Rana \& S.K. Singh 2020 (H)

Vibrissea Fr. $1822(\mathbf{H})^{\# *}$

Helotiales genera incertae sedis

Acidea Hujslová \& M. Kolařík 2014 (H)

Acidomelania E. Walsh \& N. Zhang 2014 (H)

Aphanodesmium Réblová \& Hern.-Restr. 2020 (C)

Apiculospora Wijayaw. et al. 2016 (C)

Bioscypha Syd. 1927 with rhinocladiella-like asexual morph $(\mathbf{H})^{\# *}$

Brachyalara Réblová \& W. Gams 2011 (H)

Brefeldochium Verkley 2005 (H)

Clathrosporium Nawawi \& Kuthub. 1987 (H)

Crucellisporium M.L. Farr 1968 (C)

Dactylaria Sacc. 1880 (H)

Fulvoflamma Crous 2006 (H)

Godroniopsis Diehl \& E.K. Cash 1929 (C) \#*

Humicolopsis Cabral \& S. Marchand 1976 (H)

Infundichalara Réblová \& W. Gams 2011 (H)

Lareunionomyces Crous \& M.J. Wingf. 2016 (H)

Libartania Nag Raj 1979 (C)

Monochaetiellopsis B. Sutton \& DiCosmo 1977 (C) $)^{\# *}$

Phacidiella P. Karst. 1884 (C)

Rhexocercosporidium U. Braun 1994 (H)

Rhizocladosporium Crous \& U. Braun 2007 (H)

Rhizothyrium Naumov 1915 (C) ${ }^{\# *}$

Soosiella Hujslová \& M. Kolařík 2014 (H)

Spirosphaera Beverw. 1953 (H)

Stamnaria Fuckel $1870(\mathbf{C})^{\# *}$

Strossmayeria Schulzer $1881(\mathbf{H})^{\# *}$

Tetracladium De Wild. $1893(\mathbf{H})$

Thedgonia B. Sutton 1973 (H)

Triposporium Corda 1837 (H)

Vandijckella Sandoval-Denis 2017 (H) 
Lauriomycetales Hern.-Restr. et al.

Lauriomycetaceae Hern.-Restr. et al.

Lauriomyces R.F. Castañeda 1990 (H)

Leotiales Carpenter

Cochlearomycetaceae Crous

Cochlearomyces Crous 2017 (C)

Satchmopsis B. Sutton \& Hodges 1975

\section{Leotiaceae Corda}

Halenospora E.B.G. Jones 2009 (H)

Miniancora Marvanová \& Bärl. 1989 (H)

Tympanidaceae Baral \& Quijada

Claussenomyces Kirschst. 1923 (H) ${ }^{\# *}$

Collophorina Damm \& Crous 2017 (C)

Gelatinosporium Peck 1873 (C)

Pragmopora A. Massal 1855 (C) \#*

Tympanis Tode $1790(\mathbf{C})^{\# *}$

Leotiales genera incertae sedis

Alatospora Ingold 1942 (H)

Flagellospora Ingold 1942 (H)

Gelatinomyces N. Sanoamuang et al. $2013(\mathbf{H})^{\# *}$

Micraspidales Quijada \& Tanney

Micraspidaceae Quijada \& Tanney

Micraspis Darker $1963\left(\right.$ C) ${ }^{\# *}$

Phacidiales C.E. Bessey

Helicogoniaceae Baral

Eleutheromycella Höhn. 1908 (C)

Eleutheromyces Fuckel 1870 (C)

Gelatinipulvinella Hosoya \& Y. Otani 1995 (H) ${ }^{\# *}$

Phacidiaceae Fr.

Allantophomopsiella Crous 2014 (C)

Allantophomopsis Petr. 1925 (C)

Phacidiopycnis Potebnia 1912 (C) \#* $^{\# *}$

Phacidium Fr. 1815 (C) \#* $^{\text {\#* }}$

Phacidiales genera incertae sedis

Coma Nag Raj \& W.B. Kendr. 1972 (C) \#* $^{\# *}$

Rhytismatales M.E. Barr ex Minter

Rhytismataceae Chevall.

Hypohelion P.R. Johnst. 1990 (C) ${ }^{\# *}$

Ploioderma Darker. 1967 (C) ${ }^{\# *}$

Rhytisma Fr. 1818 (C) ${ }^{\# *}$

Thelebolales P.F. Cannon

Pseudeurotiaceae Malloch \& Cain

Geomyces Traaen 1914 (H) 
Gymnostellatospora Udagawa et al. 1993 (C)

Thelebolaceae (Brumm.) Eckblad

Solomyces Zhi Y. Zhang et al. 2020 (H)

Leotiomycetes families incertae sedis

Porodiplodiaceae Crous

Porodiplodia Crous 2018 (C)

Leotiomycetes genera incertae sedis

Epicladonia D. Hawksw. 1981 (C)

Gorgomyces M. Gönczöl \& Révay 1985 (H)

Helicocentralis Sri-indr. et al. 2015 (H)

Holwaya Sacc. 1889 (H) ${ }^{\# *}$

Leohumicola N.L. Nick. 2005 (H)

Ocotomyces H.C. Evans \& Minter $1985(\text { C })^{\# *}$

Trullula Ces. 1852 (C)

Class Orbiliomycetes O.E. Erikss. \& Baral

Orbiliales Baral et al.

Orbiliaceae Nannf.

Arthrobotrys Corda 1939 (H) \#* $^{\# *}$

Dactylella Grove 1884 (H)

Dactylellina M. Morelet 1968 (H)

Drechslerella Subram. 1964 (H)

Gamsylella M. Scholler et al. 1999 (H)

Helicoön Morgan 1892 (H)

Hyalorbilia Baral \& G. Marson $2001(\mathbf{H})^{\# *}$

Orbilia Fr. $1836(\mathbf{H})^{\# *}$

Pseudotripoconidium Z.F. Yu \& K.Q. Zhang 2011 (H)

Retiarius D.L. Olivier 1978 (H)

Vermispora Deighton \& Piroz. 1972 (H)

Orbiliales genus incertae sedis

Microdochiella Hern.-Restr. \& Crous 2015 (H)

Class Pezizomycetes sensu O.E. Erikss. \& Winka

Pezizales J. Schröt.

Ascobolaceae Boud. ex Sacc.

Cubonia Sacc. 1889 with papulospora-like asexual morph $(\mathbf{H})^{\# *}$

Ascodesmidaceae J. Schröt.

Cephaliophora Thaxt. 1903 (H)

Caloscyphaceae Harmaja

Caloscypha Boud. $1885(\mathbf{H})^{\# *}$

Chorioactidaceae Pfister

Desmazierella Lib. $1829(\mathbf{H})^{\# *}$

Morchellaceae Reichenb.

Morchella Dill. ex Pers.1794 (H) ${ }^{\# *}$ 
Pezizaceae Dumort. (= Carbomycetaceae Trappe)

Chromelosporiopsis Hennebert 2020 (H)

Pseudombrophilaceae Ekanayaka et al.

Heydenia Fresen. 1852 (H)

Pyronemataceae Corda

Tricharina Eckblad 1968 (H) ${ }^{\# *}$

Micronematobotrys Xiang Sun \& L.D. Guo 2010 (H)

Miladina Svrček $1972(\mathbf{H})^{\# *}$

Trichophaea Boud. $1885(\mathbf{H})^{\# *}$

Rhizinaceae Bonord.

Phymatotrichopsis Hennebert 1973 (H)

Sarcoscyphaceae LeGal ex Eckblad

Sarcoscypha (Fr.) Boud. $1885(\mathbf{H})^{\# *}$

Sarcosomataceae Kobayasi

Conoplea Pers. 1797 (H)

Strumella Fr. 1849 (H)

Class Sordariomycetes O.E. Erikss. \& Winka

Subclass Diaporthomycetidae Senan. et al.

Annulatascales D'souza et al.

Annulatascaceae S.W. Wong et al.

Annulatascus Ces. \& De Not. 1863 (C)

Chaetorostrum Zelski et al. 2011. with taeniolella-like asexual morph (H) ${ }^{\# *}$

Conlariaceae $\mathrm{H}$. Zhang et al.

Conlarium F. Liu \& L. Cai $2012(\mathbf{H})^{\# *}$

Calosphaeriales M.E. Barr

Calosphaeriaceae Munk

Calosphaeria Tul. \& C Tul.1863 (H) ${ }^{\# *}$

Flabellascus Réblová 2015 (H) ${ }^{\# *}$

Jattaea Berl 1900 (H) ${ }^{\# *}$

Togniniella Réblová et al. $2004(\mathbf{H})^{\# *}$

Pleurostomataceae Réblová et al.

Pleurostoma Tul. \& C. Tul. $1863(\mathbf{H})^{\# *}$

Diaporthales Nannf.

Apiosporopsidaceae Senan. et al.

Apiosporopsis (Traverso) Mariani. 1911 (C) \#*

Apoharknessiaceae Senan. et al.

Apoharknessia Crous \& S.J. Lee 2004 (C)

Lasmenia Speg. 1886 (C)

Asterosporiaceae Senan. et al.

Asterosporium Kunze 1819 (C) 
Auratiopycnidiellaceae Senan. et al.

Auratiopycnidiella Crous \& Summerell 2012 (C)

Coryneaceae Corda

Coryneum Nees $1817(\mathbf{C})^{\# *}$

Hyaloterminalis Rathnayaka et al. 2020 (C)

Talekpea Lunghini \& Rambelli 1979 (H)

Cryphonectriaceae Gryzenh. \& M.J. Wingf.

Amphilogia Gryzenhout et al. 2005 (C)

Aurantiosacculus Dyko \& B. Sutton 1979 (C)

Aurapex Gryzenh. \& M.J. Wingf. 2006 (C)

Aurifilum Begoude et al. 2010 (C)

Celoporthe Nakab. et al. 2006 (C)

Chrysofolia Crous \& M.J. Wingf. 2015 (C)

Chrysomorbus S.F. Chen 2017 (C)

Chrysoporthe Gryzenh. \& M.J. Wingf. 2004 (C)

Corticimorbus S.F. Chen \& M.J. Wingf. 2016 (C)

Cryphonectria (Sacc.) Sacc. \& D. Sacc. 1905 (C) \#*

Cryptometrion Gryzenh. \& M.J. Wingf. 2010 (C)

Diversimorbus S.F. Chen \& J. Roux 2014 (C)

Endothia Fr.1849 (C)

Eriocamporesia R.H. Perera et al. 2020 (C) \#* $^{*}$

Foliocryphia Cheew. \& Crous 2009 (C)

Holocryphia Gryzenh. \& M.J. Wingf. 2006 (C)

Immersiporthe S.F. Chen et al. 2013 (C)

Latruncellus M. Verm. et al. 2011 (C)

Luteocirrhus C.F. Crane \& T.I. Burgess 2013 (C)

Mastigosporella Höhn. 1914 (C)

Microthia Gryzenh. \& M.J. Wingf. 2006 (C) (\#* $^{\# *}$

Myrtonectria Marinc. et al. 2018 (C)

Neocalonectria Crous 2020 (H)

Parvomorbus W. Wang \& S.F. Chen 2020 (C)

Rostraureum Gryzenh. \& M.J. Wingf. 2005 (C)

Ursicollum Gryzenh. \& M.J. Wingf. 2006 (C)

Wuestneia Auersw. ex Fuckel 1864 (C) ${ }^{\# *}$

\section{Cytosporaceae Fr.}

Cytospora Ehrenb. 1818 (C)

Pachytrype Berl. ex M.E. Barr et al. 1993 (C)

Waydora B. Sutton 1976 (C)

Diaporthaceae Höhn. ex Wehm.

Apiosphaeria Höhn. 1909 (C) \#* $^{*}$

Chaetoconis Clem. 1909 (C)

Chiangraiomyces Senan. et al. 2017 (C) $\# *$

Diaporthe Nitschke $1870(\mathbf{C})^{\# *}$

Hyaliappendispora Senan. et al. $2017(\mathbf{C})^{\# *}$

Massariothea Syd. 1939 (C)

Mazzantia Mont. et al. 1855 (C) \#* $^{\text {* }}$

Ophiodiaporthe Y.M. Ju et al. 2013 (C) \#*

Phaeocytostroma Petr. 1921 (C)

Pustulomyces D.Q. Dai et al. 2014 (C) 
Stenocarpella Syd. \& P. Syd. 1917 (C)

Diaporthosporellaceae C.M. Tian \& Q. Yang

Diaporthosporella C.M. Tian \& Q. Yang 2017 (C) \#** $^{*}$

Dwiroopaceae K.V. Xavier et al.

Dwiroopa Subram. \& Muthumary 1986 (C)

Erythrogloeaceae Senan. et al.

Chrysocrypta Crous \& Summerell 2012 (C)

Disculoides Crous et al. 2012 (C)

Erythrogloeum Petr. 1953 (C)

Foliocryphiaceae C.M. Tian et al.

Neocryphonectria C.M. Tian et al. $2020(\text { C) })^{\# *}$

Gnomoniaceae G. Winter

Apiognomonia Höhn. 1917(C) \#*

Asteroma DC. et al. 1815 (C)

Cryptosporella Sacc. $1877(\mathbf{C})^{\# *}$

Gloeosporidina Petr. 1921 (C)

Millerburtonia Cif. 1951 (C)

Sirococcus Preuss 1855 (C)

Uleoporthe Petr. 1941 (C) $)^{\# *}$

Valsalnicola D.M. Walker \& Rossman 2012 (C) ${ }^{\# *}$

Harknessiaceae Crous

Harknessia Cooke $1881(\mathbf{C})^{\# *}$

Mebarria J. Reid \& C. Booth $1989(\text { C) })^{\# *}$

Juglanconidaceae Voglmayr \& Jaklitsch

Juglanconis Voglmayr \& Jaklitsch 2017 (C) \#* $^{*}$

Lamproconiaceae C. Norphanphoun et al.

Hercospora Fr. 1825 (C) (** $^{\text {* }}$

Lamproconium (Grove) Grove 1937 (C)

\section{Macrohilaceae Crous}

Macrohilum H.J. Swart 1988 (C)

Melanconidaceae G. Winter

Melanconis Tul. \& C. Tul. 1863 (C) (** $^{\# *}$

Melanconiellaceae Senan. et al.

Dicarpella Syd. \& P. Syd. 1921 (C) ${ }^{\# *}$

Greeneria Scribn. \& Viala 1887 (C)

Massariovalsa Sacc. $1882(\mathbf{C})^{\# *}$

Melanconiella Sacc. 1882 (C) \#* $^{\# *}$

Septomelanconiella Samarak. \& K.D. Hyde 2019 (C)

Sheathospora X.L. Fan 2018 (C)

Neomelanconiellaceae Crous 
Neomelanconiella Crous 2018 (C)

Phaeoappendicosporaceae Crous \& M.J. Wingf.

Neophaeoappendicospora Crous \& M.J. Wingf. 2019 (C) (** $^{*}$

Prosopidicolaceae Senan. \& K.D. Hyde

Prosopidicola Crous \& C.L. Lennox 2004 (C)

Pseudomelanconidaceae C.M. Tian \& X.L. Fan

Pseudomelanconis C.M. Tian \& X.L. Fan 2018 (C)

Neopseudomelanconis C.M. Tian \& N. Jiang 2018 (C)

Pseudoplagiostomataceae Cheew. et al.

Pseudoplagiostoma Cheew. et al. 2010 (C) ${ }^{\# * *}$

Schizoparmaceae Rossman

Coniella Höhn. 1918 (C) \#* $^{\text {\#* }}$

Stilbosporaceae Link

Crinitospora B. Sutton \& Alcorn 1985 (C)

Natarajania Pratibha \& Bhat 2005 (C)

Stegonsporium Corda $1827(\mathbf{C})^{\# *}$

Stilbospora Pers. $1794(\mathbf{C})^{\# *}$

Sydowiellaceae Lar.N. Vassiljeva

Hapalocystis Auersw. ex Fuckel 1863 (C) $)^{\# *}$

Lambro Racib. 1900 (C) ${ }^{\# *}$

Paragnomonia Senan. \& K.D. Hyde 2017 (C) \#* $^{\#}$

Tenuiappendicula Senan. et al. 2017 (C) ${ }^{\# *}$

Synnemasporellaceae X.L. Fan \& J.D.P. Bezerra

Synnemasporella X.L. Fan $2018(\mathbf{C} \text { or H })^{\# *}$

Tubakiaceae U. Braun et al.

Involutscutellula U. Braun \& C. Nakash. 2018 (C)

Oblongisporothyrium U. Braun \& C. Nakash. 2018 (C)

Paratubakia U. Braun \& C. Nakash. 2018 (C)

Racheliella Crous \& U. Braun 2018 (C)

Saprothyrium U. Braun et al. 2018 (C)

Sphaerosporithyrium U. Braun et al. 2018 (C)

Tubakia B. Sutton 1973 (C) \#* $^{*}$

Distoseptisporales Z.L. Luo et al.

Distoseptisporaceae K.D. Hyde \& McKenzie

Distoseptispora K.D. Hyde et al. 2016 (H)*

Jobellisiales M.J. D'souza \& K.D. Hyde

Magnaporthales Thongk. et al.

Ceratosphaeriaceae Z.L. Luo et al.

Ceratosphaeria Niessl $1876(\mathbf{H})^{\# * *}$

Magnaporthaceae P.F. Cannon 
Bifusisporella R.M.F. Silva et al. 2019 (H)

Budhanggurabania P. Wong et al. $2015(\mathbf{H})^{\# *}$

Bussabanomyces Klaubauf et al. 2014 (H)

Ceratosphaerella Huhndorf et al. $2008(\mathbf{H})^{\# * *}$

Clasterosporium Schwein $1832(\mathbf{H})^{\# *}$

Falciphora J. Luo et al. 2015 (H)

Falciphoriella M. Hern.-Restr. \& Crous 2016 (H)

Gaeumannomycella M. Hern.-Restr. \& Crous 2016 (H)

Gaeumannomyces Arx \& D.L. Olivier $1952(\mathbf{H})^{\# *}$

Kohlmeyeriopsis Klaubauf et al. $2014(\mathbf{H})^{\# *}$

Magnaporthiopsis J. Luo et al. $2013(\mathbf{H})^{\# *}$

Mycoleptodiscus Ostaz. 1968 (H)

Omnidemptus P.F. Cannon \& Alcorn 1994 (H) ${ }^{\# *}$

Pseudophialophora J. Luo \& N. Zhang 2014 (H)

Pyriculariopsis M.B. Ellis 1971 (H)

Slopeiomyces Klaubauf et al. 2014 (H)

Pyriculariaceae Klaubauf et al.

Bambusicularia Klaubauf et al. 2014 (H)

Barretomyces Klaubauf et al. 2014 (H)

Deightoniella S. Hughes 1952 (H)

Macgarvieomyces Klaubauf et al. 2014 (H)

Neocordana Hern.-Rest. et al. 2015 (H)

Proxipyricularia Klaubauf et al. 2014 (H)

Pseudopyricularia Klaubauf et al. 2014 (H)

Pyricularia Sacc. 1880 (H)

Pyriculariomyces Y. Marín et al. 2019 (H)

Xenopyricularia Klaubauf et al. 2014 (H)

\section{Myrmecridiales Crous}

Myrmecridiaceae Crous

Myrmecridium Arzanlou et al. $2007(\mathbf{H})^{\# *}$

Neomyrmecridium Crous 2018 (H)

\section{Xenodactylariaceae Crous}

Xenodactylaria Crous 2018 (H)

Ophiostomatales Benny \& Kimbr.

Kathistaceae Malloch \& M. Blackw.

Termitariopsis M. Blackw. et al. 1980 (H)

\section{Ophiostomataceae Nannf.}

Afroraffaelea C.C. Bateman et al. 2016 (H)

Aureovirgo J.A. van der Linde et al. 2016 (H) ${ }^{\# *}$

Ceratocystiopsis H.P. Upadhyay \& W.B. Kendr. 1975 (H) ${ }^{\# *}$

Fragosphaeria Shear 1923 (H) ${ }^{\# *}$

Graphilbum H.P. Upadhyay \& W.B. Kendr. 1975 (H) ${ }^{\# *}$

Hawksworthiomyces Z.W. de Beer et al. 2016 (H)

Leptographium Lagerb. et al. 1927 (H)

Ophiostoma Syd. et al. $1919(\mathbf{H})^{\# *}$

Raffaelea Arx et al. 1965 (H)

Sporothrix Hektoen et al. $1901(\mathbf{H})^{\# *}$ 
Spumatoria Massee et al. 1901 (H) ${ }^{\# *}$

Subbaromyces Hesselt. 1953 (H) ${ }^{\# *}$

Pararamichloridiales Crous

Pararamichloridiaceae Crous

Pararamichloridium Crous 2017 (H)

Phomatosporales Senan. et al.

Phomatosporaceae Senan. \& K.D. Hyde

Phomatospora Sacc. $1875(\mathbf{H})^{\# *}$

Sporidesmiales Crous

Sporidesmiaceae Fr.

Sporidesmium Link $1809(\mathbf{H})^{\# * *}$

Tirisporellales Suetrong et al.

Tirisporellaceae Suetrong et al.

Thailandiomyces Pinruan et al. $2008(\mathbf{H})^{\# *}$

Tirisporella E.B.G. Jones et al. $1996(\mathbf{H})^{\# * *}$

Togniniales Senan. et al.

Togniniaceae Réblová et al.

Phaeoacremonium W. Gams et al. 1996 (H) ${ }^{\# *}$

Xenospadicoidales Hern.-Restr. et al.

Xenospadicoidaceae Hern.-Restr. et al.

Neospadicoides Z.L. Luo 2019 (H)

Spadicoides S. Hughes $1958(\mathbf{H})^{\# *}$

Diaporthomycetidae families incertae sedis

Barbatosphaeriaceae $\mathrm{H}$. Zhang et al.

Barbatosphaeria Réblová 2008 (H) ${ }^{\# *}$

Xylomelasma Réblová 2006 (H) ${ }^{\# *}$

Rhamphoriaceae Réblová

Rhamphoria Niessl 1876 (H) ${ }^{\# *}$

Rhodoveronaea Arzanlou et al. 2018 (H)

Thyridiaceae J.Z. Yue \& O.E. Erikss.

Thyridium Nitschke 1867 (C) $)^{\# *}$

Trichosphaeriaceae G. Winter

Brachysporium Sacc. $1886(\mathbf{H})^{\# *}$

Koorchaloma Subram. $1953(\mathbf{C})^{\# *}$

Woswasiaceae $\mathrm{H}$. Zhang et al.

Xylochrysis Réblová et al. 2014 (C) ${ }^{\# *}$

Diaporthomycetidae families incertae sedis

Sporidesmiella P.M. Kirk 1982 (H)

Subclass Hypocreomycetidae O.E. Erikss. \& Winka 
Coronophorales Nannf.

Ceratostomataceae G. Winter

Dactylidispora Y. Marín et al. 2018 (H) ${ }^{\# *}$

Erythrocarpon Zukal $1886(\mathbf{H})^{\# *}$

Harzia Costantin 1888 (H)

Melanospora Corda $1837(\mathbf{H})^{\# *}$

Microthecium Corda 1842 (H) ${ }^{\# *}$

Chaetosphaerellaceae Huhndorf et al.

Chaetosphaerella E. Müll. et al. 1972 (H) \#*

Crassochaeta Réblová 1999 (H) ${ }^{\# *}$

Falcocladiales R.H. Perera et al.

Falcocladiaceae Somrithipol et al.

Falcocladium S.F. Silveira et al. (1994) (H)

Glomerellales Chadef. ex Réblová et al.

Australiascaceae Réblová \& W. Gams

Monilochaetes Halst. ex Harter $1916(\mathbf{H})^{\# *}$

Glomerellaceae Locq. ex Seifert \& W. Gams

Colletotrichum Corda 1831 (C) (** $^{*}$

Malaysiascaceae Tibpromma \& K.D. Hyde

Malaysiasca Crous \& M.J. Wingf. $2016(\mathbf{H})^{\# *}$

Plectosphaerellaceae W. Gams et al.

Acremoniisimulans Tibpromma et al. 2018 (H)

Acrostalagmus Corda 1838 (H)

Brunneochlamydosporium Giraldo López \& Crous 2018 (H)

Brunneomyces A. Giraldo et al. 2017 (H)

Chlamydosporiella Giraldo López \& Crous2019 (H)

Chordomyces Bilanenko et al. 2015 (H)

Furcasterigmium Giraldo López \& Crous 2019 (H)

Fuscohypha Giraldo López \& Crous 2019 (H)

Gibellulopsis Bat. et al. 1959 (H)

Lectera P.F. Cannon 2012 (C)

Longitudinalis Tibpromma et al. 2017 (H)

Musicillium Zare et al. 2007 (H)

Musidium Giraldo López \& Crous 2019 (H)

Nigrocephalum Giraldo López \& Crous 2019 (H)

Paragibellulopsis Giraldo López \& Crous 2019 (H)

Paramusicillium Giraldo López \& Crous 2019 (H)

Phialoparvum Giraldo López \& Crous 2019 (H)

Plectosphaerella Kleb. 1929 (H)

Sayamraella Giraldo López \& Crous 2019 (H)

Sodiomyces A.A. Grum-Grzhim. et al. 2019 (H) ${ }^{\# *}$

Stachylidium Link 1809 (H)

Summerbellia Giraldo López \& Crous 2019 (H)

Theobromium Giraldo López \& Crous 2019 (H)

Verticillium Nees 1816 (H) 
Reticulascaceae Réblová

Blastophorum Matsush 1971 (H)

Cylindrotrichum Bonord. 1851 (H) ${ }^{\# *}$

Kylindria DiCosmo et al. 1983 (H)

Sporoschismopsis Hol-Jech. et al. $1972(\mathbf{H})^{\# *}$

Glomerellales genus incertae sedis

Ascocodinaea Samuels et al. (H) ${ }^{\# *}$

\section{Hypocreales Lindau}

Bionectriaceae Samuels \& Rossman

Acremonium Link 1809 (H)

Bryocentria Döbbeler 2004 (H)

Bullanockia Crous 2016 (H)

Clonostachys Corda $1839(\mathbf{H})^{\# *}$

Dimerosporiella Speg. 1908 (H)

Fusariella Sacc. 1884 (H)

Geosmithia Pitt 1979 (H)

Gliomastix Guég. 1905 (H)

Heleococcum P.M. Jørg. 1922 (H) ${ }^{\# *}$

Ijuhya Starbäck $1899(\mathbf{H})^{\# *}$

Lasionectria (Sacc.) Cooke 1884 (H)

Lasionectriella Lechat \& J. Fourn. 2016 (H) ${ }^{\# *}$

Mycoarachis Malloch et al. 1970 (H)

Mycocitrus Möller 1901 (H)

Nectriella Nitschke et al. 1870 (H)

Nectriopsis Maire 1911 (H)

Nigrosabulum Malloch et al. 1970 (H)

Paracylindrocarpon Crous et al. 2016 (H)

Periantria Döbbeler \& P.G. Davison 2018 (H) ${ }^{\# *}$

Pronectria Clem. 1931 (H)

Protocreopsis Yoshim Doi $1977(\mathbf{H})^{\# *}$

Rhopalocladium Schroers et al. 1999 (H)

Roumegueriella Speg. 1980 (H) ${ }^{\# *}$

Selinia P. Karst. 1876 (H)

Stilbocrea Pat. 1900 (H)

Stromatonectria Jaklitsch \& Voglmayr 2011 (C)

Synnemellisia N.K. Rao et al. 1989 (H)

Verrucostoma Hirooka et al. 2010 (H)

Xanthonectria J. Fourn. \& P.-A. Moreau 2016 (H) ${ }^{\# *}$

Calcarisporiaceae Jing Z. Sun et al.

Calcarisporium Preuss 1851 (H)

Clavicipitaceae (Lindau) Earle ex Rogerson

Aschersonia Mont. 1848 (C) \#* $^{*}$

Atkinsonella Diehl 1950 (C) $)^{\# *}$

Balansia Speg. $1885(\mathbf{C})^{\# *}$

Claviceps Tul. $1853(\mathbf{C})^{\# *}$

Collarina Giraldo et al. 2014 (H)

Corallocytostroma Y.N. Yu et al. 1980 (C)

Epichloë (Fr.) Tul. et al. (1865) (H) 
Helicocollum Luangsa-ard 2017 (H)

Heteroepichloë E. Tanaka et al. $2002(\mathbf{C})^{\# *}$

Loculistroma F. Patt et al. $1910(\mathbf{C})^{\# *}$

Metapochonia Kepler et al. 2014 (C)

Metarhiziopsis D.W. Li et al. 2008 (C)

Metarhizium Sorokīn $1879(\mathbf{C})^{\# *}$

Moelleriella Bres. 1987 (C) \#** $^{\# *}$

Mycophilomyces Crous et al. 2016 (C)

Myriogenospora G.F. Atk. 1894 (C) (** $^{*}$

Neobarya Lowen 1996 (C) ${ }^{\# *}$

Neocordyceps Kobayasi $1984(\mathbf{C})^{\# *}$

Nigelia Luangsa-ard et al. 2017 (C) \#* $^{*}$

Nigrocornus Ryley et al. 2003 (C) \#* $^{\#}$

Pochonia Bat. et al. 1965 (C) \#* $^{*}$

Pseudomeria G.L. Barron 1980 (H)

Regiocrella Chaverri et al. 2005 (C) (* $^{*}$

Rotiferophthora G.L. Barron 1991 (H)

Samuelsia Chaverri \& K.T. Hodge $2008(\text { C })^{\# *}$

Tyrannicordyceps Kepler et al. $2012(\mathbf{C})^{\# *}$

Ustilaginoidea Bref. 1895 (H)

Cordycipitaceae Kreisel ex G.M. Sung et al.

Akanthomyces Lebert $1858(\mathbf{H})^{\# *}$

Amphichorda Fr. 1825 (H)

Beauveria Vuill. 1912 (H) ${ }^{\# *}$

Beejasamuha Subram. et al. 1977 (H)

Blackwellomyces Spatafora \& Luangsa-ard 2017 (H) ${ }^{\# *}$

Cordyceps (Fr.) Link 1818 (H) ${ }^{\# *}$

Coremiopsis Sizova et al. 1957 (H)

Engyodontium de Hoog 1978 (H)

Gibellula Cavara 1894 (H) ${ }^{\# *}$

Hevansia Luangsa-ard et al. 2017 (H)

Hyperdermium J. White et al. $2000(\mathbf{H})^{\# *}$

Leptobacillium Zare \& W. Gams 2016 (H)

Parengyodontium C.C. Tsang 2016 (H)

Samsoniella Mongkols. et al. $2018(\mathbf{H})^{\# *}$

Simplicillium W. Gams et al. 2001 (H)

Flammocladiellaceae Crous et al.

Flammocladiella Crous et al. 2015 (C) \#* $^{\text {* }}$

\section{Hypocreaceae De Not.}

Arachnocrea Z. Moravec. $1956(\mathbf{H})^{\# * *}$

Escovopsioides H.C. Evans et al. 2012 (H)

Escovopsis J.J. Muchovej et al. 1990 (H)

Hypomyces (Fr.) Tul. et al. $1860(\mathbf{H})^{\# *}$

Kiflimonium Summerb. et al. 2018 (H)

Mycogone Link 1809 (H)

Protocrea Petch $1937(\mathbf{H})^{\# *}$

Sepedonium Link 1809 (H)

Sphaerostilbella (Henn.) Sacc. et al. $1905(\mathbf{H})^{\# *}$

Sporophagomyces K. Põldmaa et al. $1999(\mathbf{H})^{\# *}$ 
Stephanoma Wallr. 1833 (H)

Trichoderma Pers. 1794 (H) ${ }^{\# *}$

Verticimonosporium Matsush. 1971 (H)

Myrotheciomycetaceae Crous

Emericellopsis J.F.H. Beym 1940 (H) ${ }^{\# *}$

Leucosphaerina Arx 1987 (H) ${ }^{\# *}$

Myrotheciomyces Crous 2018 (H)

Trichothecium Link 1809 (H)

Nectriaceae Tul. \& C. Tul.

Aquanectria L. Lombard \& Crous $2015(\mathbf{H})^{\# * *}$

Atractium Link 1809 (H)

Bisifusarium L. Lombard et al. 2015 (H)

Calonectria De Not. $1867(\mathbf{H})^{\# *}$

Calostilbe Sacc. \& Syd. $1902(\mathbf{H})^{\# *}$

Campylocarpon Halleen et al. 2005 (H)

Chaetopsina Rambelli 1956 (H)

Coccinonectria L. Lombard \& Crous $2015(\mathbf{H})^{\# *}$

Corinectria C. González \& P. Chaverri 2017 (H) ${ }^{\# *}$

Corallomycetella Henn. 1904 (H) ${ }^{\# *}$

Corallonectria C. Herrera \& P. Chaverri 2013 (H) \#*

Curvicladiella Decock \& Crous 2006 (H)

Cyanochyta Höhn. 1915 (C)

Cyanophomella Höhn. 1918 (C)

Cylindrocarpostylus R. Kirschner \& Oberw. 1999 (H)

Cylindrocladiella Boesew. $1982(\mathbf{H})^{\# *}$

Cylindrodendrum Bonord. 1851 (H)

Dacryoma Samuels 1988 (H)

Dactylonectria L. Lombard \& Crous $2014(\mathbf{H})^{\# *}$

Dematiocladium Allegr. et al. 2005 (H)

Fusarium Link 1809 (H) ${ }^{\# *}$

Fusicolla Bonord. 1851 (H) ${ }^{\# *}$

Gliocephalotrichum J.J. Ellis \& Hesselt. 1962 (H) ${ }^{\# *}$

Gliocladiopsis S.B. Saksena 1954 (H)

Ilyonectria P. Chaverri \& C. Salgado $2011(\mathbf{H})^{\# *}$

Macroconia (Wollenw.) Gräfenhan et al. 2011 (H)

Mariannaea G. Arnaud ex Samson 1974 (H)

Microcera Desm. 1848 (H)

Murinectria M. Niranjan \& V.V. Sarma $2020(\mathbf{H})^{\# *}$

Nalanthamala Subram. $1956(\mathbf{H})^{\# *}$

Nectria (Fr.) Fr. 1849 (H) ${ }^{\# *}$

Neonectria Wollenw. 1917 (H) ${ }^{\# *}$

Paracremonium L. Lombard \& Crous $2015(\mathbf{H})^{\# *}$

Penicillifer Emden 1968 (H) ${ }^{\#}$

Pleiocarpon L. Lombard \& D. Aiello 2017 (H)

Pseudocosmospora C. Herrera \& P. Chaverri 2013 (H) ${ }^{\# *}$

Pseudoachroiostachys Tibpromma \& K.D. Hyde 2018 (H)

Rectifusarium L. Lombard et al. $2015(\mathbf{H})^{\# *}$

Rugonectria P. Chaverri \& Samuels $2011(\mathbf{H})^{\# *}$

Sarcopodium Ehrenb. 1818 (H)

Thyronectria Sacc. 1875 (C) $)^{\# *}$ 
Varicosporella Lechat \& J. Fourn. $2015(\mathbf{H})^{\# *}$

Volutella Fr. $1832(\mathbf{H})^{\# *}$

Xenoacremonium L. Lombard \& Crous 2015 (H)

Xenocylindrocladium Decock et al. $1997(\mathbf{H})^{\# *}$

Xenoleptographium Marinc. et al. 2015 (H)

Niessliaceae Kirschst.

Eucasphaeria Crous 2007 (C) \#* $^{*}$

Myrtacremonium Crous 2017 (H)

Niesslia Auersw. $1869(\mathbf{H})^{\# *}$

Trichosphaerella E. Bommer et al. 1891 with acremonium-like asexual morph $(\mathbf{H})^{\# *}$

Valetoniellopsis Samuels \& M.E. Barr 1998 with acremonium-like asexual morph $(\mathbf{H})^{\# *}$

Ophiocordycipitaceae G.H. Sung et al.

Drechmeria W. Gams \& H.B. Jansson (H)

Hantamomyces Crous 2020 (H)

Harposporium Lohde $1874(\mathbf{H})^{\# *}$

Ophiocordyceps Petch $1931(\mathbf{H})^{\# *}$

Paraisaria Samson \& B.L. Brady $1983(\mathbf{H})^{\# *}$

Polycephalomyces Kobayasi $1941(\mathbf{H})^{\# *}$

Purpureocillium Luangsa-ard et al. 2011 (H)

Tolypocladium W. Gams $1971(\mathbf{H})^{\# *}$

Sarocladiaceae L. Lombard

Parasarocladium Summerb. et al. Crous 2018 (H)

Stachybotryaceae L. Lombard \& Crous

Achroiostachys L. Lombard \& Crous 2016 (H)

Albifimbria L. Lombard \& Crous 2016 (H)

Albosynnema E.F. Morris 1967 (H)

Alfaria Crous et al. 2014 (C) ${ }^{\# *}$

Alfariacladiella Crous \& R.K. Schumach. 2016 (H)

Brevistachys L. Lombard \& Crous 2016 (H)

Capitofimbria L. Lombard \& Crous 2016 (H)

Cymostachys L. Lombard \& Crous 2016 (H)

Didymostilbe Henn. 1902 (H)

Digitiseta Gordillo \& Decock 2017 (H)

Dimorphiseta L. Lombard \& Crous 2016 (H)

Globobotrys L. Lombard \& Crous 2016 (H)

Grandibotrys L. Lombard \& Crous 2016 (H)

Gregatothecium L. Lombard \& Crous 2016 (H)

Inaequalispora L. Lombard \& Crous 2016 (H)

Hyalinostachys C.G. Lin \& K.D. Hyde 2020 (H)

Kastanostachys L. Lombard \& Crous 2016 (H) ${ }^{\# *}$

Koorchalomella Chona et al. 1958 (H)

Melanopsamma Niessl $1876(\mathbf{H})^{\# *}$

Memnoniella Höhn. 1923 (H)

Myrothecium Tode 1790 (H)

Myxospora L. Lombard \& Crous 2016 (H)

Neomyrothecium L. Lombard \& Crous 2016 (H)

Paramyrothecium L. Lombard \& Crous 2016 (H)

Parasarcopodium Mel'nik et al. 2004 (H) 
Parvothecium L. Lombard \& Crous 2016 (H)

Pseudoornatispora Tibpromma \& K.D. Hyde 2018 (H) (** $^{*}$

Septomyrothecium Matsush. 1971 (H)

Sirastachys L. Lombard \& Crous 2016 (H)

Smaragdiniseta L. Lombard \& Crous 2016 (H)

Stachybotrys Corda $1837(\mathbf{H})^{\# *}$

Striatibotrys L. Lombard \& Crous $2016(\mathbf{H})^{\# *}$

Striaticonidium L. Lombard \& Crous 2016 (H)

Tangerinosporium L. Lombard \& Crous 2016 (H)

Virgatospora Finley 1967 (H)

Xenomyrothecium L. Lombard \& Crous 2016 (H)

Xepicula Nag Raj 1993 (C)

Xepiculopsis Nag Raj 1993 (C)

Tilachlidiaceae Lombard \& Crous

Septofusidium W. Gams 1971 (H)

Tilachlidium Preuss 1851 (H)

Hypocreales genera incertae sedis

Acremoniopsis Giraldo et al. 2014 (H)

Cylindrium Bonord. 1851 (H)

Geosmithia J.Pitt 1979 (H)

Hapsidospora Malloch \& Cain 1970 (C)

Haptospora G.L. Barron 1991 (H)

Harzia Costantin 1888 (H)

Hymenopsis Sacc. 1886 (C)

Illosporiopsis D. Hawksw. 2001 (H)

Illosporium Mart. 1817 (H)

Lichenopenicillus Etayo 2017 (H)

Munkia Speg. 1886 (H)

Neomunkia Petr. 1947 (H)

Neothyronectria Crous \& Thangavel 2016 (C)

Sarocladium W. Gams \& D. Hawksw. 1976 (H)

Saksenamyces A.N. Rai \& P.N. Singh 2018 (H)

Stanjemonium W. Gams et al. 1999 (H)

Stilbella Lindau $1900(\mathbf{H})$

Trichothecium Link 1809 (H)

Microascales Luttr. ex Benny \& Kimbr.

Ceratocystidaceae Locq. ex Réblová et al.

Ambrosiella Brader ex Arx \& Hennebert 1965 (H)

Berkeleyomyces W.J. Nel et al. 2017 (H)

Bretziella Z.W. de Beer et al. $2017(\mathbf{H})^{\# *}$

Ceratocystis Ellis \& Halst. $1890(\mathbf{H})^{\# *}$

Chalaropsis Peyronel 1916 (H)

Davidsoniella Z.W. de Beer et al. $2014(\mathbf{H})^{\# * *}$

Huntiella Z.W. de Beer et al. 2014 (H) ${ }^{\# *}$

Thielaviopsis Went 1893 (H)

Chadefaudiellaceae Faurel \& Schotter ex Benny \& Kimbr.

Faurelina Locq.-Lin. 1975 with arthrographis-like asexual morph $(\mathbf{H})^{\# *}$ 
Gondwanamycetaceae Réblová et al.

Custingophora Stolk et al. 1968 (H)

Graphiaceae De Beer

Graphium Corda 1837 (H)

Halosphaeriaceae E. Müll. \& Arx ex Kohlm.

Cirrenalia Meyers \& R.T. Moore 1960 (H)

Clavatospora Sv. Nilsson ex Marvanová \& Sv. Nilsson 1971 (H)

Corollospora Werderm. $1922(\mathbf{H})^{\# *}$

Sigmoidea J.L. Crane 1968 (H)

Microascaceae Luttr. ex Malloch

Acaulium Sopp 1912 (H)

Brachyconidiellopsis Decock et al. 2004 (H)

Cephalotrichum Link 1809 (H)

Echinobotryum Corda 1831 (H)

Gamsia M. Morelet 1969 (H)

Parascedosporium Gilgado et al. 2007 (H)

Pseudoscopulariopsis M. Sandoval-Denis et al. 2015 (H) ${ }^{\# *}$

Rhinocladium Sacc. \& Marchal 1885 (H)

Scedosporium Sacc. ex Castell. \& Chalm. 1919 (H)

Wardomyces F.T. Brooks \& Hansf. 1923 (H)

Wardomycopsis Udagawa \& Furuya 1978 (H)

Yunnania H.Z. Kong 1998 (H) ${ }^{\# *}$

Triadelphiaceae Y.Z. Lu et al.

Synnematotriadelphia Chuaseehar et al. 2020 (H)

Triadelphia Shearer \& J.L. Crane 1971 (H)

Microascales genera incertae sedis

Cephalotrichiella Crous 2014 (H)

Cornuvesica C.D. Viljoen et al. 2000 with chalara-like asexual morph (H) ${ }^{\# *}$

Sphaeronaemella P. Karst. $1884(\mathbf{H})^{\# *}$

Sporendocladia G. Arnaud ex Nag Raj \& W.B. Kendr. 1975 (H)

Pararamichloridiales Crous

Pararamichloridiaceae Crous

Pararamichloridium Crous 2017 (H)

Torpedosporales E.B.G. Jones et al.

Juncigenaceae E.B.G. Jones et al.

Juncigena Kohlm. et al. 1997 (H)

Torpedosporaceae E.B.G. Jones \& K.L. Pang

Torpedospora Meyers $1957(\mathbf{H})^{\# *}$

Hypocreomycetidae genera incertae sedis

Campylospora Ranzoni 1953 (H)

Dendroclathra Voglmayr \& G. Delgado 2001 (H)

Subclass Lulworthiomycetidae Dayar. et al. 
Lulworthiales Kohlm.

Lulworthiaceae Kohlm.

Cumulospora I. Schmidt 1985 (H)

Halazoon Abdel-Aziz et al. 2010 (C)

Hydea K.L. Pang \& E.B.G. Jones 2010 (H)

Matsusporium E.B.G. Jones \& K.L. Pang 2010 (H)

Moleospora Abdel-Wahab et al. 2010 (H)

Moromyces Abdel-Wahab et al. 2010 (H)

Subclass Savoryellomycetidae Hongsanan et al.

Conioscyphales Réblová \& Seifert

Conioscyphaceae Réblová \& Seifert

Conioscypha Höhn. 1904 (H) ${ }^{\# *}$

Fuscosporellales J. Yang et al.

Fuscosporellaceae J. Yang et al.

Fuscosporella J. Yang et al. 2016 (H)

Mucispora J. Yang et al. 2016 (H)

Parafuscosporella J. Yang \& K.D. Hyde 2016 (H)

Pseudoascotaiwania J. Yang et al. 2016 (H)

Pleurotheciales Réblová \& Seifert

Pleurotheciaceae Réblová \& Seifert

Anapleurothecium Hern.-Restr. et al. 2017 (H)

Melanotrigonum Réblová 2015 (H) ${ }^{\# *}$

Neomonodictys Y.Z. Lu et al. 2020 (H)

Phaeoisaria Höhn. 1909 (H)

Pleurotheciella Réblová et al. 2012 (H) ${ }^{\# *}$

Pleurothecium Höhn. 1919 (H) ${ }^{\# *}$

Sterigmatobotrys Oudem. 1886 (H)

Savoryellales Boonyuen et al.

Savoryellaceae Jaklitsch \& Réblová

Ascotaiwania Sivan. \& H.S. Chang 1992 (H) ${ }^{\# *}$

Canalisporium Nawawi \& Kuthub. 1989 (H) (H* $^{\#}$

Kaseifertia Réblová et al. 2020 (C)

Monotosporella S. Hughes 1958 (H)

Subclass Sordariomycetidae O.E. Erikss. \& Winka

Cephalothecales Maharachch. \& K.D. Hyde

Cephalothecaceae Höhn.

Victoriomyces D. Davolos et al. 2019 (C)

Chaetosphaeriales Huhndorf et al.

Chaetosphaeriaceae Réblová et al.

Adautomilanezia Gusmão et al. 2016 (H)

Anacacumisporium Y.R. Ma \& X.G. Zhang 2016 (H)

Brunneodinemasporium Crous \& R.F. Castañeda 2012 (C)

Catenularia Grove 1886 (H)

Chaetosphaeria Tul. \& C. Tul. 1863 with phialophora-like asexual morph $(\mathbf{H})^{\# *}$

Chaetosphaerides Matsush. 2003 ramichloridium-like asexual morph $(\mathbf{H})^{\# *}$

Chloridium Link 1809 (H) ${ }^{\# *}$ 
Codinaea Maire 1937 (H)

Conicomyces R.C. Sinclair et al. 1983 (H)

Craspedodidymum Hol.-Jech. 1972 (H)

Cryptophiale Piroz. 1968 (H)

Dendrophoma Sacc. 1880 (C)

Dictyochaeta Speg. 1923 (H)

Dinemasporium Lév. 1846 (C)

Eucalyptostroma Crous \& M.J. Wingf. 2016 (C)

Exserticlava S. Hughes 1978 (H)

Gonytrichum Nees \& T. Nees 1818 (H)

Hemicorynespora M.B. Ellis 1972 (H)

Infundibulomyces Plaingam et al. 2003 (C)

Kionochaeta P.M. Kirk \& B. Sutton 1986 (H)

Menispora Pers. $1822(\mathbf{H})^{\# *}$

Menisporopsis S. Hughes $1952(\mathbf{H})^{\# *}$

Neopseudolachnella A. Hashim. \& Kaz. Tanaka 2015 (C)

Paragaeumannomyces Matsush. 2003 (H) ${ }^{\# *}$

Pseudobotrytis Krzemien. \& Badura 1954 (H)

Pseudodinemasporium A. Hashim. \& Kaz. Tanaka 2015 (C)

Pseudolachnea Ranoj. 1910 (C)

Pseudolachnella Teng 1936 (C)

Pyrigemmula D. Magyar \& R. Shoemaker 2011 (H)

Rattania Prabhugaonkar \& Bhat 2009 (H)

Sporoschisma Berk. \& Broome $1847(\mathbf{H})^{\# *}$

Striatosphaeria Samuels \& E. Müll. $1979(\mathbf{H})^{\# *}$

Tainosphaeria F.A. Fernández \& Huhndorf 2005 (H)

Thozetella Kuntze 1891 (H)

Verhulstia Hern.-Rest. 2017 (H)

Zanclospora S. Hughes \& W.B. Kendr. 1965 (H)

Helminthosphaeriaceae Samuels et al.

Diplococcium Grove 1885 (H)

Endophragmiella B. Sutton 1973 (H)

Ruzenia O. Hilber 2002 with selenosporella-like asexual morph $(\mathbf{H})^{\# *}$

Linocarpaceae Konta \& K.D. Hyde

Linocarpon Syd. \& P. Syd. 1917 with phialophora-like asexual morph $(\mathbf{H})^{\# *}$

Chaetosphaeriales genera incertae sedis

Calvolachnella Marinc. et al. 2016 (C)

Nawawia Marvanová 1980 (H)

Neonawawia Jing Yang et al. 2018 (H)

Coniochaetales Huhndorf et al. (= Cordanales M. Hern.-Rest. \& Crous)

Coniochaetaceae Malloch \& Cain

Coniochaeta (Sacc.) Cooke $1887(\mathbf{H})^{\# *}$

Barrina A.W. Ramaley 1997 with phialophora-like asexual morph $(\mathbf{H})^{\# *}$

Cordana Preuss $1851(\mathbf{H})^{\# *}$

Rhexodenticula W.A. Baker \& Morgan-Jones 2001 (H)

Meliolales Gäum. ex D. Hawksw. \& O.E. Erikss.

Meliolaceae G.W. Martin ex Hansf. 
Asteridiella McAlpine $1897(\mathbf{H})^{\# *}$

Cryptomeliola S. Hughes \& Piroz. 1997 (H)

Endomeliola S. Hughes \& Piroz. $1994(\mathbf{H})^{\# *}$

Irenopsis F. Stevens $1927(\mathbf{H})^{\# *}$

Meliola Fr. $1825(\mathbf{H})^{\# *}$

Phyllachorales M.E. Barr

Phyllachoraceae Theiss. \& H. Syd.

Cyclodomus Höhn. 1909 (C)

Diachora Müll. 1893 (C) \#* $^{\#}$

Linochora Höhn. 1910 (C)

Mycohypallage B. Sutton 1963 (C)

Neophyllachora Dayar. \& K.D. Hyde 2017 (C) \#* $^{* *}$

Pseudothiella Petr. 1928 (C) ${ }^{\# * *}$

Rhodosticta Woron. 1911 (C)

Pseudodactylariales Crous

Pseudodactylariaceae Crous

Pseudodactylaria Crous 2017 (H)

Sordariales Chad. ex D. Hawksw. \& O.E. Erikss.

Chaetomiaceae G. Winter

Allobotryotrichum M. Raza \& L. Cai 2019 (H)

Arxotrichum A. Nováková \& M. Kolařik 2018 (H) \#* $^{*}$

Chaetomidium (Zopf) Sacc. 1882 with phialophora-like asexual morph (H) ${ }^{\# *}$

Chaetomium Kunze 1817 with papulaspora-like asexual morph $(\mathbf{H})^{\# * *}$

Crassicarpon Y. Marín et al. $2015(\mathbf{H})^{\# *}$

Madurella Brumpt 1905 (H)

Myceliophthora Costantin 1892 (H)

Staphylotrichum J. Mey. \& Nicot 1957 (H)

Thermothelomyces Y. Marín et al. 2015 (H) ${ }^{\# *}$

Thermothielavioides X. Wei Wang \& Houbraken 2019 (H)

\section{Lasiosphaeriaceae Nannf.}

Cercophora Fuckel $1870(\mathbf{H})^{\# *}$

Eosphaeria Höhn. 1917 with phialophora-like asexual morph $(\mathbf{H})^{\# *}$

Lasiosphaeria Ces. \& De Not. 1863 with phialophora-like asexual morph $(\mathbf{H})^{\# *}$

Mammaria Ces. ex Rabenh. 1854 (H)

Naviculisporaceae Y. Marin \& Stchigel

Areotheca Y. Marin \& Stchigel $2020(\text { C })^{\# *}$

Naviculispora Stchigel et al. 2020 (C)

Podosporaceae X. Wei Wang \& Houbraken

Cladorrhinum Sacc. \& Marchal 1885 (H)

Podospora Ces. 1856 with phialophora-like asexual morph $(\mathbf{H})^{\# *}$

Schizotheciaceae Y. Marin \& Stchigel

Jugulospora N. Lundq. $1972(\mathbf{H})^{\# *}$

Lundqvistomyces Y. Marin \& Stchigel $2020(\mathbf{H})^{\# *}$

Pseudoschizothecium Y. Marín et al. 2020 (H) ${ }^{\# *}$

Schizothecium Corda 1838 (H) ${ }^{\# *}$ 
Sordariaceae G. Winter

Neurospora Shear \& B.O. Dodge 1927 (H)

Sordariales genera incertae sedis

Ascolacicola Ranghoo \& K.D. Hyde 1998 with trichocladium-like asexual morph $(\mathbf{H})^{\# *}$

Cancellidium Tubaki 1975 (H)

Lasiosphaeris Clem. 1909 (H) ${ }^{\# *}$

Lunulospora Ingold 1942 (H)

Ramophialophora M. Calduch et al. 2004 (H)

Remersonia Samson \& Seifert 1997 (H)

Ypsilonia Lév. 1846 (H)

Sordariomycetidae family incertae sedis

Cephalothecaceae Höhn.

Albertiniella Kirschst. 1936 with acremonium-like asexual morph $(\mathbf{H})^{\# *}$

Phialemonium W. Gams \& McGinnis 1983 (H)

Sordariomycetidae genera incertae sedis

Acrodictys M.B. Ellis 1961 (H)

Cryptophyllachora L. Kiss et al. 2018 (H)

Myxocephala G. Weber et al. 1989 (H)

Subclass Xylariomycetidae sensu O.E. Erikss. \& Winka

Amphisphaeriales D. Hawksw. \& O.E. Erikss.*

Amphisphaeriaceae G. Winter

Amphisphaeria Ces. \& De Not. 1863 (C) $)^{\# *}$

Labridella Brenckle 1929 (C) ${ }^{\# *}$

Apiosporaceae K.D. Hyde et al.

Arthrinium Kunze $1817(\mathbf{C})^{\# *}$

Endocalyx Berk. \& Broome 1876 (H)

Clypeophysalosporaceae Giraldo \& Crous

Bagadiella Cheew. \& Crous 2009 (H)

Neophysalospora Crous \& M.J. Wingf. 2014 (C) (** $^{\#}$

Plectosphaera Theiss. 1917 (H)

Hyponectriaceae Petr.

Discosphaerina Höhn 1917 (C or H) ${ }^{\# *}$

Phlogicylindriaceae Senan. \& K.D. Hyde

Phlogicylindrium Crous et al. 2006 (H)

Idriellomyces Crous 2018 (H)

Pseudotruncatellaceae Crous

Pseudotruncatella R.H. Perera et al. 2018 (C)

Sporocadaceae Corda

Annellolacinia B. Sutton 1964 (C)

Allelochaeta Petr. 1955 (C)

Bartalinia Tassi 1900 (C)

Broomella Sacc 1883 (C)

Ciliochorella Syd. 1935 (C) 
Dilophospora Desm. 1840 (C)

Discosia Lib. 1837 (C) \#**

Distononappendiculata F. Liu et al. 2018 (C)

Diversimediispora F. Liu et al. 2018 (C)

Doliomyces Steyaert 1961 (C)

Dyrithiopsis L. Cai et al. 2003 (C)

Heterotruncatella F. Liu et al. 2018 (C)

Hyalotiella Papendorf 1967 (C)

Immersidiscosia Kaz. Tanaka et al. 2010 (C)

Monochaetia (Sacc.) Allesch. 1902 (C)

Morinia Berl. \& Bres. 1889 (C)

Neopestalotiopsis Maharachch. et al. 2014 (C)

Neotruncatella Hyang B. Lee \& T.T.T. Nguyen 2016 (C)

Nonappendiculata F. Liu et al. 2018 (C)

Nothoseiridium Crous 2020 (C)

Parabartalinia F. Liu et al. 2018 (C)

Pestalotiopsis Steyaert 1949 (C)

Pseudopestalotiopsis Maharachch. et al. 2014 (C)

Pseudosarcostroma F. Liu et al. 2018 (C)

Robillarda Sacc. 1880 (C)

Sarcostroma Cooke 1871 (C)

Seimatosporium Corda 1833 C) \#* $^{*}$

Seiridium Nees 1816 (C)

Truncatella Steyaert 1949 (C)

Xenoseimatosporium F. Liu et al. 2018 (C)

Zetiasplozna Nag Raj 1993 (C)

Delonicicolales R.H. Perera et al.

Delonicicolaceae R.H. Perera et al.

Delonicicola R.H. Perera et al. 2017 (C)

Xylariales Nannf.

Anungitiomycetaceae Crous

Anungitiomyces Crous 2019 (H)

Beltraniaceae Nann.

Beltrania Penz. 1882 (H)

Beltraniella Subram. 1952 (H)

Hemibeltrania Piroz. 1963 (H)

Parapleurotheciopsis P.M. Kirk 1982 (H)

Porobeltraniella Gusmão 2004 (H)

Pseudobeltrania Henn. 1902 (H)

Subramaniomyces Varghese \& V.G. Rao 1980 (H)

Subsessila C.G. Lin \& K.D. Hyde 2017 (H)

Castanediellaceae Hern.-Restr. et al.

Castanediella Hern.-Restr. et al. 2015 (H)

Diatrypaceae Nitschke

Cryptovalsa Ces. \& De Not. ex Fuckel $1870(\text { C })^{\# *}$

Diatrype Fr. 1849 (C) \#* $^{\# *}$

Halocryptosphaeria Dayar. et al. 2020 (C) $)^{\# *}$ 
Halodiatrype Dayar. \& K.D. Hyde $2016(\text { C })^{\# *}$

Neoeutypella M. Raza et al. 2019 (H) ${ }^{\# *}$

Graphostromataceae M.E. Barr et al.

Biscogniauxia Kuntze $1891(\mathbf{H})^{\# *}$

Camillea Fr. $1849(\mathbf{H})^{\# *}$

Graphostroma Piroz. 1974 with nodulisporium-like asexual morph $(\mathbf{H})^{\# *}$

Obolarina Pouzar 1986 with rhinocladiella-like asexual morph $(\mathbf{H})^{\# *}$

\section{Hypoxylaceae DC}

Natonodosa Heredia et al. 2020 (H)

Lopadostomataceae Daranag. \& K.D. Hyde

Creosphaeria Theiss. $1910(\mathbf{C} \text { or } \mathbf{H})^{\# *}$

Lopadostoma (Nitschke) Traverso 1906 with libertella-like asexual morph $(\mathbf{C})^{\# *}$

Microdochiaceae Hern.-Restr. et al.

Idriella P.E. Nelson \& S. Wilh. 1956 (H)

Microdochium Syd. 1924 (H) ${ }^{\# * *}$

Selenodriella R.F. Castañeda \& W.B. Kendr. 1990 (H)

Nothodactylariaceae Crous

Nothodactylaria Crous 2019 (H)

Polystigmataceae Höhn. ex Nannf.

Polystigma DC. 1815 (C)

Xyladictyochaetaceae Crous \& Hern.-Restr

Xyladictyochaeta Hern.-Restr. et al. 2017 (H)

Xylariaceae Tul. \& C. Tul.

Abieticola Hyang B. Lee 2017 (H)

Annulohypoxylon Y.M. Ju et al. 2005 (H) ${ }^{\# *}$

Ascotricha Berk. $1838(\mathbf{H})^{\# *}$

Brunneiperidium Daranag. et al. 2015 (H) ${ }^{\# *}$

Catenuliconidia N.G. Liu \& K.D. Hyde 2020 (H)

Collodiscula I. Hino \& Katum. $1955(\mathbf{H})^{\# *}$

Coniolariella D. Garciá et al. with rhinocladiella-like asexual morph $(\mathbf{H})^{\# *}$

Cryptostroma P.H. Greg. \& S. Waller 1952 (H)

Daldinia Ces. \& De Not. $1863(\mathbf{H})^{\# *}$

Diabolocovidia Crous 2020 (H)

Emarcea Duong et al. $2004(\mathbf{H})^{\# *}$

Entalbostroma J.D. Rogers \& P.R. Johnst. 2017 (C) \#* $^{*}$

Halorosellinia Whalley et al. 2000 with geniculosporium-like asexual morph $(\mathbf{H})^{\# *}$

Hypocreodendron Henn. 1897 (H)

Hypoxylon Bull. $1791(\mathbf{H})^{\# *}$

Jumillera J.D. Rogers et al. 1997 with geniculosporium-like and libertella-like asexual morph

$(\mathbf{C})$ or $(\mathbf{H})^{\# *}$

Kretzschmaria Fr. 1849 with geniculosporium-like asexual morph $(\mathbf{H})^{\# *}$

Kretzschmariella Viégas 1944 with mirandina-like asexual morph $(\mathbf{H})^{\# *}$

Libertella Desm. 1830 (C)

Lunatiannulus Daranag. et al. 2015 (C) \#* $^{\text {H }}$ 
Nemania Gray $1821(\mathbf{H})^{\# *}$

Poronia Willd. $1787(\mathbf{H})^{\# *}$

Rhopalostroma D. Hawksw. 1977 with nodulosporium-like asexual morph (H) ${ }^{\# *}$

Rosellinia De Not. 1844 (H) \#* $^{\text {\# }}$

Rostrohypoxylon J. Fourn. \& M. Stadler 2010 with sporothrix-like asexual morph (H) ${ }^{\# *}$

Ruwenzoria J. Fourn. et al. 2010 with nodulosporium-like or sporothrix-like asexual morph

$(\mathbf{H})^{\# *}$

Surculiseries Okane et al. 2001 (H)

Vamsapriya Gawas \& Bhat 2006 (H)

Virgaria Nees $1816(\mathbf{H})^{\# *}$

Xylaria Hill ex Schrank $1789(\mathbf{H})^{\# *}$

Xylariales genera incertae sedis

Anungitea B. Sutton 1973 (H)

Ascotrichella Valldos. \& Guarro 1988 with humicola-like asexual morph $(\mathbf{H})^{\# *}$

Barrmaelia Rappaz 1995 with libertella-like asexual morph $(\mathbf{C})^{\# *}$

Beltraniopsis Bat. \& J.L. Bezerra 1960 (H)

Guayaquilia R.F. Castañeda et al. 2020 (H)

Hadrotrichum Fuckel 1865 (H)

Idriellopsis Hern.-Restr. \& Crous 2015 (C)

Kirstenboschia Quaedvl. et al. 2013 (C)

Linteromyces Crous 2020 (H)

Paraidriella Hern.-Restr. \& Crous 2015 (H)

Paraphysalospora Crous 2017 (H)

Polyancora Voglmayr \& C. Yule 2006 (H)

Polyscytalum Riess 1853 (H)

Pseudophloeospora Crous \& R.G. Shivas 2010 (C)

Pseudosubramaniomyces Crous 2017 (H)

Roselymyces Fiuza et al. 2018 (H)

Sporidesmina Subram. \& Bhat 1989 (H)

Synnemadiella Crous \& M.J. Wingf. 2016 (H)

Synnemapestaloides T. Handa \& Y. Harada 2004 (H)

Tristratiperidium Daranag. et al. $2016(\mathbf{H})^{\# *}$

Xyladictyochaeta Hern.-Restr. et al. 2017 (H)

Xylariomycetidae families incertae sedis

Cainiaceae J.C. Krug

Cainia Arx \& E. Müll. 1955 (C) \#* $^{\text {* }}$

Vesiculozygosporium Crous 2020 (H)

Xylariomycetidae genus incertae sedis

Pseudosporidesmium K.D. Hyde \& McKenzie 2016 (H)

Sordariomycetes orders incertae sedis

Amplistromatales D'souza et al.

Amplistromataceae Huhndorf et al.

Acidothrix Hujslová \& M. Kolařík 2014 (H)

Amplistroma Huhndorf et al. 2009 with acrodontium-like asexual morph $(\mathbf{H})^{\# *}$

Wallrothiella Sacc. $1882(\mathbf{H})^{\# *}$

Parasympodiellales Hern.-Restr. et al.

Parasympodiellaceae Hern.-Restr. et al. 


\section{Tracyllalales Crous}

Tracyllaceae Crous

Tracylla (Sacc.) Tassi. 1904 (C)

Vermiculariopsiellales Hern.-Restr. et al.

Vermiculariopsiellaceae Hern.-Restr. et al.

Vermiculariopsiella Bender 1932 (H)

Xenospadicoidales Hern.-Restr. et al.

Xenospadicoidaceae Hern.-Restr et al.

Pseudodiplococcium Hern.-Restr. et al. 2017 (H)

Xenospadicoides Hern.-Restr. et al. 2017 (H)

Sordariomycetes families incertae sedis

Junewangiaceae J.W. Xia \& X.G. Zhang

Jennwenomyces Goh \& C.H. Kuo 2020 (H)

Sordariomycetes genera incertae sedis

Botryosporium Corda 1831 (H)

Calcarisporium Preuss 1851 (H)

Cryptomycella Höhn. 1925 (C)

Didymobotryum Sacc. 1886 (H)

Ellisembia Subram. 1992 (H)

Fantasmomyces DongHyeon Lee et al. $2016(\mathbf{H})^{\# *}$

Hyalotiopsis Punith. 1970 (C) \#* $^{*}$

Liberomyces Pažoutová et al. 2012 (C)

Melanographium Sacc. 1913 (H)

Teracosphaeria Réblová \& Seifert 2007 with phialophora-like asexual morph $(\mathbf{H})^{\# *}$

Muscodor Worapong et al. 2001 (H)

Mycothermus D.O. Natvig et al. 2018 (H)

Neoeriomycopsis Crous \& M.J. Wingf. 2015 (H)

Neoidriella Hern.-Restr. \& Crous 2015 (H)

Neolamproconium Crous \& Akulov 2020 (C)

Nigrospora Zimm. 1902 (H) ${ }^{\# *}$

Paradiplococcium Hern.-Restr. et al. 2017 (H)

Paramicrodochium Hern.-Restr. \& Crous 2015 (H)

Phialemoniopsis Perdomo et al. 2013 (C)

Phomatospora Sacc. 1875 with sporothrix-like asexual morph $(\mathbf{H})^{\# *}$

Pleurophragmium Costantin 1888 (H)

Selenosporella G. Arnaud ex MacGarvie 1969 (H)

Stanjehughesia Subram. 1992 (H) ${ }^{\# *}$

Xenodium Syd. 1935 (H) \#* $^{*}$

Zalerion R.T. Moore \& Meyers 1962 (H)

Class Xylonomycetes R. Gazis \& P. Chaverri

Xylonomycetales R. Gazis \& P. Chaverri

Xylonomycetaceae R. Gazis \& P. Chaverri

Symbiotaphrina Kühlw. \& Jurzitza ex W. Gams \& Arx 1980 (C)

Trinosporium Crous \& Decock 2012 (C)

Xylona Gazis \& P. Chaverri 2012 (C) 
ASCOMYCOTA families incertae sedis

Batistiaceae Samuels \& K.F. Rodrigues Batistia Cif. 1958 (H) ${ }^{\# *}$

Pseudeurotiaceae Malloch \& Cain

Teberdinia Sogonov et al. 2005 (H)

Seuratiaceae Vuill. ex M.E. Barr

Atichia Flot. 1850 (H)

ASCOMYCOTA genera incertae sedis

Abropelta B. Sutton 1986 (C)

Acarella Syd. 1927 (C)

Acarellina Bat. \& H. Maia 1960 (C)

Acaroconium Kocourk. \& D. Hawksw. 2008 (C)

Acarocybe Syd. 1937 (H)

Acarocybella M.B. Ellis 1960 (H)

Acarocybellina Subram. 1992 (H)

Acarocybiopsis J. Mena et al. 1999 (H)

Acaropeltis Petr. 1937 (C)

Acerviclypeatus Hanlin 1990 (C)

Achoropeltis Syd. 1929 (C)

Acleistia Bayl. Ell. 1917 (C)

Acontium Morgan 1902 (H)

Acrodictyella W.A. Baker \& Partr. 2001 (H)

Acrodictyopsis P.M. Kirk 1983 (H)

Acrodontiella U. Braun \& Scheuer 1995 (H)

Acrophialophora Edward 1961 (H)

Acrophragmis Kiffer \& Reisinger 1970 (H)

Acrospeira Berk. \& Broome 1857 (H)

Acrostaurus Deighton \& Piroz. 1972 (H)

Actinocladium Ehrenb. 1819 (H)

Actinotexis Arx 1960 (C)

Actinothecium Ces. 1854 (C)

Actinothyrium Kunze 1823 (C)

Acumispora Matsush. 1980 (H)

Aenigmatospora R.F. Castañeda et al. 1999 (H)

Agaricodochium X.J. Liu 1981 (H)

Agarwalomyces R.K. Verma \& Kamal 1987 (H)

Agrabeeja Subram. 1995 (H)

Agyriella Sacc. 1884 (H)

Agyriellopsis Höhn. 1903 (C)

Ahmadia Syd. 1939 (C)

Ajrekarella Kamat \& Kalani 1964 (C)

Alatosessilispora K. Ando \& Tubaki 1984 (H)

Alciphila Harmaja 2002 (H)

Algonquinia R.F. Castañeda \& W.B. Kendr. 1991 (H)

Alloneottiosporina Nag Raj 1993 (C)

Allothyriella Bat. et al. 1959 (C)

Allothyrina Bat. \& J.L. Bezerra 1964 (C)

Allothyriopsis Bat. et al. 1959 (C)

Alpakesa Subram. \& K. Ramakr. 1954 (C) 
Alpakesiopsis Abbas et al. 2003 (C)

Alveariospora Meir. Silva et al. 2012 (H)

Alveophoma Alcalde 1952 (C)

Alysidiopsis B. Sutton 1973 (H)

Amallospora Penz. 1897 (H)

Amblyosporium Fresen. 1863 (H)

Ameroconium U. Braun \& Zhurb. 2013 (H)

Amerodiscosiella M.L. Farr 1961 (C)

Amerodiscosiellina Bat. \& Cavalc. 1966 (C)

Amerosporiopsis Petr. 1941 (C)

Amerosporium Speg. 1882 (C)

Amerosympodula Matsush. 1996 (H)

Amoenodochium Peláez \& R.F. Castañeda 1996 (H)

Amoenomyces R.F. Castañeda et al. 1996 (H)

Amphichaetella Höhn. 1916 (H)

Amphichordia Fr. 1825 (H)

Amphophialis R.F. Castañeda et al. 1998 (H)

Amphoropycnium Bat. 1963 (C)

Ampullicephala R.F. Castañeda et al. 2009 (H)

Ampulliferina B. Sutton 1969 (H)

Anabahusakala Carmo et al. 2014 (H)

Anacoronospora J.S. Monteiro et al. 2016 (H)

Anacraspedodidymum C.R. Silva et al. 2014 (H)

Anaexserticlava T.S. Santa Izabel et al. 2015 (H)

Anaphysmene Bubák 1906 (C)

Anarhyma M.H. Pei \& Z.W. Yuan 1986 (C)

Anaselenosporella Heredia et al. 2010 (H)

Anaseptoidium R.F. Castañeda et al. 2012 (H)

Anaverticicladus P.O. Costa et al. 2016 (H)

Ancoraspora Mig. Rodr. 1982 (H)

Ancorasporella J. Mena et al. 2017 (H)

Angiopomopsis Höhn. 1912 (C)

Angulimaya Subram. \& Lodha 1964 (H)

Angulospora Sv. Nilsson 1962 (H)

Annellodentimyces Matsush. 1985 (H)

Annellodochium Deighton 1969 (H)

Annellophora S. Hughes 1952 (H)

Annellophorella Subram. 1962 (H)

Annellophragmia Subram. 1963 (H)

Annellospermosporella P.R. Johnst. 1999 (H)

Annellosympodia McTaggart et al. 2007 (H)

Antennatula Fr. ex F. Strauss 1850 (H)

Antennopsis R. Heim 1952 (H)

Anthopsis Fil. March. et al. 1977 (H)

Anthracoderma Speg. 1888 (C)

Antromyces Fresen. 1850 (H)

Anulohypha Cif. 1962 (H)

Anungitopsis R.F. Castañeda \& W.B. Kendr. 1990 (H)

Aoria Cif. 1962 (C)

Aphanofalx B. Sutton 1986 (C)

Apiocarpella Syd. \& P. Syd. 1919 (C)

Apiosporium Kunze 1817 (C) 
Apogloeum Petr. 1954 (C)

Apomelasmia Grove 1937 (C)

Aporellula B. Sutton 1986 (C)

Aposporella Thaxt. 1920 (H)

Apostrasseria Nag Raj 1983 (C)

Arachnophora Hennebert 1963 (H)

Arachnospora R.F. Castañeda et al. 2003 (H)

Arborillus Munt.-Cvetk. \& Gómez-Bolea 1998 (H)

Arborispora K. Ando 1986 (H)

Arcuadendron Sigler \& J.W. Carmich. 1976 (H)

Ardhachandra Subram. \& Sudha 1978 (H)

Argopericonia B. Sutton \& Pascoe 1987 (H)

Aristastoma Tehon 1933 (C)

Arthrobotryum Ces. 1854 (C)

Arthrocristula Sigler et al. 1982 (H)

Arthromoniliphora S.S. Silva et al. 2017 (H)

Arthropycnis Constant. 1992 (C)

Arthrosporium Sacc. 1880 (H)

Arthrowallemia R.F. Castañeda et al. 1998 (H)

Articulophora C.J.K. Wang \& B. Sutton 1982 (H)

Asbolisiomyces Bat. \& H. Maia 1961 (C)

Ascochytopsis Henn. 1905 (C)

Ascochytulina Petr. 1922 (C)

Ascospora Mont. 1849 (C)

Ashtaangam Subram. 1995 (H)

Aspilaima Bat. \& H. Maia 1961 (C)

Astelechia Cif. 1962 (H)

Asterinothyriella Bat. \& Cif. 1959 (C)

Asterinothyrium Bat. et al. 1959 (C)

Asteroconium Syd. \& P. Syd. 1903 (C)

Asteroglobulus Brackel 2011 (C)

Asteromyces F. Moreau \& V. Moreau 1962 (H)

Asteroscutula Petr. 1948 (C)

Asterostomopora Bat. \& H. Maia 1960 (C)

Asterostomopsis Bat. et al. 1959 (C)

Asterostomula Theiss. 1916 (C)

Asterostomulina Bat. et al. 1964 (C)

Astronatelia Bat. \& H. Maia 1962 (C)

Atractilina Dearn. \& Barthol. 1924 (H)

Atrogeniculata J.S. Monteiro et al. 2014 (H)

Atrosetiphiale Matsush. 1995 (H)

Atrosynnema J.W. Xia et al. 2016 (H)

Aurosphaeria Sun J. Lee et al. 2009 (C)

Avesicladiella W.P. Wu et al. 1997 (H)

Avettaea Petr. \& Syd. 1927 (C)

Bachmanniomyces D. Hawksw. 1981 (C)

Bacillopeltis Bat. 1957 (C)

Bactridium Kunze 1817 (H)

Bactrodesmiella M.B. Ellis 1959 (H)

Badarisama Kunwar et al. 1986 (H)

Bahuchashaka Subram. 1978 (H)

Bahugada K.A. Reddy \& Vasant Rao 1984 (H) 
Bahukalasa Subram. \& Chandrash. 1979 (H)

Balaniopsis P.M. Kirk 1985 (H)

Balanium Wallr. 1833 (H)

Barbarosporina Kirulis 1942 (C)

Barnettella D. Rao \& P. Rag. Rao 1964 (C)

Basauxia Subram. 1995 (H)

Basifimbria Subram. \& Lodha 1968 (H)

Batistina Peres 1961 (C)

Beauveriphora Matsush 1975 (H)

Beccopycnidium F. Stevens 1930 (C)

Beejadwaya Subram. 1978 (H)

Belemnospora P.M. Kirk 1981 (H)

Bellulicauda B. Sutton 1967 (C)

Beltramono Rashmi Dubey et al. 2011 (H)

Beltraniomyces Monohar et al. 2003 (H)

Beniowskia Racib. 1900 (H)

Benjpalia Subram. \& Bhat 1989 (H)

Bhadradriella Nagaraju et al. 2011 (H)

Bhadradriomyces Sureshk. et al. 2009 (H)

Bharatheeya D'Souza \& Bhat 2002 (H)

Bhatia W.A. Baker \& Morgan-Jones 2009 (H)

Bibanasiella R.F. Castañeda \& W.B. Kendr. 1991 (H)

Bicoloromyces Heuchert et al. 2015 (H)

Biflagellospora Matsush. 1975 (H)

Biflagellosporella Matsush. 1993 (H)

Bimeris Petr. 1949 (C)

Bioconiosporium Bat. \& J.L. Bezerra 1964 (H)

Biophomopsis Petr. 1931 (C)

Bisbyopeltis Bat. \& A.F. Vital 1957 (C)

Bispora Corda 1837 (H)

Bisporostilbella Brandsb. \& E.F. Morris 1971 (H)

Bisseomyces R.F. Castañeda 1985 (H)

Blastocatena Subram. \& Bhat 1989 (H)

Blastodictys M.B. Ellis 1976 (H)

Blastofusarioides Matsush. 1996 (H)

Blastophorella Boedijn 1937 (H)

Blastophragma Subram. 1995 (H)

Blennoria Moug. \& Fr. 1825 (C)

Blennoriopsis Petr. 1919 (C)

Bleptosporium Steyaert 1961 (C)

Blodgettia Harv. 1858 (H)

Bomplandiella Speg. 1886 (H)

Bostrichonema Ces. 1867 (H)

Botryoderma Papendorf \& H.P. Upadhyay 1969 (H)

Botryodiplodina Dias \& Sousa da Câmara 1954 (C)

Botryomonilia Goos \& Piroz. 1975 (H)

Botryostroma Höhn. 1911 (C)

Brachycephala J.S. Monteiro et al. 2015 (H)

Brachydesmiella G. Arnaud ex S. Hughes 1961 (H)

Brachysporiellina Subram. \& Bhat 1989 (H)

Brachysporiopsis Yanna et al. 2004 (H)

Braunomyces V.A. Melnik \& Crous 2014 (H) 
Brefeldiopycnis Petr. \& Cif. 1932 (H)

Brencklea Petrak 1923 (C)

Brevicatenospora R.F. Castañeda et al. 2006 (H)

Briania D.R. Reynolds 1989 (H)

Briosia Cavara 1888 (H)

Brycekendrickia Nag Raj 1973 (C)

Bryophytomyces Cif. 1953 (H)

Bulbilopycnis Matsush. 1996 (C)

Bulbocatenospora R.F. Castañeda \& Iturr. 2000 (H)

Bullaserpens Bat. et al. 1965 (C)

Cacumisporium Preuss 1851 (H)

Caeruleoconidia to Zhurb. \& Pino-Bodas 2017 (C)

Calcarispora Marvanová \& Marvan 1963 (H)

Calceispora Matsush. 1975 (H)

Callistospora Petr. 1955 (C)

Calocline Syd. 1939 (C)

Calongeomyces D. Hawksw. \& Etayo 2011 (H)

Camaroglobulus Speer 1986 (C)

Camaropycnis E.K. Cash 1945 (C)

Camarosporellum Tassi 1902 (C)

Camarosporiopsis Abbas et al. 2000 (C)

Camposporidium Nawawi \& Kuthub. 1988 (H)

Camptomeris Syd. 1927 (H)

Candelabrum Beverw. 1951 (H)

Candelosynnema K.D. Hyde \& Seifert 1992 (H)

Capitorostrum Bat. 1957 (C)

Capnocheirides J.L. Crane \& S. Hughes 1982 (H)

Capnodiastrum Speg. 1886 (C)

Capnofrasera S. Hughes 2003 (H)

Capnosporium S. Hughes 1976 (H)

Capsicumyces Gamundí et al. 1979 (H)

Carnegieispora Etayo \& F. Berger 2009 (C)

Carrismyces R.F. Castañeda \& Heredia 2000 (H)

Casaresia Gonz. Frag. 1920 (H)

Castanedaea W.A. Baker \& Partr. 2001 (H)

Catenella Bat. \& Peres 1963 (C)

Catenocuneiphora Matsush. 2003 (H)

Catenophora Luttr. 1940 (C)

Catenophoropsis Nag Raj \& W.B. Kendr. 1988 (C)

Catenospegazzinia Subram. 1991 (H)

Catenosubulispora Matsush. 1971 (H)

Catenosynnema Kodsueb et al. 2007 (H)

Catenulaster Bat. \& C.A.A. Costa 1959 (C)

Catinopeltis Bat. \& C.A.A. Costa 1957 (C)

Catinula Lév. 1848 (C)

Cecidiomyces U. Braun \& Zhurb. 2012 (H)*

Ceeveesubramaniomyces Pratibha et al. 2005 (H)

Ceratocladium Corda 1839 (H)

Ceratophorum Sacc. 1880 (H)

Ceratopycnis Höhn. 1915 (C)

Ceratosporella Höhn. 1923 (H)

Ceratosporium Schwein. 1832 (H) 
Cercosperma G. Arnaud ex B. Sutton \& Hodges 1983 (H)

Ceuthodiplospora Died. 1912 (C)

Ceuthosira Petr. 1924 (C)

Ceuthosporella Petr. \& Syd. 1923 (C)

Chaetendophragmia Matsush. 1971 (H)

Chaetoblastophorum Morgan-Jones 1977 (H)

Chaetochalara B. Sutton \& Piroz. 1965 (H)

Chaetocytostroma Petr. 1920 (C)

Chaetodiplis Clem. 1931 (C)

Chaetodiplodina Speg. 1910 (C)

Chaetopeltaster Katum. 1975 (C)

Chaetophiophoma Speg. 1910 (C)

Chaetopsis Grev. 1825 (H)

Chaetopyrena Pass. 1881 (C)

Chaetoseptoria Tehon 1937 (C)

Chaetosticta Petr. \& Syd. 1925 (C)

Chalarodendron C.J.K. Wang \& B. Sutton 1984 (H)

Chalarodes McKenzie 1991 (H)

Chantransiopsis Thaxt. 1914 (H)

Characonidia Bat. \& Cavalc. 1965 (C)

Charomyces Seifert 1987 (H)

Chasakopama Manohar. et al. 2009 (H)

Cheilaria Lib. 1830 (C)

Cheiroidea W.A. Baker \& Morgan-Jones 2009 (H)

Cheiromycella Höhn. 1910 (H)

Cheiromyceopsis Mercado \& J. Mena 1988 (H)

Cheiromyces Berk. \& M.A. Curtis 1857 (H)

Cheiromycina B. Sutton 1986 (H)

Cheiropolyschema Matsush. 1980 (H)

Chiastospora Riess 1852 (C)

Chionomyces Deighton \& Piroz. 1972 (H)

Chithramia Nag Raj 1988 (C)

Chlamydomyces Bainier 1907 (H)

Chlamydopsis Hol.-Jech. \& R.F. Castañe-da 1986 (H)

Choanatiara DiCosmo 1984 (C)

Choreospora Constant. \& R. Sant. 1987 (H)

Chromosporium Corda 1829 (H)

Chrysachne Cif. 1938 (H)

Chrysalidopsis Steyaert 1961 (C)

Chryseidea Onofri 1981 (H)

Cicinnobella Henn. 1904 (C)

Ciferria Gonz. Frag. 1925 (C)

Ciferriella Petr. 1930 (C)

Ciferrina Petr. 1932 (C)

Ciferriopeltis Bat. \& H. Maia 1965 (C)

Ciferrioxyphium Bat. \& H. Maia 1963 (H)

Ciliochora Höhn. 1919 (C)

Ciliophora Petr. 1929 (C)

Ciliophorella Petr. 1940 (C)

Ciliosporella Petr. 1927 (C)

Circinoconiopsis A. Hern.-Gut. 2013 (H)

Circinoconis Boedijn 1942 (H) 
Cissococcomyces Brain 1923 (H)

Civisubramaniania Vittal \& Dorai 1986 (H)

Cladoconidium Bandoni \& Tubaki 1985 (H)

Cladoniicola Diederich et al. 2001 (C)

Cladosporiella Deighton 1965 (H)

Cladosporiopsis S.C. Ren \& X.G. Zhang 2012 (H)

Clasteropycnis Bat. \& Cavalc. 1963 (C)

Clathroconium Samson \& H.C. Evans 1982 (H)

Clauzadeomyces Diederich 1994 (H)

Clavariana Nawawi 1976 (H)

Cleistocystis Sousa da Câmara 1931 (C)

Cleistonium Speer 1986 (C)

Cleistophoma Petr. \& Syd. 1927 (C)

Clypeispora A.W. Ramaley 1991 (C)

Clypeochorella Petr. 1923 (C)

Clypeopatella Petr. 1942 (C)

Clypeophialophora Bat. \& Peres 1962 (C)

Clypeopycnis Petr. 1925 (C)

Clypeoseptoria F. Stevens \& P.A. Young 1925 (C)

Clypeostagonospora Punith. 1981 (C)

Coccogloeum Petr. 1955 (C)

Codonmyces Calat. \& Etayo 1999 (H)

Colemaniella Agnihothr. 1974 (H)

Coleodictyospora Charles 1929 (H)

Coleoseptoria Petr. 1940 (C)

Colispora Marvanová 1988 (H)

Colletoconis de Hoog \& Aa 1978 (C)

Colletosporium Link 1824 (H)

Collostroma Petr. 1947 (C)

Columnodomus Petr. 1941 (C)

Columnothyrium Bubák 1916 (C)

Comatospora Piroz. \& Shoemaker 1971 (C)

Comocephalum Syd. 1939 (C)

Complexipes C. Walker 1979 (H)

Condylospora Nawawi 1976 (H)

Coniambigua Etayo \& Diederich 1995 (C)

Conioscyphopsis Goh \& K.D. Hyde 1998 (H)

Coniothyrina Syd. 1912 (C)

Conjunctospora Udagawa \& Uchiy. 1999 (H)

Conostoma Bat. \& J.L. Bezerra 1965 (C)

Conostroma Moesz 1921 (C)

Consetiella Hol.-Jech. \& Mercado 1982 (H)

Coremiella Bubák \& K. Krieg. 1912 (H)

Cornucopiella Höhn. 1915 (C)

Cornutispora Piroz. 1973 (C)

Cornutostilbe Seifert 1990 (H)

Corynecercospora V.K. Pal et al. 2006 (H)

Corynesporella Munjal \& H.S. Gill 1961 (H)

Corynesporina Subram. 2009 (H)

Corynesporopsis P.M. Kirk 1981 (H)

Costanetoa Bat. \& J.L. Bezerra 1963 (C)

Crandallia Ellis \& Sacc. 1897 (C) 
Craneomyces Morgan-Jones et al. 1987 (H)

Craspedodidimella F.R. Barbosa et al. 2020 (H)

Creodiplodina Petr. 1957 (C)

Creonecte Petr. 1949 (C)

Creoseptoria Petr. 1937 (C)

Creothyriella Bat. \& C.A.A. Costa 1957 (C)

Cribropeltis Tehon 1933 (C)

Crousobrauniella Sh. Kumar et al. 2018 (H)

Crustodiplodina Punith. 1988 (C)

Cryptoceuthospora Petr. 1921 (C)

Cryptocline Petr. 1924 (C)

Cryptocoryneopsis B. Sutton 1980 (H)

Cryptophialoidea Kuthub. \& Nawawi 1987 (H)

Cryptosporium Kunze 1817 (C)

Cryptumbellata Udagawa \& Uchiy. 1999 (H)

Ctenosporium R. Kirschner 2006 (H)

Cubasina R.F. Castañeda 1986 (H)

Culicidospora R.H. Petersen 1960 (H)

Culicinomyces Couch et al. 1974 (H)

Curucispora Matsush. 1981 (H)

Curvulariopsis M.B. Ellis 1961 (H)

Cyanopatella Petr. 1949 (C)

Cyclomarsonina Petr. 1965 (C)

Cylindrogloeum Petr. 1941 (C)

Cylindromyces Manohar. et al. 2004 (H)

Cylindrothyrium Maire 1907 (C)

Cylindroxyphium Bat. \& Cif. 1963 (C)

Cystidiella Malan 1943 (Y)

Cystotricha Berk. \& Broome 1850 (C)

Cytodiscula Petr. 1931 (C)

Cytogloeum Petr. 1925 (C)

Cytonaema Höhn. 1914 (C)

Cytoplacosphaeria Petr. 1920 (C)

Cytosphaera Died. 1916 (C)

Cytosporella Sacc. 1884 (C)

Cyttariella Palm 1932 (C)

Dactylifera Alcorn 1987 (H)

Dactylosporium Harz 1871 (H)

Dasysticta Speg. 1912 (C)

Davisiella Petr. 1924 (C)

Dearnessia Bubák 1916 (C)

Deccanodia Singhai 1974 (C)

Deichmannia Alstrup \& D. Hawksw. 1990 (H)

Delortia Pat. \& Gaillard 1888 (H)

Dendrodomus Bubák 1915 (C)

Dendrographiella Agnihothr. 1972 (H)

Dendrographium Massee 1892 (H)

Dendrospora Ingold 1943 (H)

Dendrosporium Plakidas \& Edgerton ex J.L. Crane 1972 (H)

Dendryphiosphaera Lunghini \& Rambelli 1978 (H)

Dennisographium Rifai 1977 (H)

Denticularia Deighton 1972 (H) 
Dentocircinomyces R.F. Castañeda \& W.B. Kendr. 1990 (H)

Descalsia A. Roldán \& Honrubia 1989 (H)

Desertella Mouch. 1979 (H)

Desmidiospora Thaxt. 1891 (H)

Dexhowardia J.J. Taylor 1970 (H)

Diademospora B.E. Söderstr. \& Bååth 1979 (H)

Diarimella B. Sutton 1980 (C)

Dichelostroma Bat. \& Peres 1963 (C)

Dicholobodigitus G.P. White \& Illman 1988 (H)

Dichotomophthoropsis M.B. Ellis 1971 (H)

Dichotophora Whitton et al. 2012 (H)

Dictyoaquaphila J.S. Monteiro et al. 2016 (H)*

Dictyoarthrinium S. Hughes 1952 (H)

Dictyocatenulata Finley \& E.F. Morris 1967 (H)

Dictyoceratosporella Y.R. Ma \& X.G. Zhang 2016 (H)

Dictyodesmium S. Hughes 1951 (H)

Dictyodochium Sivan. 1984 (H)

Dictyophrynella Bat. \& Cavalc. 1964 (H)

Dictyopolyschema M.B. Ellis 1976 (H)

Dictyorostrella U. Braun 1999 (H)

Dictyospiropes M.B. Ellis 1976 (H)

Dictyotrichocladium Fiuza et al. 2017 (H)

Didymochaetina Bat. \& J.L. Bezerra 1965 (C)

Didymochora Höhn. 1918 (C)

Didymopsis Sacc. \& Marchal 1885 (H)

Didymosporina Höhn. 1916 (C)

Diedickea Syd. \& P. Syd. 1913 (C)

Digicatenosporium S.M. Leão et al. 2015 (H)

Digitodochium Tubaki \& Kubono 1989 (H)

Digitomyces Mercado et al. 2003 (H)

Digitopodium U. Braun et al. 2005 (H)

Digitoramispora R.F. Castañeda \& W.B. Kendr. 1990 (H)

Digitosarcinella S. Hughes 1984 (H)

Dimastigosporium Faurel \& Schotter 1965 (C)

Diplocladiella G. Arnaud ex M.B. Ellis 1976 (H)

Diplodinis Clem. 1931 (C)

Diplodinula Tassi 1902 (C)

Diploplenodomus Died. 1912 (C)

Diplosporonema Höhn. 1917 (C)

Diplozythiella Died. 1916 (C)

Discogloeum Petr. 1923 (C)

Discomycetoidea Matsush. 1993 (H)

Discosiella Syd. \& P. Syd. 1912 (C)

Discosiellina Subram. \& K.R.C. Reddy 1972 (C)

Discosporina Höhn. 1927 (C)

Discotheciella Syd. \& P. Syd. 1917 (C)

Discozythia Petr. 1922 (C)

Dissitimurus E.G. Simmons et al. 1987 (H)

Distophragmia R.F. Castañeda et al. 2015 (H)

Ditangifibula G.C. Adams 1996 (H)

Domingoella Petr. \& Cif. 1932 (H)

Dothideodiplodia Murashk. 1927 (C) 
Dothioropsis Riedl 1974 (C)

Drepanospora Berk. \& M.A. Curtis 1875 (H)

Drudeola Kuntze 1891 (C)

Drumopama Subram. 1957 (H)

Dryosphaera Jørg. Koch \& E.B.G. Jones 1989 (C)

Dualomyces Matsush. 1987 (H)

Dwayabeeja Subram. 1958 (H)

Dwayaloma Subram. 1957 (H)

Dwayalomella Brisson et al. 1975 (C)

Dwibahubeeja N. Srivast. et al. 1995 (H)

Dwibeeja Subram. 1995 (H)

Dwiroopella Subram. \& Muthumary 1986 (C)

Ebollia Minter \& Caine 1980 (C)

Echinocatena R. Campb. \& B. Sutton 1977 (H)

Echinochondrium Samson \& Aa 1975 (H)

Echinoconidiophorum Pereira-Carv. \& Dianese 2009 (H)

Elachopeltella Bat. \& Cavalc. 1964 (C)

Elattopycnis Bat. \& Cavalc. 1964 (C)

Elegantimyces Goh et al. 1998 (H)

Elletevera Deighton 1969 (H)

Ellisembiopsis T.S. Santa Izabel \& Gusmão 2013 (H)

Ellismarsporium R.F. Castañeda \& X.G. Zhang 2017 (H)

Elotespora R.F. Castañeda \& Heredia 2010 (H)

Embryonispora G.Z. Zhao 2013 (H)

Enantioptera Descals 1983 (H)

Endobotrya Berk. \& M.A. Curtis 1874 (C)

Endobotryella Höhn. 1909 (C)

Endoconospora Gjaerum 1971 (H)

Endocoryneum Petr. 1922 (C)

Endocoryneum Petr. 1922 (C)

Endogenospora R.F. Castañeda et al. 2010 (H)

Endomelanconium Petr. 1940 (C)

Endophragmiopsis M.B. Ellis 1966 (H)

Endoplacodium Petr. 1949 (C)

Endoramularia Petr. 1923 (C)

Endosporoideus W.H. Ho et al. 2005 (H)

Endozythia Petr. 1959 (C)

Enerthidium Syd. 1939 (C)

Engelhardtiella A. Funk 1973 (H)

Enridescalsia R.F. Castañeda \& Guarro 1998 (H)

Enthallopycnidium F. Stevens 1925 (C)

Entoderma Hanula et al. 1991 (C)

Epaphroconidia Calat. \& V. Atienza 1995 (C)

Ephelidium C.W. Dodge \& E.D. Rudolph 1955 (C)

Epiclinium Fr. 1849 (C)

Epicoccospora Budathoki \& S.K. Singh 1995 (H)

Epidermidophyton E. Lang 1879 (H)

Epinephroma Zhurb. 2012 (C)

Episporogoniella U. Braun 1994 (H)

Epistigme Syd. 1924 (C)

Epithyrium (Sacc.) Trotter 1931(C)

Eriocercospora Deighton 1969 (H) 
Eriocercosporella Rak. Kumar et al. 1998 (H)

Eriomycopsis Speg. 1910 (H)

Eriospora Berk. \& Broome 1850 (C)

Ernakulamia Subram. 1996 (H)

Erysiphopsis Speg. 1910 (C)

Esteya J.Y. Liou et al. 1999 (H)

Eustilbum Rabenh. 1864 (H)

Evanidomus Caball. 1941 (C)

Everhartia Sacc. \& Ellis 1882 (H)

Everniicola D. Hawksw. 1982 (C)

Eversia J.L. Crane \& Schokn. 1977 (H)

Excipularia Sacc. 1884 (H)

Exophoma Weedon 1926 (C)

Exosporella Höhn. 1912 (H)

Exosporodiella Ganie et al. 2012 (C)

Fairmaniella Petr. \& Syd. 1926 (C)

Favostroma B. Sutton \& E.M. Davison 1983 (C)

Feltgeniomyces Dieder. 1990 (H)

Fenestroconidia Calat. \& Etayo 1999 (H)

Fissuricella Pore et al. 1977 (H)

Flabellocladia Nawawi 1985 (H)

Flabellospora Alas. 1968 (H)

Flosculomyces B. Sutton 1978 (H)

Frigidispora K.D. Hyde \& Goh 1999 (C)

Fujimyces Minter \& Caine 1980 (C)

Fuligomyces Morgan-Jones \& Kamal 1984 (H)

Fumagopsis Speg. 1910 (H)

Funicularius K.K. Baker \& Zaim 1979 (H)

Furcaspora Bonar 1965 (C)

Fusamen (Sacc.) P. Karst. 1890 (C)

Fuscophialis B. Sutton 1977 (H)

Fusichalara S. Hughes \& Nag Raj 1973 (H)

Fusisporella Speg. 1911 (H)

Fusticeps J. Webster \& R.A. Davey 1980 (H)

Gampsonema Nag Raj 1975 (C)

Gangliophora Subram. 1992 (H)

Gangliostilbe Subram. \& Vittal 1976 (H)

Garnaudia Borowska 1977 (H)

Gaubaea Petr. 1942 (C)

Geastrumia Bat. 1960 (C)

Gelatinocrinis Matsush. 1995 (H)

Gelatinopycnis Dyko \& B. Sutton 1979 (C)

Geminoarcus K. Ando 1993 (H)

Gemmulina Descals \& Marvanová 1999 (H)

Geohypha (Fr.) Hennebert 2020 (H)

Gilmaniella G.L. Barron 1964 (H)

Glaphyriopsis B. Sutton \& Pascoe 1987 (C)

Glioannellodochium Matsush. 1989 (H)

Glioblastocladium Matsush. 1989 (H)

Globoconidiopsis G.F. Sepúlveda et al. 2009 (H)

Globoconidium G.F. Sepúlveda et al. 2009 (H)

Gloeocoryneum Weindlm. 1964 (C) 
Gloeodes Colby 1920 (C)

Gloeosporiella Cavara 1892 (H)

Gloiosphaera Höhn. 1902 (H)

Glutinium Fr. 1849 (C)

Goidanichiella G.L. Barron ex W. Gams 2009 (H)

Gonatobotryum Sacc. 1880 (H)

Goniopila Marvanová \& Descals 1985 (H)

Goosiella Morgan-Jones et al. 1986 (H)

Goosiomyces N.K. Rao \& Manohar. 1989 (H)

Grallomyces F. Stevens 1918 (H)

Graphiothecium Fuckel 1870 (H)

Groveolopsis Boedijn 1951 (C)

Guarroa M. Calduch et al. 2020 (H)

Guedea Rambelli \& Bartoli 1978 (H)

Guelichia Speg. 1886 (H)

Gymnoxyphium Cif. et al. 1963 (C)

Gyrothrix (Corda) Corda 1842 (H)

Hadronema Syd. \& P. Syd. 1909 (H)

Hadrosporium Syd. 1938 (H)

Halysiomyces E.G. Simmons 1981 (H)

Hansfordia S. Hughes 1951 (H)

Hansfordiopeltis Bat. \& C.A.A. Costa 1956 (C)

Hansfordiopeltopsis M.L. Farr 1986 (C)

Hapalosphaeria Syd. 1908 (C)

Haplariopsis Oudem. 1903 (H)

Haplobasidion Erikss. 1889 (H)

Haplolepis Syd. 1925 (C)

Haptocara Drechsler 1975 (H)

Harmoniella V.N. Boriss. 1981 (H)

Harpographium Sacc. 1880 (H)

Harpostroma Höhn. 1928 (C)

Hawksworthiana U. Braun 1988 (H)

Heimiodora Nicot 1960 (H)

Helensiella Minter et al. 2015 (H)

Helhonia B. Sutton 1980 (C)

Helicofilia Matsush. 1983 (H)

Helicogoosia Hol.-Jech. 1991 (H)

Helicominopsis Deighton 1960 (H)

Helicorhoidion S. Hughes 1958 (H)

Helicosingula P.S. van Wyk et al. 1985 (H)

Helicothyrium I. Hino \& Katum. 1961 (C)

Helicoubisia Lunghini \& Rambelli 1979 (H)

Heliscella Marvanová 1980 (H)

Heliscina Marvanová 1980 (H)

Helminthosporiomyces G.F. Sepúlveda et al. 2009 (H)

Hemicorynesporella Subram. 1992 (H)

Hemidothis Syd. \& P. Syd. 1916 (C)

Hemisphaeropsis Petr. 1947 (C)

Hendersoniella Tassi 1900 (C)

Hendersonina E.J. Butler 1913 (C)

Hendersoniopsis Höhn. 1918 (C)

Hendersonula Speg. 1880 (C) 
Hendersonulina Petr. 1951 (C)

Henicospora P.M. Kirk \& B. Sutton 1980 (H)

Herposira Syd. 1938 (H)

Herreromyces R.F. Castañeda \& W.B. Kendr. 1991 (H)

Heterocephalum Thaxt. 1903 (H)

Heteroconidium Sawada 1944 (H)

Heterosporiopsis Petr. 1950 (H)

Hexacladium D.L. Olivier 1983 (H)

Himantia Pers. 1801 (?sterile)

Hinoa Hara \& I. Hino 1961 (H)

Hirudinaria Ces. 1856 (H)

Hobsoniopsis D. Hawksw. 2001 (H)

Hoehneliella Bres. \& Sacc. 1902 (C)

Holubovaea Mercado 1983 (H)

Homalopeltis Bat. \& Valle 1961 (C)

Hoornsmania Crous 2007 (H)

Hormiactis Preuss 1851 (H)

Hormiokrypsis Bat. \& Nascim. 1957 (H)

Hormiscioideus M. Blackw. \& Kimbr. 1979 (H)

Hormocephalum Syd. 1939 (H)

Hormographis Guarro et al. 1986 (H)

Hughesinia J.C. Lindq. \& Gamundí 1970 (H)

Humicolopsis Verona 1977 (H)

Hyalobelemnospora Matsush. 1993 (H)

Hyalocamposporium Révay \& J. Gönczöl 2007 (H)

Hyalocephalotrichum Nagaraju et al. 2011 (H)

Hyalocladium Mustafa 1977 (H)

Hyalocylindrophora J.L. Crane \& Dumont 1978 (H)

Hyalodictyum Woron. 1916 (C)

Hyalohelicomina T. Yokoy. 1974 (H)

Hyalopleiochaeta R.F. Castañeda et al. 1996 (H)

Hyalosynnema Matsush. 1975 (H)

Hyalothyridium Tassi 1900 (C)

Hyalotiella Papendorf 1967 (C)

Hydrometrospora J. Gönczöl \& Révay 1985 (H)

Hymenella Fr. 1822 (H)

Hymeniopeltis Bat. 1959 (C)

Hymenobactron (Sacc.) Höhn. 1916 (H)

Hymenopsis Sacc. 1886 (C)

Hyphodiscosia Lodha \& K.R.C. Reddy 1974 (H)

Hyphodiscosioides Matsush. 1993 (H)

Hyphopolynema Nag Raj 1977 (H)

Hyphostereum Pat. 1892 (C)

Hyphothyrium B. Sutton \& Pascoe 1989 (H)

Hyphozyma de Hoog \& M.T. Sm. 1981 (H)

Hypocline Syd. 1939 (C)

Hypodermina Höhn. 1916 (C)

Hypogloeum Petr. 1923 (C)

Hysteridium P. Karst. 1905 (C)

Hysterodiscula Petr. 1942 (C)

Hysteropycnis Hilitzer 1929 (C)

Ialomitzia Gruia 1964 (H) 
Idiocercus B. Sutton 1967 (C)

Imicles Shoemaker \& Hambl. 2001 (H)

Impudentia Vujanović 2003 (H)

Inesiosporium R.F. Castañeda \& W. Gams 1997 (H)

Inifatiella R.F. Castañeda 1985 (H)

Intercalarispora J.L. Crane \& Schokn. 1983 (H)

Ionophragmium Peres 1961 (C)

Irpicomyces Deighton 1969 (H)

Isaria Pers. 1794 (H)

Isariella Henn. 1908 (H)

Ischnostroma Syd. \& P. Syd. 1914 (H)

Isthmoconidium Etayo \& Fr. Berger 2013 (H)*

Isthmolongispora Matsush. 1971 (H)

Isthmophragmospora Kuthub. \& Nawawi 1992 (H)

Isthmospora $\mathrm{F}$. Stevens 1918 (H)

Isthmotricladia Matsush. 1971(H)

Ityorhoptrum P.M. Kirk 1986 (H)

Iyengarina Subram. 1958 (H)

Jahniella Petr. 1921 (C)

Javonarxia Subram. 1995 (H)

Jayarambhatia J. Pratibha 2013 (H)

Jerainum Nawawi \& Kuthub. 1992 (H)

Jubispora B. Sutton \& H.J. Swart 1986 (C)

Junctospora Minter \& Hol.-Jech. 1981 (H)

Junewangia W.A. Baker \& Morgan-Jones 2002 (H)

Kalamarospora G. Delgado 2011 (H)

Kalchbrenneriella Diederich \& M.S. Christ. 2002 (H)

Kaleidosporium Van Warmelo \& B. Sutton 1981 (C)

Kamatella Anahosur 1969 (C)

Kamatia V.G. Rao \& Subhedar 1976 (H)

Kameshwaromyces Kamal et al. 1986 (H)

Karsteniomyces D. Hawksw. 1980 (C)

Kendrickiella K. Jacobs \& M.J. Wingf. 2001 (H)

Ketubakia Kamat et al. 1987 (H)

Kiliophora Kuthub. \& Nawawi 1993 (H)

Kionocephala P.M. Kirk 1986 (H)

Kmetia Bres. \& Sacc. 1902 (H)

Kmetiopsis Bat. \& Peres 1960 (H)

Knemiothyrium Bat. \& J.L. Bezerra 1960 (C)

Kodonospora K. Ando 1993 (H)

Kolletes Kohlm. \& Volkm.-Kohlm. 2005 (H)

Kontospora A. Roldán et al. 1990 (H)

Korunomyces Hodges \& F.A. Ferreira 1981 (H)

Kostermansinda Rifai 1968 (H)

Kostermansindiopsis R.F. Castañeda 1986 (H)

Kramabeeja G.V. Rao \& K.A. Reddy 1981 (H)

Kramasamuha Subram. \& Vittal 1973 (H)

Kreiseliella Braun 1991 (C)

Kumanasamuha P.Rag. Rao \& D. Rao 1964 (H)

Kutilakesa Subram. 1956 (H)

Kyphophora B. Sutton 1991 (C)

Lacellina Sacc. 1913 (H) 
Lacellinopsis Subram. 1953 (H)

Laciniocladium Petri 1917 (H)

Laeviomyces D. Hawksw. 1981 (C)

Lagenomyces Cavalc. \& A.A. Silva 1972 (C)

Lambdasporium Matsush. 1971 (H)

Lambinonia Sérus. \& Diederich 2005 (H)

Laocoön J.C. David 1997 (H)

Lappodochium Matsush. 1975 (H)

Lasiodiplodiella Zambett. 1955 (C)

Lasiophoma Speg. 1918 (C)

Lasiothyrium Syd. \& P. Syd. 1913 (C)

Lasmeniella Petr. \& Syd. 1927 (C)

Latericonis G.V. Rao et al. 1984 (H)

Lateriramulosa Matsush. 1971 (H)

Laterispora Uecker et al. 1982 (H)

Lawalreea Dieder. 1990 (C)

Lecaniocola Brain 1923 (C)

Lecophagus M.W. Dick 1990 (H)

Leeina Petr. 1923 (C)

Leightoniomyces D. Hawksw. \& B. Sutton 1977 (H)

Lembuncula Cif. 1954 (C)

Lemkea Morgan-Jones \& R.C. Sinclair 1983 (H)

Lepisticola W. Gams 2009 (H)

Leprieurinella Bat. \& H. Maia 1961 (C)

Leptochlamys Died. 1921(C)

Leptodermella Höhn. 1915 (C)

Leptomelanconium Petr. 1923 (C)

Leptophyllosticta I.E. Brezhnev 1939 (C)

Leptostromella (Sacc.) Sacc. 1884 (C)

Leptothyrella Sacc. 1885 (C)

Leptothyrina Höhn. 1915 (C)

Leptothyrium Kunze 1823 (C)

Leucodochium Syd. \& P. Syd. 1917 (H)

Leuliisinea Matsush. 1985 (H)

Libertiella Speg. \& Roum. 1880 (H)

Lichenobactridium Diederich \& Etayo 1995 (H)

Lichenodiplisiella S.Y. Kondr. \& Kudra-tov 2002 (C)

Lichenohendersonia Calat. \& Etayo 2001 (C)

Lichenopuccinia D. Hawksw. \& Hafellner 1984 (H)

Lichenostella Calat. \& Etayo 1999 (H)

Linkosia A. Hern. Gut. \& B. Sutton 1997 (H)

Linochorella Syd. \& P. Syd. 1912 (C)

Linodochium Höhn. 1909 (H)

Listeromyces Penz. \& Sacc. 1901 (H)

Lobatopedis P.M. Kirk 1979 (H)

Loliomyces Maire 1937 (H)

Lomaantha Subram. 1954 (H)

Lomachashaka Subram. 1956 (H)

Luxuriomyces R.F. Castañeda 1988 (H)

Luzfridiella R.F. Castañeda \& W.B. Kendr. 1991 (H)

Lylea Morgan-Jones 1975 (H)

Lysotheca Cif. 1962 (C) 
Mackenziella Yanna \& K.D. Hyde 2009 (H)

Macrodiplodia Sacc. 1884 (C)

Macrotrichum Grev. 1825 (H)

Mahabalella B. Sutton \& S.D. Patil 1966 (H)

Manginella Bat. \& H. Maia 1961 (C)

Mapletonia B. Sutton 1991 (C)

Margarinomyces Laxa 1930 (H)

Martinellisia V.G. Rao \& Varghese 1977 (H)

Massalongina Bubák 1916 (C)

Massariothea Syd. 1939 (C)

Matsushimaea Subram. 1978 (H)

Matsushimiella R.F. Castañeda \& Heredia 2001 (H)

Matsushimomyces V.G. Rao \& Varghese 1979 (H)

Medusamyces G.L. Barron \& Szijarto 1990 (H)

Megalodochium Deighton 1960 (H)

Megaloseptoria Naumov 1925 (C)

Melanocephala S. Hughes 1979 (H)

Melanophoma Papendorf \& J.W. du Toit 1967 (C)

Melophia Sacc. 1884 (C)

Menidochium R.F. Castañeda \& W.B. Kendr. 1990 (H)

Mercadinula M. Hernández-Restrepo et al. 2011 (H)

Mercadomyces J. Mena 1988 (H)

Merismella Syd. 1927 (C)

Metadiplodia Syd. 1937 (C)

Metazythia Petr. 1950 (C)

Metazythiopsis M. Morelet 1988 (C)

Microblastosporon Cif. 1930 (H)

Microclava F. Stevens 1917 (H)

Microdiscula Höhn. 1915 (C)

Microdothiorella C.A.A. Costa \& Sousa da Câmara 1955 (C)

Microhendersonula Dias \& Sousa da Câmara 1952 (C)

Microperella Höhn. 1909 (C)

Micropustulomyces R.W. Barreto 1995 (C)

Microtyle Speg. 1919 (C)

Microxyphiella Speg. 1918 (C)

Microxyphiopsis Bat. 1963 (C)

Milospium D. Hawksw. 1975 (H)

Mindoa Petr. 1949 (C)

Minimidochium B. Sutton 1969 (H)

Minteriella Heredia et al. 2013 (H)

Minutophoma D. Hawksw. 1981 (C)

Mirandina G. Arnaud ex Matsush. 1975 (H)

Miricatena Punith. \& Spooner 2011 (H)

Mirimyces Nag Raj 1993 (C)

Mixtoconidium Etayo 1995 (C)

Mohgaonidium Singhai 1974 (C)

Monochaetiella E. Castell. 1943 (C)

Monochaetinula Muthumary et al. 1986 (C)

Monochaetopsis Pat. 1931(H)

Monodia Breton \& Faurel 1970 (C)

Monodidymaria U. Braun 1994 (H)

Monodisma Alcorn 1975 (H) 
Monostichella Höhn. 1916 (C)

Moorella P. Rag. Rao \& D. Rao 1964 (H)

Moralesia Urries 1956 (C)

Morosporium Renault \& Roche 1898 (C)

Morrisographium M. Morelet 1968 (H)

Mucosetospora M. Morelet 1972 (C)

Muiaria Thaxt. 1914 (H)

Muiogone Thaxt. 1914 (H)

Muirella R. Sprague 1959 (H)

Murogenella Goos \& E.F. Morris 1965 (H)

Mycelephas R.F. Castañeda 2009 (H)

Mycocentrodochium K. Matsush. \& Mat-sush. 1996 (H)

Mycoënterolobium Goos 1970 (H)

Mycomyces Wyss-Chod. 1928 (H)

Mycopara Bat. \& J.L. Bezerra 1960 (C)

Mycospraguea U. Braun \& Rogerson 1993 (C)

Mycosticta Höhn. 1918 (C)

Mycosylva M.C. Tulloch 1973 (H)

Mycousteria M.L. Farr 1986 (C)

Myiocoprula Petr. 1955 (C)

Myriellina Höhn. 1915 (C)

Myrmecomyces Jouvenaz \& Kimbr. 1991 (H)

Myrotheciastrum Abbas \& B. Sutton 1988 (H)

Mystrosporiella Munjal \& Kulshr. 1969 (H)

Myxoparaphysella Caball. 1941 (C)

Myxosporella Sacc. 1881 (C)

Myxosporidiella Negru 1960 (C)

Myxostomellina Syd. 1931 (C)

Myxothyriopsis Bat. \& A.F. Vital 1956 (C)

Myxothyrium Bubák \& Kabát 1915 (C)

Naemosphaera P. Karst. 1888 (C)

Naemosphaerella Höhn. 1923 (C)

Nagrajia R.F. Castañeda \& W.B. Kendr. 1991 (C)

Nagrajomyces Mel'nik 1984 (C)

Nakatopsis Whitton et al. 2001 (H)

Nanoschema B. Sutton 1980 (C)

Naothyrsium Bat. 1960 (C)

Necraphidium Cif. 1951 (H)

Nematogonum Desm. 1834 (H)

Nematographium Goid. 1935 (H)

Nemozythiella Höhn. 1925 (C)

Neoalpakesa Punith. 1981 (C)

Neoarbuscula B. Sutton 1983 (H)

Neobarclaya Sacc. 1899 (C)

Neodiplodina Petr. 1954 (C)

Neofuckelia Zeller \& Goodd. 1935 (C)

Neoheteroceras Nag Raj 1993 (C)

Neojohnstonia B. Sutton 1983 (H)

Neoligniella Naumov 1951 (C)

Neomarssoniella U. Braun 1991 (C)

Neopeltis Syd. 1937 (C)

Neopericonia Kamal et al. 1983 (H) 
Neophoma Petr. \& Syd. 1927 (C)

Neoplaconema B. Sutton 1977 (C)

Neopodoconis Rifai 2008 (H)

Neospegazzinia Petr. \& Syd. 1936 (C)

Neottiospora Desm. 1843 (C)

Neozythia Petr. 1958 (C)

Neta Shearer \& J.L. Crane 1971 (H)

Nidulispora Nawawi \& Kuthub. 1990 (H)

Nigrolentilocus R.F. Castañeda et al. 2001 (H)

Nigromacula Etayo 2002 (H)

Nigropuncta D. Hawksw. 1981 (C)

Nosophloea Fr. 1849 (C)

Nothospora Peyronel 1913 (H)

Novozymia W.P. Wu 2005 (H)

Nummospora E. Müll. \& Shoemaker 1964 (C)

Nusia Subram. 1995 (H)

Nyctalospora E.F. Morris 1972 (H)

Nypaella K.D. Hyde \& B. Sutton 1992 (C)

Obeliospora Nawawi \& Kuthub. 1990 (H)

Obstipipilus B. Sutton 1968 (C)

Octopodotus Kohlm. \& Volkm.-Kohlm. 2003 (C)

Odontodictyospora Mercado 1984 (H)

Oedothea Syd. 1930 (H)

Ojibwaya B. Sutton 1973 (H)

Olpitrichum G.F. Atk. 1894 (H)

Omega B. Sutton \& Minter 1988 (C)

Oncopodium Sacc. 1904 (H)

Oncospora Kalchbr. 1880 (C)

Oncosporella P. Karst. 1887 (C)

Oncostroma Bat. \& Marasas 1966 (C)

Onychophora W. Gams et al. 1984 (H)

Oosporidium Stautz 1931 (Y)

Oothyrium Syd. 1939 (C)

Ophiosira Petr. 1955 (C)

Orbimyces Linder 1944 (H)

Orphanocoela Nag Raj 1989 (C)

Ostracoderma Fr. 1825 (C)

Ostracodermidium Mukerji 1973 (H)

Oswaldina Rangel 1921 (C)

Ovadendron Sigler \& J.W. Carmich. 1976 (H)

Paathramaya Subram. 1956 (H)

Pachycladina Marvanová 1987 (H)

Palawaniopsis Bat. et al. 1959 (C)

Paliphora Sivan. \& B. Sutton 1985 (H)

Papilionospora V.G. Rao \& B. Sutton 1976 (H)

Pappimyces B. Sutton \& Hodges 1975 (H)

Paraaoria R.K. Verma \& Kamal 1987 (C)

Paraarthrocladium Matsush. 1993 (H)

Parablastocatena Y.D. Zhang \& X.G. Zhang 2012 (H)

Paraceratocladium R.F. Castañeda 1987 (H)

Parachionomyces Thaung 1979 (H)

Paracostantinella Subram. \& Sudha 1989 (H) 
Paracryptophiale Kuthub. \& Nawawi 1994 (H)

Paracytospora Petr. 1925 (C)

Paradendryphiopsis M.B. Ellis 1976 (H)

Paradidymobotryum C.J.K. Wang \& B. Sutton 1984 (H)

Paradiplodia Speg. ex Trotter 1931 (C)

Paradischloridium Bhat \& B. Sutton 1985 (H)

Paradiscula Petr. 1941 (C)

Paraëpicoccum Matsush. 1993 (H)

Parafulvia Kamal et al. 1983 (H)

Parahaplotrichum W.A. Baker \& Partr. 2001 (H)

Paraharknessia Matsush. 2003 (H)

Parahyalotiopsis Nag Raj 1976 (C)

Paramassariothea Subram. \& Muthumary 1979 (C)

Paramenisporopsis Matsush. 2003 (H)

Parapericonia M.B. Ellis 1976 (H)

Parapericoniella U. Braun et al. 2005 (H)

Paraphaeoisaria de Hoog \& Morgan-Jones 1978 (H)

Parapithomyces Thaung 1976 (H)

Parapyricularia M.B. Ellis 1972 (H)

Pararobillarda Matsush. 1996 (C)

Parasphaeropsis Petr. 1953 (C)

Parastenella J.C. David 1991 (H)

Parastigmatellina Bat. \& C.A.A. Costa 1959 (C)

Paratetraploa M.K.M. Wong \& K.D. Hyde 2002 (H)

Paratomenticola M.B. Ellis 1976 (H)

Paratrichoconis Deighton \& Piroz. 1972 (H)

Paraulocladium R.F. Castañeda 1986 (H)

Paspalomyces Linder 1933 (H)

Patriciomyces D. Hawksw. 2001 (H)

Pazschkeella Syd. \& P. Syd. 1901 (C)

Peethasthabeeja P. Rag. Rao 1981 (H)

Pellionella (Sacc.) Sacc. 1902 (C)

Peltasterinostroma Punith. 1975 (C)

Peltasteropsis Bat. \& H. Maia 1959 (C)

Peltistroma Henn. 1904 (C)

Peltistromella Höhn. 1907 (C)

Peltosoma Syd. 1925 (C)

Peltostromellina Bat. \& A.F. Vital 1959 (C)

Peltostromopsis Bat. \& A.F. Vital 1959 (C)

Penzigomyces Subram. 1992 (H)

Perelegamyces R.F. Castañeda \& W.B. Kendr. 1990 (H)

Perizomella Syd. 1927 (C)

Pestalozziella Sacc. \& Ellis ex Sacc. 1882 (C)

Petrakiopsis Subram. \& K.R.C. Reddy 1968 (H)

Phacostroma Petr. 1955 (C)

Phacostromella Petr. 1955 (C)

Phaeoblastophora Partr. \& Morgan-Jones 2002 (H)

Phaeocandelabrum R.F. Castañeda et al. 2009 (H)

Phaeodactylium Agnihothr. 1968 (H)

Phaeodiscula Cub. 1891 (C)

Phaeodomus Höhn. 1909 (C)

Phaeohiratsukaea Udagawa \& Iwatsu 1990 (H) 
Phaeoidiomyces Dorn.-Silva \& Dianese 2004 (H)

Phaeolabrella Speg. 1912 (C)

Phaeomonilia R.F. Castañeda et al. 2007 (H)

Phaeomonostichella Keissl. ex Petr. 1941 (C)

Phaeophloeosporella Crous \& B. Sutton 1997 (C)

Phaeophomopsis Höhn. 1917 (C)

Phaeoschizotrichum Silva et al. 2015 (H)

Phaeosporobolus D. Hawksw. \& Hafellner 1986 (H)

Phaeostalagmus W. Gams 1976 (H)

Phaeothyrium Petr. 1947 (C)

Phaeotrichoconis Subram. 1956 (H)

Phaeoxyphiella Bat. \& Cif. 1963 (C)

Phanerococculus Cif. 1954 (C)

Phialoarthrobotryum Matsush. 1975 (H)

Phialogeniculata Matsush. 1971 (H)

Phialophaeoisaria Matsush. 1995 (H)

Phialosporostilbe Mercado \& J. Mena 1985 (H)

Phialostele Deighton 1969 (H)

Phialotubus R.Y. Roy \& Leelav. 1966 (H)

Phloeoconis Fr. 1849 (H)

Phloeosporina Höhn. 1924 (C)

Phlyctaeniella Petr. 1922 (C)

Phomachora Petr. \& Syd. 1925 (C)

Phomachorella Petr. 1947 (C)

Phomatosporella Tak. Kobay. \& K. Sasaki 1982 (C)

Phomyces Clem. 1931 (C)

Phragmoconidium G.F. Sepúlveda et al. 2009 (H)

Phragmopeltis Henn. 1904 (C)

Phragmospathula Subram. \& N.G. Nair 1966 (H)

Phragmospathulella J. Mena \& Mercado 1986 (H)

Phylloedium Fr. 1825 (H)

Phyllohendersonia Tassi 1902 (C)

Physalidiella Rulamort 1990 (H)

Physalidiopsis R.F. Castañeda \& W.B. Kendr. 1990 (H)

Piggotia Berk. \& Broome 1851 (C)

Pinatubo J.B. Manandhar \& Mew 1996 (H)

Piperivora Siboe et al. 1999 (H)

Piricaudilium Hol.-Jech. 1988 (H)

Piricaudiopsis J. Mena \& Mercado 1987 (H)

Pirispora Faurel \& Schotter 1966 (C)

Pirostomella Sacc. 1914 (H)

Pirozynskia Subram. 1972 (H)

Pithosira Petr. 1949 (H)

Pittostroma Kowalski \& T.N. Sieber 1992 (C)

Placella Syd. 1938 (C)

Placodiplodia Bubák 1916 (C)

Placomelan Cif. 1962 (C)

Placonema (Sacc.) Petr. 1921 (C)

Placonemina Petr. 1921 (C)

Placosphaerina Maire 1917 (C)

Placothea Syd. 1931 (C)

Placothyrium Bubák 1916 (C) 
Plagiostigmella Petr. 1949 (C)

Plasia Sherwood 1981 (C)

Plectonaemella Höhn. 1915 (C)

Plectopeltis Syd. 1927 (C)

Plectophomopsis Petr. 1922 (C)

Plectopycnis Bat. \& A.F. Vital 1959 (C)

Plectosira Petr. 1929 (C)

Plectronidiopsis Nag Raj 1979 (C)

Plectronidium Nag Raj 1977 (C)

Plenocatenulis Bat. \& Cif. 1959 (C)

Plenophysa Syd. \& P. Syd. 1920 (C)

Plenotrichaius Bat. \& Valle 1961 (C)

Plenotrichopsis Bat. 1961 (C)

Plenotrichum Syd. 1927 (C)

Plenozythia Syd. \& P. Syd. 1916 (C)

Pleocouturea G. Arnaud 1911 (C)

Plesiospora Drechsler 1971 (H)

Pleurocolla Petr. 1924 (H)

Pleurodesmospora Samson et al. 1979 (H)

Pleurodiscula Höhn. 1926 (C)

Pleurodomus Petr. 1934 (C)

Pleuropedium Marvanová \& S.H. Iqbal 1973 (H)

Pleurophomopsis Petr. 1924 (C)

Pleuroplaconema Petr. 1923 (C)

Pleuroplacosphaeria Syd. 1928 (C)

Pleurostromella Petr. 1922 (C)

Pleurotheciopsis B. Sutton 1973 (H)

Pleurothyriella Petr. \& Syd. 1925 (C)

Pleurovularia R. Kirschner \& U. Braun 2002 (H)

Pocillopycnis Dyko \& B. Sutton 1979 (C)

Podoplaconema Petr. 1921 (C)

Podosporiella Ellis \& Everh. 1894 (H)

Podosporiopsis Jian Ma et al. 2016 (H)

Podosporium Schwein. 1832 (H)

Poikilosperma Bat. \& J.L. Bezerra 1961 (C)

Polybulbophiale Goh \& K.D. Hyde 1998 (H)

Polychaetella Speg. 1918 (C)

Polycladium Ingold 1959 (H)

Polydesmus Mont. 1845 (H)

Polyetron Bat. \& Peres 1963 (C)

Polylobatispora Matsush. 1996 (H)

Polypaecilum G. Sm. 1961 (H)

Polyrostrata T.P. Devi \& N. Mathur 2009 (C)

Polystomellomyces Bat. 1959 (C)

Polystratorictus Matsush. 1993 (H)

Polysynnema Constant. \& Seifert 1988 (H)

Polytretophora Mercado 1983 (H)

Porocladium Descals 1976 (H)

Poropeltis Henn. 1904 (C)

Porophilomyces U. Braun 2000 (H)

Porosubramaniania Hol.-Jech. 1985 (H)

Porrectotheca Matsush. 1996 (C) 
Prathoda Subram. 1956 (H)

Proboscispora Punith. 1984 (C)

Prosthemiella Sacc. 1881 (H)

Protostegiomyces Bat. \& A.F. Vital 1955 (C)

Protostroma Bat. 1957 (C)

Psammina Sacc. \& M. Rousseau ex E. Bommer \& M. Rousseau 1891 (C)

Pseudoacrodictys W.A. Baker \& Morgan-Jones 2003 (H)

Pseudoanguillospora S.H. Iqbal 1974 (H)

Pseudoaristastoma Suj. Singh 1979 (C)

Pseudoasperisporium U. Braun 2000 (H)

Pseudobasidiospora Dyko \& B. Sutton 1978 (C)

Pseudocanalisporium R.F. Castañeda \& W.B. Kendr. 1991 (H)

Pseudocenangium P. Karst. 1886 (C)

Pseudochuppia Kamal et al. 1984 (H)

Pseudoclathrosphaerina Voglmayr 1997 (H)

Pseudoconium Petr. 1969 (C)

Pseudocytoplacosphaeria Punith. \& Spooner 2002 (C)

Pseudocytospora Petr. 1923 (C)

Pseudodichomera Höhn. 1918 (C)

Pseudodidymaria U. Braun 1993 (H)

Pseudodiplodia (P. Karst.) Sacc. 1884 (C)

Pseudodiscula Laubert 1911 (C)

Pseudofuscophialis Sivan. \& H.S. Chang 1995 (H)

Pseudogaster Höhn. 1907 (H)

Pseudographiella E.F. Morris 1966 (H)

Pseudomeria G.L. Barron 1980 (H)

Pseudomicrodochium B. Sutton 1975 (H)

Pseudoneottiospora Faurel \& Schotter 1965 (C)

Pseudopatellina Höhn. 1908 (C)

Pseudopeltistroma Katum. 1975 (C)

Pseudopetrakia M.B. Ellis 1971 (H)

Pseudophloeosporella U. Braun 1993 (C)

Pseudophragmotrichum W.P. Wu et al. 1998 (C)

Pseudopolystigmina Murashk. 1928 (C)

Pseudoramularia Matsush. 1983 (H)

Pseudorhizopogon Kobayasi 1983 (C)

Pseudoschizothyra Punith. 1980 (C)

Pseudosigmoidea K. Ando \& N. Nakam. 2000 (H)

Pseudostegia Bubák 1906 (C)

Pseudothyrium Höhn. 1927 (C)

Pseudotorula Subram. 1958 (H)

Pseudotracylla B. Sutton \& Hodges 1976 (C)

Pseudotrichoconis W.A. Baker \& Morgan-Jones 2001 (H)

Pseudozythia Höhn. 1902 (C)

Pterulopsis Wakef. \& Hansf. 1943 (H)

Pterygosporopsis P.M. Kirk 1983 (H)

Pucciniospora Speg. 1886 (C)

Pulchromyces Hennebert 1973 (H)

Pullospora Faurel \& Schotter 1965 (C)

Pulvinella A.W. Ramaley 2001 (H)

Punctillina Toro 1934 (C)

Pycnidioarxiella Punith. \& N.D. Sharma 1980 (C) 
Pycnidiopeltis Bat. \& C.A.A. Costa 1959 (C)

Pycnis Bref. 1881 (C)

Pycnodactylus Bat. et al. 1967 (C)

Pycnodallia Kohlm. \& Volkm.-Kohlm. 2001 (C)

Pycnoharknessia Matsush. 1996 (C)

Pycnomma Syd. 1924 (C)

Pycnomoreletia Rulamort 1990 (C)

Pycnoseynesia Kuntze 1898 (C)

Pycnothera N.D. Sharma \& G.P. Agarwal 1974 (C)

Pycnothyriella Bat. 1952 (C)

Pycnothyrium Died. 1913 (C)

Pyramidospora Sv. Nilsson 1962 (H)

Pyrenyllium Clem. 1909 (C)

Pyrgostroma Petr. 1951 (C)

Pyripnomyces Cavalc. 1972 (C)

Quadracaea Lunghini et al. 1996 (H)

Quadricladium Nawawi \& Kuthub. 1989 (H)

Quasidiscus B. Sutton 1991 (C)

Quasiphloeospora B. Sutton et al. 1996 (H)

Queenslandia Bat. \& H. Maia 1959 (C)

Quezelia Faurel \& Schotter 1965 (C)

Radiatispora Matsush. 1995 (H)

Raizadenia S.L. Srivast. 1981 (H)

Ramakrishnanella Kamat \& Ullasa ex Ullasa 1970 (C)

Ramicapitulum Whitton et al. 2012 (H)

Ramicephala Voglmayr \& G. Delgado 2003 (H)

Ramiphialis F.R. Barbosa et al. 2020 (H)

Ramoconidiifera B. Sutton et al. 1996 (H)

Redbia Deighton \& Piroz. 1972 (H)

Refractohilum D. Hawksw. 1977 (H)

Repetoblastiella R.F. Castañeda et al. 2010 (H)

Retroconis de Hoog \& Bat. Vegte 1989 (H)

Rhabdoclema Syd. 1939 (C)

Rhabdogloeopsis Petr. 1925 (C)

Rhabdostromella Höhn. 1915 (C)

Rhabdostromina Died. 1921 (C)

Rhexoacrodictys W.A. Baker \& Morgan-Jones 2002 (H)

Rhexoampullifera P.M. Kirk 1982 (H)

Rhexoprolifer Matsush. 1996 (H)

Rhinotrichella G. Arnaud ex de Hoog 1977 (H)

Rhipidocephalum Trail 1888 (H)

Rhizosphaerina B. Sutton 1986 (C)

Rhodesia Grove 1937 (C)

Rhodesiopsis B. Sutton \& R. Campb. 1979 (C)

Rhodosticta Woron. 1911 (C)

Rhodothallus Bat. \& Cif. 1959 (H)

Rhombostilbella Zimm. 1902 (H)

Rhopalocladium Schroers et al. 1999 (H)

Rhynchodiplodia Briosi \& Farneti 1906 (C)

Rhynchomyces Willk. 1866 (H)

Rhynchoseptoria Unamuno 1940 (C)

Rhynchosporina Arx 1957 (H) 
Riclaretia Peyronel 1915 (H)

Rileya A. Funk 1979 (C)

Robakia Petr. 1952 (C)

Rogergoosiella A. Hern. et al. 1996 (H)

Roscoepoundia Kuntze 1898 (C)

Rosulomyces S. Marchand \& Cabral 1976 (H)

Rota Bat. et al. 1959 (C)

Ruggieria Cif. \& Montemart. 1958 (C)

Sadasivania Subram. 1957 (H)

Sanjuanomyces R.F. Castañeda \& W.B. Kendr. 1991 (H)

Santapauinda Subram. 1995 (H)

Saprophragma K.B. Deshp. \& K.S. Deshp. 1966 (H)

Sarcinosporon D.S. King \& S.C. Jong 1975 (H)

Sarcophoma Höhn. 1916 (C)

Sarophorum Syd. \& P. Syd. 1916 (H)

Sativumoides S.C. Ren et al. 2012 (H)

Scaphidium Clem. 1901 (C)

Scenomyces F. Stevens 1927 (H)

Sceptrifera Deighton 1965 (H)

Schizothyra Bat. \& C.A.A. Costa 1957 (C)

Schizothyrella Thüm. 1880 (C)

Schizothyropsis Bat. \& A.F. Vital 1960 (C)

Schizotrichum McAlpine 1903 (H)

Schroeteria G. Winter 1881 (H)

Schwarzmannia Pisareva 1968 (C)

Scirrhophoma Petr. 1941 (C)

Sclerococcum Fr. 1825 (H)

Sclerographiopsis Deighton 1973 (H)

Sclerographium Berk. 1854 (H)

Scleromeris Syd. 1926 (C)

Sclerophoma Höhn. 1909 (C)

Scleropycnis Syd. \& P. Syd. 1911 (C)

Sclerotiella A.K. Sarbhoy \& A. Sarbhoy 1975 (H)

Sclerozythia Petch 1937 (C)

Scolecobasidiella M.B. Ellis 1971 (H)

Scolecobeltrania Iturr. et al. 2013 (H)

Scolecodochium K. Matsush. \& Matsush. 1996 (H)

Scolecosporiella Petr. 1921 (C)

Scolecotheca Søchting \& B. Sutton 1997 (C)

Scolecozythia Curzi 1927 (C)

Scoliotidium Bat. \& Cavalc. 1963 (C)

Scopaphoma Dearn. \& House 1925 (C)

Scopulariella Gjaerum 1971 (H)

Scothelius Bat. et al. 1965 (C)

Scutisporus K. Ando \& Tubaki 1985 (H)

Scutopeltis Bat. \& H. Maia 1957 (C)

Scutopycnis Bat. 1957 (C)

Seimatosporiopsis B. Sutton et al. 1972 (C)

Selenosira Petr. 1957 (C)

Selenosporopsis R.F. Castañeda \& W.B. Kendr. 1991 (H)

Semipseudocercospora J.M. Yen 1983 (H)

Septocytella Syd. 1929 (C) 
Septogloeum Sacc. 1880 (C)

Septoidium G. Arnaud 1921 (H)

Septomyxella (Höhn.) Höhn. 1923 (C)

Septopatella Petr. 1925 (C)

Septosporiopsis W.A. Baker \& Morgan-Jones 2009 (H)

Septosporium Corda 1831 (H)

Septotrullula Höhn. 1902 (H)

Sessiliospora D. Hawksw. 1979 (H)

Setolibertella Punith. \& Spooner 1999 (C)

Setophiale Matsush. 1995 (H)

Setosporella Mustafa \& Abdul-Wahid 1989 (H)

Seychellomyces Matsush. 1981 (H)

Seynesiopsis Henn. 1904 (C)

Shawiella Hansf. 1957 (C)

Sheariella Petr. 1952 (C)

Sheathnema Dubey \& Moonambeth 2014 (H)*

Shivomyces Hosag. 2004 (H)

Siamia V. Robert et al. 2000 (H)

Sigmatomyces Sacc. \& P. Syd. 1913 (H)

Simmonsiella J.L. Crane \& A.N. Mill. 2016 (H)

Sirexcipula Bubák 1907 (C)

Sirocyphis Clem. 1909 (C)

Sirodochiella Höhn. 1925 (H)

Sirogloea Petr. 1923 (C)

Siroligniella Naumov 1926 (C)

Sirophoma Höhn. 1917 (C)

Siroplacodium Petr. 1940 (C)

Siropleura Petr. 1934 (C)

Siroscyphellina Petr. 1923 (C)

Sirosperma Syd. \& P. Syd. 1916 (C)

Sirosphaera Syd. \& P. Syd. 1913 (C)

Sirosporonaemella Naumov 1951 (C)

Sirothecium P. Karst. 1887 (C)

Sirothyriella Höhn. 1910 (C)

Sirothyrium Syd. \& P. Syd. 1916 (C)

Sirozythia Höhn. 1904 (C)

Sirozythiella Höhn. 1909 (C)

Sitochora H.B.P. Upadhyay 1964 (C)

Slimacomyces Minter 1986 (H)

Soloacrospora W.B. Kendr. \& R.F. Castañeda 1991 (H)

Solosympodiella Matsush. 1971 (H)

Soloterminospora Matsush. 1996 (H)

Spermatoloncha Speg. 1908 (H)

Spermatoloncha Speg. 1908 (H)

Spermochaetella Cif. 1954 (C)

Spermospora R. Sprague 1948 (H)

Spermosporella Deighton 1969 (H)

Sphaeridium Fresen. 1852 (H)

Sphaeriostromella Bubák 1916 (C)

Sphaeriothyrium Bubák 1916 (C)

Sphaeromma H.B.P. Upadhyay 1964 (C)

Sphaeronema Fr. 1815 (C) 
Sphaerophoma Petr. 1924 (C)

Sphaerulomyces Marvanová 1977 (H)

Sphondylocephalum Stalpers 1974 (H)

Spicularia Pers. 1822 (H)

Spilodochium Syd. 1927 (H)

Spinulospora Deighton 1973 (H)

Spiralum J.L. Mulder 1975 (H)

Spiropes Cif. 1955 (H)

Spondylocladiella Linder 1934 (H)

Spondylocladiopsis M.B. Ellis 1963 (H)

Sporhaplus H.B.P. Upadhyay 1964 (C)

Sporidesmiopsis Subram. \& Bhat 1989 (H)

Sporoglena Sacc. 1894 (H)

Sporophiala P.Rag. Rao 1970 (H)

Sporophora Luteraan 1952 (H)

Stachybotryella Ellis \& Barthol. 1902 (H)

Stachybotryna Tubaki \& T. Yokoy. 1971 (H)

Stagonopatella Petr. 1927 (C)

Stagonopsis Sacc. 1884 (C)

Stagonosporina Tassi 1902 (C)

Stagonostromella Petr. \& Syd. 1927 (C)

Staheliella Emden 1974 (H)

Stalagmochaetia Cif. \& Bat. 1963 (C)

Stanhughesiella R.F. Castañeda \& D.W. Li 2017 (H)

Stauronema (Sacc.) Syd. et al. 1916 (C)

Stauronematopsis Abbas et al. 2002 (C)

Staurophoma Höhn. 1907 (C)

Stegonsporiopsis Van Warmelo \& B. Sutton 1981 (C)

Stellomyces Morgan-Jones et al. 1987 (H)

Stellopeltis Bat. \& A.F. Vital 1959 (C)

Stellospora Alcorn \& B. Sutton 1984 (H)

Stellothyriella Bat. \& Cif. 1959 (C)

Stemphyliomma Sacc. \& Traverso 1911 (H)

Stenellopsis B. Huguenin 1966 (H)

Stenocephalopsis Chamuris \& C.J.K. Wang 1998 (H)

Stenocladiella Marvanová \& Descals 1987 (H)

Stenospora Deighton 1969 (H)

Stephembruneria R.F. Castañeda 1988 (H)

Stevensonula Petr. 1952 (C)

Stichospora Petr. 1927 (C)

Stictopatella Höhn. 1918 (C)

Stictosepta Petr. 1964 (C)

Stigmatellina Bat. \& H. Maia 1960 (C)

Stigmella Lév. 1842 (C)

Stigmina Sacc. 1980 (H)

Stigmopeltis Syd. 1927 (C)

Stilbellula Boedijn 1951 (H)

Stilbodendron Syd. \& P. Syd. 1916 (H)

Stilbophoma Petr. 1942 (C)

Stilbothamnium Henn. 1896 (H)

Strasseria Bres. \& Sacc. 1902 (C)

Strasseriopsis B. Sutton \& Tak. Kobay. 1970 (C) 
Stratiphoromyces Goh \& K.D. Hyde 1998 (H)

Striosphaeropsis Verkley \& Aa 1997 (C)

Stromatocrea W.B. Cooke 1952 (H)

Stromatopogon Zahlbr. 1897 (C)

Stromatopycnis A.F. Vital 1956 (C)

Stromatostysanus Höhn. 1919 (H)

Strongylothallus Bat. \& Cif. 1959 (H)

Stygiomyces Coppins \& S.Y. Kondr. 1995 (C)

Stylaspergillus B. Sutton et al. 1982 (H)

Subhysteropycnis Wedin \& Hafellner 1998 (C)

Subicularium M.L. Farr \& Goos 1989 (H)

Subramania D. Rao \& P.Rag. Rao 1964 (H)

Subramanianospora Narayanan et al. 2003 (H)

Subulispora Tubaki 1971 (H)

Suttoniella S. Ahmad 1961(C)

Suttonina H.C. Evans 1984 (C)

Syamithabeeja Subram. \& Natarajan 1976 (H)

Sylviacollaea Cif. 1963 (C)

Symphysos Bat. \& Cavalc. 1967 (C)

Sympodiella W.B. Kendr. 1958 (H)

Sympodiocladium Descals 1982 (H)

Sympodioclathra Voglmayr 1997 (H)

Sympodioplanus R.C. Sinclair \& Boshoff 1997 (H)

Sympodiosynnema J.W. Xia \& X.G. Zhang 2016 (H)

Synchronoblastia Uecker \& F.L. Caruso 1988 (C)

Syncladium Rabenh. 1859 (C)

Synnemacrodictys W.A. Baker \& Morgan-Jones 2009 (H)

Synnemaseimatoides K. Matsush. \& Matsush. 1996 (H)

Synnematium Speare 1920 (H)

Synnematomyces Kobayasi 1981 (H)

Synostomina Petr. 1949 (C)

Systremmopsis Petr. 1923 (H)

Taeniolina M.B. Ellis 1976 (H)

Talpapellis Alstrup \& M.S. Cole 1998 (H)

Tandonea M.D. Mehrotra 1991 (C)

Tarsodisporus Bat. \& A.A. Silva 1965 (C)

Tandonella S.S. Prasad \& R.A.B. Verma 1970 (H)

Tectacervulus A.W. Ramaley 1992 (C)

Telligia Hendr. 1948 (H)

Temerariomyces B. Sutton 1993 (H)

Teratosperma Syd. \& P. Syd. 1909 (H)

Termitaria Thaxt. 1920 (H)

Termitariopsis M. Blackw et al. 1980 (H)

Tetrabrachium Nawawi \& Kuthub. 1987 (H)

Tetrabrunneospora Dyko 1978 (H)

Tetracoccosporium Szabó 1905 (H)

Tetracrium Henn. 1902 (H)

Tetrameronycha Speg. ex W. Rossi \& M. Blackw. 1990 (H)

Tetranacriella Kohlm. \& Volkm.-Kohlm. 2001 (C)

Tetranacrium H.J. Huds. \& B. Sutton 1964 (C)

Tetraposporium S. Hughes 1951 (H)

Textotheca Matsush. 1996 (C) 
Thallospora L.S. Olive 1948 (H)

Thaptospora B. Sutton \& Pascoe 1987 (C)

Tharoopama Subram. 1956 (H)

Thirumalacharia Rathaiah 1981 (H)

Tholomyces Matsush. 2003 (C)

Thoracella Oudem. 1900 (C)

Thrinacospora Petr. 1948 (C)

Thyriostromella Bat. \& C.A.A. Costa 1959 (C)

Thyrostromella Höhn. 1919 (H)

Thyrsidiella Höhn. ex Höhn. 1909 (C)

Thyrsidina Höhn. 1905 (C)

Tiarosporellivora Punith. 1981 (C)

Ticogloea G. Weber et al. 1994 (H)

Ticosynnema R.F. Castañeda et al. 2013 (H)*

Tilakiopsis V.G. Rao 1994 (H)

Titaea Sacc. 1876 (H)

Titaeopsis B. Sutton \& Deighton 1984 (H)

Titaeospora Bubák 1916 (C)

Tomenticola Deighton 1969 (H)

Tompetchia Subram. 1985 (H)

Torulopsiella Bender 1932 (H)

Toxosporiella B. Sutton 1986 (C)

Toxosporiopsis B. Sutton \& Sellar 1966 (C)

Toxosporium Vuill. 1896 (H)

Trematophoma Petr. 1924 (C)

Tremellidium Petr. 1927 (C)

Tretendophragmia Subram. 1995 (H)

Tretocephala Subram. 1995 (H)

Tretolylea Cantillo et al. 2015 (H)

Tretophragmia Subram. \& Natarajan 1974 (H)

Tretospeira Piroz. 1972 (H)

Tretospora M.B. Ellis 1976 (H)

Tretovularia Deighton 1984 (H)

Tribolospora D.A. Reid 1966 (C)

Tricellula Beverw. 1954 (H)

Trichaegum Corda 1837 (H)

Trichobolbus Bat. 1964 (C)

Trichobotrys Penz. \& Sacc. 1901 (H)

Trichoconiella B.L. Jain 1976 (H)

Trichoconis Clem. 1909 (H)

Trichodiscula Vouaux 1910 (C)

Trichodochium Syd. 1927 (H)

Trichomatoclava G.F. Sepúlveda et al. 2009 (H)

Trichomatomyces Dorn.-Silva \& Dianese 2004 (H)

Trichomatosphaera Pereira-Carv. et al. 2009 (H)

Trichopeltulum Speg. 1889 (C)

Trichoseptoria Cavara 1892 (C)

Trichosporiella Kamyschko 1960 (H)

Trichosporodochium Dorn.-Silva \& Dia-nese 2004 (H)

Trichotheca P. Karst. 1887 (H)

Tricladiella K. Ando \& Tubaki 1984 (H)

Tricladiopsis Descals 1982 (H) 
Tricladiospora Nawawi \& Kuthub. 1988 (H)

Tricornispora Bonar 1967 (H)

Trifurcospora K. Ando \& Tubaki 1988 (H)

Triglyphium Fresen. 1852 (H)

Trigonosporium Tassi 1900 (C)

Tripoconidium Subram. 1978 (H)

Triposporina Höhn. 1912 (H)

Triramulispora Matsush. 1975 (H)

Triscelophorus Ingold 1944 (H)

Triscelosporium Nawawi \& Kuthub. 1987 (H)

Trisulcosporium H.J. Huds. \& B. Sutton 1964 (H)

Troposporium Harkn. 1884 (H)

Troposporopsis Whitton et al. 1999 (H)

Tryblidiopycnis Höhn. 1918 (C)

Tryssglobulus B. Sutton \& Pascoe 1987 (H)

Tuberculariopsis Höhn. 1909 (H)

Tuberculispora Deighton \& Piroz. 1972 (H)

Tulipispora Révay \& Gönczöl 2009 (H)

Tunicago B. Sutton \& Pollack 1977 (C)

Turturconchata J.L. Chen et al. 1999 (H)

Tympanosporium W. Gams 1974 (H)

Uberispora Piroz. \& Hodges 1973 (H)

Ubrizsya Negru 1965 (C)

Ulocoryphus Michaelides et al. 1982 (H)

Umbellidion B. Sutton \& Hodges 1975 (H)

Uniseta Ciccar. 1948 (C)

Urohendersonia Speg. 1902 (C)

Urohendersoniella Petr. 1955 (C)

Uvarispora Goos \& Piroz. 1975 (H)

Vagnia D. Hawksw. \& Miądl. 1997 (C)

Vanakripa Bhat et al. 1993 (H)

Vanbeverwijkia Agnihothr. 1961 (H)

Vanderystiella Henn. 1908 (C)

Vanterpoolia A. Funk 1982 (H)

Vasudevella Chona et al. 1957 (C)

Velutipila D. Hawksw. 1987 (H)

Ventrographium H.P. Upadhyay et al. 1986 (H)

Venustocephala Matsush. 1995(H)

Venustosynnema R.F. Castañeda \& W.B. Kendr. 1990 (H)

Veracruzomyces Mercado et al. 2002 (H)

Veramycella G. Delgado 2009 (H)

Veramyces Matsush. 1993 (H)

Verdipulvinus A.W. Ramaley 1999 (H)

Veronaella Subram. \& K.R.C. Reddy 1975 (C)

Veronidia Negru 1964 (C)

Verrucariella S. Ahmad 1967 (C)

Verrucophragmia Crous et al. 1994 (H)

Verticicladus Matsush. 1993 (H)

Vesicladiella Crous \& M.J. Wingf. 1994 (H)

Vesiculohyphomyces Armando et al. 2009 (H)

Vestigium Piroz. \& Shoemaker 1972 (C)

Virgariella S. Hughes 1953 (H) 
Viscomacula R. Sprague 1951 (H)

Vittalia Gaws \& Bhat $2007(\mathbf{H})$

Vizellopsidites M.A. Khan et al. 2018 (C)

Vouauxiella Petr. \& Syd. 1927 (C)

Waihonghopes Yanna \& K.D. Hyde 2002 (H)

Wardinella Bat. \& Peres 1960 (C)

Websteromyces W.A. Baker \& Partr. 2000 (H)

Weissia Bat. \& M.P. Herrera 1964 (C)

Weufia Bhat \& B. Sutton 1985 (H)

Wojnowicia Sacc. 1899 (C)

Xenidiocercus Nag Raj 1993 (C)

Xenochora Petr. 1948 (C)

Xenodomus Petr. 1922 (C)

Xenoheteroconium Bhat et al. 1993 (H)

Xenokylindria DiCosmo et al. 1983 (H)

Xenopeltis Syd. \& P. Syd. 1919 (C)

Xenoplaca Petr. 1949 (H)

Xenostroma Höhn. 1915 (C)

Xeroconium D. Hawksw. 1981 (C)

Xiambola Minter \& Hol.-Jech. 1981 (H)

Xiphomyces Syd. \& P. Syd. 1916 (H)

Xiuguozhangia K. Zhang et al. 2014 (H)

Xylochia B. Sutton 1983 (H)

Xyloglyphis Clem. 1909 (C)

Xylohypha (Fr.) E.W. Mason 1960 (H)

Xylohyphopsis W.A. Baker \& Partr. 2000 (H)

Yalomyces Nag Raj 1993 (C)

Yinmingella Goh et al. 1999 (H)

Yoshinagaia Henn. 1904 (C)

Ypsilomyces D.A.C. Almeida \& Gusmão 2014 (H)*

Yuccamyces Gour et al. 1979 (H)

Zakatoshia B. Sutton 1973 (H)

Zebrospora McKenzie 1991 (H)

Zelandiocoela Nag Raj 1993 (C)

Zelodactylaria A.C. Cruz et al. 2012 (H)

Zelopelta B. Sutton \& R.D. Gaur 1984 (C)

Zelosatchmopsis Nag Raj 1991 (C)

Zelotriadelphia R.F. Castañeda et al. 2005 (H)

Zetesimomyces Nag Raj 1988 (C)

Zevadia J.C. David \& D. Hawksw. 1995 (H)

Zilingia Petr. 1934 (C)

Zinzipegasa Nag Raj 1993 (C)

Zopheromyces B. Sutton \& Hodges 1977 (H)

Zunura Nag Raj 1993 (C)

Zygosporium Mont. 1842 (H)

Zythia Fr. 1849 (C)

Zyxiphora B. Sutton 1981 (H)

Phylum BASIDIOMYCOTA R.T. Moore

Subphylum AGARICOMYCOTINA Doweld

Class Agaricomycetes Doweld

Agaricales Underw. 
Agaricaceae Chevall.

Coccobotrys Boud. \& Pat. 1900 (H)

Fistulinaceae Lotsy

Confistulina Stalpers 1983 (H)

Lyophyllaceae Jülich

Termitosphaera Cif. 1935 (H)

Mycenaceae Overeem

Decapitatus Redhead \& Seifert 2000 (H)

Niaceae Jülich

Peyronelina P.J. Fisher et al. 1976 (H)

Physalacriaceae Corner

Mycotribulus Nag Raj \& W.B. Kendr. 1970 (C)

Pleurotaceae Kühner

Antromycopsis Pat. \& Trab. 1897 et al. (H)

Nematoctonus Drechsler 1941 et al. (H)

Psathyrellaceae Vilgalys et al.

Hormographiella Guarro \& Gené 1992 (H)

Rhacophyllus Berk. \& Broome 1871 (H)

Tricholomataceae R. Heim ex Pouzar

Moniliophthora H.C. Evans et al. 1978 (H)

Nothoclavulina Singer 1970 (H)

Tilachlidiopsis Keissl. 192 (H)

Tilachlidiopsis Keissl. 1924 (H)

Ugola Adans. 1763 (H)

Agaricales genera incertae sedis

Aleurocystis Lloyd ex G. Cunn. 1956 (C)

Disporotrichum Stalpers 1984

Fibulochlamys A.I. Romero \& Cabral 1989 (H)

Tricladiomyces Nawawi 1985 (H)

Atheliales Jülich

Atheliaceae Jülich

Digitatispora Doguet 1962 (H)*

Fibularhizoctonia G.C. Adams \& Kropp 1996 (H)

Taeniospora Marvanová 1977 (H)

Auriculariales J. Schröt.

Exidiaceae R.T. Moore

Ovipoculum Zhu L. Yang \& R. Kirschner 2010 (H)

Auriculariales genera incertae sedis

Disporotrichum Stalpers 1984 (H)

Oliveorhiza P. Roberts 1998 (H) 
Porpopycnis R. Kirschner 2012 (C)

Cantharellales Gäum

Botryobasidiaceae Jülich

Haplotrichum Link 1824 (H)

Ceratobasidiaceae G.W. Martin

Acanthellorhiza P. Roberts 1999 (H)

Ceratorhiza R.T. Moore 1987 (H)

Rhizoctonia DC. 1805 (H)

Hydnaceae Chevall.

Burgella Diederich \& Lawrey 2007 (H)

Burgoa Goid. 1938 (H)

Ingoldiella D.E. Shaw 1972 (H)

Osteomorpha G. Arnaud ex Watling \& W.B. Kendr. 1979 (H)

Oliveoniaceae P. Roberts 1998

Oliveorhiza P. Roberts 1998 (H)

Tulasnellaceae Juel

Epulorhiza R.T. Moore 1987 (H)

Cantharellales genera incertae sedis

Minimedusa Weresub \& P.M. LeClair 1971 (H)

Corticiales K.H. Larss.

Corticiaceae Herter

Basidiodesertica Maharachch. et al. 2020 (H)

Chrysorhiza T.F. Andersen \& Stalpers 1996 (H)

Erythricium J. Erikss. \& Hjortstam 1970 (H)

Giulia Tassi 1904 (C)

Marchandiomyces Dieder. \& D. Hawksw. 1990 (H)

Michenera Berk. \& M.A. Curtis 1868 (H)

Necator Massee 1898 (H)

Tretopileus B.O. Dodge 1946 (H)

Hymenochaetales Oberw.

Schizoporaceae Jülich

Echinodia Pat. 1918 (H)

Hymenochaetales genus incertae sedis

Caeruleomyces Stalpers 2000 (H)

Polyporales Gäum.

Fomitopsidaceae Jülich

Ptychogaster Corda 1838 (H)

\section{Ganodermataceae Donk}

Thermophymatospora Udagawa et al. 1986 (H)

Hyphodermataceae Jülich 
Aegerita Pers. 1801 (H)

Aegeritina Jülich 1984 (H)

Meruliaceae P. Karst.

Bornetina L. Mangin \& Viala 1903 (H)

\section{Phanerochaetaceae Jülich}

Erythricium J. Erikss. \& Hjortstam 1970 (H)

Sporotrichum Link 1809 (H)

Polyporaceae Fr. ex Corda

Digitellus Paulet 1791 (H)

Mycelithe Gasp. 1841 (C)

Pachyma Fr. 1822 (H)

Russulales Kreisel ex P.M. Kirk et al.

Bondarzewiaceae Kotl. \& Pouzar

Spiniger Stalpers 1974 (H)

\section{Peniophoraceae Lotsy}

Licrostroma P.A. Lemke 1964 (H) ${ }^{\# *}$

\section{Stereaceae Pilát}

Matula Massee 1888 (H)

Sebacinales M. Weiss et al.

Sebacinaceae K. Wells \& Oberw.

Chaetospermum Sacc. 1892 (C)

Craterocolla Bref. 1888

Ditangium P. Karst. 1867 (H)

Flahaultiella Seifert 2009 (H)

Opadorhiza T.F. Andersen \& R.T. Moore 1996 (H)

Piriformospora Sav. Verma et al. 1998 (H)

Thelephorales Corner ex Oberw.

Thelephoraceae Chevall.

Parahaplotrichum W.A. Baker \& Partr. 2001 (H)

Agaricomycetes genera incertae sedis

Akenomyces G. Arnaud ex D. Hornby 1984 (H)

Arthrodochium R.F. Castañeda \& W.B. Kendr. 1990 (H)

Arualis Katz 1980 (H)

Bartheletia G. Arnaud ex Scheuer et al. 2008 (H)

Cenangiomyces Dyko \& B. Sutton 1979 (H)

Cruciger R. Kirschner \& Oberw. 1999 (H)

Dendrosporomyces Nawawi et al. 1977 (H)

Ellula Nag Raj 1980 (C)

Fibulocoela Nag Raj 1978 (C)

Fibulotaeniella Marvanová \& Bärl. 1988 (H)

Geotrichopsis Tzean \& Estey 1991 (H)

Gloeosynnema Seifert \& G. Okada 1988 (H)

Glomerulomyces A.I. Romero \& S.E. López 1989 (H) 
Glutinoagger Sivan. \& Watling 1980 (H)

Myriococcum Fr. 1823 (H)

Nyctalina G. Arnaud 1952 (H)

Pagidospora Drechsler 1960 (H)

Pycnovellomyces R.F. Castañeda 1987 (C)

Riessia Fresen. 1852 (H)

Riessiella Jülich 1985 (H)

Titaeella G. Arnaud ex K. Ando \& Tubaki 1985 (H)

Tricladiomyces Nawawi 1985 (H)

Class Dacrymycetes Doweld

Dacrymycetales Henn.

Dacrymycetaceae J. Schröt.

Cerinosterus R.T. Moore 1987 (H)

Dacryoscyphus R. Kirschner \& Zhu L. Yang 2005 (H)

Class Tremellomycetes Hibbett et al.

Cystofilobasidiales Fell et al.

Cystofilobasidiaceae K. Wells \& Bandoni

Guehomyces Fell \& Scorzetti 2004 (H)

Itersonilia Derx 1948 (H)

Rhodozyma Phaff et al. 1972 (H)

Mrakiaceae X.Z. Liu et al.

Mrakiella Margesin \& Fell 2008 (H)

Phaffia M.W. Mill. et al. 1976 (Y)

Tausonia Babeva 1998 (H)

Udeniomyces Nakase \& Takem. 1992 (Y)

Filobasidiales Jülich

Filobasidiaceae L.S. Olive

Cryptococcus Vuill. 1901 (Y)

Tremellales Fr.

Cryptococcaceae Kütz. ex Castell. \& Chalm.

Teunia Q.M. Wang \& F.Y. Bai 2020 (Y)

Cuniculitremaceae J.P. Samp. et al.

Fellomyces Y. Yamada \& I. Banno 1984 (Y)

Kockovaella Nakase et al. 1991 (Y)

Sterigmatosporidium G. Kraep. \& U. Schulze 1983 (Y)

Tremellina Bandoni 1986 (H)

Hyaloriaceae Lindau

Helicomyxa R. Kirschner \& Chee J. Chen 2004 (H)

\section{Tremellaceae Fr.}

Anastomyces W.P. Wu et al. 1997 (H)

Bullera Derx 1930 (Yeast)

Cryptococcus Vuill. 1901 (Y)

Hormomyces Bonord. 1851 (H)

Biatoropsis Räsänen 1934 with hormomyces-like asexual morph $(\mathbf{H})^{\# *}$ 
Tsuchiyaea Y. Yamada et al. 1988 (H)

Trichosporonaceae Nann.

Aegeritella Bałazy \& J. Wiśn. 1974 (H)

Trichosporon Behrend 1890 (Y)

Tritirachium Limber 1940 (H)

Tremellales genera incertae cedis

Derxomyces F.Y. Bai \& Q.M. Wang 2008 (Y)

Hannaella F.Y. Bai \& Q.M. Wang 2008 (Y)

Moniliella Stolk \& Dakin 1966 (H)

Tremellomycetes genera incertae sedis

Heteromycophaga P. Roberts 1997 (Y)

Moniliella Stolk \& Dakin 1966 (H)

Trichosporonoides Haskins \& J.F.T. Spencer 1967 (H)

AGARICOMYCOTINA genus incertae sedis

Microstella K. Ando \& Tubaki 1984 (H)

Subphylum PUCCINIOMYCOTINA R. Bauer et al.

Class Agaricostilbomycetes R. Bauer et al.

Agaricostilbales Oberw. \& R. Bauer

Agaricostilbaceae Oberw. \& R. Bauer

Agaricostilbum J. Wright 1970 (H)

Bensingtonia Ingold 1986 (Y)

Sterigmatomyces Fell 1966 (Y)

Chionosphaeraceae Oberw. \& Bandoni

Ballistosporomyces Nakase et al. 1989 (Y)

Boekhoutia Q.M. Wang \& F.Y. Bai 2020 (Y)

Kurtzmanomyces Y. Yamada et al. 1989 (Y)

Mycogloea L.S.Olive 1950 (Y)

Jianyuniaceae Q.M. Wang \& F.Y. Bai

Pseudosterigmatospora Q.M. Wang \& F.Y. Bai 2020 (Y)

Sterigmatospora Q.M. Wang \& F.Y. Bai 2020 (Y)

Class Atractiellomycetes R. Bauer et al.

Atractiellales Oberw. \& Bandoni

Phleogenaceae Gäum.

Atractiella Sacc. 1886 (H)

Basidiopycnides J. Reid et al. 2008 (H)

Saccoblastiaceae Jülich

Infundibura Nag Raj \& W.B. Kendr. 1981 (H)

Atractiellales genera incertae sedis

Atractogloea Oberw. \& Bandoni 1982 (H)

Hobsonia Berk. ex Massee 1891 (H)

Leucogloea R. Kirschner 2004 (H)

Tricladiomyces Nawawi 1985 (H) 
Pachnocybales R. Bauer et al.

Pachnocybaceae Oberw. \& R. Bauer Pachnocybe Berk. 1836 (H)

Class Classiculomycetes R. Bauer et al.

Classiculales R. Bauer et al.

Classiculaceae Bauer et al.

Jaculispora H.J. Huds. \& Ingold 1960 (H)

Naiadella Marvanová \& Bandoni 1987 (H)

Class Cryptomycocolacomycetes $\mathrm{R}$. Bauer et al.

Cryptomycocolacales Oberw. \& R. Bauer

Cryptomycocolacaceae Oberw. \& R. Bauer

Colacosiphon R. Kirschner et al. 2001 (H)

Class Cystobasidiomycetes R. Bauer et al.

Erythrobasidiales genera incertae sedis

Bannoa Hamam. 2002 (Y)

Erythrobasidium Hamam. et al. 1988 (Y)

Cystobasidiales R. Bauer et al.

Begerowomyces Q.M. Wang \& F.Y. Bai (Y)

Robertozyma Q.M. Wang \& F.Y. Bai (Y)

Cystobasidiomycetes genus incertae sedis

Cyrenella Goch. 1981 (H)

Class Microbotryomycetes R. Bauer et al.

Heterogastridiales Oberw. \& R. Bauer

Heterogastridiaceae Oberw. \& R. Bauer

Hyalopycnis Höhn. 1918 (C)

Kriegeriales Toome \& Aime

Camptobasidiaceae R.T. Moore

Cryolevonia Pontes et al. 2020 (Y)

Glaciozyma Turchetti et al. 2020 (Y)

Leucosporidiales J.P. Samp. et al.

Leucosporidiaceae Jülich

Leucosporidiella Samp. 2003 (Y)

Microbotryales genus incertae sedis

Reniforma Pore \& Sorenson 1990 (Y)

Rosettozymales Q.M. Wang \& F.Y. Bai

Rosettozymaceae Q.M. Wang \& F.Y. Bai

Rosettozyma Q.M. Wang \& F.Y. Bai 2020 (Y)

Sporidiobolales Doweld

Sporidiobolaceae R.T. Moore

Blastoderma B. Fisch. \& Brebeck 1894 (Y)

Rhodomyces Wettst. 1885 (H) 
Sporobolomyces Kluyver \& C.B. Niel 1924 (H)

Sporidiobolales genera incertae cedis

Erythrobasidium Hamam. et al. (Y)

Rhodotorula F.C. Harrison 1927 (Y)

Microbotryomycetes genus incertae sedis

Crucella Marvanová \& Suberkr. 1990 (H)

Class Pucciniomycetes R. Bauer et al.

Helicobasidiales R. Bauer et al.

Helicobasidiaceae P.M. Kirk

Thanatophytum Nees 1816 (H)

Tuberculina Tode ex Sacc. 1880 (H)

Platygloeales R.T. Moore

Eocronartiaceae Jülich

Glomopsis D.M. Hend. 1961 (H)

Platygloeaceae Racib.

Infundibura Nag Raj \& W.B. Kendr. 1981 (H)

Leucogloea R. Kirschner 2004 (H)

Uncolaceae Buriticá

Calidion Syd. \& P. Syd. 1919 (R)

Pucciniales Clem. \& Shear

Coleosporiaceae Dietel

Chrysomyxa Unger 1840 (R)

Cronartiaceae Dietel

Cronartium Fr. 1815 (R)

Crossopsoraceae Aime \& McTaggart

Neoolivea Aime \& McTaggart 2020 (R)

Phakopsoraceae Cummins \& Hirats. f.

Aeciure Buriticá \& J.F. Hennen 1994 (R)

Macabuna Buriticá \& J.F. Hennen 1994

Malupa Y. Ono et al. 1992 (R)

Milesia F.B. White 1878 (R)

Physopella Arthur 1906 (R)

Uredendo Buriticá \& J.F. Hennen 1994 (R)

Uredostilbe Buriticá \& J.F. Hennen 1994 (R)

Phragmidiaceae Corda

Gerwasia Racib. 1909 (R)

Physonema Lév. 1847 (R)

Puccineaceae Chevall.

Caeoma Link 1809 (R)

Roestelia Rebent. 1804 (R) 
Pucciniastraceae Gäum. ex Leppik

Milesia F.B. White 1878 (R)

Peridiopsora Kamat \& Sathe 1969 (R)

Pomatomyces Oerst. 1864 (R)

Pucciniastrum G.H. Otth 1861 (R)

Uropyxidaceae Cummins \& Y. Hirats.

Canasta A.A. Carvalho \& J.F. Hennen 2010 (R)

Pucciniales genera incertae sedis

Elateraecium Thirum. et al. 1966 (R)

Intrapes J.F. Hennen \& Figueiredo 1979 (R)

Uraecium Arthur 1933 (R)

Uredo Pers. 1801 (R)

Septobasidiales Couch ex Donk

Septobasidiaceae Racib.

Johncouchia S. Hughes \& Cavalc. 1983 (H)

Subphylum USTILAGINOMYCOTINA R. Bauer et al.

Class Exobasidiomycetes Begerow et al.

Doassansiales R. Bauer \& Oberw.

Doassansiaceae R.T. Moore ex P.M. Kirk et al.

Savulescuella Cif. 1959 (H)

Entylomatales R. Bauer \& Oberw.

Entylomataceae R. Bauer \& Oberw.

Entylomella Höhn. 1924 (H)

Tilletiopsis Derx 1948 (H)

Exobasidiales Henn.

Brachybasidiaceae Gäum.

Meira Boekhout et al. 2003 (H)

Cryptobasidiaceae Malençon ex Donk

Acaromyces Boekhout et al. 2003 (H)

Exobasidiales genera incertae sedis

Cladostergigma Pat. 1892 (H)

Clinoconidium Pat. 1898 (H)

Coniodictyum Har. \& Pat. 1909 (H)

Golubeviales Q.M. Wang et al.

Golubeviaceae Q.M. Wang et al. 2015

Golubevia Q.M. Wang et al. 2015 (H)

Microstromatales R. Bauer \& Oberw.

Microstromataceae Jülich

Microstroma Niessl 1861 (H)

Quambalariaceae Z.W. de Beer et al.

Quambalaria J.A. Simpson 2000 (H) 
Microstromatales genus incertae sedis

Sympodiomycopsis Sugiy. et al. 1991 (Y)

Class Spiculogloeomycetes Q.M. Wang et al.

Spiculogloeomycetes genus incertae sedis

Meniscomyces Q.M. Wang \& F.Y. Bai 2020 (Y)

Class Ustilaginomycetes R. Bauer et al.

Urocystidales R. Bauer \& Oberw.

Glomosporiaceae Cif.

Rhombiella Liro 1939 (H)

Thecaphorella H. Scholz \& I. Scholz 1988 (H)

Ustilaginales $\mathrm{G}$. Winter

Anthracoideaceae Denchev

Crotalia Liro 1938 (Y)

Cintractiellaceae Vánky

Naiadella Marvanová \& Bandoni 1987 (H)

Ustilaginaceae Tul. \& C. Tul.

Pseudozyma Bandoni emend. Boekhout 1985 (H)

Ustilaginomycetes order incertae sedis

Urocystidiales R. Bauer \& Oberw.

Urocystidaceae Begerow et al.

Paipalopsis J.G. Kühn 1882 (H)

USTILAGINOMYCOTINA, order incertae sedis

Malasseziales R.T. Moore

Malasseziaceae Denchev \& R.T. Moore

Malassezia Baill. 1889

USTILAGINOMYCOTINA genera incertae sedis Botryoconis Syd. \& P. Syd. 1906 (H)

Tilletiella Zambett. 1970 (H)

BASIDIOMYCOTA class incertae sedis

Class Wallemiomycetes Zalar et al.

Wallemiales Zalar et al.

Wallemiaceae R.T. Moore

Wallemia Johan-Olsen 1887 (H)

Anguillomyces Marvanová \& Bärl. 2000 (H)

Arcispora Marvanová \& Bärl. 1998 (H)

Bartheletica G. Arnaud 2008 (H)

Gloeosynnema Seifert \& G. Okada 1988 (H)

Lactydina Subram. 1978 ('1977') (H)

Microstella K. Ando \& Tubaki 1984 (H)

Nodulospora Marvanová \& Bärl. 2000 (H)

Trichosporonoides Haskins \& J.F.T. Spencer 1967 (H)

Stachycoremium Seifert 1986 (H)

Stauriella Sivichai \& E.B.G. Jones 2004 (H) 
Phylum Calcarisporiellomycota Tedersoo et al.

Sub Phylum Calcarisporiellomycotina Tedersoo et al.

Class Calcarisporiellomycetes Tedersoo et al.

Calcarisporiellales Tedersoo et al.

Calcarisporiellaceae Tedersoo et al.

Calcarisporiella de Hoog 1974 (H)

\section{Notes for pleomorphic genera}

Acanthohelicospora Boonmee \& K.D. Hyde

This genus was introduced by Boonmee et al. (2014) who show it to reside in Tubeufiaceae. $\mathrm{Lu}$ et al. (2017) introduced Acanthohelicospora aurea, a hyphomycetous taxon resided to Acanthohelicospora sensu stricto.

\section{Acarosporina Sherwood 1977}

Johnston (1985) reported A. microspora (R.W. Davidson \& R.C. Lorenz) Sherwood with a phacidiella-like, coelomycetous asexual morph.

\section{Acrocordia A. Massal.}

Hongsanan et al. (2020b) reported conidiomata (rare) from this genus.

\section{Acrogenospora M.B. Ellis}

Ellis $(1971,1976)$ and Schoch et al. (2009) regarded Farlowiella as the sexual morph of Acrogenospora. Wijayawardene et al. (2020) proposed Farlowiella, as the older sexually typified name over Acrogenospora. However, Rossman et al. (2015b) did not accept this synonymy and proposed to adopt Acrogenospora over Farlowiella. Hongsanan et al. (2020b) provided the description for the holomorph of the genus. Bao et al. (2020) described several new species under Acrogenospora. We therefore agree that Acrogenospora should therefore be adopted over Farlowiella.

\section{Acrospermum Tode}

Carmichael et al. (1980) reported Acrospermum compressum Tode to have a Dactylaria Sacc. asexual morph. However, Dactylaria is considered as polyphyletic (Seifert et al. 2011), and Dactylaria sensu stricto is placed in Helotiales (Wijayawardene et al. 2020). Hence, we conclude that Acrospermum has dactylaria-like asexual morphs.

Aithaloderma Syd. \& P. Syd.

Kirk et al. (2008) regarded Ciferrioxyphium Bat. \& H. Maia 1963 as the asexual morph of this genus, thus Chomnunti et al. (2011) proposed to adopt older, sexual generic name i.e., Aithaloderma.

\section{Akanthomyces Lebert 1858}

Hodge (2003) reported that this genus 'produces white, cream or flesh-colored, cylindrical, attenuated synnemata covered with a hymenium of phialides and unicellular, hyaline conidia, in short or long chains'.

\section{Albertiniella Kirschst.}

Kirk et al. (2008) reported an acremonium-like asexual morph from this genus.

\section{Alfaria Crous et al. 2014}

This genus was introduced as a sexually typified genus by Crous et al. (2014a), but subsequent studies by Lombard et al. (2016) and Wijayawardene et al. (2016) reported asexual morphs as hyphomycetous and coelomycetous respectively. 
Alina Racib

Sivanesan (1981), Kirk et al. (2008) and Seifert et al. (2011) reported hyphomycetous asexual morphs (Septoidium sp., Shivomyces sp.) from this genus.

Alternaria Nees

Wijayawardene et al. (2014a) and Rossman et al. (2015b) proposed to adopt the older asexually typified name over younger sexually typified name, i.e., Lewia M.E. Barr \& E.G. Simmons 1986 (Art. F.8).

Amarenographium O.E. Erikss.

Eriksson (1982) treated Amarenomyces ammophilae (Lasch) O.E. Erikss., the type species of Amarenomyces as the sexual morph of Amarenographium metableticum, the type species of Amarenographium. Phookamsak et al. (2014) and Wijayawardene et al. (2014a) proposed to adopt Amarenographium over Amarenomyces (Art. F.8). This was followed by Rossman et al. (2015b). However, Hyde et al. (2017) introduced Amarenomyces dactylidis Mapook et al. and showed that Amarenographium and Amarenomyces are not congeneric. Hence, Hyde et al. (2017) reinstated Amarenomyces. Hongsanan et al. (2020a) and Wijayawardene et al. (2020) followed Hyde et al. (2017).

Amniculicola Y. Zhang ter \& K.D. Hyde

Rossman et al. (2016) proposed to adopt Amniculicola, the younger sexually typified name over Anguillospora Ingold 1942 as it needed few name changes.

Amorphotheca Parbery 1969 (= Hormoconis Arx \& G.A. de Vries 1973)

Parbery (1969) introduced this genus as the sexual morph of Cladosporium resinae (Lindau) G.A. de Vries 1955 (Basionym: Hormodendrum resinae Lindau 1906 [1907]). Arx (1973) introduced Hormoconis Arx \& G.A. de Vries to accommodate Hormodendrum resinae Lindau, as a new combination, Hormoconis resinae (Lindau) Arx \& G.A. de Vries. Hence, Hormoconis resinae is the asexual morph of Amorphotheca resinae Parbery, the type species of Amorphotheca. The older name is adopted over the younger name, i.e., Amorphotheca Parbery 1969 over Hormoconis Arx \& G.A. de Vries 1973.

Amphisphaeria Ces. \& De Not.

Amphisphaeria sorbi Senan. \& K.D. Hyde was introduced with coelomycetous asexual morphs (Liu et al. 2015).

Amplistroma Huhndorf et al.

Huhndorf et al. (2009) introduced this genus with acrodontium-like asexual morph.

Angustimassarina Thambug. et al.

Thambugala et al. (2015b) introduced this genus with a hyphomycetous asexual morph.

Anisomeridium (Müll. Arg.) M. Choisy

Hongsanan et al. (2020b) reported conidiomata from this genus.

Annulohypoxylon Y.M. Ju et al.

Annulohypoxylon nitens (Ces.) Y.M. Ju \& J.D. Rogers was reported with nodulosporium-like asexual morphs from culture (Liu et al. 2015).

Antealophiotrema A. Hashim. \& Kaz. Tanaka

Hashimoto et al. (2017) introduced this holomorphic genus (in Pleosporales) with a hyphomycetous asexual morph. 
Anthodidymella Phukhams. et al.

Phukhamsakda et al. (2020) introduced this genus with a coelomycetous asexual morph.

Anthosulcatispora Phukhams. \& K.D. Hyde

Phukhamsakda et al. (2020) introduced this holomorphic genus with a coelomycetous asexual morph.

\section{Apiognomonia Höhn.}

Rossman et al. (2015a) proposed to adopt the younger, sexually typified name over the older asexually typified name, Discula Sacc. 1884 since Apiognomonia errabunda is the cause of anthracnose of oak and of various hardwood trees.

Apiosphaeria Höhn. 1909

Guterres et al. (2018) reported an asexual morph for Apiosphaeria guaranitica, which is the type species of Apiosphaeria.

\section{Apiosporina Höhn.}

Sivanesan (1984) reported a Cladosporium sp. asexual morph from A. collinsii (Schwein.) Höhn., the type species of the genus. However, this taxon was assigned to Fusicladium sensu lato by Braun et al. (2003). Zhang et al. (2011) followed the conclusion in Braun et al. (2003) and accepted that Apiosporina has a fusicladium-like asexual morph.

Apiosporopsis (Traverso) Mariani.

Senanayake et al. (2017a) reported an asexual morph from A. carpinea (Fr.) Mariani based on Potebnia (1910).

Aquadictyospora Z.L. Luo et al.

Li et al. (2017) introduced this aquatic hyphomycetous genus from China. Phylogenetic analyses confirmed its placement in Dictyosporiaceae.

Aquanectria L. Lombard \& Crous

Lombard et al. (2015) introduced this genus with a hyphomycetous asexual morph.

Aquaphila Goh et al.

Boonmee et al. (2014) reported the sexual morph of this genus.

Aquasubmersa K.D. Hyde \& Huang Zhang

Ariyawansa et al. (2015) introduced A. japonica A. Hashim. \& Kaz. Tanaka as a holomorph.

Arachnocrea Z. Moravec.

This genus was reported with a holomorph and the asexual morph resembles Verticillium (Kirk et al. 2008, Hyde et al. 2020b).

\section{Arthrinium Kunze}

Réblová et al. (2016a) proposed to adopt the older, asexually typified name, Arthrinium, over the younger, sexually typified name Apiospora Sacc. 1875.

\section{Arthrobotrys Corda}

Baral et al. (2017) proposed to adopt the older, asexually typified name over Orbiliella Kirschst. 1938.

Arxotrichum A. Nováková \& M. Kolařik 
Crous et al. (2018) introduced this genus with A. wyomingense A. Nováková \& M. Kolařik as the type species. At the same time, Crous et al. (2018) introduced the second species as a new combination, as the Arxotrichum wyomingense which clustered with Chaetomium succineum, a sexual morph.

\section{Aschersonia Mont.}

Rossman et al. (2016) proposed to adopt the younger sexually typified name, Hypocrella Sacc. 1878, over Aschersonia Mont. 1848, the older, asexually typified name. However, Hyde et al. (2020b) did not agree with this adoption and propoed to adopt older, asexually typified name. We also agree with Hyde et al. (2020b) since Aschersonia is the older name and it has more species epithets in Species Fungorum (2021) (55 vs. 54) and it has nore Google Scholar hits (i.e., widely used) (3430 vs. 1590).

\section{Ascocalyx Naumov}

Johnston et al. (2014) followed Groves (1936) and accepted that A. abietis Naumov, the type species of Ascocalyx and Bothrodiscus pinicola Shear 1907, the type of Bothrodiscus Shear 1907 are congeneric. Since Ascocalyx is commonly used and a minor pathogen, Johnston et al. (2014) proposed to adopt it over Bothrodiscus.

Ascochyta Lib.

Chen et al. (2015) reported the holomorph of this genus and provided a comprehensive description.

Ascocodinaea Samuels et al.

Kirk et al. (2008) and Seifert et al. (2011) reported dictyochaeta-like asexual morphs from this genus.

\section{Ascoconidium Seaver}

Johnston et al. (2014) proposed to adopt older, asexually typified name, Ascoconidium over Sageria A. Funk 1975, the younger, sexually typified name.

Ascocoryne J.W. Groves \& D.E. Wilson

Johnston et al. (2014) proposed to adopt Ascocoryne over Coryne Nees 1816, Pirobasidium Höhn. 1902, Pleurocolla Petr. 1924 and Endostilbum Malençon 1964. However, Pleurocolla was accepted as a distinct genus in Nectriaceae (Wijayawardene et al. 2020).

\section{Ascodichaena Butin}

Johnston et al. (2014) proposed to adopt Ascodichaena over Polymorphum Chevall. 1822, Psilospora Rabenh. 1856 and Dichaenopsis Paoli 1905. However, Index Fungorum (2021) listed Dichaenopsis as a synonym of Stagonospora (Sacc.) Sacc.

Ascolacicola Ranghoo \& K.D. Hyde

This genus was introduced with a trichocladium-like asexual morph Ranghoo \& Hyde (1998).

Ascosphaera L.S. Olive et al.

Kirk et al. (2008) reported chrysosporium-like asexual morphs.

Ascotaiwania Sivan. \& H.S. Chang

Yang et al. (2016) introduced Ascotaiwania fusiformis Jing Yang et al. which represents an asexual morph.

Ascotricha Berk. 
Wendt et al. (2017) proposed to adopt Ascotricha, the older sexually typified name over Dicyma Boulanger 1897, the younger, asexually typified generic name.

Ascotrichella Valldos. \& Guarro

Kirk et al. (2008) reported a humicola-like asexual morph from this genus.

Aspergillus P. Micheli ex Link

Houbraken \& Samson (2011) reported the holomorph of the genus Aspergillus.

Asteridiella McAlpine

Hosagoudar \& Riju (2013) reported this genus has phialides produced from hyphae.

Asterinella Theiss.

Kirk et al. (2008) reported this genus with Asterostomula, asteromella-like asexual morphs. However, Wijayawardene et al. (2020) accepted Asterostomula as a distinct genus in Ascomycota genera incertae sedis.

Asterodothis Theiss.

Hongsanan et al. (2014) reported that Asterostromina solaris Bat. \& A.F. Vital, the type species of Asterostromina Bat. \& A.F. Vital is the asexual morph of this genus.

Astrothelium Eschw. genus.

Hongsanan et al. (2020b) reported a coelomycetous asexual morph from this lichenized

Atkinsonella Diehl

Ginns (2011) reported an acremonium-like asexual morph from this genus.

Atrocalyx A. Hashim. \& Kaz. Tanaka

This genus was introduced with a coelomycetous asexual morph and phylogenetic analyses showed that it belongs in Lophiotremataceae (Hashimoto et al. 2017).

Aureovirgo J.A. van der Linde et al. morph.

This genus was introduced by van der Linde et al. (2016) with a leptographium-like asexual

Austropleospora R.G. Shivas \& L. Morin

This genus was reported with an asexual morph which belongs in Camarosporium Schulzer sensu lato (Morin et al. 2010). Verkley et al. (2014) (originally as Paraconiothyrium but later transferred to this genus by Ariyawansa et al. (2015). Jayasiri et al. (2019) introduced a new species which resembled paraconiothyrium-like asexual morphs.

Auxarthronopsis Rah.

Sharma et al. (2013) introduced this genus with a hyphomycetous asexual morph.

\section{Bactrodesmium Cooke}

Réblová et al. (2020a) showed that this genus belongs in Savoryellales.

\section{Balansia Speg.}

Rossman et al. (2016) proposed to adopt the younger, sexually typified name, Balansia over Ephelis Fr. 1849, the older, asexually typified name. Hyde et al. (2020b) mistakenly included Ephelis Fr. as an accepted genus. Furthermore, Hernández-Restrepo et al. (2016) introduced 
Ephelis tripsaci (D. Mulder \& Arx) Hern.-Restr. \& Crous, apparently it needs a name under Balansia.

Balladyna Racib.

Seifert et al. (2011) reported Clasterosporium sp. and Tretospora sp. as asexual morphs of this genus as conidia were associated with ascomata.

Bambusaria Jaklitsch et al.

Jaklitsch et al. (2015) introduced this genus with a coelomycetous asexual morph.

Bambusicola D.Q. Dai \& K.D. Hyde

Dai et al. (2012) introduced this genus with a coelomycetous asexual morph.

Barbatosphaeria Réblová

Réblová (2007) introduced this genus and reported an asexual morph which resembles Sporothrix and Ramichloridium.

Barrina A.W. Ramaley

This genus was introduced by Ramaley (1997) with a phialophora-like asexual morph.

\section{Barrmaelia Rappaz}

Kirk et al. (2008) reported a libertella-like asexual morph from this genus.

\section{Batistia Cif.}

Réblová et al. (2016a) proposed to adopt the older sexually typified name rather than Acrostroma Seifert 1987, the younger, asexually typified name.

\section{Beauveria Vuill.}

Li et al. (2001), Rehner et al. (2011) and Kepler et al. (2017) reported the sexual morph of this genus as cordyceps-like.

\section{Berkleasmium Zobel}

Hongsanan et al. (2020b) and Wijayawardene et al. (2020) accepted this genus as a member of Tubeufiaceae.

\section{Berkleasmium Zobel}

$\mathrm{Lu}$ et al. (2018) reported that this genus is holomorphic. The sexual morph shows a 'superficial ascomata with dark brown to black multi-celled setae, and cylindrical asci with slightly curved fusiform ascospores' and the asexual morph is hyphomycetous, dictyosporous and helicosporous.

\section{Bettsia Skou}

Kirk et al. (2008) reported chrysosporium-like asexual morphs.

\section{Biatoropsis Räsänen}

Kirk et al. (2008) reported a hormomyces-like asexual morph from this genus.

\section{Biatriospora K.D. Hyde \& Borse}

Hongsanan et al. (2020a) reported the hyphomycetous asexual morph of this genus while introducing B. borsei B. Devadatha \& V.V. Sarma, a hyphomycetous taxon.

Bimuria D. Hawksw. et al. 
Wijesinghe et al. (2020) introduced B. omanensis Wijesinghe et al. which represents a coelomycetous asexual morph.

\section{Bioscypha Syd.}

Kirk et al. (2008) reported a chalara-like asexual morph from this genus.

\section{Bipolaris Shoemaker}

Manamgoda et al. (2012) resolved the complex of Bipolaris, Cochliobolus and Curvularia and showed Bipolaris and Cochliobolus formed a monophyletic clade. Hence, Manamgoda et al. (2012) proposed the use of Bipolaris over Cochliobolus (Art. F.8).

\section{Biscogniauxia Kuntze}

Liu et al. (2015) showed that B. marginata (Fr.: Fr.) Pouzar produces nodulosporium-like asexual morphs in culture.

\section{Blackwellomyces Spatafora \& Luangsa-ard}

Kepler et al. (2017) introduced this genus with a hyphomycetous asexual morph.

Blasdalea Sacc. \& P. Syd.

Petrak (1958) introduced the genus Chrysogloeum to accommodate the asexual morph of Singeriella Petr. Kirk et al. (2008) regarded Singeriella as a synonym of Blasdalea Sacc. Both genera are monotypic. Hence, Wijayawardene et al. (2014a) proposed to adopt the oldest name Blasdalea over Chrysogloeum.

\section{Bloxamia Berk.}

Bloxamia truncata (type species of the genus) was reported from cultures of single ascospore isolations of Bisporella sulphurina (Berthet 1964). Johnston (1998) provided evidence of the connection between a Bloxamia asexual morph through isolation of Bisporella discedens in pure culture. However, the type species of Bisporella has not been sequenced, thus its link with Bloxamia sensu stricto is doubtful.

\section{Blumeria Golovin ex Speer}

Braun (2013) proposed to conserve Blumeria 1974 over Oidium Link 1824, the older asexually typified name.

\section{Blumeriella Arx}

Johnston et al. (2014) proposed to adopt Blumeriella over Microgloeum Petr. 1922 and Phloeosporella Höhn. 1916. According to Johnston et al. (2014), 'The asexual morphs of B. jaapii have been referred to as Phloeosporella padi for the macroconidial morph and Microgloeum pruni for the microconidial morph'. However, both Blumeriella and Phloeosporella lack sequence data, thus the links with Blumeriella are doubtful.

Boeremia Aveskamp et al.

Chen et al. (2015) provided the description of the holomorph of this genus.

\section{Bogoriella Zahlbr.}

Hongsanan et al. (2020b) reported a coelomycetous asexual morph from this lichenized genus.

Botryobambusa Phook. et al.

Liu et al. (2012b) introduced this genus with a coelomycetous asexual morph. 
Botryohypoxylon Samuels \& J.D. Rogers

Rossman et al. (2015a) proposed to adopt this generic name over Iledon Samuels \& J.D. Rogers, the asexually typified name. Both generic names have been introduced in the same publication and represent the sexual and asexual morph of one taxon.

Botryosphaeria Ces. \& De Not.

The genus Botryosphaeria has two different types of coelomycetous asexual morphs viz Fusicoccum and Dichomera (Phillips et al. 2013, Wijayawardene et al. 2016). Thus, Wijayawardene et al. (2014a, 2017b) proposed to reduce both asexual genera under Botryosphaeria.

Botrytis P. Micheli ex Pers.

Johnston et al. (2014) proposed to adopt Botrytis, the older, asexual, typified name over Botryotinia Whetzel 1945, the younger sexually typified name.

Brachysporium Sacc.

Réblová et al. (2016a) proposed to adopt Brachysporium, the asexually typifiedolder, asexually typified name over Cryptadelphia Réblová \& Seifert 2004, younger, sexually typified name.

Bretziella Z.W. de Beer et al.

De Beer et al. (2017) introduced this genus with a hyphomycetous asexual morph.

\section{Brooksia Hansf.}

Moore (1962) introduced Hiospira R.T. Moore to accommodate the conidial morph of Brooksia tropicalis Hansf., the type species of this genus. Hence, Wijayawardene et al. (2014a) proposed to reduce Hiospira, the younger asexually typified name under Brooksia, the sexually typified name.

Brunneiperidium Daranag. et al.

Daranagama et al. (2015) introduced this genus with a hyphomycetous asexual morph.

Brunneocarpos Giraldo \& Crous

Crous et al. (2016a) introduced this genus with a hyphomycetous asexual morph.

Brunneofusispora S.K. Huang \& K.D. Hyde

Wanasinghe et al. (2020) reported a coelomycetous asexual morph for this genus.

Bryochiton Döbbeler \& Poelt

Wäli et al. (2014) reported Bryochiton sp. with chlamydospores-like stages (meristematic morph) from cultures.

Budhanggurabania $\mathrm{P}$. Wong et al.

Crous et al. (2015d) introduced this genus with a coelomycetous asexual morph.

Cainia Arx \& E. Müll.

Senanayake et al. (2015) reported a coelomycetous asexual morph from this genus.

\section{Calloria Fr.}

Johnston et al. (2014) proposed to adopt Calloria over Cylindrocolla Bonord. 1851, the younger asexually typified name. 


\section{Calonectria De Not.}

Lombard et al. (2010) proposed to adopt Calonectria, the older, sexually typified name over Cylindrocladium Morgan 1892, the younger, asexually typified name.

\section{Caloscypha Boud.}

Healy et al. (2016) adopted Caloscypha, the older, sexually typified name over Verticicladium Preuss 1851, the younger asexually typified name.

Calosphaeria Tul. \& c. Tul.

Réblová et al. (2016a) proposed to adopt Calosphaeria, the older, sexually typified name over Calosphaeriophora Réblová et al. 2004, the younger, asexually typified name.

Calostilbe Sacc. \& Syd.

Rossman et al. (1999), Kirk et al. (2008), Seifert et al. (2011) and Lombard et al. (2015) regarded Calostilbella Höhn. as the asexual morph of Calostilbe. Hence, the older, sexually typified name Calostilbe was adopted over the younger, asexually typified name Calostilbella.

\section{Calycellina Höhn.}

Johnston et al. (2014) proposed to adopt Calycellina, sexually typified older name over Chaetochalara B. Sutton \& Piroz. 1965, the younger asexually, typified name.

\section{Camarosporium Schulzer}

Wanasinghe et al. (2017a) provided a comprehensive background of homomorphism of this genus.

\section{Camarosporula Petr.}

Camarosporula Petr. 1954 and Anthracostroma Petr. 1954 are congeneric and published in the same publication. Wijayawardene et al. (2014a) proposed to adopt the sexually typified name, Anthracostroma. However, Rossman et al. (2015b) proposed to adopt Camarosporula as it is reported frequently.

\section{Camporesiomyces D.P. Wei et al.}

Hyde et al. (2020a) introduced this genus with a hyphomycetous morph.

Camptophora Réblová \& Unter.

Yang et al. (2018a) reported sexual morph of Camptophora hylomeconis (Crous et al.), Réblová \& Unter.

\section{Camillea Fr.}

Wendt et al. (2017) proposed to adopt older, sexually typified name Camillea over Xylocladium P. Syd. ex Lindau, the younger, asexually typified name.

Canalisporium Nawawi \& Kuthub.

Réblová et al. (2016a) proposed to adopt older, asexually typified name Canalisporium rather than Ascothailandia Sri-indr. et al. 2010, the younger sexually typified name.

Canomyces Rahul Sharma \& Shouche

Sharma \& Shouche (2020) introduced this genus and reported arthroconidia from the cultures.

\section{Capnodium Mont.}


Chomnunti et al. (2011) confirmed that Polychaeton (Pers.) Lév. 1846 is the asexual morph of this genus thus, regaded as a synonym. Rossman et al. (2015b) proposed to adopt the younger sexually typified name over older asexually typified name.

Caprettia Bat. \& H. Maia

Hongsanan et al. (2020b) reported conidiomata from this genus.

\section{Caproventuria U. Braun}

Zhang et al. (2011) concluded that Pseudocladosporium to be asexual morph of Caproventuria. Hongsanan et al. (2020b) accept Pseudocladosporium as the asexual morph of Caproventuria, but this needs confirmation by phylogenetic analyses.

\section{Ceramothyrium Bat. \& H. Maia}

Constantinescu et al. (1989) introduced Stanhughesia Constant. to accommodate the asexual morph of three species of Ceramothyrium. Stanhughesia carniolica O. Const., S. linnaeae O. Const. and S. lycopodii O. Const. were introduced as the asexual morphs of Ceramothyrium carniolicum (Rehm) Petrak., C. linnaeae (Dearn.) Hughes and C. lycopodii K. \& L. Holm respectively. The links between morphs were confirmed by culture studies (Constantinescu et al. 1989) thus, younger asexually typified name, i.e., Stanhughesia is regarded as a synonym of older sexually typified name i.e., Ceramothyrium.

Ceratocystiopsis H.P. Upadhyay \& W.B. Kendr.

Upadhyay \& Kendrick (1975) introduced this genus and its asexual morph Hyalorhinocladiella H.P. Upadhyay \& W.B. Kendr. Ceratocystiopsis was published prior to Hyalorhinocladiella thus, former, sexually typified name is adopted over the latter, younger asexual name.

Ceratocystis Ellis \& Halst. morphs.

Kirk et al. (2008) and Hyde et al. (2020b) reported chalara-like and thielaviopsis-like asexual

Ceratosphaerella Huhndorf et al.

Hyde et al. (2020b) reported a hyphomycetous asexual morph from this genus.

\section{Ceratosphaeria Niessl}

Crous et al. (2020a) accepted this genus with a coelomycetous asexual morph.

\section{Cercophora Fuckel}

This sexually typified genus has been linked with several asexual genera such as Phialophora, Cladorrhinum (Kirk et al. 2008, Siefert et al. 2011). Ueda (1994) reported a chrysosporium-like asexual morph from Cercophora terricola S. Ueda.

\section{Chaenothecopsis Vain.} 2011).

This genus was reported as the sexual morph of Catenomycopsis Constant. 1991 (Seifert et al.

\section{Chaetomella Fuckel}

Johnston et al. (2014) proposed to adopt asexually typified older name, Chaetomella over sexually typified younger name, Zoellneria Velen. 1934.

Chaetomidium (Zopf) Sacc.

Kirk et al. (2008) reported this genus with a phialophora-like asexual morph. 


\section{Chaetomium Kunze}

Réblová et al. (2016a) proposed to adopt Chaetomium, the older sexually typified generic name over the younger, asexually typified genera Trichocladium Harz 1871, Botryotrichum Sacc. \& Marchal 1885 or Humicola Traaen 1914.

Chaetoplea (Sacc.) Clem.

Phookamsak et al. (2014) observed a coelomycetous morph closely associated with the sexual morph on the lectotype. Hence, the holomorph of Chaetoplea was established based on cooccurrence of both morphs on same host material. Moreover, Phookamsak et al. (2014) treated Chaetoplea as a member of Leptosphaeriaceae based on morphological characters.

Chaetorostrum Zelski et al.

Zelski et al. (2011) introduced this genus with a hyphomycetous asexual morph.

Chaetosphaerella E. Müll. et al.

Réblová et al. (2016a) proposed to adopt Chaetosphaerella over Oedemium Link 1824 and Veramycina Subram. 1995. Both suppressed genera are asexually typified and Oedemium is the older generic name. However, Réblová et al. (2016a) adopted Chaetosphaerella as it was frequently used rather than Oedemium.

Chaetosphaeria Tul. \& C. Tul.

Réblová \& Winka (2000) and Fernández et al. (2006) reported a hyphomycetous asexual morph from this genus.

\section{Chaetosphaerides Matsush.}

Matsushima (2001) introduced this genus and reported a ramichloridium-like asexual morph.

\section{Chiangraiomyces Senan. et al.}

Senanayake et al. (2017a) introduced this genus with a coelomycetous asexual morph.

\section{Chlamydotubeufia Boonmee \& K.D. Hyde}

Boonmee et al. (2011) introduced this genus with a hyphomycetous asexual morph.

\section{Chloridium Link}

Réblová et al. (2016a) proposed to adopt this name over the younger, sexually typified name.

Chlorociboria Seaver ex Ramamurthi et al.

Johnston et al. (2014) proposed to adopt Chlorociboria, the younger, widely used sexually typified name over Dothiorina Höhn. 1911, older asexually typified name.

\section{Chlorovibrissea L.M. Kohn}

This genus was reported with a phialophora-like asexual morph (Kirk et al. 2008).

\section{Chromolaenicola Mapook \& K.D. Hyde} morph.

Mapook et al. (2020) introduced this holomorphic genus with a coelomycetous asexual

\section{Ciliolarina Svrcek}

Kirk et al. (2008) reported this genus with a septonema-like asexual morphs.

\section{Cistella Quél.}

Kirk et al. (2008) reported a phialophora-like asexual morph. 


\section{Cladosporium Link}

Bensch et al. (2012), Wijayawardene et al. (2014a) and Rossman et al. (2015b) proposed to adopt Cladosporium over the younger sexually typified name, Davidiella Crous \& U. Braun 2003.

Clarohilum Videira \& Crous

Videira et al. (2017) introduced this holomorphic genus with a hyphomycetous asexual morph. Morphologically, asexual morph resembles Passalora.

\section{Clasterosporium Schwein}

Zhang et al. (2016) proposed to adopt the older, asexually typified name over younger, sexually typified name, Clasterosphaeria Sivan. 1984.

Clathrospora Rabenh.

Kirk et al. (2008) mentioned that this genus has an alternaria-like asexual morphs but listed as nom. rej. (Kirk et al. 2008).

\section{Claussenomyces Kirschst.}

Johnston et al. (2014) proposed to adopt the younger, sexually typified name Claussenomyces over older, asexually typified name, Dendrostilbella Höhn. 1905

\section{Claviceps Tul.}

Rossman et al. (2013) proposed to adopt the younger, widely used sexually typified name, Claviceps over Sphacelia Lév. 1827 (= Ustilagopsis Speg. 1880), the older, asexually typified name.

Cleistosphaera Syd. \& P. Syd.

Boonmee et al. (2017) reported a coelomycetous asexual morph which was associated with $C$. macrostegia, the type species of this genus.

\section{Clonostachys Corda}

Rossman et al. (2013) proposed to adopt Clonostachys the older, asexually typified name over Bionectria Speg. 1919, the younger, sexually typified name.

Clypeosphaerella Guatim. et al.

Videira et al. (2017) introduced this holomorphic genus with a hyphomycetous (cercosporalike) asexual morph.

\section{Coccinonectria L. Lombard \& Crous}

Lombard et al. (2015) introduced this genus with a hyphomycetous asexual morph.

\section{Coccodinium A. Massal}

Crous et al. (2007a) reported a hyphomycetous asexual morph from this genus.

\section{Coelodictyosporium Thambug. \& K.D. Hyde}

Thambugala et al. (2015b) introduced this holomorphic genus with dictyosporium-like spores. Phylogenetic analyses showed that it belongs in Lophiostomataceae.

\section{Collarispora Videira \& Crous}

Videira et al. (2017) introduced this genus which based on mycosphaerella-like sexual and pseudocercospora-like asexual morph. 
Réblová et al. (2016a) proposed to adopt this genus rather than Glomerella Spauld. \& Schrenk 1903, the younger, sexually typified name.

Collodiscula I. Hino \& Katum.

Wendt et al. (2017) proposed to adopt the older, sexually typified generic name Collodiscula over Acanthodochium Samuels et al. 1987, the younger, asexually typified generic name.

Coma Nag Raj \& W.B. Kendr.

Johnston et al. (2014) proposed to adopt the older, asexually typified generic name Coma, over Ascocoma H.J. Swart 1987, the younger, sexually typified generic name.

Comminutispora A.W. Ramaley

Comminutispora and Hyphospora A.W. Ramaley 1996 are congeneric and introduced in the same publication. Wijayawardene et al. (2014a) and Rossman et al. (2015b) proposed to adopt sexually typified name, Comminutispora over Hyphospora.

\section{Comoclathris Clem.}

Hongsanan et al. (2020a) introduced C. galatellae D. Pem et al. with a coelomycetous asexual morph.

Coniella Höhn.

Alvarez et al. (2016) regarded Pilidiella and Schizoparme as synonyms of the older, asexually typified name, Coniella.

Coniochaeta (Sacc.) Cooke

Réblová et al. (2016a) proposed to adopt this generic name over the younger asexually typified generic name, Lecythophora Nannf. 1934.

Coniolariella D. Garciá et al.

This genus was linked with rhinocladiella-like and dactylaria-like asexual morphs (Kirk et al. 2008, Seifert et al. 2011).

\section{Conioscypha Höhn.}

Réblová et al. (2016a) proposed to adopt Conioscypha, the older, asexually typified name rather than Conioscyphascus Réblová \& Seifert 2004, the younger, sexually typified name.

Conlarium F. Liu \& L. Cai

Liu et al. (2012a) introduced this genus with a hyphomycetous asexual morph.

Cophinforma Doilom et al.

Phillips et al. (2013) stated that Cophinforma sensu stricto comprises two coelomycetous taxa viz. C. mamane and C. atrovirens.

\section{Corallomycetella Henn.}

Herrera et al. (2013) and Lombard et al. (2015) regarded Rhizostilbella Wolk 1914 as the asexual morph of this genus. Corallomycetella is older than Rhizostilbella thus reduced latter under former name (Herrera et al. 2013).

Corallonectria C. Herrera \& P. Chaverri

Herrera et al. (2013) introduced this genus with a hyphomycetous asexual morph.

\section{Cordana Preuss}


Réblová et al. (2016a) proposed to adopt this asexually typified generic name over Porosphaerella E. Müll. \& Samuels 1982, the younger sexually typified name.

Cordyceps (Fr.) Link

Kepler et al. (2017) proposed to adopt this genus name over three asexually typified names viz. Isaria Pers. 1794, Microhilum H.Y. Yip \& A.C. Rath 1989, Evlachovaea Borisov \& Tarasov 1999.

Corinectria C. González \& P. Chaverri

González \& Chaverri (2017) introduced this genus with a hyphomycetous asexual morph.

Cornuvesica C.D. Viljoen et al.

Viljoen et al. (2000) introduced this genus with a chalara-like asexual morph.

Corollospora Werderm.

Réblová et al. (2015) adopted Corollospora, the older, sexually typified name over Varicosporina Meyers \& Kohlm. 1965 and Halosigmoidea Nakagiri et al. 2009, the younger, asexually typified names.

Corylicola Wijesinghe et al.

Wijesinghe et al. (2020) introduced this genus with a coelomycetous asexual morph.

\section{Coryneum Nees}

Rossman et al. (2015a) proposed to adopt Coryneum, the older, asexually typified name over Pseudovalsa Ces. \& De Not. 1863, the younger sexually typified name.

\section{Crassicarpon Y. Marín et al.}

Marin-Felix et al. (2015) introduced this genus with hyphomycetous asexual morph. However, this name in invalid (Art. F.5.1, Shenzhen).

Crassiclypeus A. Hashim. et al.

Hashimoto et al. (2018) introduced this genus with both sexual and asexual morphs. Asexual morph was reported as coelomycetous which showed 'globose to subglobose conidiomata; peridium composed of subglobose to rectangular conidia with rounded ends, hyaline and aseptate' (Hashimoto et al. 2018).

Crassimassarina A. Hashim. \& Kaz. Tanaka

Hashimoto et al. (2017) introduced this holomorphic genus with a coelomycetous asexual morph. Phylogenetic analyses confirmed that it belongs in Lophiotremataceae.

Crassiperidium M. Matsum. \& Kaz. Tanaka

Matsumura et al. (2018) introduced this holomorphic genus with a coelomycetous asexual morph. In phylogenetic analyses, it belongs in Pleosporales genera incertae sedis.

\section{Crassochaeta Réblová}

Réblová (1999) reported an arthrinium-like asexual morph from this genus.

\section{Creosphaeria Theiss.}

Ju et al. (1993) reported Creosphaeria sassafras (Schwein.) Y.M. Ju et al. with asexual morphs which produce conidia from mycelia or in stromata.

\section{Cristulariella Höhn.}


Johnston et al. (2014) proposed to adopt the older, asexually typified name, Cristulariella, over Nervostroma Narumi \& Y. Harada 2006, the younger, sexually typified name.

\section{Crumenulopsis J.W. Groves}

Johnston et al. (2014) proposed to adopt Crumenulopsis, the younger sexually typified, widely used name over Digitosporium Gremmen 1953, the older, asexually typified name. Zhuang (1988) established the link between two genera.

Cryphonectria (Sacc.) Sacc. \& D. Sacc.

Rossman et al. (2015a) proposed to adopt the older, sexually typified name over the younger asexually typified name, Endothiella Sacc. 1906.

Cryptoclypeus A. Hashim. \& Kaz. Tanaka

This genus is a holomorphic genus with a coelomycetous asexual morph which resides in Lophiotremataceae (Hashimoto et al. 2017).

Cryptosporella Sacc. 1877

Rossman et al. (2015a) proposed to adopt the older, sexually typified name over the younger asexually typified name, Disculina Höhn. 1916

Cryptovalsa Ces. \& De Not. ex Fuckel

A coelomycetous asexual morph of Cryptovalsa ampelina (Nitschke) Fuckel was reported by Li et al. (2016).

Cubonia Sacc.

Seifert et al. (2011) reported a papulaspora-like asexual morphs from this genus.

Cucitella Jaklitsch \& Voglmayr

Jaklitsch et al. (2018) introduced this holomorphic genus with a coelomycetous asexual morphs.

\section{Cucurbitaria Gray}

Jaklitsch et al. (2018) reported this genus with a pyrenochaeta-like asexual morph.

\section{Curvularia Boedijn}

Manamgoda et al. (2012) confirmed that Pseudocochliobolus resides in Curvularia sensu stricto. Hence, Manamgoda et al. (2012) proposed the older, asexually typified name, Curvularia over Pseudocochliobolus the younger, sexually typified name.

\section{Cyclopeltella Petr.}

Cyclopeltella was introduced to accommodate the conidial state of Cyclopeltis Petr. in the same publication, but three pages after the sexual state was introduced (Petrak 1953). Cyclopeltis has more epithets than Cyclopeltella (Index Fungorum 2021), hence Wijayawardene et al. (2014a) proposed use of the sexually typified older name, Cyclopeltis over Cyclopeltella, asexually-typified younger name. However, Rossman et al. (2015b) proposed to usage of Cyclopeltella since Cyclopeltis is a later homonym of the fern genus Cyclopeltis J. Sm. 1846 (Aspidiaceae).

Cyclothyriella Jaklitsch \& Voglmayr

Jaklitsch \& Voglmayr (2016) introduced this genus with a coelomycetous asexual morph.

\section{Cylindrocladiella Boesew.}


Lombard et al. (2015) regarded Nectricladiella Crous \& C. L. Schoch, the younger, sexually typified name as a synonym of Cylindrocladiella, the older, asexually typified name.

\section{Cylindrotrichum Bonord.}

Réblová et al. (2016a) proposed to adopt Cylindrotrichum, the older, asexually typified name rather than Reticulascus Réblová \& W. Gams 2011, the younger sexually typified name.

\section{Cyphellophora G.A. de Vries}

Yang et al. (2018a) introduced Cyphellophora jingdongensis H. Yang \& K.D. Hyde which represents a sexual morph.

Dactylidispora Y. Marín et al.

Marin-Felix et al. (2018) introduced this genus with a hyphomycetous asexual morph.

Dactylonectria L. Lombard \& Crous

Lombard et al. (2014b) introduced this genus with a hyphomycetous asexual morph.

Daldinia Ces. \& de Not.

Réblová et al. (2016a) proposed to adopt Daldinia, the older, sexually typified name over Annellosporium M.L. Davey 2010, the younger, asexually typified name.

Davidsoniella Z.W. de Beer et al.

De Beer et al. (2014) introduced this genus with a hyphomycetous asexual morph.

\section{Dematioscypha Svrček}

Johnston et al. (2014) proposed to adopt Dematioscypha, the younger, sexually typified name over Haplographium Berk. \& Broome 1859 (= Schizocephalum 1852), the older, asexually typified name.

\section{Dermea Fr.}

Johnston et al. (2014) proposed to adopt Dermea, the younger, widely used sexually typified name over Sphaeronaema Fr., the older, asexually typified name. However, the type species of Sphaeronaema, S. cylindricum (Tode) Fr. is lacking DNA sequence data thus, its placement is unknown.

\section{Desmazierella Lib.}

Healy et al. (2016) adopted Desmazierella, the older, sexually typified name over Geniculodendron G.A. Salt 1974, the younger, asexually typified name.

\section{Devonomyces Videira \& Crous}

Videira et al. (2017) introduced this holomorphic genus which comprises a hyphomycetous asexual morph.

Diachora Müll. Arg.

Diachora the older, sexually typified name was proposed to adopt rather than Diachorella Höhn. 1918 by Réblová et al. (2016a).

Diaporthe Nitschke

Rossman et al. (2015a) proposed to adopt the older, sexually typified name Diaporthe, over Phomopsis (Sacc.) Bubák 1905, the younger, asexually typified name.

Diaporthosporella C.M. Tian \& Q. Yang 
Yang et al. (2018b) introduced this genus with a coelomycetous asexual morph.

\section{Diatrype Fr.}

Diatrype thailandica R.H. Perera et al. was introduced with a coelomycetous asexual morph by Li et al. (2016)

Dicarpella Syd. \& P. Syd.

Sogonov et al. (2008), Senanayake et al. (2017a) and Hyde et al. (2020b) accepted that this genus has Tubakia asexual morphs. Since the type species of both genera have not been linked, both generic names are used.

Dichlaena Durieu \& Mont.

Houbraken et al. (2020) tentatively accepted this genus in Aspergillaceae. Moreover, Houbraken et al. (2020) followed Ram (1971) who reported that the asexual morph of this genus is not aspergillus-like but a hyphomycete.

\section{Dichoporis Clem.}

Hongsanan et al. (2020b) reported a coelomycetous asexual morph from this genus.

\section{Dictyomeridium Aptroot et al.}

Hongsanan et al. (2020b) mentioned that this genus shows coelomycetous asexual morphs from several species.

Dictyospora Brahamanage et al.

This genus was introduced by Brahmanage et al. (2017) with a hyphomycetous asexual morph and showed that it belongs in Tubeufiaceae.

Didymella Sacc. ex Sacc.

This genus was reported as a holomorphic genus by Chen et al. (2015) and Peyronellaea Goid. ex Togliani 1952 was reported as its synonym.

Didymocyrtis Vain. 1921

Ertz et al. (2015) regarded Diederichia D. Hawksw 2003 and Diederichomyces Crous \& Trakunyingcharoen 2014 as synonyms of Didymocyrtis and accepted Didymocyrtis with phomalike asexual morphs.

\section{Didymosphaeria Fuckel}

Ariyawansa et al. (2014) transferred Paraconiothyrium variabile Riccioni et al. to this genus as Didymosphaeria variabile (Riccioni et al.) Ariyawansa \& K.D. Hyde. Hence, this genus has paraconiothyrium-like asexual morphs.

Didysimulans Tibpromma et al.

Tibpromma et al. (2017) introduced this genus with chlamydospores which are occasionally produced in the culture.

Dimerium (Sacc. \& P. Syd.) McAlpine

Boonmee et al. (2017) reported a hyphomycetous asexual morph on the holotype of $D$. olivaceum, the type species of this genus.

\section{Diplocarpon F.A. Wolf}

Johnston et al. (2014) provided the historical background of Bostrichonema, Diplocarpon, Entomosporium, and Marssonina species. Based on the observations between sexual-asexual 
morphs in old literature, Johnston et al. (2014) proposed to adopt Diplocarpon over other asexually typified genera. However, Bostrichonema and Entomosporium are lacking DNA sequences thus the link established with Diplocarpon is doubtful.

\section{Diplodia Fr.}

Phillips et al. (2013) reported that this genus shows both asexual and sexual morphs.

\section{Discosia Lib.}

Tanaka et al. (2011) introduced Adisciso Kaz. Tanaka et al. as the sexual morph of Discosia sensu stricto Wijayawardene et al. (2016) proposed to reduce Adisciso under Discosia.

\section{Discosphaerina Höhn}

Holm et al. (1999) stated that this genus has asexual morphs belongs in 'Phyllosticta, with holoblastic conidiogenous cells, and phialidic spermatia in Leptodothiorella (Aa 1973); several species form Kabatia (Reusser 1964, Sivanesan 1984)'.

\section{Dlhawksworthia Wanas. et al.}

This genus is holomorphic as Wanasinghe et al. (2018) introduced D. clematidicola (Wanas. et al.) Wanas. et al. (as Hawksworthiana clematidicola Wanas. et al.) with a camarosporium-like asexual morph.

Dolabra C. Booth et al.

Dolabra nepheliae Booth \& Ting was reported with a rhabdospora-like asexual morph (Zalasky et al. 1971).

\section{Dothidella Speg. 1880}

Kirk et al. (2008) reported that Stictochorella Höhn. as the asexual morph of this genus. However, Stictochorella is a synonym of Asteromella Pass. \& Thüm. (Index Fungorum 2021). Currently, only Asteromella pistaciarum has sequence data in GenBank and belongs in Dothideomycetes genera incertae sedis. An ITS sequence of unidentified Dothidella species is also available in GenBank. However, the link between Dothidella sensu stricto and Asteromella sensu stricto needs to be confirmed with newly generated sequences from the type species of each genus.

\section{Dothidotthia Höhn.}

Senwanna et al. (2019) introduced Dothidotthia negundinicola (Crous \& A. Akulov) Senwanna et al., a hyphomycetous taxon which is phylogenetically linked to Dothidotthia sensu stricto.

\section{Dothiopeltis E. Müll.}

Kirk et al. (2008) reported idriella-like, hyphomycetous asexual morph from this genus.

\section{Dothiora Fr.}

Crous \& Groenewald (2017) regarded Dothichiza Lib. ex Roum., Cylindroseptoria Quaedvl. et al. and a hormonema-like synasexual morph as the asexual morphs of Dothiora.

\section{Dothiorella Sacc.}

This asexually typified generic name represents both sexual and asexual morphs (Phillips et al. 2013).

\section{Dysrhynchis Clem.}

Boonmee et al. (2017) reported hyphomycetous asexual morph on the holotype of $D$. pulchella, the type species of the genus. 
Ectodidymella L.W. Hou et al.

Hou et al. (2020) introduced this genus with a coelomycetous asexual morph.

Elsino $\ddot{e}$ Racib.

Wijayawardene et al. (2014) and Rossman et al. (2015b) proposed to adopt the younger, widely used sexually typified name, Elsinoë over Sphaceloma de Bary, the older, asexually typified name.

Emarcea Duong et al.

Crous et al. (2015d) introduced E. eucalyptigena Crous \& M.J. Wingf. as the first asexual species from this genus.

\section{Emericellopsis J.F.H. Beym}

This genus was introduced by Beym (1940) and reported 'conidiophores arising from substratum or aerial hyphae, and hyaline, 1-2-guttulate, elongate-ellipsoidal conidia'. Kirk et al. (2008) stated that asexual morph is acremonium-like.

Endomeliola S. Hughes \& Piroz.

Hughes \& Pirozynski (1994) reported philides and conidia from the mycelium of $E$. dingleyae S. Hughes \& Piroz., the type species of this genus.

Entalbostroma J.D. Rogers \& P.R. Johnst.

Johnston et al. (2016) introduced this genus with a hyphomycetous asexual morph.

Eosphaeria Höhn.

Kirk et al. (2008) reported a phialophora-like asexual morph from this genus.

\section{Eremomyces Malloch \& Caing}

Hongsanan et al. (2020b) reported that E. bilateralis Malloch \& Cain, the type species of this genus with a coelomycetous asexual morphs.

Eriocamporesia R.H. Perera et al.

Hyde et al. (2020a) introduced this genus with a hyphomycetous morph.

Erysiphe R. Hedw. ex DC.

Kirk et al. (2008) and Seifert et al. (2011) listed Pseudoidium Y.S. Paul \& J.N. Kapoor (1986) as a synonym of Oidium. However, Pseudoidium is treated as the asexual morph of Erysiphe (pers. comn. Susumu Takamatsu).

\section{Erythrocarpon Zukal}

Hyde et al. (2020b) reported a hyphomycetous asexual morph from this genus.

\section{Etayoa Diederich \& Ertz}

Ertz et al. (2014) introduced this genus with a hyphomycetous asexual morph.

\section{Eucasphaeria Crous}

Crous et al. (2007b) introduced this genus with an ascochytopsis-like asexual morph.

Eupelte Syd.

Kirk et al. (2008) reported that this genus has Clasterosporium and a septoidium-like asexual 
morph. However, Clasterosporium was confirmed as the asexual morph of Clasterosphaeria thus, former generic name has been adopted over latter (Zhang et al. 2016). Thus, we accept Eupelte with septoidium-like and clasterosporium-like asexual morphs.

\section{Eutiarosporella Crous}

Crous et al. (2015b) introduced this asexually typified genus and showed it belongs to Botryosphaeriaceae. Li et al. (2016) introduced E. dactylidis (Thambug. et al.) Dissan. et al. which belongs in Eutiarosporella sensu stricto represents sexual morph.

\section{Excipulariopsis P.M. Kirk \& Spooner}

Wijayawardene et al. (2014) and Rossman et al. (2015a) proposed to adopt Excipulariopsis, the older, asexually typified name rather than Kentingia Sivan. \& W.H. Hsieh 1989.

\section{Exosporiella P. Karst.}

Sivanesan (1983) introduced Anomalemma Sivan. and at the same time confirmed Exosporiella as its asexual morph. Anomalemma is the younger generic name thus, Wijayawardene et al. (2014a) proposed to conserve Exosporiella over Anomalemma.

\section{Exserohilum K.J. Leonard \& Suggs}

Leonard \& Suggs (1974) introduced this genus and Setosphaeria K.J. Leonard \& Suggs as it's asexual morph. Wijayawardene et al. (2014a) proposed to adopt widely used sexually typified name i.e., Exserohilum. Rossman et al. (2015b) agreed with this adoption.

Fantasmomyces DongHyeon Lee et al.

Crous et al. (2016b) introduced this genus with hyphomycetous asexual morph

Faurelina Locq.-Lin.

Kirk et al. (2008) reported an arthrographis-like asexual morph.

Flabellascoma A. Hashim. et al.

This holomorphic genus with coelomycetous asexual morph was introduced by Hashimoto et al. (2018).

\section{Flabellascus Réblová}

Réblová et al. (2015) introduced this genus with a hyphomycetous asexual morph.

Flagellostrigula Lücking et al.

Hongsanan et al. (2020b) introduced this lichenized, coelomycetous genus.

\section{Flammocladiella Crous et al.}

Crous et al. (2015c) introduced this genus only with a hyphomycetous asexual morph. Lechat \& Fournier (2018) transferred Nectria decora to this genus as F. decora (Wallr.) Lechat \& J. Fourn. and regarded the type species of the genus, Flammocladiella aceris Crous et al. as a synonym of the former species.

Flavobathelium Lücking et al.

Hongsanan et al. (2020b) reported a coelomycetous asexual morph for this genus.

\section{Fragosphaeria Shear}

Shear (1923) introduced this genus with an acremonium-like asexual morph. 


\section{Fusarium Link}

Rossman et al. (2013) proposed to adopt Fusarium, the older asexually typified name rather than Gibberella Sacc. 1877, the younger, sexually typified name.

\section{Fuscostagonospora Kaz.}

Tanaka \& K. Hiray. Hyde et al. (2019) introduced Fuscostagonospora cytisi Jayasiri et al. which represents sexual morph of the genus Fuscostagonospora

\section{Fusicolla Bonord.}

Lombard et al. (2015) reported descriptions for the holomorph of the genus.

\section{Gaeumannomyces Arx et al.}

Zhang et al. (2016) proposed to adopt Gaeumannomyces, the older, sexually typified name over Harpophora Gams 2000, the younger, asexually typified name.

\section{Galeaticarpa A. Hashim. \& Kaz. Tanaka}

Hashimoto et al. (2017) introduced this holomorphic genus with a coelomycetous asexual morph. Phylogenetic analyses showed that it belongs in Lophiotremataceae.

\section{Gelatinipulvinella Hosoya \& Y. Otani}

Johnston et al. (2014) proposed to adopt Stamnaria, the older, sexually typified name over Titaeospora Bubák 1916, the younger, asexually typified name.

\section{Gelatinipulvinella Hosoya \& Y. Otani}

The type species of Gelatinipulvinella, G. astraeicola Hosoya \& Y. Otani is the sexual morph of Aureohyphozyma astraeicola Hosoya \& Y. Otani, the type species of Aureohyphozyma Hosoya \& Y. Otani 1995 (Hosoya \& Otani 1995). Johnston et al. (2014) proposed to adopt Gelatinipulvinella, over Aureohyphozyma.

Gelatinomyces N. Sanoamuang et al.

Sanoamuang et al. (2013) introduced this genus with a phialophora-like hyphomycetous asexual morph. Ekanayaka et al. (2019) and Wijayawardene et al. (2020; in alternative classification of Leotiomycetes) accepted this genus in Leotiales genera incertae sedis.

\section{Gibellula Cavara}

Kepler et al. (2017) stated that this genus has a torrubiella-like sexual morphs.

Gliocephalotrichum J.J. Ellis \& Hesselt.

Lombard et al. (2014a) regarded Leuconectria Rossman 1993 as a synonym of this genus and provided the description for the holomorph.

Gloeotinia M. Wilson et al.

Johnston et al. (2014) proposed to adopt this generic name over Endoconidium Prill. \& Delacr. 1892, the older asexually typified name, mentioning 'the relatively well-known name $G$. granigena would have to be changed'.

Gloniopsis De Not.

Boehm et al. (2009) reported an aposphaeria-like asexual morph from this genus.

\section{Godronia Moug. \& Lév.}

Johnston et al. (2014) proposed to adopt this sexually typified generic name over Sphaeronaema Fr. 1815, Topospora Fr. 1836, Mastomyces Mont. 1848, Clinterium Fr. 1849, 
Fuckelia Bonord. 1864 and Chondropodiella Höhn. 1917.

Godroniopsis Diehl \& E.K. Cash

Johnston et al. (2014) proposed to adopt Godroniopsis, the older, sexually typified name over Cryocaligula Minter 1986, the younger, asexually typified name.

Graphilbum H.P. Upadhyay \& W.B. Kendr.

Upadhyay \& Kendrick (1975) introduced this genus with a hyphomycetous asexual morph.

\section{Graphostroma Piroz.}

This genus was reported with a nodulisporium-like asexual morph (Kirk et al. 2008).

\section{Gremmeniella M. Morelet}

Johnston et al. (2014) proposed to adopt the younger, sexually typified, widely used generic name, Gremmeniella over Brunchorstia Erikss. 1891.

\section{Grovesinia M.N. Cline et al.}

Johnston et al. (2014) proposed to adopt the older, sexually typified name over the younger, asexually typified name.

\section{Gymnascella Peck}

Seifert et al. (2011) stated that Malbranchea Sacc. has Gymnascella sexual morph.

\section{Halocryptosphaeria Dayar. et al.}

Hyde et al. (2020b) introduced this genus with a phomopsis-like asexual morph.

\section{Halodiatrype Dayar. \& K.D. Hyde}

Dayarathne et al. (2020) reported a libertella-like asexual morph from the cultures.

\section{Halojulella Suetrong et al.}

The type species of Halojulella, H. avicenniae (Borse) Suetrong et al. was reported with coelomycetous asexual morph (Ariyawansa et al. 2013).

Halorosellinia Whalley et al.

Whalley et al. (2000) introduced this genus with a geniculosporium-like asexual morph.

\section{Hamigera Stolk \& Samson}

Peterson et al. (2010), Houbraken \& Samson (2011) reported the holomorph of this genus.

\section{Hapalocystis Auersw. ex Fuckel}

Senanayake et al. (2017b) reported the genus with a coelomycetous asexual morph.

\section{Harknessia Cooke}

Crous et al. (2012b) and Senanayake et al. (2017a) accepted Harknessia with wuestneia-like sexual morphs.

\section{Harposporium Lohde}

Quandt et al. (2014) proposed to adopt Harposporium, the older, asexually typified name over Podocrella Seaver 1928, the younger, sexually typified name.

\section{Heleococcum P.M. Jørg.}

Seifert et al. (2011) stated that this genus has acremonium-like and trichothecium-like asexual 
morphs.

Helicangiospora Boonmee et al.

Boonmee et al. (2014) introduced this genus with a helicoma-like asexual morph.

\section{Helicoma Corda}

Boonmee et al. (2014) reported that this genus is pleomorphic.

\section{Helicomyces Link}

Boonmee et al. (2014) provided the description for holomorph of this genus and confirmed its placement in Tubeufiaceae.

\section{Helicosporium Nees}

Boonmee et al. (2014) reported the sexual morph of this genus and confirmed its placement in Tubeufiaceae.

Helicotubeufia Y.Z. Lu \& J.K. Liu

Liu et al. (2018) introduced this genus with a hyphomycetous asexual morph and showed that it belongs in Tubeufiaceae

\section{Helminthosporium Link}

Hongsanan et al. (2020a) and Wijayawardene et al. (2020) regarded that Helminthosporiella Hern.-Restr. et al. 2016 as a synonym of this genus. Voglmayr \& Jaklitsch (2017) accepted Helminthosporium has massarina- or splanchnonema-like sexual morphs.

\section{Hercospora Fr.}

Norphanphoun et al. (2016) provided the description for holomorph of the genus.

Heteroepichlö̈ E. Tanaka et al.

Tanaka et al. (2002) introduced this genus with an ephelis-like asexual morph.

\section{Heterosphaeria Grev.}

Johnston et al. (2014) proposed to adopt this sexually typified generic name which is older than Heteropatella Fuckel 1874, asexually typified name.

\section{Holwaya Sacc.}

Johnston et al. (2014) proposed to adopt the younger, sexually typified name Holwaya over the older, asexually typified name, Crinula Fr. 1821.

\section{Homortomyces Crous \& M.J. Wingf.}

Wijayawardene et al. (2014b) introduced Homortomyces tamaricis from Tamarix gallica based on its asexual morph. Subsequent study by Thambugala et al. (2017a) reported the sexual morph of this species from the same host. DNA sequence analyses confirmed that both morphs represent one taxon.

Huntiella Z.W. de Beer et al.

De Beer et al. (2014) introduced this genus with a hyphomycetous asexual morph.

\section{Hyalopeziza Fuckel}

Kirk et al. (2008) reported a phialophora-like asexual morph.

Hyaliappendispora Senan. et al. 
Senanayake et al. (2017a) introduced this genus with a coelomycetous asexual morph.

\section{Hyalorbilia Baral \& G. Marson}

Baral et al. (2017) proposed to adopt the younger, sexually typified name over the older, asexually typified name, Dwayaangam Subram.

\section{Hyaloscypha Boud.}

Fehrer et al. (2019) reported a hyphomyetous asexual morph from this genus. Moreover, Pseudaegerita J.L. Crane \& Schokn. and Meliniomyces Hambl. \& Sigler 2005 were regarded as synonyms of this genus.

\section{Hyalotiopsis Punith.}

Réblová et al. (2016a) proposed to adopt the older, asexually typified name Hyalotiopsis rather than Ellurema Nag Raj \& W.B. Kendr. 1985, the younger, sexually typified generic name.

Hydeomyces Maharachch. et al.

Maharachchikumbura et al. (2019) introduced this holomorphic genus with a coelomycetous asexual morph.

\section{Hymenoscyphus Gray}

This is genus was linked with several asexual genera in Kirk et al. (2008), viz. Idriella, Geniculospora, Dimorphospora, Helicodendron, Tricladium, Articulospora and scytalidium-like. However, Johnston et al. (2014) did not consider these links since the type species of above asexual genera are not congeneric with type species of Hymenoscyphus. However, non-type species of Hymenoscyphus are reported with different asexual species e.g. Tricladium splendens, type of Tricladium, has a sexual morph named Hymenoscyphus splendens (Johnston et al. 2014).

Hyperdermium J. White et al.

Sullivan et al. (2000) reported microsclerotium-like structures from the cultures of Hyperdermium bertonii.

\section{Hyphodiscus Kirschst.}

Johnston et al. (2014) proposed to adopt Hyphodiscus, the older, sexually typified name over Catenulifera Hosoya 2002, the younger, asexually typified name.

Hypohelion P.R. Johnst.

Johnston et al. (2014) proposed to adopt Godroniopsis, the younger, sexually typified name over Sphaeronaema Fr. 1815, the older, asexually typified name.

\section{Hypomyces (Fr.) Tul. et al.}

Rossman et al. (2013) proposed to adopt the younger, widely used, sexually typified name Hypomyces over the older, asexually typified name, Cladobotryum.

\section{Hypoxylon Bull.}

Réblová et al. (2016a) and Wendt et al. (2017) proposed to adopt the older, sexually typified name Hypoxylon over Nodulisporium Preuss 1849, the younger, asexually typified name.

\section{Hypsostroma Huhndorf}

Hypsostroma saxicola Huhndorf, the type species of Hypsostroma was reported with a pleurophomopsis-like coelomycetous morph (Huhndorf 1992).

Hysterobrevium E. Boehm \& C.L. Schoch 
Jayasiri et al. (2018) introduced Hysterobrevium rosae with a coelomycetous asexual morph.

Ijuhya Starbäck

Kirk et al. (2008) reported an acremonium-like asexual morph from this genus.

Ilyonectria P. Chaverri \& C. Salgado

This genus was reported with a cylindrocarpon-like asexual morph (Chaverri et al. 2011).

Immotthia M.E. Barr conidia.

Hyde et al. (2017) illustrated holomorph of the genus with brown, ellipsoidal, aseptate

Irenopsis F. Stevens

This genus has asexual morph which is directly produced from hyphae (Hongsanan et al. 2015a).

\section{Jattaea Berl}

Damm et al. (2008) introduced Jattaea prunicola and J. mookgoponga which are reported as holomorph and asexual morph respectively.

Juglanconis Voglmayr \& Jaklitsch

Voglmayr et al. (2017) introduced this genus with a coelomycetous asexual morph.

Jumillera J.D. Rogers et al.

Kirk et al. (2008) reported this genus with geniculosporium-like and libertella-like asexual morph.

Juncigena Kohlm. et al.

Réblová et al. (2016a) proposed to adopt Juncigena, the older, sexually typified name rather than Moheitospora Abdel-Wahab et al. 2010, the younger, asexually typified name.

\section{Kalmusia Niessl}

Hongsanan et al. (2020a) reported coniothyrium-like, microsphaeropsis-like and paraconiothyrium-like asexual morphs from this genus.

Kamalomyces R.K. Verma et al.

Phookamsak et al. (2018) introduced K. bambusicola Y.Z. Lu \& K.D. Hyde with a hyphomycetous asexual morph.

Kastanostachys L. Lombard \& Crous

Lombard et al. (2016) introduced this genus with hyphomycetous asexual morph which has stachybotrys-like conidiophores.

Kellermania Ellis \& Everh.

Minnis et al. (2012), Hyde et al. (2013), Wijayawardene et al. (2014a) accepted the older, asexually typified name i.e., Kellermania over the younger, sexually typified name i.e., Planistromella.

Kirschsteiniothelia D. Hawksw.

The type species of this genus, K. aethiops (Sacc.) D. Hawksw. has a hyphomycetous asexual morph (Su et al. 2016). 
Kohlmeyeriopsis Klaubauf et al.

This genus was introduced with a trichocladium-like (hyphomycetous) asexual morph by Klaubauf et al. (2014).

Koorchaloma Subram.

Hyde et al. (2020b) propoed to adopt the older, asexually typified name over the younger, sexually typified name Kananascus Nag Raj 1984.

\section{Kretzschmaria Fr.}

A geniculosporium-like asexual morph was reported with this genus (Kirk et al. 2008).

\section{Kretzschmariella Viégas}

Kirk et al. (2008) reported a mirandina-like asexual morph from this genus.

\section{Labridella Brenckle}

Rossman et al. (2016) proposed to adopt the younger, but frequently used sexually typified name, Labridella over the older, asexually typified name Griphosphaerioma Höhn. 1918

\section{Lambro Racib.}

Senanayake et al. (2017b) reported this genus with a cylindrosporella-like asexual morph.

\section{Lasiodiplodia Ellis \& Everh.}

Phillips et al. (2013) reported the sexual morph of this genus.

Lasionectriella Lechat \& J. Fourn.

Lechat \& Fournier (2016) introduced this genus with an acremonium-like asexual morph.

\section{Lasiosphaeria Ces. \& De Not.}

This genus was linked with Selenosporella G. Arnaud ex MacGarvie, Phialophora Medlar (Seifert et al. 2011).

\section{Lasiosphaeris Clem.}

Réblová et al. (2016a) proposed to adopt the older, sexually typified name rather than the younger, asexually typified name Lasiadelphia Réblová \& W. Gams 2011.

Lasiostemma Theiss. et al.

Kirk et al. (2008) reported Chaetosticta Petr. \& Syd. as the asexual morph of this genus. However, this link was not proved by culture or DNA sequence analyses.

Lentithecium K.D. Hyde et al.

Hyde et al. (2016) introduced Lentithecium unicellulare Abdel-Aziz, a coelomycetous taxon was introduced by Hyde et al. (2016). This was the first report of an asexual taxa which is grouped in Lentithecium sensu stricto.

\section{Leptopeltis Höhn.}

Kirk et al. (2008) reported a leptothyrium-like, coelomycetous asexual morph from this genus.

\section{Leptorhaphis Körb.}

Hyde et al. (2013) reported 'short microconidia and rod-shaped, macroconidia known from some Leptorhaphis species'. 
Leptosphaeria Ces. \& De Not.

Leptosphaeria cirsii Jayasiri et al. was introduced by Hyde et al. (2017) with a coelomycetous asexual morph. Apparently, this was the first time of producing asexual morph in the culture of Leptosphaeria.

\section{Leptosphaerulina McAlpine}

Phookamsak et al. (2013) reported that Leptosphaerulina saccharicola Phookam. et al. has a pithomyces-like asexual state based on cultural studies.

\section{Leptotrochila P. Karst.}

Leptotrochila medicaginis (Fckl) Schiiepp, an important plant pathogenic species has been linked with Sporonema phacidioides, the type species of Sporonema (Sutton 1980). Hence, Johnston et al. (2014) proposed to adopt Leptotrochila over Sporonema. However, sequence data of both these species are unavailable thus this adoption (Johnston et al. 2014) needs further studies.

\section{Leucosphaerina Arx}

This genus was reported with acremonium-like asexual morphs (von Arx 1987, Malloch 1989).

\section{Leveillula G. Arnaud}

Kirk et al. (2008) and Seifert et al. (2011) reported that Oidiopsis Scalia as the asexual morph of this genus.

\section{Licrostroma P.A. Lemke}

Giraldo et al. (2017) proposed to adopt Licrostroma, the younger, sexually typified name over Michenera Berk. \& M.A. Curtis, the older, asexually typified name.

\section{Lignosphaeria Boonmee et al.} morph.

This genus was introduced by Thambugala et al. (2015b) with a coelomycetous asexual

Linocarpon Syd. \& P. Syd.

Kirk et al. (2008) reported a phialophora-like asexual morph from this genus.

\section{Loculistroma F. Patt et al.}

Hyde et al. (2020b) reported a cladosporium-like asexual morph from this genus, following White et al. (2003).

\section{Longididymella L.W. Hou et al.}

This genus was introduced as a holomorphic genus with a coelomycetous asexual morh (Hou et al. 2020).

ongipedicellata $\mathrm{H}$. Zhang et al.

Phukhamsakda et al. (2016) reported a hyphomycetous asexual morph from L. aptrootii (Hyde \& Wong) Zhang et al., the type species of Longipedicellata.

\section{Lopadostoma (Nitschke) Traverso}

Kirk et al. (2008) reported a libertella-like asexual morph from this genus.

\section{Lophiostoma Ces. \& De Not.}

Lophiostoma caulium (Fr.) Ces. \& De Not. was reported with a coelomycetous asexual morph in culture (Ariyawansa et al. 2015). However, the spores were not observed by Ariyawansa 
et al. (2015).

\section{Lophiotrema Sacc.}

Hashimoto et al. (2017) mentioned that this genus has a pycnidial asexual morph.

Lunatiannulus Daranag. et al.

Daranagama et al. (2015) introduced this genus with a libertella-like asexual morph.

\section{Macrodiplodiopsis Petr.}

Crous et al. (2015a) introduced the sexual morph of $M$. desmazieri (Mont.) Petr., the type species of Macrodiplodiopsis.

\section{Magnaporthiopsis J. Luo \& N. Zhang}

Luo \& Zhuang (2012) introduced this genus with a phialophora-like asexual genus.

\section{Magnibotryascoma Thambug. \& K.D. Hyde}

Hyde et al. (2017) introduced M. mali Phukhams. et al., a coelomycetous taxon which grouped in Magnibotryascoma sensu stricto.

Malaysiasca Crous \& M.J. Wingf.

Crous et al. (2016a) introduced this genus with a hyphomycetous asexual morph.

\section{Massarina Sacc.}

Tibpromma et al. (2018a) reported asexual taxon, Massarina pandanicola, which is phylogenetically linked to Massarina sense stricto.

\section{Massariovalsa Sacc.}

Rossman et al. (2015a) proposed to adopt Massariovalsa, the older, sexually typified name over Melanconiopsis Ellis \& Everh. 1900, the younger, asexually typified name.

\section{Mazzantia Mont.}

Réblová et al. (2016a) proposed to adopt the older, sexually typified name over Mazzantiella Höhn. 1918, the younger, asexually typified name.

\section{Mebarria J. Reid \& C. Booth}

Index Fungorum (2021) listed Harknessia thujina Ellis \& Everh. 1894 as the asexual morph of Mebarria thujina (Nag Raj \& DiCosmo) J. Reid \& C. Booth (Basionym: Cryptosporella thujina Nag Raj \& DiCosmo), the type species of this genus.

\section{Megalotremis Aptroot}

Hongsanan et al. (2020b) reported conidiomata from this genus.

\section{Melanconiella Sacc.}

Voglmayr et al. (2012) reported this genus with two types of asexual morphs i.e., discosporina-like and melanconium-like.

Melanconis Tul. \& C. Tul.

Rossman et al. (2015a) proposed to protect the younger, sexually typified names, Melanconis over the older, asexually typified name Melanconium Link 1809 since the generic concept of the latter is not well-established. 
Coelomycetous asexual morphs have been reported from this genus in Hyde et al. (2013) and Tian et al. (2015).

\section{Melanops Nitschke}

Phillips \& Alves (2009) accepted this genus with a fusicoccum-like asexual morph.

\section{Melanopsamma Niess1}

Lombard et al. (2016) reported the holomorph of this genus.

\section{Melanospora Corda}

Marin-Felix et al. (2018) reported an asexual morph with phialidic conidiogenesis and hyaline conidia.

\section{Melanotrigonum Réblová}

Réblová et al. (2016b) introduced this genus with a hyphomycetous asexual morph.

\section{Meliola Fr.}

Hongsanan et al. (2015a) reported this genus has asexual morph which is directly produced from hyphae.

\section{Menispora Pers.}

Réblová et al. (2016a) proposed to adopt the older, asexually typified name Menispora rather than Zignoëlla Sacc. 1878, the younger, asexually typified name.

\section{Menisporopsis S. Hughes}

Réblová et al. (2016a) proposed to adopt Menisporopsis, the older, asexually typified name over Menisporopascus Matsush. 2003, the younger, sexually typified name.

\section{Metacapnodium Speg.}

Rossman et al. (2016) proposed to adopt Antennularia Rchb. 1828, Capnocybe S. Hughes, Capnophialophora S. Hughes 1966, Capnobotrys S. Hughes 1970, Capnosporium S. Hughes 1976 and Torulopsiella Bender 1932.

\section{Metarhizium Sorokīn}

Kepler et al. (2014) accepted that this genus has metacordyceps -like sexual morphs.

\section{Micraspis Darker}

Micraspis acicula the type species of Micraspis was introduced as the sexual morph of Periperidium acicola, the type species of Periperidium by Darker (1963) in the same publication. Hence, Johnston et al. (2014) proposed to adopt the sexually typified name which was introduced earlier.

\section{Microcyclus Sacc. et al.}

Kirk et al. (2008) mentioned that this genus has aposphaeria-like, fusicladium-like asexual morphs and tentatively placed in Mycosphaerellaceae. Monkai et al. (2013) also followed this placement.

Microdochium Syd. \& P. Syd.

Réblová et al. (2016a) proposed to adopt asexually typified name Microdochium 1924 rather than Monographella Petr. later in 1924, the sexually typified name. Both names were introduced in 1924 but Microdochium is widely used. 


\section{Microthecium Corda}

This genus is reported with an asexual morph which has phialidic conidiogenesis and hyaline conidia (Marin-Felix et al. 2018).

Microthia Gryzenh. \& M.J. Wingf.

Gryzenhout et al. (2006) introduced this genus with a coelomycetous asexual morph.

Microxiphium (Harv. ex Berk. \& Desm.) Thüm.

Rossman et al. (2016) proposed to adopt the older, sexually typified name rather than Dennisiella Bat. \& Cif. 1962, the younger, asexually typified name.

\section{Miladina Svrček}

Healy et al. (2016) adopted Miladina, the older, sexually typified name over Actinosporella Descals et al. 1999, the younger asexually typified name.

Moelleriella Bres.

Chaverri et al. (2008) reported an aschersonia-like asexual morph from this genus.

\section{Mollisia (Fr.) P. Karst.}

Kirk et al. (2008) reported Anguillospora, Casaresia, phialophora-like, Sirocyphellina asexual morphs from this genus. Seifert et al. (2011) also mentioned that Helicodendron Peyronel, Cadophora Lagerb. \& Melin, Cystodendron Bubak are also linked to Mollisia. However, these links have not been proved by phylogenetic analyses.

\section{Monascus Tiegh.}

Houbraken et al. (2020) accepted Basipetospora as the asexual morph of this genus.

\section{Monilinia Honey}

Johnston et al. (2014) proposed to adopt the younger, sexually typified name over Monilia Bonord. 1851, the older, asexually typified generic name.

\section{Monilochaetes Halst. ex Harter}

Réblová et al. (2016a) proposed to adopt the older, asexually typified name Monilochaetes, rather than Australiasca Sivan. \& Alcorn 2002.

\section{Monoblastia Riddle}

Hyde et al. (2013) and Hongsanan et al. (2020b) reported conidiomata (but rare) from the genus.

\section{Monochaetiellopsis B. Sutton \& DiCosmo}

Johnston et al. (2014) proposed to adopt Monochaetiellopsis, the younger, asexually typified name over Hypnotheca Tommerup 1970, the older, sexually typified name.

Morchella Dill. ex Pers.

Healy et al. (2016) adopted Desmazierella, the older, sexually typified name over Geniculodendron G.A. Salt 1974, younger, asexually typified name.

Muellerella Müll. Arg.

Muggia et al. (2015) assumed that this genus could be the sexual morph of Lichenodiplis.

Murichromolaenicola Mapook \& K.D. Hyde

Mapook et al. (2020) introduced this holomorphic genus with a hyphomycetous asexual 
morph.

Muriformistrickeria Q. Tian et al.

Tian et al. (2015) introduced this genus with a coelomycetous asexual morph. Phylogenetic analyses confirmed its familial placement in Melanommataceae

Murilentithecium Wanas. et al.

This genus with was introduced as a holomorphic genus by Wanasinghe et al. (2014). camarosporium-like asexual morph was observed from the culture.

\section{Murinectria M. Niranjan \& V.V. Sarma}

Hyde et al. (2020b) introduced this genus with a hyphomycetous asexual morph.

Muriphaeosphaeria Phukhams. et al.

This holomorphic genus with a coelomycetous asexual morph was introduced by Phukhamsakda et al. (2015). Phylogenetic analyses confirmed its placement in Phaeosphaeriaceae.

Murispora Y. Zhang bis et al.

Wanasinghe et al. (2015) reported phoma-like asexual morphs from the putative culture of $M$. hawksworthii.

\section{Mycocalicium Vain. ex Reinke}

Kirk et al. (2008) reported a phialophora-like asexual morph from this genus.

\section{Mycosphaerangium Verkley}

Voglmayr et al. (2020) reported coelomycetous asexual morph from this genus and confirmed its placement in Cenangiaceae.

\section{Mycosphaerelloides Videira \& Crous}

Videira et al. (2016) introduced this holoophic genus with a hyphomycetous asexual morph.

\section{Myriogenospora G.F. Atk.}

White \& Glenn (1994) reported an ephelis-like asexual morph from this genus.

\section{Myrmecridium Arzanlou et al.}

Réblová et al. (2016c) introduced M. montsegurinum Réblová \& J. Fourn., the first sexual morph of this genus.

\section{Myxocyclus Riess}

Tanaka et al. (2005) showed Splanchnonema argus as the sexual morph of M. polycystis based on co-occurrence.

\section{Nalanthamala Subram.} morph.

Lombard et al. (2015) reported holomorph of this genus with a hyphomycetous asexual

\section{Nannizziopsis Currah}

Kirk et al. (2008) and Seifert et al. (2011) reported Chrysosporium as an asexual morph of this genus but Chrysosporium is well distinct genus (Wijayawardene et al. 2020). Hence, we assume this genus might have been reported with a chrysosporium-like asexual morph.

Nectria (Fr.) Fr. 
Rossman et al. (2013) propose to adopt the younger, sexually typified name, Nectria over Tubercularia Tode 1790, the older, asexually typified name.

\section{Nemania Gray}

Geniculosporium Chesters \& Greenh. 1964, the younger, asexually typified name was regarded as a synonym of the older, sexually typified name, Nemania.

Neoacanthostigma Boonmee et al.

Boonmee et al. (2014) introduced this genus with a hyphomycetous asexual morph.

\section{Neoascochyta Q. Chen \& L. Cai}

Chen et al. (2015) introduced this genus with a coelomycetous asexual morph.

\section{Neobarya Lowen}

According to Candoussau et al. (2007) this genus has acremonium-like, lecanicillium-like, paecilomyces-like, or calcarisporium-like asexual morphs.

\section{Neobulgaria Petr.}

Kirk et al. (2008) and Seifert et al. (2011) mentioned that this genus has myrioconium-like or phialophora-like asexual morphs respectively.

Neocamarosporium Crous \& M.J. Wingf.

Wanasinghe et al. (2017a) reported the sexual morph of this genus.

Neochlamydotubeufia Y.Z. Lu et al.

$\mathrm{Lu}$ et al. (2018) introduced this genus. Sexual morph of this genus resembles Chlamydotubeufia while asexual morph resembles Dictyospora (Lu et al. 2018).

\section{Neocordyceps Kobayasi}

White et al. (2003) reported a hymenostilbe-like asexual morph from this genus.

Neocryphonectria C.M. Tian et al.

Jiang et al. (2020a) introduced this genus with a coelomycetous asexual morph.

Neocucurbitaria Wanas. et al.

Wanasinghe et al. (2017b) introduced this genus with a coelomycetous asexual morph.

Neocurreya Thambug. \& K.D. Hyde

Wijayawardene et al. (2016) introduced Neocurreya acaciae (Crous \& M.J. Wingf.) Wijayaw. et al. (Basionym: Curreya acaciae Crous \& M.J. Wingf.), a coelomycetous taxon which clustered in Neocurreya sensu stricto. However, Jaklitsch et al. (2016) regarded Neocurreya as a synonym of Teichospora Fuckel, the older sexually typified name. Hongsanan et al. (2020a) and Wijayawardene et al. (2020) did not agree with this synonymy. In here, we accept Neocurreya as a distinct genus. (However, Neocurreya acaciae (Crous \& M.J. Wingf.) Wijayaw. et al. in invalid fide Art. F.5.1 (Shenzhen))

Neodeightonia C. Booth

This genus is holomorphic which shows a coelomycetous asexual morph (Phillips et al. 2013).

Neodevriesia Quaedvl. \& Crous

Crous et al. (2015c) reported a teratosphaeria-like asexual morph of this genus. 
Neodidymelliopsis Q. Chen \& L. Cai

This genus was introduced with a coelomycetous asexual morph by Chen et al. (2015).

Neoerysiphe U. Braun

This genus was reported with a hyphomycetous asexual morph (Heluta et al. 2010).

Neoeutypella M. Raza et al.

Phookamsak et al. (2019) introduced this genus with a hyphomycetous asexual morph.

Neofabraea H.S. Jacks.

Phlyctema vagabunda Desm., the type species of Phlyctema is the asexual morph of Neofabraea alba (E.J. Guthrie) Verkley (Verkley 1999). Hence, Johnston et al. (2014) proposed to adopt Neofabraea as 'it is well characterized phylogenetically (Abeln et al. 2000, de Jong et al. 2001), and includes a number of plant pathogens, it seems expedient to protect the name.'

Neofusicoccum Crous et al.

Crous et al. (2006) introduced this genus with a coelomycetous asexual morphs (fusicoccumlike and dichomera-like) and botryosphaeria-like sexual morph.

Neogymnomyces G.F. Orr

Doveri et al. (2011) introduced $N$. virgineus Doveri et al. with a chrysosporium-like asexual morph.

Neohelicascus W. Dong et al.

Dong et al. (2020) introduced this genus from aquatic habitat and reported its coelomycetous asexual morph.

\section{Neomelanconium Petr.}

Voglmayr et al. (2020) reported that this asexual typified genus has sexual morph and confirmed its placement in Cenangiaceae.

Neolamproconium Crous \& Akulov (2019).

This holomorphic genus with coelomycetous asexual morph was introduced by Crous et al.

Neonectria Wollenw.

This genus was reported with Cylindrocarpon Wollenw. asexual morph thus, Chaverri et al. (2011) proposed to adopt it over Cylindrocarpon.

Neoparodia Petr. \& Cif.

This genus was reported with chuppia-like and sarcinella-like asexual morphs (Kirk et al. 2008, Seifert et al. 2011).

Neophaeoappendicospora Crous \& M.J. Wingf.

Crous et al. (2019) introduced this genus with a coelomycetous asexual morph.

Neophaeosphaeria M.P.S. Câmara et al.

Crous et al. (2013) introduced $N$. agaves Crous \& Yáñez-Moral., a coelomycetous taxon which clustered in Neophaeosphaeria sensu stricto. Later, Hyde et al. (2018) introduced $N$. phragmiticola A. Karunarathna \& K.D. Hyde, a coelomycetous taxon.

Neophyllachora Dayar. \& K.D. Hyde 
Dayarathne et al. (2017) introduced this genus with a coelomycetous asexual morph.

Neophysalospora Crous \& M.J. Wingf.

This genus was introduced by Crous et al. (2014b) with a coelomycetous asexual morph and showed that it belongs to Clypeophysalosporaceae

Neoplatysporoides Crous \& M.J. Wingf.

Crous et al. (2015d) introduced this genus with a coelomycetous asexual morph but in this study, they listed this genus under Pleosporaceae. Later, Crous \& Groenewald (2017) showed that this genus belongs in Libertasomycetaceae.

Neopseudocercosporella Videira \& Crous morph.

Videira et al. (2016) introduced this holomorphic genus with a hyphomycetous asexual

Neoroussoella Jian K. Liu et al.

Liu et al. (2014) introduced this genus with a coelomycetous asexual morph.

Neostagonosporella C.L. Yang et al. morph.

Yang et al. (2019) introduced this holomorphic genus which showed a coelomycetous asexual

Niesslia Auersw.

Gams et al. (2019) revised the genus and accepted it with monocillium-like asexual morphs.

Nigelia Luangsa-ard

Luangsa-ard et al. (2017) introduced this genus with asexual morph which is characterized by 'verticillately or irregularly branched conidiophores and solitary conidiogenous cells or phialides with or without lateral necks'.

Nigrocornus Ryley \& Langdon

White et al. (2003) reported an asexual morph which 'bears simple, indeterminate conidiophores and holoblastic, obclavate, multiguttulate, aseptate conidia'.

Nigrograna Gruyter et al.

Nigrograna cangshanensis Z.L. Luo et al. was introduced by Tibpromma et al. (2017) which represents the sexual morph of this genus.

Nigrospora Zimm.

Réblová et al. (2016a) proposed to adopt the older, asexually typified name Nigrospora rather than Khuskia H.J. Huds. 1963, the younger, sexually typified name.

Nigrovothelium Lücking et al. morph.

Hongsanan et al. (2020b) reported that this genus occasionally shows pycnidial asexual

Nothoseptoria Crous \& Bulgakov

Crous et al. (2019) introduced this coelomycetous genus which is morphologically resembles Septoria sensu stricto. However, phylogenetically both genera are distinct but accommodated in Mycosphaerellaceae.

Obolarina Pouzar 1986 
Kirk et al. (2008) reported a rhinocladiella-like asexual morph from this genus.

Occultibambusa D.Q. Dai \& K.D. Hyde

Dai et al. (2017) introduced this holomorphic genus with a coelomycetous asexual morph.

\section{Ochroconis de Hoog \& Arx}

Samerpitak et al. (2014) reported the sexual morph of this genus.

\section{Ocotomyces H.C. Evans \& Minter}

The type species of Ocotomyces, O. parasiticus H.C. Evans \& Minter is the sexual morph of Uyucamyces parasiticus H.C. Evans \& Minter 1985, the type species of Uyucamyces. Johnston et al. (2014) proposed to adopt the wisely used sexually typified name, Ocotomyces over Uyucamyces.

Oculimacula Crous \& W. Gams

Crous et al. (2003) introduced this genus and at the same time introduced its asexual morph, Helgardia Crous \& W. Gams 2003. Johnston et al. (2014) proposed to adopt the sexually typified name, Oculimacula over Helgardia.

\section{Ohleria Fuckel.}

Jaklitsch \& Voglmayr (2016) reported asexual morphs resembling 'phoma-like, synanamorph presumably monodictys-like'.

Omnidemptus P.F. Cannon \& Alcorn

Cannon \& Alcorn (1994) introduced this genus and its type species was reported with an asexual morph, Mycoleptodiscus affinis Alcorn \& P.F. Cannon.

\section{Ophiocordyceps Petch}

Quandt et al. (2014) proposed to adopt the younger, sexually typified name, Ophiocordyceps over the older, asexually typified generic names, Sorosporella Sorokin 1888 and Hirsutella Pat. 1892 as the sexual name is widely used.

Ophiodiaporthe Y.M. Ju et al.

Fu et al. (2013) introduced this genus with a coelomycetous asexual morph.

\section{Ophiostoma Syd. et al.}

The asexual morph of this genus has been accepted with 'sporothrixlike or pesotum-like conidiogenous cells with ellipsoidal to cylindrical obovate, globose and aseptate conidia' (Hyde et al. 2020b).

\section{Orbilia Fr.}

Baral et al. (2017) proposed to adopt Orbilia, the older, sexually typified name over Dicranidion Harkn., the younger, asexually typified name.

Orromyces B. Sur et al.

Kirk et al. (2008) reported chrysosporium-like asexual morphs.

\section{Ovulinia F.A. Weiss}

Johnston et al. (2014) proposed to adopt the older, sexually typified name, Ovulinia over the younger asexually typified name, Ovulitis N.F. Buchw. 1970

Paecilomyces Bainier 
Houbraken \& Samson (2011) and Houbraken et al. (2020) confirmed that Byssochlamys Westling 1909 and Paecilomyces cluster as a monophyletic group. The older, asexually typified name i.e., Paecilomyces, was adopted by Houbraken \& Samson (2011).

\section{Pallidocercospora Crous}

Crous et al. (2012a) introduced this holomorphic genus with a hyphomycetous asexual morph.

Palmiascoma Phook. \& K.D. Hyde

Liu et al. (2015) introduced this genus with a coelomycetous asexual morph.

Parabambusicola Kaz. Tanaka \& K. Hiray.

Tanaka et al. (2015) introduced this holomorphic genus with a hyphomycetous (monodictyslike) asexual morph.

Paraboeremia Q. Chen \& L. Cai

This genus was introduced with a coelomycetous asexual morph (Chen et al. 2015).

\section{Paracollema Otálora \& Wedin}

Otálora et al. (2014) introduced this genus with a pycnidial stage but the name was invalidly published. Hence, Wedin et al. (2017) validated the generic name.

\section{Paraconiothyrium Verkley}

Crous et al. (2020b) introduced Paraconiothyrium iridis Crous \& Akulov which represents sexual morph.

Paracremonium L. Lombard \& Crous

Lombard et al. (2015) introduced this genus with a hyphomycetous asexual morph.

Parafenestella Jaklitsch \& Voglmayr

This genus was introduced as a holomorphic genus with a coelomycetous asexual morph by Jaklitsch et al. (2018).

\section{Paragaeumannomyces Matsush.}

Réblová et al. (2020b) reported hyphomycetous asexual morphs from this genus.

Paragnomonia Senan. \& K.D. Hyde

Senanayake et al. (2017b) introduced this genus with a coelomycetous asexual morph.

Paraisaria Samson \& B.L. Brady

Mongkolsamrit et al. (2020) reinstated this genus and reported hyphomycetous asexual morph.

Paraleptosphaeria Gruyter et al.

Gruyter et al. (2012) introduced this holomorphic genus which has leptosphaeria-like sexual morph and phoma-like asexual morph. Hongsanan et al. (2020a) showed that Acicuseptoria Quaedvl. et al. 2013 (Quaedvlieg et al. 2013) clustered in Paraleptosphaeria sensu stricto thus regarded former genus as a synonym of later genus. This synonomy was accepted in Wijayawardene et al. (2020).

Paranectriella (Henn. ex Sacc. \& D. Sacc.) Höhn. 1910

Wijayawardene et al. (2014a) proposed to adopt Araneomyces Höhn., over Paranectriella as 
these two genera were reported as asexual and sexual morphs of one taxon respectively (Sutton 1980). However, Rossman et al. (2015b) did not agree with this adoption and proposed to conserve Paranectriella.

Parapallidocercospora Videira et al.

Videira et al. (2017) introduced this holomorphic genus and confirmed its phylogenetic placement in Mycosphaerellaceae.

Paraphaeosphaeria O.E. Erikss.

Verkley et al. (2014) reported a parconiothyrium-like asexual morph from this genus.

Pararoussoella Wanas. et al.

Crous et al. (2019) introduced P. juglandicola Crous \& R.K. Schumach., a coelomycetous taxon which clustered with Pararoussoella sensu stricto in Roussoellaceae.

Parodiella Speg. 1880

Kirk et al. (2008) reported that Ascochytopsis is the asexual morph of this genus thus Hongsanan et al. (2020b) also tentatively accepted it. However, Ascochytopsis is lacking DNA sequences thus its relationship with Parodiella is doubtful. Nevertheless, we tentatively accept that Parodiella has coelomycetous asexual morph.

Penicillifer Emden

Lombard et al. (2015) reported the holomorph of this genus.

Penicilliopsis Solms et al.

Houbraken \& Samson (2011) and Houbraken et al. (2020) accepted this genus as a holomorph.

\section{Penicillium Link}

Houbraken \& Samson (2011) reported the holomorph of the genus and provided the synonymy of the genus.

Periantria Döbbeler \& P.G. Davison

Döbbeler \& Davison (2018) introduced this genus with an acremonium-like asexual morph.

\section{Periconia Tode}

Tanaka et al. (2015) introduced Periconia homothallica Kaz. Tanaka \& K. Hiray. and P. pseudodigitata Kaz. Tanaka \& K. Hiray., holomorphic taxa which grouped in Periconia sensu stricto.

\section{Perisporiopsis Henn.}

Kirk et al. (2008) reported that this genus has Septoidium asexual morph. However, based on early studies, Seifert et al. (2011) reported that Septoidium has been linked with several other sexual genera. Septoidium is lacking sequence data in the GenBank thus the sexual-asexual links among genera are doubtful. However, in here, we tentatively report hyphomycetous asexual morph from Perisporiopsis.

Petrakia Syd. \& P. Syd.

Butin et al. (2013) stated that Petrakia showed Mycodidymella sexual morphs. Hongsanan et al. (2020a) and Wijayawardene et al. (2020) listed this genus under Melanommataceae.

Pezicula Tul. \& C. Tul. 
Verkley et al. (1999) accepted Cryptosporiopsis fasciculata as the asexual morph of $P$. carpinea, the type species of Pezicula. Furthermore, Verkley (1999) regarded Pezicula ocellata as the sexual morph of $C$. nigra the type species of Cryptosporiopsis. Hence, Johnston et al. (2014) proposed to adopt Pezicula, older sexually typified name over Cryptosporiopsis, the younger asexually typified name.

\section{Phacidiopycnis Potebnia}

Johnston et al. (2014) proposed to adopt the older, asexually typified name over the younger, sexually typified name Potebniamyces Smerlis 1962.

\section{Phacidium Fr.}

Johnston et al. (2014) proposed to adopt the older, sexually typified name Phacidium, over Ceuthospora Grev. 1827, the younger, asexually typified name.

Phaeoacremonium W. Gams et al.

Réblová et al. (2015) proposed to adopt asexually typified name, Phaeoacremonium over sexually typified genera, Togninia Berl. 1900 and Romellia Berl. 1900. The species of Phaeoacremonium are known as pathogens of human and vascular plants.

Phaeobotryon Theiss. \& Syd.

Phillips et al. (2013) accepted this genus as a holomorphic genus.

\section{Phaeodimeriella Speg.}

Boonmee et al. (2017) reported a coelomycetous asexual morph from the holotype of the type species of this genus this genus.

Phaeopoacea Thambug. et al.

Thambugala et al. (2017b) introduced this genus with a coelomycetous asexual morph.

\section{Phaeosphaeria I. Miyake}

Hongsanan et al. (2020a) reported the asexual morphs of this genus as phoma-like and stagonospora-like based on previous studies.

Phaeosphaeriopsis M.P.S. Câmara et al.

Câmara et al. (2003) introduced this genus with a coniothyrium-like, coelomycetous asexual morph.

Phialocephala W.B. Kendr.

In phylogenetic analyses, Phaeomollisia T.N. Sieber \& Grünig resides in Phialocephala sensu stricto (Grunig et al. 2009, Day et al. 2012). Hence, Johnston et al. (2014) proposed to adopt Phialocephala, the older, asexually typified name over Phaeomollisia, the younger sexually typified name.

\section{Phoma Sacc.}

Chen et al. (2015) reported the holomorph of Phoma and provided the description.

\section{Phomatospora Sacc.}

Kirk et al. (2008) reported a sporothrix-like asexual morph from this genus.

\section{Phomatospora Sacc.}

Rappaz (1992) and Fournier \& Lechat 2010) reported sporothrix-like asexual morphs from this genus. 
Phragmocapnias Theiss. \& Syd.

Chomnunti et al. (2011) regarded the younger, asexul typified genus, Conidioxyphium Bat. \& Cif. 1963 as a synonym of this genus. Rossman et al. (2015b) agreed with this synonymy.

Phyllactinia Lév. 1851

Kirk et al. (2008), Seifert et al. (2011) listed Ovulariopsis Pat. \& Har. 1900 as the asexual morph of this genus.

Phyllobathelium (Müll. Arg.) Müll. Arg.

A coelomycetous asexual morph was reported in this lichenized genus by Hongsanan et al. (2020b).

\section{Phyllocharis Fée}

Hongsanan et al. (2020b) reported coelomycetous asexual morph from this lichenized genus.

\section{Phylloporis Clem.} (2020b).

A coelomycetous asexual morph was reported in this lichenized genus by Hongsanan et al.

\section{Phyllosticta Pers.}

Wikee et al. (2011), Wijayawardene et al. (2014a) and Rossman et al. (2015b) proposed to accept Phyllosticta, the older, asexually typified name over Guignardia the younger, sexually typified name.

\section{Pilidium Kunze 1823}

Johnston et al. (2014) proposed to adopt the older, asexually typified name, Pilidium over the younger, sexually typified name, Discohainesia Nannf. 1932.

\section{Plenodomus Preuss}

This genus represents a holomorphic taxon which has leptosphaeria-like sexual morph and phoma-like asexual morph.

\section{Pleochaeta Sacc. \& Speg.} morph

Seifert et al. (2011) stated that this genus has Streptopodium R.Y Zheng \& G.Q. Chen asexual

\section{Pleoseptum A.W. Ramaley \& M.E. Barr}

Ramaley \& Barr (1995) introduced this genus with P. yuccaesedum A.W. Ramaley \& M.E. Barr as the type species. At the same time, Ramaley \& Barr (1995) reported Camarosporium yuccaesedum as the asexual morph of $P$. yuccaesedum. However, Camarosporium sensu stricto is in Camarosporiaceae thus Wijayawardene et al. (2016) regarded that Pleoseptum has camarosporium-like asexual morph.

Pleurostoma Tul. \& C. Tul.

Réblová et al. (2016a) proposed to adopt, the older, sexually typified name, Pleurostoma rather than Pleurostomophora Vijaykr. et al. 2004, the younger, asexually typified name.

\section{Pleurotheciella Réblová et al.}

Réblová et al. (2012) introduced this genus with a dactylaria-like asexual morph.

\section{Pleurothecium Höhn.}

Réblová et al. (2016a) proposed to adopt Pleurothecium, the older, asexually typified name 
rather than Carpoligna F.A. Fernández \& Huhndorf 1999, the younger, sexually typified name.

Ploioderma Darker.

Johnston et al. (2014) proposed to adopt Ploioderma, the older, sexually typified name over Sphaeronaema Fr. 1815, the older, asexually typified name.

Plurivorosphaerella O. Hassan \& T.H. Chang

Hassan \& Chang (2019) introduced this genus to accommodate Mycosphaerella nawae Hiura \& Ikata which is not congeneric with Mcosphaerella sensu stricto. The type species of Plurivorosphaerella, P. nawae (Hiura \& Ikata) O. Hassan \& T. Chang has hyphomycetous asexual morph thus the genus represents holomorph.

Poaceicola W.J. Li et al.

This genus was introduced with a coelomycetous asexual morph by $\mathrm{Li}$ et al. (2015). In phylogenetic analyses, Li et al. (2015) showed that Poaceicola resides in Phaeosphaeriaceae.

Pochonia Bat. et al.

Zare et al. (2001) reported Metacordyceps sexual morph (as Cordyceps) from this genus.

\section{Podonectria Petch}

Boonmee et al. (2014) reported a hyphomycetous asexual morph from this genus.

\section{Podosphaera Kunze}

Kirk et al. (2008) stated that Oidium subgen. Fibroidium is the asexual morph of this genus.

\section{Podospora Ces. 1856}

A phialophora-like asexual morph was reported from this genus (Seifert et al. 2011).

\section{Polycephalomyces Kobayasi}

Blistum B. Sutton 1973, the younger, asexually typified name was regarded as a synonym of this generic name (Quandt et al. 2014).

Polymeridium (Müll. Arg.) R.C. Harris

Hongsanan et al. (2020b) reported that this genus shows pycnidial asexual morphs.

Polyplosphaeria Kaz. Tanaka \& K. Hiray.

Li et al. (2016) introduced P. thailandica C.G. Lin et al., a hyphomycetous taxon and confirmed that it belongs in Polyplosphaeria sensu stricto.

Populocrescentia Wanas. et al.

Wanasinghe et al. (2018) amended the genus to include hyphomycetous asexual morphs. Populocrescentia rosae Wanas. et al. represents a hyphomycetous asexual morph.

\section{Poronia Willd.} culture.

Ju \& Rogers (2001) reported the asexual morph of Poronia pileiformis (Berk.) Fr. from the

\section{Praetumpfia Jaklitsch \& Voglmayr}

Jaklitsch \& Voglmayr (2017) introduced this holomorphic genus with a coelomycetous asexual morph.

Pragmopora A. Massal 
Johnston et al. (2014) proposed to adopt the older, sexually typified name, Pragmopora over the younger, asexually typified name, Pragmopycnis B. Sutton \& A. Funk 1975.

\section{Prillieuxina G. Arnaud}

Hongsanan et al. (2014) proposed to reduce the asexually typified name Leprieurina G. Arnaud under Prillieuxina. Subsequent studies by Wijayawardene et al. (2014a) and Rossman et al. (2015b) followed this synonymy.

\section{Prosthemium Kunze}

Wijayawardene et al. (2014a) and Rossman et al. (2015b) proposed to adopt the older, asexually typified name Prosthemium over Pleomassaria Speg.

\section{Protocrea Petch}

Jaklitsch \& Samuels (2008) reported a gliocladium-like species as asexual morph of this genus.

\section{Protocreopsis Yoshim}

Seifert et al. (2011) reported an acremonium-like asexual morph from this genus.

Protofenestella Jaklitsch \& Voglmayr

This holomorphic genus was introduced by Jaklitsch et al. (2018) and confirmed its familial placement in Cucurbitariaceae.

\section{Protoventuria Berl. \& Sacc.}

Kirk et al. (2008) reported a fusicladium-like asexual morph from this genus.

Pruniphilomyces Crous \& Bulgakov

Crous et al. (2019) introduced this holomorphic genus with hyphomycetous asexual morph.

Pseudoasteromassaria M. Matsum. \& Kaz. Tanaka

Ariyawansa et al. (2015) introduced this genus with a coelomycetous asexual morph.

Pseudoastrosphaeriella Phook. et al.

Phookamsak et al. (2015) introduced this genus with a coelomycetous asexual morph.

Pseudobogoriella Lücking et al.

Hongsanan et al. (2020b) introduced this genus with a pycnidial asexual morph.

Pseudocoleophoma Kaz. Tanaka \& K. Hiray.

Tanaka et al. (2015) introduced this genus with a coelomycetous asexual morph.

Pseudocosmospora C. Herrera \& P. Chaverri

Herrera et al. (2013) introduced this genus with a hyphomycetous asexual morph (acremonium-like to verticillium-like).

Pseudocryptoclypeus A. Hashim. \& Kaz.

This genus was introduced by Hashimoto et al. (2017) with a coelomycetous asexual morph. Phylogenetic analyses showed that it belongs in Lophiotremataceae.

\section{Pseudodidymella C.Z. Wei et al.}

This genus was reported with a 'pycnopleiospora-like asexual morph and produce mycopappus-like propagules in their asexual morphs' (Hongsanan et al. 2020a). 
Pseudodidymosphaeria Thambug. \& K.D. Hyde (2015a).

This genus was introduced with a coelomycetous asexual morph by Thambugala et al.

Pseudomonodictys Doilom et al.

Hongsanan et al. (2020a) and Wijayawardene et al. (2020) listed this genus under Parabambusicolaceae.

Pseudoornatispora Tibpromma \& K.D. Hyde

Tibpromma et al. (2018b) introduced this genus with a hyphomycetous asexual morph.

Pseudopaucispora A. Hashim. 2018

This genus was introduced by Hashimoto et al. (2018) with a coelomycetous asexual morph. However, the authors mentioned the conidiomata as pseudopycnidial.

\section{Pseudopezicula Korf}

Korf et al. (1986) introduced this genus with a phialophora-like asexual morph.

Pseudoplagiostoma Cheew. et al.

Cheewangkoon et al. (2010) introduced this genus with a coelomycetous asexual morph.

Pseudopyrenula Müll. Arg. morph.

Hongsanan et al. (2020b) reported that this genus occasionally shows pycnidial asexual

Pseudoroussoella Mapook \& K.D. Hyde

Mapook et al. (2020) introduced this genus with coniothyrium-like asexual morphs.

Pseudoscopulariopsis M. Sandoval-Denis et al.

Sandoval-Denis et al. (2016) introduced this genus with a hyphomycetous asexual morph.

\section{Pseudosolidum Lloyd}

Kirk et al. (2008) reported a plectophomella-like asexual morph.

Pseudospiromastix Guarro et al.

Hirooka et al. (2016) introduced this genus with a hyphomycetous asexual morph.

\section{Pseudosplanchnonema Chethana et al.}

Chethana et al. (2015) introduced this holomorphic genus with a coelomycetous (phoma-like) asexual morph.

\section{Pseudothiella Petr.}

Réblová et al. (2016a) proposed to adopt Pseudothiella over Pseudothiopsella Petr. since the former sexually typified name has been used more than the latter. Both names were introduced at the same time by Petrak (1928) to represent the sexual and asexual morphs of one taxon.

\section{Pseudothiella Petr.}

This older, sexually typified name was proposed to accept rather than Pseudothiopsella Petr. 1928 , the younger asexually typified name.

\section{Pseudozasmidium Videira \& Crous}

Videira et al. (2017) introduced this holomorphic genus with a coelomycetous asexual morph. 
Asexual morph morphologically resembles Zasmidium sensu stricto.

Puiggariella Speg.

Hongsanan et al. (2020b) reported a coelomycetous asexual morph from this lichenized genus.

Purpurepithecium Jayasiri \& K.D. Hyde

Jayasiri et al. (2017) introduced this genus with a hyphomycetous asexual morphs.

\section{Puttemansia Henn.}

Kirk et al. (2008) reported that Guelichia, Tetracrium are asexual morphs of this genus. However, subsequent publications by Wijayawardene et al. (2014a), Rossman et al. (2015b, 2016) did not consider this connection. Seifert et al. (2011) reported that Spermatoloncha, Tetracrium and Titaea were linked with Puttemansia. Type species of Guelichia, Puttemansia, Spermatoloncha Tetracrium and Titaea are lacking DNA sequences thus their relationships cannot be confirmed. However, we tentatively accept that Puttemansia has hyphomycetous asexual morph since two major publications reported it (Kirk et al. 2008, Seifert et al. 2011).

Pycnopeziza W.L. White \& Whetzel

Johnston et al. (2014) proposed to adopt Pycnopeziza over the older, asexually typified name, Acarosporium Bubák \& Vleugel ex Bubák 1911 (= Chaetalysis Peyronel 1922; Ciliosira Syd. 1942).

Pyrenopeziza Fuckel

Johnston et al. (2014) proposed to adopt the sexually typified name Pyrenopeziza over Cylindrosporium Grev.

\section{Pyrenophora Fr.}

Wijayawardene et al. (2014a) proposed to adopt this genus over Drechslera S. Ito (Art. F.8). Rossman et al. (2015b) agreed with Wijayawardene et al. (2014a) and proposed to reduce Marielliottia Shoemaker under Pyrenophora.

\section{Raciborskiella Höhnel} genus.

Hongsanan et al. (2020b) reported a coelomycetous asexual morph from this lichenized

\section{Racoplaca Fée}

Hongsanan et al. (2020b) reported a coelomycetous asexual morph from this lichenized genus.

\section{Ragnhildiana Solheim}

This genus was regarded as a synonym of Mycovellosiella by Muntañola (1960). However, Crous \& Braun (2003) treated Ragnhildiana and Mycovellosiella as synonyms of Passalora. Nevertheless, Videira et al. (2017) showed that Ragnhildiana phylogenetically distinct from Mycovellosiella and Passalora. Hence, Ragnhildiana was reinstated and accepted as a genus in Mycosphaerellaceae.

\section{Ramularia Unger}

Wijayawardene et al. (2014a) and Rossman et al. (2015b) proposed to adopt the older, asexually typified name, Ramularia over Mycosphaerella Johanson 1884, the younger, sexually typified name. 
Rasamsonia Houbraken et al.

Houbraken et al. (2011) introduced this genus with a hyphomycetous morph.

Readeriella Syd. \& P. Syd.

Crous et al. (2009) reported this genus with a teratosphaeria-like sexual morph and Cibiessia synasexual morph.

Rectifusarium L. Lombard et al.

This genus was introduced by Lombard et al. (2015) with a hyphomycetous asexual morph.

Regiocrella Chaverri et al.

Chaverri et al. (2005) introduced this genus with sphacelia-like asexual morph.

Remotididymella Valenzuela-Lopez et al.

Jayasiri et al. (2019) introduced $R$. bauhiniae Jayasiri et al., the first taxon which represents the sexual morph of this genus.

Rhabdocline Syd. 1922

Sydow \& Petrak (1922) introduced Rhabdocline and Rhabdogloeum in the same publication but former is described in page number 194 while the latter in 215. Parker \& Reid (1969) mentioned that the type species of both genera have been linked. Johnston et al. (2014) proposed to adopt Rhabdocline over Rhabdogloeum Syd. since former is older and widely used.

Rhachisphaerella U. Braun et al.

Videira et al. (2017) introduced this holomorphic genus with a hyphomycetous asexual morph.

\section{Rhamphoria Niessl}

Réblová \& Štepánek (2018) reported a phaeoisaria-like asexual morph and an idriella-like synanamorph from in vitro conditions in two species of Rhamphoria.

\section{Rhizothyrium Naumov}

Johnston et al. (2014) proposed to adopt Rhizothyrium, the older, asexually typified name over Rhizocalyx Petr. 1928, the younger, sexually typified name.

Rhopalostroma D. Hawksw.

Kirk et al. (2008) reported a nodulisporium-like asexual morph from this genus.

\section{Rhytisma Fr.}

Johnston et al. (2014) proposed to adopt Rhytisma, the older, sexually typified name over Melasmia Lév. 1846, the younger, asexually typified name.

Rosellinia de Not.

Réblová et al. (2016a) adopted the older, sexually typified name Rosellinia over Dematophora R. Hartig 1883, the younger, asexually typified name.

Rostrohypoxylon J. Fourn. \& M. Stadler

Fournier et al. (2010) introduced this genus with a nodulisporium-like asexual morph.

\section{Roumegueriella Speg.}

Kirk et al. (2018) reported a gliocladium-like asexual morph while Seifert et al. (2011) linked it with Acremonium. 


\section{Roussoella Sacc.}

This genus was accepted with a coelomycetous asexual morphs (cytoplea-like) by Liu et al. (2014), Dai et al. (2017) and Wijayawardene et al. (2016).

Roussoellopsis I. Hino \& Katum.

Liu et al. (2014) accepted that this genus belongs in Roussoellaceae and reported that Roussoellopsis macrospora has melanconium-like asexual morphs.

Rubroshiraia D.Q. Dai \& K.D. Hyde

Dai et al. (2019) introduced this genus with a coelomycetous asexual morph.

\section{Rutstroemia P. Karst.}

Kirk et al. (2008) reported a phialophora-like asexual morph from this genus.

Ruwenzoria J. Fourn. et al.

Stadler et al. (2010) introduced this genus with nodulosporium-like or sporothrix-like asexual morphs.

\section{Ruzenia O. Hilber}

Miller \& Huhndorf (2004) introduced this genus with a selenosporella-like asexual morph.

\section{Saccharata Denman \& Crous} morph.

Slippers et al. (2013) reported the holomorphism of this genus with a coelomycetous asexual

\section{Saccothecium Fr.}

Kirk et al. (2008) reported an aureobasidium-like asexual morph from this genus.

Samsoniella Mongkols. et al.

Mongkolsamrit et al. (2018) introduced this genus with isaria-like asexual morphs.

\section{Samuelsia Chaverri \& K.T. Hodge}

Chaverri et al. (2008) introduced this genus with an aschersonia-like asexual morph.

\section{Sarcinella Sacc.}

Rossman et al. (2016) proposed to adopt Sarcinella over Schiffnerula Höhn. 1909 (Art. F.8). However, Sarcinella lacks DNA sequences thus its phylogenetic placement is uncertain. Nevertheless, we tentatively follow Rossman et al. (2016).

\section{Sarcoscypha (Fr.)}

Boud. Healy et al. (2016) adopted Sarcoscypha, older, sexually typified name over Molliardiomyces Paden 1984, younger asexually typified name.

\section{Sawadaea Miyabe}

Kirk et al. (2008) stated that Oidium subgen. Octagoidium is the asexual morph of this genus.

Sclerenchymomyces Phukhams. \& K.D. Hyde

Phukhamsakda et al. (2020) introduced this genus with a coelomycetous asexual morph.

\section{Sclerocleista Subram.}


Houbraken \& Samson (2011) stated that 'Sclerocleista differs from Penicillium sensu stricto. in having an Aspergillus-type anamorph and purple coloured cleistothecia flled with lenticular ascospores'.

\section{Scleropezicula Verkley}

Johnston et al. (2014) proposed to adopt sexually typified name Scleropezicula over Cryptosympodula Verkley.

\section{Scytalidium Pesante}

Johnston et al. (2014) proposed to adopt this generic name over the younger, sexually typified name, Xylogone Arx \& T. Nilsson 1969.

\section{Seaverinia Whetzel}

Johnston et al. (2014) proposed to adopt Seaverinia, the older, sexually typified name over Verrucobotrys Hennebert 1973, the younger, asexually typified name.

\section{Seimatosporium Corda}

Réblová et al. (2016a) proposed to adopt older, asexually typified name Seimatosporium 1833 rather than Discostroma Clem. 1909, the younger, sexually typified name.

Septotinia Whetzel ex J.W. Groves \& M.E. Elliott

Johnston et al. (2014) proposed to adopt Septotinia, older sexually typified name over Septotis N.F. Buchw. ex Arx 1970, the younger, asexually typified name.

Seriascoma Phook. Hyde

Dai et al. (2017) introduced this holomorphic genus with a coelomycetous asexual morph.

\section{Serusiauxiella S.H. Jiang et al.}

Jiang et al. (2020b) introduced this genus with a coelomycetous asexual morph.

\section{Setophaeosphaeria Crous \& Y. Zhang ter}

Crous et al. (2014a) introduced this genus with a coelomycetous asexual morph and accepted as a genus in Phaeosphaeriaceae. However, subsequent study by Phookamsak et al. (2014) showed that Setophaeosphaeria did not group in Phaeosphaeriaceae thus regarded as a genus in Pleosporales, genera incertae sedis.

\section{Shiraia Henn.}

This genus was reported with a coelomycetous asexual morph which has 'holoblastic conidiogenous cells bearing asymmetrical, hyaline to light brown, fusiform, muriform conidia with acute basal, obtuse apex, or both obtuse ends' (Hyde et al. 2013, Hongsanan et al. 2020a)

\section{Sodiomyces A.A. Grum-Grzhim. et al.}

Grum-Grzhimaylo et al. (2013) introduced this genus with hyphomycetous asexual morph but was invalid. Hence, Crous et al. (2019)

\section{Spadicoides S. Hughes}

Réblová et al. (2018) reported the sexual morph of S. bina, S. fuscolutea and S. hyalostoma. Moreover, Réblová et al. (2018) reported a selenosporella-like synasexual morph of S. bina and $S$. fuscolutea in vitro.

Sparticola Phukhams. et al.

This genus was introduced by Phukhamsakda et al. (2016) with a hyphomycetous asexual 
morph and showed that it belongs in Sporormiaceae.

Sphaeronaemella P. Karst.

Réblová et al. (2016a) proposed to adopt this sexually typified name rather than Gabarnaudia Samson \& W. gams 1974, the younger, asexually typified name.

Sphaeropsis Sacc.

Phillips et al. (2013) accepted this genus as a holomorphic genus with a coelomycetous asexual morph.

Sphaerostilbella (Henn.) Sacc. et al.

Rossman et al. (2013) proposed to adopt the younger, widely-used, sexually typified name, Sphaerostilbella, over the older, asexually typified name Gliocladium corda 1840.

Spiromastigoides Doweld (= Spiromastix Kuehn \& G.F. Orr)

Hirooka et al. (2016) introduced six Spiromastix species with hyphomycetous asexual morphs.

\section{Sporidesmium Link} morph.

Zhang et al. (2017) introduced S. thailandense W. Dong et al., which represents a sexual

Sporophagomyces K. Põldmaa et al.

Põldmaa et al. (2000) reported an acremonium-like asexual morph from this genus.

Sporoschisma Berk. \& Broome

Réblová et al. (2016a) proposed to adopt this older, asexually typified name rather than Melanochaeta E. Müll. et al. 1969, the younger, sexually typified name.

Sporoschismopsis Hol-Jech. et al.

Réblová et al. (2016a) proposed to adopt Sporoschismopsis, the older, asexually typified name rather than Porosphaerellopsis Samuels \& E. Müll. 1982, the younger sexually typified name.

Sporothrix Hektoen et al.

de Beer et al. (2016) proposed to reduce Sporotrichopsis Gueguen. and Dolichoascus Thibaut $\&$ Ansel. under Sporothrix

Spumatoria Massee \& E.S. Salmon

Giraldo et al. (2017) reported a sporothrix-like asexual morph from the culture.

\section{Stachybotrys Corda}

Wang et al. (2015) showed that Stachybotrys sensu stricto comprises Ornatispora K.D. Hyde et al. thus proposed to adopt the older, asexually typified name, Stachybotrys over the younger sexually typified name. Lombard et al. (2016) regarded Ornatispora as a synonym of Stachybotrys and provided the description of the holomorph.

Stagonosporopsis Died.

Aveskamp et al. (2010) and Chen et al. (2015) reported the holomorph of this genus and provided the description.

Stamnaria Fuckel 
Johnston et al. (2014) proposed to adopt Stamnaria, the older, sexually typified name over Titaeospora Bubák 1916, the younger, asexually typified name.

Stanjehughesia Subram.

Réblová et al. (2016a) proposed to adopt the older, asexually typified name Stanjehughesia rather than Umbinosphaeria Réblová 1999, the younger, sexually typified name.

Staurosphaeria Rabenh.

Wanasinghe et al. (2017a) showed that Hazslinszkyomyces Crous \& R.K. Schumach. grouped in Staurosphaeria sensu stricto. Hence, Wanasinghe et al. (2017a) regarded that Staurosphaeria has Hazslinszkyomyces asexual morphs and latter was reduced under former.

\section{Stegonsporium Corda} 2020b).

This genus has a prosthecium-like sexual morph (Senanayake et al. 2017a, Hyde et al.

\section{Stemphylium Wallr.}

Wijayawardene et al. (2014a) proposed to adopt Stemphylium over Pleospora. This synonymy was accepted by Rossman et al. (2015b).

Stictis Pers.

Ciferrit (1957) introduced Stictospora Cif. to accommodate the conidial stage of Stictis.

\section{Stictophacidium Rehm}

Kirk et al. (2008) reported 'Ebollia, coelophoma-like' asexual morph from this genus. However, Minter \& Caine (1980) did not mention about the sexual morph of Ebollia, when they introduced it.

Stigmatodiscus Voglmayr \& Jaklitsch

Voglmayr et al. (2016) introduced this genus with a coelomycetous asexual morph. Hongsanan et al. (2020b) regarded Asterodiscus Voglmayr et al. as a synonym of this genus.

\section{Stilbospora Pers.}

Rossman et al. (2015a) proposed to adopt the older, asexually typified name Stilbospora rather than Prosthecium Fresen. 1852, the younger, sexually typified name.

\section{Stomiopeltis Theiss.}

Kirk et al. (2008) reported Sirothyriella Höhn. as the asexual morph of this genus, thus Index Fungorum (2021) listed Sirothyriella as a synonym of Stomiopeltis. Akaropeltella M.L. Fan 1972 is also regarded as a latter synonym of Stomiopeltis. Kirk et al. (2008) reported sporidesmium-like asexual morph from Akaropeltella.

\section{Streptotinia Whetzel}

Johnston et al. (2014) proposed to adopt Streptotinia, the older, sexually typified name over Streptobotrys Hennebert 1973, the younger, asexually typified name.

Striatibotrys L. Lombard \& Crous

Lombard et al. (2016) introduced this genus with a hyphomycetous asexual morph.

Striatosphaeria Samuels \& E. Müll.

Réblová et al. (2020b) introduced Striatosphaeria castanea Réblová \& J. Fourn. which produced hyphomycetous asexual morph from the culture. 


\section{Strossmayeria Schulzer}

Johnston et al. (2014) proposed to adopt Strossmayeria, the older, sexually typified name over Pseudospiropes M.B. Ellis 1971, the younger asexually typified name.

\section{Strigula Fr.}

Hongsanan et al. (2020b) reported a coelomycetous asexual morph for this genus.

\section{Subbaromyces Hesselt.}

This genus was reported with both the sexual and asexual morphs (Hesseltine 1953).

\section{Sulcatispora Kaz.}

Tanaka \& K. Hiray. This genus was introduced with a coelomycetous asexual morph and confirmed its familial placement in Sulcatisporaceae by Tanaka et al. (2015).

Swinscowia S.H. Jiang

Hongsanan et al. (2020b) introduced this holomorphic lichenized genus with coelomycetous asexual morph.

\section{Symphyosirinia E.A. Ellis}

Johnston et al. (2014) proposed to adopt this generic name over the younger, asexually typified name, Symphyosirella Seifert 2009.

\section{Sympoventuria Crous \& Seifert}

Crous et al. (2007b) introduced this genus with a sympodiella-like asexual morph.

\section{Syncarpella Theiss. \& Syd.}

Ramaley \& Barr (1997) introduced Syntholus A.W. Ramaley \& M.E. Barr 1997 as the asexual morph of Syncarpella ribis A.W. Ramaley \& M.E. Barr. Hence, the younger, asexually typified generic name, Syntholus has been regarded as a synonym of Syncarpella (Index Fungorum 2021).

\section{Synfenestella Jaklitsch \& Voglmayr}

Jaklitsch \& Voglmayr (2019) introduced this holomorphic genus with a coelomycetous asexual morph.

\section{Synnemasporella X.L. Fan \& J.D.P. Bezerra}

Fan et al. (2018) introduced this genus with two types of asexual morphs (coelomycetous and hyphomycetous). Both morphs have been reported from the host plant.

\section{Talaromyces C.R. Benj.}

This genus was reported with penicillium-like asexual morph (Houbraken \& Samson 2011, Yilmaz et al. 2014, Houbraken et al. 2020).

Tamaricicola Thambug. et al.

Thambugala et al. (2017a) introduced this genus with a phoma-like coelomycetous asexual morph.

\section{Teichospora Fuckel}

Jaklitsch et al. (2016) reported coelomycetous morph with 'brown unicellular (coniothyriumlike), rarely hyaline unicellular (aposphaeria-like) or several celled brown conidia' from this genus.

Tenuiappendicula Senan. et al. 
Senanayake et al. (2017b) introduced this genus with a coelomycetous asexual morph.

Tenuitholiascus S.H. Jiang et al.

Jiang et al. (2020c) introduced this genus with a coelomycetous asexual morph.

Teracosphaeria Réblová \& Seifert

Réblová \& Seifert (2007) introduced this genus with a phialophora-like asexual morph.

Teratosphaeria Syd. \& P. Syd.

Crous et al. (2009) confirmed that Kirramyces J. Walker et al. 1992 and Colletogloeopsis Crous \& M.J. Wingf. 1997 are congeneric with Teratosphaeria. Hence, regarded the both asexual morphs as synonyms of Teratosphaeria. Moreover, Teratosphaeria was reported with batcheloromyces-like synasexual morphs.

Tetraploa Berk. \& Broome (= Tetraplosphaeria Kaz. Tanaka \& K. Hirayama)

Tanaka et al. (2009) introduced sexual morph of this genus as Tetraplosphaeria. Wijayawardene et al. (2014a) proposed to adopt older asexually typified name (i.e., Tetraploa) over Tetraplosphaeria.

Thailandiomyces Pinruan et al.

Pinruan et al. (2008) introduced this genus and stated that the type species of this genus, $T$. bisetulosus Pinruan et al. has Craspedodidymum licualae asexual morph.

\section{Thaxteriellopsis Sivan.}

Doilom et al. (2017) reported the asexual morph of T. lignicola.

\section{Thermoascus Miehe}

Houbraken \& Samson (2011) mentioned that the asexual morph of this genus can be similar to Paecilomyces.

Thermothelomyces Y. Marín et al.

Marin-Felix et al. (2015) introduced tis genus with a hyphomycetous asexual morph.

\section{Thyridium Nitschke}

Leuchtmann \& Müller (1986) reported a coelomycetous asexual morph, or produce holoblastic sympodial conidia from hyphae.

\section{Thyronectria Sacc.} morph.

Lombard et al. (2015) reported holomorph of this genus with a hyphomycetous asexual

Tirisporella E.B.G. Jones et al.

Tirisporella beccariana (Ces.) E.B.G. Jones et al., the type species of Tirisporella has been reported with Phialophora cf. olivaceae asexual morph. (Suetrong et al. 2015).

Togniniella Réblová et al.

Réblová et al. (2004) introduced this genus with its hyphomycetous asexual morph Phaeocrella. Réblová et al. (2016a) proposed to adopt Togniniella over Phaeocrella.

\section{Tolypocladium W. Gams}

Quandt et al. (2014) proposed to adopt the older, sexually typified name, Tolypocladium over younger, asexually typified name Chaunopycnis W. Gams 1980. 


\section{Torpedospora Meyers}

Réblová et al. (2016a) proposed to adopt Torpedospora rather than Glomerulispora AbdelWahab \& Nagah. 2010, the younger, asexually typified name.

Towyspora Wanas. et al.

This genus was introduced by Li et al. (2016) with a coelomycetous asexual morph and phylogenetic analyses showed that it belongs to Lentitheciaceae.

Tremateia Kohlm. et al.

Kohlmeyer et al. (1995) introduced this genus with a phoma-like asexual morph.

\section{Trematosphaeria Fuckel}

Zalerion-like asexual morphs have been reported by Kirk et al. (2008) and Hyde et al. (2013) reported a hyphopodia-like structures from the cultures of this genus.

Tricharina Eckblad

Kirk et al. (2008) reported that Ascorhizoctonia Chin S. Yang \& Korf 1985 as the asexual morph of Tricharina. Hence, Wijayawardene et al. (2020) regarded Ascorhizoctonia as a synonym of Tricharina.

\section{Trichoderma Pers.}

Rossman et al. (2013) proposed to adopt the older, asexually typified name, Trichoderma rather than Hypocrea Fr. 1825, the younger, asexually typified name.

\section{Trichomerium Speg.}

Kirk et al. (2008) stated that Tripospermum Speg. 1918 as the asexual morph. However, this link was not proved and Tripospermum was accepted as a genus in Neodevriesiaceae by Wijayawardene et al. (2020). Crous et al. (2020b) introduced Trichomerium syzygii Crous, a hyphomycetous taxon. Hence, Trichomerium is reported as a holomorphic genus.

\section{Trichometasphaeria Munk} morph.

Kirk et al. (2008) reported that this genus has an ascochyta-like, coelomycetous asexual

\section{Trichophaea Boud.}

Healy et al. (2016) adopted Trichophaea, the older, sexually typified name over Dichobotrys Hennebert, the younger, asexually typified name.

Trichosphaerella E. Bommer et al.

Samuels \& Barr (1997) reported an acremonium-like asexual morph from this genus.

Triplosphaeria Kaz. Tanaka \& K. Hiray.

Tanaka et al. (2009) introduced this genus with tetrapoa-like asexual morphs.

Triseptata Boonmee \& Phook.

Boonmee et al. (2020) introduced this genus with a hyphomycetous asexual morph.

Tristratiperidium Daranag. et al.

Daranagama et al. (2016) introduced this genus with a hyphomycetous asexual morph.

\section{Trypetheliopsis Asahina}

Hongsanan et al. (2020b) reported conidiomata from this genus. 
Tubakia B. Sutton

This genus was reported with diplacella-like sexual morphs (Sogonov et al. 2008, Senanayake et al. 2017a, Braun et al. 2018).

Tubeufia Penz. \& Sacc.

Boonmee et al. (2014) provided the description for holomorph of this genus.

Tylophoron Nyl. ex Stizenb.

Tibell (1991) reported an asexual morph of Tylophoron moderatum.

\section{Tympanis Tode}

Johnston et al. (2014) proposed to adopt the older, sexually typified name Tympanis over the younger, asexually typified name, Sirodothis Clem. 1909 (= Pleurophomella Höhn. 1914).

Tyrannicordyceps Kepler \& Spatafora

Kepler et al. (2012) introduced this genus with a verticillium-like asexual morph.

\section{Uleoporthe Petr.}

Senanayake et al. (2017b) reported a coelomycetous asexual morph from U. orbiculata (Syd. $\&$ P. Syd.) Petr., the type species of this genus based on Cannon (2001).

\section{Uleothyrium Petr.}

Hongsanan et al. (2014) treated Septothyrella Höhn., an asexually typified name as a synonym of this genus.

\section{Unguiculariopsis Rehm 1909}

Johnston et al. (2014) proposed to adopt the older, sexually typified name, Unguiculariopsis over Deltosperma W.Y. Zhuang 1988.

Uzbekistanica Wanas. et al.

Wanasinghe et al. (2018) introduced this holomorphic genus, which has a coelomycetous asexual morph.

Vacuiphoma Valenz.-Lopez et al.

Valenzuela-Lopez et al. (2017) introduced this genus to accommodate Phoma bulgarica Aveskamp et al., which was phylogenetically distinct from Phoma sensu stricto in their phylogenetic analyses.

Vagicola K.W.T. Chethana \& K.D. Hyde

Jayasiri et al. (2015) reported an asexual morph from $V$. chlamydospora Jayasiri et al.

\section{Valdensia Peyronel}

Johnston et al. (2014) proposed to adopt Valdensia, the older, sexually typified name over Valdensinia Peyronel 1953 (= Asterobolus Redhead \& P.W. Perrin 1972), the younger, asexually typified name.

Valetoniellopsis Samuels \& M.E. Barr

Samuels \& Barr (1997) reported an acremonium-like asexual morph from this genus.

Valsalnicola D.M. Walker \& Rossman

Crous et al. (2012a) introduced this genus with a coelomycetous asexual morph. 
Valsaria Ces. \& De Not.

Jaklitsch et al. (2015) reported coelomycetous asexual morph from natural substrates. At the same time, Jaklitsch et al. (2015) reported hyphomycetous or coelomycetous asexual morphs from cultures.

Varicosporella Lechat \& J. Fourn.

Lechat \& Fournier (2015) introduced this genus with a fusarium-like asexual morph.

\section{Verkleyomyces Y. Marín \& Crous}

This genus was introduced with a coelomycetous asexual morph and confirmed its placement in Dermateaceae (Marin-Felix et al. 2017).

\section{Vibrissea Fr.}

Johnston et al. (2014) proposed to adopt Vibrissea, the older sexually typified name over Anavirga B. Sutton 1975, the younger, asexually typified name.

\section{Virgaria Nees}

Réblová et al. (2016a) proposed to adopt Virgaria, the older, asexually typified name rather than Ascovirgaria J.D. Rogers \& Y.-M. Ju 2002, the younger, sexually typified generic name.

Viridothelium Lücking et al. morph.

Hongsanan et al. (2020b) reported that this genus occasionally shows a pycnidial asexual

\section{Vizella Sacc.}

Hongsanan et al. (2020b) reported coelomycetous asexual morph of Vizella oleariae based on the holotype (Swart 1971).

\section{Volutella Fr.}

Luo \& Zhuang (2012) introduced Volutellonectria J. Luo \& W.Y. Zhuang with Volutella asexual morphs. Lombard et al. (2015) proposed to adopt Volutella over Volutellonectria.

\section{Wallrothiella Sacc.}

Crous et al. (2017) introduced Wallrothiella gmelinae Crous \& M.J. Wingf., which represents an asexual taxon.

\section{Westerdykella Stolk}

Crous et al. (2017) introduced Westerdykella centenaria Crous, a hyphomycetous taxon which resided in Westerdykella sensu stricto.

\section{Wuestneia Auersw. ex Fuckel}

Hyde et al. (2020b) accepted this genus with 'cylindrical asci with aseptate ascospores and ampuliform conidiogenous cells without conidiophores'.

Xanthonectria J. Fourn. \& P.-A. Moreau

Lechat et al. (2016) introduced this genus with an acremonium-like asexual morph.

\section{Xenocylindrocladium Decock et al.}

Schoch et al. (2000) introduced the genus Xenocalonectria Crous \& C.L. Schoch 2000 as the sexual morph of Xenocylindrocladium. Lombard et al. (2015) proposed to adopt the older, asexually typified name, Xenocylindrocladium over the younger, sexually typified name, Xenocalonectria. 
Xenodidymella Q. Chen \& L. Cai

This genus was introduced with a coelomycetous asexual morph.

Xenodium Syd.

Rossman et al. (2016) proposed to adopt the sexually typified name Xenodium, rather than Xenodiella Syd. 1935. Both names have been published in the same publication.

\section{Xerombrophila Baral}

This genus was introduced by Baral et al. (2013) with a hyphomycetous asexual morph.

Xeromyces Fraser et al.

Pitt et al. (2013) mentioned that this genus produces Frasierella asexual morph.

Xylaria Hill ex Schrank

Réblová et al. (2016a) proposed to adopt Xylaria, the older, sexually typified generic name, rather than Moelleroclavus Henn. 1902, Xylocoremium J.D. Rogers 1984, Arthroxylaria Seifert \& W. gams 2002 or Geniculisynnema Okane \& Nakagiri 2007.

Xylochrysis Réblová et al.

Réblová et al. (2014) introduced this genus with a hyphomycetous asexual morph.

Xylomelasma Réblová

Réblová (2006) introduced this genus and reported a hyphomycetous asexual morph.

Yunnanensis Karun. et al.

Karunarathna et al. (2017) introduced this holomorphic genus with a coelomycetous asexual morph and confirmed its placement in Phaeosphaeriaceae.

Yunnania H.Z. Kong

Woudenberg et al. (2017) showed that Fuscoannellis Sandoval-Denis et al. 2016 resides in Yunnania sensu stricto thus proposed to synonymize former generic name under latter generic name.

Zeloasperisporium R.F. Castañeda

Hongsanan et al. (2015b, 2020b) reported both sexual and asexual morph of this genus.

Zopfia Rabenh. (2012).

This genus was reported with a cladosporium-like asexual morph by Wijayawardene et al.

\section{Acknowledgements}

This work was supported by the National Natural Science Foundation of China (No. NSFC 31950410558, NSFC 31760013), Department of Science and Technology of Yunnan Province (No. 2018FB050), the State Key Laboratory of Functions and Applications of Medicinal Plants, Guizhou Medicial University (No. FAMP201906K); Science and Technology Department of Guizhou Province (QKHRCPT[2017]5101) and High-Level Talent Recruitment Plan of Yunnan Provinces ("Young Talents" Program and "High-End Foreign Experts" Program). Nalin N. Wijayawardene thanks Institute for Research and Development in Health and Social Care for a Honorary Research Associate position during the tenure of which this paper was finalised. 


\section{References}

Aa HA. van der. 1973 - Studies in Phyllosticta I. Studies in Mycology 5, 1-110.

Abeln ECA, de Pagter MA, Verkley GJM. 2000 - Phylogeny of Pezicula, Dermea and Neofabraea inferred from partial sequences of the nuclear ribosomal RNA gene cluster. Mycologia 92, 685-693.

Ainsworth GC. 1966 - A general purpose classification of the fungi. Bibliography of Systematic Mycology 4, 1-4.

Alvarez LV, Groenewald JZ, Crous PW. 2016 - Revising the Schizoparmaceae: Coniella and its synonyms Pilidiella and Schizoparme. Studies in Mycology 85, 1-34.

Ariyawansa HA, Hyde KD, Jayasiri SC, Buyck B et al. 2015 - Fungal diversity notes 111-252 taxonomic and phylogenetic contributions to fungal taxa. Fungal Diversity 75, 1-248.

Ariyawansa HA, Jones EBG, Suetrong S, Hyde KD. 2013. - Halojulellaceae, a new family of the order Pleosporales. Phytotaxa 130, 14-24.

Ariyawansa HA, Tanaka K, Thambugala KM, Phookamsak R et al. 2014 - A molecular phylogenetic reappraisal of the Didymosphaeriaceae (= Montagnulaceae). Fungal Diversity 68, 69-104.

Arx JA. 1973 - Centraalbureau voor Schimmelcultures, Progress Report 1972. Verhandelingen Koninklijke Nederlandse Akademie van Wetenschappen Afdeling Natuurkunde 61, 59-81.

Aveskamp MM, de Gruyter J, Woudenberg JHC et al. 2010 - Highlights of the Didymellaceae: a polyphasic approach to characterise Phoma and related pleosporalean genera. Studies in Mycology 65, 1-60.

Bao DF, Mckenzie EHC, Bhat DJ, Hyde KD et al. 2020 - Acrogenospora (Acrogenosporaceae, Minutisphaerales) Appears to be a very diverse genus. Frontiers in Microbiology 11, 1606,

Baral HO, Marson G, Bogale M, Untereiner WA. 2013 - Xerombrophila crystallifera, a new genus and species in the Helotiales. Mycological Progress 12, 475-488.

Baral HO, Weber E, Gams W, Hagedorn G et al. 2017 - Generic names in the Orbiliaceae (Orbiliomycetes) and recommendations on which names should be protected or suppressed. Mycological Progress 17, 5-31.

Bensch K, Braun U, Groenewald JZ, Crous PW. 2012 - The genus Cladosporium. Studies in mycology 72, 1-401.

Berthet P. 1964 - Formes conidiennes de divers discomycetes. Bulletin de la Société mycologique de France 80, 125-149.

Beyma FHV. 1940 - Beschreibung einiger neuer Pilzarten aus dem Centraalbureau voor Schimmelcultures, Baarn (Nederland), VI. Mitteilung. Antonie van Leeuwenhoek 6, 263 290.

Boehm EW, Mugambi GK, Miller AN, Huhndorf SM et al. 2009 - A molecular phylogenetic reappraisal of the Hysteriaceae, Mytilinidiaceae and Gloniaceae (Pleosporomycetidae, Dothideomycetes) with keys to world species. Studies in Mycology 64, 49-83.

Boonmee S, Calabon MS, Phookamsak R, Elgorban AM. 2020 - Triseptata sexualis gen. et sp. nov. in Latoruaceae (Pleosporales). Phytotaxa 447, 252-64.

Boonmee S, Phookamsak R, Hongsanan S, Doilom M et al. 2017 - Mycosphere notes 51-101. Revision of genera in Perisporiopsidaceae and Pseudoperisporiaceae and other Ascomycota genera incertae sedis. Mycosphere 8, 1695-1801.

Boonmee S, Rossman AY, Liu JK, Li WJ et al. 2014 - Tubeufiales, ord. nov., integrating sexual and asexual generic names. Fungal Diversity 68, 239-298.

Boonmee S, Zhang Y, Chomnunti P, Chukeatirote E et al. 2011 - Revision of lignicolous Tubeufiaceae based on morphological reexamination and phylogenetic analysis. Fungal Diversity 51, 63-102.

Brahmanage RS, Lu YZ, Bhat DJ, Wanasinghe DN et al. 2017 - Phylogenetic investigations on freshwater fungi in Tubeufiaceae (Tubeufiales) reveals the new genus Dictyospora and new species Chlamydotubeufia aquatica and Helicosporium flavum. Mycosphere 8, 917-933. 
Braun U, Crous PW, Dugan FM, Groenewald JZ, de Hoog GS. 2003 - Phylogeny and taxonomy of cladosporium-like hyphomycetes, including Davidiella gen. nov., the teleomorph of Cladosporium s. str. Mycological Progress 2, 3-18.

Braun U, Nakashima C, Crous PW, Groenewald JZ et al. 2018 - Phylogeny and taxonomy of the genus Tubakia s. lat. Fungal Systematics and Evolution 1, 41-99.

Braun U. 2013 - Proposals to conserve the teleomorph-typified name Blumeria against the anamorph-typified name Oidium and twenty-two teleomorph-typified powdery mildew species names against competing anamorph-typified names (Ascomycota: Erysiphaceae). Taxon 62, 1328-1331.

Butin H, Holdenrieder O, Sieber TN. 2013 - The complete life cycle of Petrakia echinata. Mycological Progress 12, 427-435.

Câmara MP, Ramaley AW, Castlebury LA, Palm ME. 2003 - Neophaeosphaeria and Phaeosphaeriopsis segregates of Paraphaeosphaeria. Mycological research 107, 516-522.

Candoussau F, Boqueras M, Gómez-Bolea A, Laessoe T, et al. 2007 - Observations on Neobarya, including new species and new combinations. Sydowia 59, 179-215.

Cannon PF, Alcorn JL. 1994 - Omnidemptus affinis gen. et sp. nov., teleomorph of Mycoleptodiscus affinis sp. nov. Mycotaxon 51, 483-487.

Cannon PF. 2001 - Rediscovery and redescription of the genus Uleoporthe (Melanconidaceae). Fungal Diversity 7, 17-25.

Carmichael JW, Kendrick BW, Conners IL, Sigler L. 1980 - Genera of Hyphomycetes. University of Alberta Press, Edmonton.

Chaverri P, Bischoff JF, Evans HC, Hodge KT. 2005 - Regiocrella, a new entomopathogenic genus with a pycnidial anamorph and its phylogenetic placement in the Clavicipitaceae. Mycologia 97, 1225-1237.

Chaverri P, Liu M, Hodge KT. 2008 - A monograph of the entomopathogenic genera Hypocrella, Moelleriella, and Samuelsia gen. nov. (Ascomycota, Hypocreales, Clavicipitaceae), and their aschersonia-like anamorphs in the Neotropics. Studies in Mycology 60, 1-66.

Chaverri P, Salgado C, Hirooka Y, Rossman AY et al. 2011 - Delimitation of Neonectria and Cylindrocarpon (Nectriaceae, Hypocreales, Ascomycota) and related genera with Cylindrocarpon-like anamorphs. Studies in Mycology 68, 57-78.

Cheewangkoon R, Groenewald JZ, Verkley GJ, Hyde KD et al. 2010 - Re-evaluation of Cryptosporiopsis eucalypti and Cryptosporiopsis-like species occurring on Eucalyptus leaves. Fungal Diversity 44, 89-105.

Chen Q, Jiang JR, Zhang GZ, Cai L, Crous PW. 2015 - Resolving the Phoma enigma. Studies in Mycology 82, 137-217.

Chethana T, Liu M, Ariyawansa HA, Konta S et al. 2015 - Splanchnonema-like species in Pleosporales: introducing Pseudosplanchnonema gen. nov. in Massarinaceae. Phytotaxa 231, 133-144.

Chomnunti P, Schoch CL, Aguirre-Hudson B, Ko-Ko TW et al. 2011 - Capnodiaceae. Fungal Diversity $51,103-134$.

Ciferrit R. 1957 - Sulla forma picnidica della Stictis panizzei De Not. Atti dell'Istituto Botanico della Università e Laboratorio Crittogamico di Pavia. 14, 271-277.

Constantinescu O, Holm K, Holm L. 1989 - Teleomorph-anamorph connections in Ascomycetes. 1-3. Stanhughesia (Hyphomycetes) new genus, the anamorph of Ceramothyrium. Studies in Mycology 31, 69-84.

Crous PW, Braun U, Groenewald JZ. 2007a - Mycosphaerella is polyphyletic. Studies in Mycology 58, 1-32.

Crous PW, Braun U. 2003 - Mycosphaerella and its anamorphs: 1. Names published in Cercospora and Passalora. CBS Biodiversity Series No. 1. Westerdijk Fungal Biodiversity Institute, Utrecht, the Netherlands.

Crous PW, Carris LM, Giraldo A, Groenewald JZ et al. 2015a - The Genera of Fungi - fixing the application of the type species of generic names - G 2: Allantophomopsis, Latorua, 
Macrodiplodiopsis, Macrohilum, Milospium, Protostegia, Pyricularia, Robillarda, Rotula, Septoriella, Torula, and Wojnowicia. IMA Fungus 6, 163-198.

Crous PW, Groenewald JZ. 2017 - The Genera of Fungi - G 4: Camarosporium and Dothiora. IMA Fungus 8, 131-152.

Crous PW, Groenewald JZ, Gams W. 2003 - Eyespot of cereals revisited: ITS phylogeny reveals new species relationships. European Journal of Plant Pathology 109, 841-850.

Crous PW, Mohammed C, Glen M, Verkley GJ, Groenewald JZ. 2007b - Eucalyptus microfungi known from culture. 3. Eucasphaeria and Sympoventuria genera nova, and new species of Furcaspora, Harknessia, Heteroconium and Phacidiella. Fungal Diversity 25, 19-36.

Crous PW, Müller M, Sánchez RM, Giordano L et al. 2015b - Resolving Tiarosporella spp. allied to Botryosphaeriaceae and Phacidiaceae. Phytotaxa 202, 73-93.

Crous PW, Schumacher RK, Akulov A, Thangavel R et al. 2019 - New and interesting fungi. 2. Fungal Systematics and Evolution 3, 57-134.

Crous PW, Schumacher RK, Wingfield MJ, Lombard L et al. 2015c - Fungal systematics and evolution: FUSE 1. Sydowia 67, 81-118.

Crous PW, Schumacher RK, Wood AR, Groenewald JZ. 2020a - The Genera of Fungi-G5: Arthrinium, Ceratosphaeria, Dimerosporiopsis, Hormodochis, Lecanostictopsis, Lembosina, Neomelanconium, Phragmotrichum, Pseudomelanconium, Rutola, and Trullula. Fungal Systematics and Evolution 5, 77-98.

Crous PW, Shivas RG, Quaedvlieg W, van der Bank M et al. 2014a - Fungal Planet Description Sheets: 214-280. Persoonia 32, 184-306.

Crous PW, Shivas RG, Wingfield MJ, Summerell BA. 2012a - Fungal Planet description sheets: 128-153. Persoonia: Molecular Phylogeny and Evolution of Fungi 29, 146-201.

Crous PW, Slippers B, Wingfeld MJ, Rheeder J et al. 2006 - Phylogenetic lineages in the Botryosphaeriaceae. Studies in Mycology 55, 235-253.

Crous PW, Summerell BA, Carnegie AJ, Wingfield MJ et al. 2009 - Unravelling Mycosphaerella: do you believe in genera? Persoonia 23, 99-118.

Crous PW, Summerell BA, Shivas RG et al. 2012b - A re-appraisal of Harknessia (Diaporthales), and the introduction of Harknessiaceae fam. nov. Persoonia 28, 49-65.

Crous PW, Wingfield MJ, Burgess TI, Hardy GS et al. 2016b - Fungal Planet description sheets: 469-557. Persoonia: Molecular Phylogeny and Evolution of Fungi 37, 218-403.

Crous PW, Wingfield MJ, Burgess TI, Hardy GS et al. 2018 - Fungal Planet description sheets: 716-784. Persoonia 40, 240-393.

Crous PW, Wingfield MJ, Burgess TI, Hardy GS. 2017 - Fungal Planet description sheets: 558624. Persoonia 38, 240-384.

Crous PW, Wingfield MJ, Guarro J, Cheewangkoon R et al. 2013 - Fungal Planet description sheets: 154-213. Persoonia 31,188-296.

Crous PW, Wingfield MJ, Guarro J, Hernández-Restrepo M et al. 2015d - Fungal Planet Description Sheets: 320-370. Persoonia 34, 167-266.

Crous PW, Wingfield MJ, Richardson DM, Le Roux JJ et al. 2016a - Fungal Planet description sheets: 400-468. Persoonia 36, 316-458.

Crous PW, Wingfield MJ, Schumacher RK, Akulov A et al. 2020b - New and Interesting Fungi. 3. Fungal Systematics and Evolution 6, 157-231.

Crous PW, Wingfield MJ, Schumacher RK, Summerell BA et al. 2014b - Fungal Planet Description Sheets: 281-319. Persoonia 33, 212-289.

Dai DQ, Bhat DJ, Liu JK, Chukeatirote E et al. 2012 - Bambusicola, a new genus from bamboo with asexual and sexual morphs. Cryptogamie Mycologie 33, 363-379.

Dai DQ, Phookamsak R, Wijayawardene NN, Li WJ et al. 2017 - Bambusicolous fungi. Fungal Diversity 82, 1-105.

Dai DQ, Wijayawardene NN, Tang LZ, Liu C et al. 2019 - Rubroshiraia gen. nov. a second hypocrellin-producing genus in Shiraiaceae (Pleosporales). MycoKeys 58, 1-26. 
Damm U, Crous PW, Fourie PH. 2008 - A fissitunicate ascusmechanism in the alosphaeriaceae, and novel species of Jattaea and Calosphaeria on Prunus wood. Persoonia 20, 39-52.

Daranagama DA, Camporesi E, Liu XZ, Bhat DJ et al. 2016 - Tristratiperidium microsporum gen. et sp. nov. (Xylariales) on dead leaves of Arundo plinii. Mycological progress 15, 1-8.

Daranagama DA, Camporesi E, Tian Q, Liu X et al. 2015 - Anthostomella is polyphyletic comprising several genera in Xylariaceae. Fungal Diversity 73, 203-238.

Darker GD. 1963 A new genus of Phacidiaceae on Picea mariana. Canadian Journal of Botany 41, 1389-1393.

Day MJ, Hall JC, Currah RS. 2012 - Phialide arrangement and character evolution in the helotialean anamorph genera Cadophora and Phialocephala. Mycologia 104, 371-381.

Dayarathne MC, Maharachchikumbura SS, Jones EB, Goonasekara ID et al. 2017 Neophyllachora gen. nov. (Phyllachorales), three new species of Phyllachora from Poaceae and resurrection of Polystigmataceae (Xylariales). Mycosphere 8, 1598-1625

Dayarathne MC, Wanasinghe DN, Devadatha B, Abeywickrama P et al. 2020 - Modern taxonomic approaches to identifying diatrypaceous fungi from marine habitats, with a novel genus Halocryptovalsa Dayarathne \& KD Hyde, gen. nov. Cryptogamie, Mycologie 41, 21-67.

De Beer ZW, Duong TA, Barnes I, Wingfield BD et al. 2014 - Redefining Ceratocystis and allied genera. Studies in Mycology 79, 187-219.

De Beer ZW, Duong TA, Wingfield MJ. 2016 - The divorce of Sporothrix and Ophiostoma: solution to a problematic relationship. Studies in Mycology 83, 165-91.

De Beer ZW, Marincowitz S, Duong TA, Wingfield MJ. 2017 - Bretziella, a new genus to accommodate the oak wilt fungus, Ceratocystis fagacearum (Microascales, Ascomycota). MycoKeys 27, 1-19.

De Jong SN, Levesque CA, Verkley GJM, Abeln ECA et al. 2001 - Phylogenetic relationships among Neofabraea species causing tree cankers and bull's-eye rot of apple based on DNA sequencing of ITS nuclear rDNA, mitochondrial rDNA, and the beta-tubulin gene. Mycological Research 105, 658-669.

Döbbeler P, Davison PG. 2018 - Frullania as a hotspot for hypocrealean ascomycetes: ten new species from Southeastern North America. Nova Hedwigia 106, 1. DOI: 10.1127/nova_hedwigia/2017/0428

Doilom M, Dissanayake AJ, Wanasinghe DN, Boonmee S et al. 2017 - Microfungi on Tectona grandis (teak) in Northern Thailand. Fungal Diversity 82, 107-182.

Dong W, Wang B, Hyde KD et al. 2020 - Freshwater Dothideomycetes. Fungal Diversity 105, 319-575.

Doveri F, Pecchia S, Vergara M, Sarrocco S, Vannacci G. 2011 - A comparative study of Neogymnomyces virgineus, a new keratinolytic species from dung, and its relationships with the Onygenales. Fungal Diversity 52, 13-34.

Ekanayaka AH, Hyde KD, Gentekaki E, McKenzie EHC et al. 2019 - Preliminary classification of Leotiomycetes. Mycosphere 10, 310-489.

Ekanayaka AH, Hyde KD, Jones EBG, Zhao Q. 2018 - Orbiliaceae from Thailand. Mycosphere 9, $155-168$.

Ellis MB 1971 - Dematiaceous Hyphomycetes. Commonwealth Mycological Institute, Kew, England.

Ellis MB. 1976 - More Dematiaceous Hyphomycetes. Commonwealth Mycological Institute, Kew

Eriksson O. 1982 - Notes on ascomycetes and coelomycetes from NW Europe. Mycotaxon 15, 189-202.

Ertz D, Diederich P, Lawrey JD, Berger F et al. 2015 - Phylogenetic insights resolve Dacampiaceae (Pleosporales) as polyphyletic: Didymocyrtis (Pleosporales, Phaeosphaeriaceae) with Phoma-like anamorphs resurrected and segregated from Polycoccum (Trypetheliales, Polycoccaceae fam. nov.). Fungal Diversity 74, 53-89. 
Ertz D, Lawrey JD, Common RS, Diederich P. 2014 - Molecular data resolve a new order of Arthoniomycetes sister to the primarily lichenized Arthoniales and composed of black yeasts, lichenicolous and rock-inhabiting species. Fungal Diversity 66, 113-137.

Fan XL, Bezerra JDP, Tian CM, Crous PW. 2018 - Families and genera of diaporthalean fungi associated with canker and dieback of tree hosts. Persoonia 40, 119-134.

Fehrer J, Réblová M, Bambasová V, Vohník M. 2019 - The root-symbiotic Rhizoscyphus ericae aggregate and Hyaloscypha (Leotiomycetes) are congeneric: Phylogenetic and experimental evidence. Studies in Mycology 92,195-225.

Fernández FA, Miller AN, Huhndorf SM, Huhndorf SM et al. 2006 - Systematics of the genus Chaetosphaeria and its allied genera: morphological and phylogenetic diversity in north temperate and neotropical taxa. Mycologia 98, 121-130.

Fournier J, Lechat C. 2010 - Phomatospora luteotingens sp. nov., a new aquatic species of Phomatospora from France and Spain. Mycosphere 1, $39-43$.

Fournier J, Stadler M, Hyde KD, Duong ML. 2010 - The new genus Rostrohypoxylon and two new Annulohypoxylon species from Northern Thailand. Fungal Diversity 40, 23-36.

Fu CH, Hsieh HM, Chen CY, Chang TT et al. 2013 - Ophiodiaporthe cyatheae gen. et sp. nov., a diaporthalean pathogen causing a devastating wilt disease of Cyathea lepifera in Taiwan. Mycologia 105, 861-72.

Gams W, Stielow B, Gräfenhan T, Schroers HJ. 2019 - The ascomycete genus Niesslia and associated monocillium-like anamorphs. Mycological Progress18, 5-76.

Ginns J. 2011 - Leucogyrophana (Aphyllophorales): identification of species. Canadian Journal of Botany 56, 1953-1973.

Giraldo A, Crous PW, Schumacher RK, Cheewangkoon R et al. 2017 - The Genera of Fungi - G3: Aleurocystis, Blastacervulus, Clypeophysalospora, Licrostroma, Neohendersonia and Spumatoria. Mycological Progress 16, 325-48.

González CD, Chaverri P. 2017 - Corinectria, a new genus to accommodate Neonectria fuckeliana and C. constricta sp. nov. from Pinus radiata in Chile. Mycological Progress 16, 1015-27.

Groves JW. 1936 - Ascocalyx abietis and Bothrodiscus pinicola. Mycologia 28, 451-462.

Grum-Grzhimaylo AA, Debets AJ, van Diepeningen AD, Georgieva ML et al. 2013 -Sodiomyces alkalinus, a new holomorphic alkaliphilic ascomycete within the Plectosphaerellaceae. Persoonia 31, 147-158.

Grunig CR, Queloz V, Duo A, Sieber TN. (2009) Phylogeny of Phaeomollisia piceae gen. sp. nov.: a dark, septate, coniferneedle endophyte and its relationships to Phialocephala and Acephala. Mycological Research 113, 207-221.

Gruyter J de, Woudenberg JHC, Aveskamp MM, Verkley GJM et al. 2012 - Redisposition of Phoma-like anamorphs in Pleosporales. Studies in Mycology 75, 1-36.

Gryzenhout M, Myburg H, Hodges CS, Wingfield BD, Wingfield MJ. 2006 - Microthia, Holocryphia and Ursicollum, three new genera on Eucalyptus and Coccoloba for fungi previously known as Cryphonectria. Studies in Mycology 55, 35-52.

Guterres DC, Galvão-Elias S, de Souza BC, Pinho DB et al. 2018 - Taxonomy, phylogeny, and divergence time estimation for Apiosphaeria guaranitica, a Neotropical parasite on bignoniaceous hosts. Mycologia 110, 526-45.

Hashimoto A, Hirayama K, Takahashi H, Matsumura M et al. 2018 - Resolving the Lophiostoma bipolare complex: insights into complicated delimitations within the Lophiostomataceae genera. Studies in Mycology 90, 161-189.

Hashimoto A, Matsumura M, Hirayama K, Tanaka K. 2017 - Revision of Lophiotremataceae (Pleosporales, Dothideomycetes): Aquasubmersaceae, Cryptocoryneaceae, and Hermatomycetaceae fam. nov. Persoonia 39, 51-73.

Hassan O, Chang T. 2019 - Phylogenetic and morphological reassessment of Mycosphaerella nawae, the causal agent of circular leaf spot in persimmon. Plant Disease 103, 200-213.

Healy R, Pfister DH, Rossman AY, Ludmila M et al. 2016 - Competing sexual-asexual generic names of Pezizomycetes and recommendations for use. IMA Fungus 7, 285-288. 
Heluta V, Takamatsu S, Harada M, Voytyuk S. 2010 - Molecular phylogeny and taxonomy of Eurasian Neoerysiphe species infecting Asteraceae and Geranium. Persoonia 24, 81-92.

Hernández-Restrepo M, Groenewald JZ, Crous PW. 2016 - Taxonomic and phylogenetic reevaluation of Microdochium, Monographella and Idriella. Persoonia 36, 57-82.

Herrera CS, Rossman AY, Samuels GJ, Lechat C, Chaverri P. 2013 - Revision of the genus Corallomycetella with Corallonectria gen. nov. for C. jatrophae (Nectriaceae, Hypocreales). Mycosystema 32, 518-44.

Hesseltine CW. 1953 - Study of trickling filter fungi. Bulletin of the Torrey Botanical Club 80, 507-514.

Hirooka Y, Tanney JB, Nguyen HD, Seifert KA. 2016 - Xerotolerant fungi in house dust: taxonomy of Spiromastix, Pseudospiromastix and Sigleria gen. nov. in Spiromastigaceae (Onygenales, Eurotiomycetes). Mycologia 108, 135-56.

Hodge KT. 2003 - Clavicipitaceous anamorphs. Clavicipitalean Fungi. CRC Press.

Holm K, Holm K, Barr Me. 1999 - Once again Discosphaerina. Karstenia 39, 59-63.

Hongsanan S, Hyde KD, Phookamsak R, Wanasinghe DN et al. 2020a - Refined families of Dothideomycetes: Dothideomycetidae and Pleosporomycetidae. Mycosphere 11, 1553-2107.

Hongsanan S, Hyde KD, Phookamsak R, Wanasinghe DN et al. 2020b - Refined families of Dothideomycetes: Orders and families incertae sedis. Fungal Diversity 105, 17-318.

Hongsanan S, Li YM, Liu JK, Hofmann T et al. 2014 - Revision of genera in Asterinales. Fungal Diversity 68, 1-68.

Hongsanan S, Tian Q, Bahkali AH, Yang JB et al. 2015b - Zeloasperisporiales ord. nov., and two new species of Zeloasperisporium. Cryptogamie, Mycologie 36, 301-17.

Hongsanan S, Tian Q, Peršoh D, Zeng XY et al. 2015a - Meliolales. Fungal Diversity 74, 91-141.

Hosagoudar VB, Riju MC. 2013 - Foliicolous fungi of Silent Valley National Park, Kerala, India. Journal of Threatened Taxa 5, 3701-3788.

Hosoya T, Otani Y. 1995 - Gelatinipulvinella astraeicola gen. et sp. nov., a fungicolous discomycete and its anamorph. Mycologia 87, 689-696.

Hou LW, Groenewald JZ, Pfenning LH, Yarden O et al. 2020 - The phoma-like dilemma. Studies in Mycology 96, 309-396.

Houbraken J, Kocsubé S, Visagie CM, Yilmaz N et al. 2020 - Classification of Aspergillus, Penicillium, Talaromyces and related genera (Eurotiales): an overview of families, genera, subgenera, sections, series and species. Studies in Mycology 95, 5-169.

Houbraken JA, Samson RA. 2011 - Phylogeny of Penicillium and the segregation of Trichocomaceae into three families. Studies in Mycology 70, 1-51.

Hughes SJ, Pirozynski KA. 1994 - New Zealand fungi 34. Endomeliola dingleyae, a new genus and new species of Meliolaceae. New Zealand Journal of Botany 32, 53-9.

Huhndorf SM, Miller AN, Greif M, Samuels GJ. 2009 - Amplistroma gen. nov. and its relation to Wallrothiella, two genera with globose ascospores and acrodontium-like anamorphs. Mycologia 101, 904-19.

Huhndorf SM. 1992 - Neotropical Ascomycetes 2. Hypsostroma, a new genus from the Dominican Republic and Venezuela. Mycologia 84, 750-8.

Hyde KD, Chaiwan N, Norphanphoun C, Boonmee S et al. 2018 - Mycosphere notes 169-224. Mycosphere 9, 271-430.

Hyde KD, Dong Y, Phookamsak R, Jeewon R et al. 2020a - Fungal diversity notes 1151-1276: taxonomic and phylogenetic contributions on genera and species of fungal taxa. Fungal Diversity 100, 5-277.

Hyde KD, Hongsanan S, Jeewon R, Bhat DJ et al. 2016 - Fungal diversity notes 367-491: taxonomic and phylogenetic contributions to fungal taxa. Fungal Diversity 80, 1-270.

Hyde KD, Jones EBG, Lui J-K, Ariyawansa H et al. 2013 - Families of Dothideomycetes. Fungal Diversity 63, 1-313.

Hyde KD, Norphanphoun C, Abreu VP, Bazzicalupo A et al. 2017 - Fungal diversity notes 603708: taxonomic and phylogenetic notes on genera and species. Fungal Diversity 87, 1-235. 
Hyde KD, Norphanphoun C, Maharachchikumbura SS, Bhat DJ et al. 2020b - Refined families of Sordariomycetes. Mycosphere 11, 305-1059.

Hyde KD, Tennakoon DS, Jeewon R, Bhat DJ et al. 2019 - Fungal diversity notes 1036-1150: taxonomic and phylogenetic contributions on genera and species of fungal taxa. Fungal Diversity 96, 1-242.

Index Fungorum (2021) http://www.indexfungorum.org/Names/Names.asp (Accessed in January 2021).

Jaklitsch WM, Checa J, Blanco MN, Olariaga I et al. 2018 - A preliminary account of the Cucurbitariaceae. Studies in Mycology 90, 71-118.

Jaklitsch WM, Fournier J, Dai DQ, Hyde KD. Voglmayr H. 2015 - Valsaria and the Valsariales. Fungal Diversity 73, 159-202.

Jaklitsch WM, Olariaga I, Voglmayr H. 2016 - Teichospora and the Teichosporaceae. Mycological Progress 15, 31.

Jaklitsch WM, Samuels KPJ. 2008 - Reconsideration of (Hypocreales, Hypocreaceae). Mycologia, 100, 962-984.

Jaklitsch WM, Voglmayr H. 2016 - Hidden diversity in Thyridaria and a new circumscription of the Thyridariaceae. Studies in Mycology 85, 35-64.

Jaklitsch WM, Voglmayr H. 2017 - Three former taxa of Cucurbitaria and considerations on Petrakia in the Melanommataceae. Sydowia 69, 81-95.

Jaklitsch WM, Voglmayr H. 2019 - Fenestelloid clades of the Cucurbitariaceae. Persoonia 44, 140.

Jayasiri SC, Hyde KD, Jones EB, Peršoh D et al. 2018 - Taxonomic novelties of hysteriform Dothideomycetes. Mycosphere 9, 803-37.

Jayasiri SC, Hyde KD, Jones EBG, McKenzie EHC et al. 2019 - Diversity, morphology and molecular phylogeny of Dothideomycetes on decaying wild seed pods and fruits. Mycosphere $10,1-186$.

Jayasiri SC, Hyde KD, Jones EG, Ariyawansae HA et al. 2017 - A New Hysteriform Dothideomycete (Gloniaceae, Pleosporomycetidae Incertae sedis), Purpurepithecium murisporum gen. et sp. nov. on Pine Cone Scales. Cryptogamie Mycologie 38, 241-251.

Jayasiri SC, Wanasinghe DN, Ariyawansa HA, Jones EBG et al. 2015 - Two novel species of Vagicola (Phaeosphaeriaceae) from Italy. Mycosphere 6, 716-728.

Jiang N, Fan X, Tian C, Crous PW. 2020a - Reevaluating Cryphonectriaceae and allied families in Diaporthales. Mycologia 112, 267-92.

Jiang SH, Hawksworth DL, Lücking R, Wei JC. 2020c - A new genus and species of foliicolous lichen in a new family of Strigulales (Ascomycota: Dothideomycetes) reveals remarkable class-level homoplasy. IMA Fungus 11, 1.

Jiang SH, Lucking R, Xavier-Leite AB, Caceres ME et al. 2020b - Reallocation of foliicolous species of the genus Strigula into six genera (lichenized Ascomycota, Dothideomycetes, Strigulaceae). Fungal Diversity 102, 257-291.

Johnston PR. 1985 - Anamorphs of the ostropalean genera Schizoxylon and Acarosporina. Mycotaxon 24, 349-360.

Johnston PR. 1998 - The Bloxamia anamorph of Bisporella discedens. Mycotaxon 31, 345-350.

Johnston PR, Rogers JD, Park D, Martin NA. 2016 - Entalbostroma erumpens gen. et sp. nov. (Xylariaceae) from Phormium in New Zealand. Mycotaxon 131, 765-71.

Johnston PR, Seifert KA, Stone JK, Rossman AY, Marvanová L. 2014 - Recommendations on generic names competing for use in Leotiomycetes (Ascomycota). IMA Fungus 5, 91-120.

$\mathrm{Ju}$ YM, Rogers JD. 2001 - Xylaria cranioides and Poronia pileiformis and their anamorphs in culture, and implications for the status of Penzigia. Mycological Research 105(9), 11341146.

Ju YM, San Martin González F, Rogers JD. 1993 - Three xylariaceous fungi with scolecosporous conidia. Mycotaxon 47, 219-28. 
Karunarathna A, Papizadeh M, Senanayake IC, Jeewon R et al. 2017 - Novel fungal species of Phaeosphaeriaceae with an asexual/sexual morph connection. Mycosphere 8, 1818-1834.

Kepler RM, Humber RA, Bischoff JF, Rehner SA. 2014 - Clarification of generic and species boundaries for Metarhizium and related fungi through multigene phylogenetics. Mycologia $106,811-829$.

Kepler RM, Luangsa-Ard JJ, Hywel-Jones NL, Quandt CA et al. 2017 - A phylogenetically-based nomenclature for Cordycipitaceae (Hypocreales). IMA Fungus 8, 335-353.

Kepler RM, Sung GH, Harada Y, Tanaka K et al. 2012 - Host jumping onto close relatives and across kingdoms by Tyrannicordyceps (Clavicipitaceae) gen. nov. and Ustilaginoidea (Clavicipitaceae). American Journal of Botany 99, 552- 561.

Kirk PM, Cannon PF, Minter DW, Stalpers JA. 2008 - Ainsworth \& Bisby's dictionary of the fungi, 10th edn. CABI, Wallingford.

Kirk PM, Stalpers JA, Braun U, Crous PW et al. 2013 - A withoutprejudice list of generic names of fungi for protection under the International Code of Nomenclature for algae, fungi, and plants. IMA Fungus 4, 381-443.

Klaubauf S, Tharreau D, Fournier E, Groenewald JZ et al. 2014 - Resolving the polyphyletic nature of Pyricularia (Pyriculariaceae). Studies in Mycology 79, 85-120.

Kohlmeyer J, Volkmann-Kohlmeyer B, Eriksson OE. 1995 - Fungi on Juncus roemerianus 2. New dictyosporous Ascomycetes. Botanica Marina 38, 1651-1674.

Korf RP, Pearson RC, Zhuang WY, Dubos B. 1986 - Pseudopezicula (Helotiales, Peziculoideae), a new discomycete genus for pathogens causing an angular leaf scorch of grapes ("Rotbrenner"). Mycotaxon 26, 457-471.

Lechat C, Fournier J, Moreau PA. 2016 - Xanthonectria, a new genus for the nectrioid fungus Nectria pseudopeziza. Ascomycete. org. 8, 172-178.

Lechat C, Fournier J. 2015 - Varicosporella, a new aquatic genus in the Nectriaceae from France. Ascomycete.org 7, 1-8.

Lechat C, Fournier J. 2016 - Lasionectriella, a new genus in the Bionectriaceae, with two new species from France and Spain, L. herbicola and L. rubioi. Ascomycete.org 8, 62.

Lechat C, Fournier J. 2018 - Flammocladiella decora a new combination to accommodate the hypocrealean fungus Nectria decora. Ascomycete.org 10, 48-54.

Leonard KJ, Suggs EG. 1974 - Setosphaeria prolata, the ascigerous state of Exserohilum prolatum. Mycologia 66, 281-297.

Leuchtmann A, Müller E. 1986 - Über Thyridium vestitum und sein Anamorph (Ascomycetes). Botanica Helvetica 96, 283-287.

Li GJ, Hyde KD, Zhao RL, Hongsanan S. et al. 2016 - Fungal diversity notes 253-366: taxonomic and phylogenetic contributions to fungal taxa. Fungal Diversity 78, 1-237.

Li SP, Li P, Dong TTX, Tsim KWK. 2001 - Anti-oxidation activity of different types of natural Cordyceps sinensis and cultured Cordyceps mycelia. Phytomedicine 8, 207-212.

Li WJ, Bhat DJ, Camporesi E, Tian Q. et al. 2015 - New asexual morph taxa in Phaeosphaeriaceae. Mycosphere 6, 681-708.

Li WJ, McKenzie EH, Liu JK, Bhat DJ et al. 2020 - Taxonomy and phylogeny of hyaline-spored coelomycetes. Fungal Diversity 100, 279-801.

Li WL, Luo ZL, Liu JK, Bhat DJ et al. 2017 - Lignicolous freshwater fungi from China I: Aquadictyospora lignicola gen. et sp. nov. and new record of Pseudodictyosporium wauense from northwestern Yunnan Province. Mycosphere 8, 1587- 1597.

Liu F, Hu DM, Cai L. 2012a - Conlarium duplumascospora gen. et. sp. nov. and Jobellisia guangdongensis sp. nov. from freshwater habitats in China. Mycologia 104, 1178-1186.

Liu JK, Hyde KD, Jones EG, Ariyawansa HA et al. 2015 - Fungal diversity notes 1-110: taxonomic and phylogenetic contributions to fungal species. Fungal Diversity 72, 1-97.

Liu JK, Lu YZ, Cheewangkoon R, To-Anun C. 2018 - Phylogeny and morphology of Helicotubeufia gen. nov., with three new species in Tubeufiaceae from aquatic habitats. Mycosphere 9, 495-509. 
Liu JK, Phookamsak R, Dai DQ, Tanaka K et al. 2014 - Roussoellaceae, a new pleosporalean family to accommodate the genera Neoroussoella gen. nov., Roussoella and Roussoellopsis. Phytotaxa 181, 1-33.

Liu JK, Phookamsak R, Doilom M, Wikee S et al. 2012b - Towards a natural classification of Botryosphaeriales. Fungal Diversity 57, 149-210.

Lombard L, Crous PW, Wingfield BD, Wingfield MJ. 2010 - Phylogeny and systematics of the genus Calonectria. Studies in Mycology 66, 31-69.

Lombard L, Houbraken J, Decock C, Samson RA et al. 2016 - Generic hyper-diversity in Stachybotriaceae. Persoonia 36, 156-246.

Lombard L, Serrato-Diaz LM, Cheewangkoon R et al. 2014a - Phylogeny and taxonomy of the genus Gliocephalotrichum. Persoonia 32: 127-140.

Lombard L, Van Der Merwe A, Groenewald JZ, Crous PW. 2014b - Lineages in Nectriaceae: reevaluating the generic status of Ilyonectria and allied genera. Phytopathologia Mediterranea 515-32.

Lombard L, van der Merwe NA, Groenewald JZ, Crous PW. 2015 - Generic concepts in Nectriaceae. Studies in Mycology 80, 189-245.

Lu YZ, Boonmee S, Bhat DJ, Hyde KD et al. 2017 - Helicosporium luteosporum sp. nov. and Acanthohelicospora aurea (Tubeufiaceae, Tubeufiales) from terrestrial habitats. Phytotaxa 319, 241-253.

Lu YZ, Liu JK, Hyde KD, Jeewon R et al. 2018 - A taxonomic reassessment of Tubeufiales based on multi-locus phylogeny and morphology. Fungal Diversity 92, 131-344.

Luangsa-ard JJ, Mongkolsamrit S, Thanakitpipattana D, Khonsanit A et al. 2017 - Clavicipitaceous entomopathogens: New species in Metarhizium and a new genus Nigelia. Mycological Progress 16, 369-391.

Luo J, Zhuang WY. 2012 - Volutellonectria (Ascomycota, Fungi), a new genus with Volutella anamorphs. Phytotaxa 44, 1-10.

Maharachchikumbura SS, Ariyawansa HA, Wanasinghe DN, Dayarathne MC et al. 2019 Phylogenetic classification and generic delineation of Hydeomyces desertipleosporoides gen. et sp. nov., (Phaeosphaeriaceae) from Jebel Akhdar Mountain in Oman. Phytotaxa 391, 28-38.

Maharachchikumbura SSN, Hyde KD, Jones EBG, Mckenzie EHC et al. 2015 - Towards a natural classification and backbone tree for Sordariomycetes. Fungal Diversity, 72, 199-301.

Maharachchikumbura SSN, Hyde KD, Jones EBG, Mckenzie EHC et al. 2016 - Families of Sordariomycetes. Fungal Diversity, 79, 1-317.

Malloch D. 1989 - An undescribed species of Leucosphaerina. Studies in Mycology 31, 107-111.

Manamgoda DS, Cai L, McKenzie EHC, Crous PW et al. 2012 - A phylogenetic and taxonomic reevaluation of the Bipolaris - Cochliobolus - Curvularia complex. Fungal Diversity 56, 131144.

Mapook A, Hyde KD, McKenzie EH, Jones EG et al. 2020 - Taxonomic and phylogenetic contributions to fungi associated with the invasive weed Chromolaena odorata (Siam weed). Fungal Diversity 101, 1-175.

Marin-Felix Y, Groenewald JZ, Cai L, Chen Q et al. 2017 - Genera of phytopathogenic fungi: GOPHY 1. Studies Mycology 86, 99-216.

Marin-Felix Y, Guarro J, Cano-Lira JF, García D. et al. 2018 Melanospora (Sordariomycetes, Ascomycota) and its relatives. MycoKeys 44, 81-122.

Marin-Felix Y, Stchigel AM, Miller AN, Guarro J, Cano-Lira JF. 2015 - A re-evaluation of the genus Myceliophthora (Sordariales, Ascomycota): its segregation into four genera and description of Corynascus fumimontanus sp. nov. Mycologia 107, 619-32.

Matsumura M, Kato W, Hashimoto A, Takahashi YS et al. 2018 - Crassiperidium (Pleosporales, Dothideomycetes), a new ascomycetous genus parasitic on Fagus crenatain Japan. Mycosphere 9, 1256-1267. 
Matsushima T. 2001 - Matsushima Mycological Memoirs 9. Matsushima Mycological Memoirs $10,1-214$.

May TW, Redhead SA, Bensch K, Hawksworth DL et al. 2019 Chapter F of the International Code of Nomenclature for algae, fungi, and plants as approved by the 11th International Mycological Congress, San Juan, Puerto Rico, July 2018. IMA fungus. 10, 1-4.

Miller AN, Huhndorf SM. 2004 - A natural classification of Lasiosphaeria based on nuclear LSU rDNA sequences. Mycological Research 108, 26-34.

Minnis AM, Kennedy AH, Grenier DB, Palm ME, Rossman AY. 2012 - Phylogeny and taxonomic revision of the Planistromellaceae including its coelomycetous anamorphs: contributions towards a monograph of the genus Kellermania. Persoonia 29, 11-28.

Minter D, Caine T. 1980 - Trichocrea Marchal, Fujimyces and Ebollia gen. nov. Transactions of the British. Mycological Society 74, 434-437.

Mongkolsamrit S, Noisripoom W, Arnamnart N. et al. 2020 - Resurrection of Paraisaria in the Ophiocordycipitaceae with three new species from Thailand. Mycol Progress 18, 1213-1230

Mongkolsamrit S, Noisripoom W, Thanakitpipattana D, Wutikhun T et al. 2018 - Disentangling cryptic species with isaria-like morphs in Cordycipitaceae. Mycologia 110, 230-57.

Monkai J, Liu JK, Boonmee S, Chomnunti P et al. 2013 - Planistromellaceae (Botryosphaeriales). Cryptogamie Mycologie 34, 45-77.

Moore RT. 1962 - Hiospira, a new genus of the Helicosporae. Transaction of the British Mycological Society 45, 143-146.

Morin L, Shivas RG, Piper MC, Tan YP. 2010 - Austropleospora osteospermi gen. et sp. nov. and its host specificity and distribution on Chrysanthemoides monilifera ssp. rotundata in Australia. Fungal Diversity 40, 65-74.

Muggia L, Kopun T, Ertz D. 2015 - Phylogenetic placement of the lichenicolous, anamorphic genus Lichenodiplis and its connection to Muellerella-like teleomorphs. Fungal Biology 119, 1115-1128.

Muntañola M. 1960 - Algunos Hyphomycetes criticos. Notas y descripciones. Lilloa 30, 165-232.

Norphanphoun C, Hongsanan S, Doilom M, Bhat DJ et al. 2016 - Lamproconiaceae fam. nov. to accommodate Lamproconium desmazieri. Phytotaxa 270, 89-102.

Otálora MAG, Jørgensen PM, Wedin M. 2014 - A revised generic classification of the jelly lichens, Collemataceae. Fungal Diversity 64, 275-293.

Parbery DG. 1969 - Amorphotheca resinae gen. nov., sp. nov.: The perfect state of Cladosporium resinae. Australian Journal of Botany 17, 331-357.

Parker AK, Reid J. 1969 - The genus Rhabdocline Syd. Canadian Journal of Botany 47, 15331545.

Peterson SW, Jurjevic Z, Bills GF, Stchigel AM et al. 2010 - Genus Hamigera, six new species and multilocus DNA sequence based phylogeny. Mycologia 102, 847-64.

Petrak F. 1928 - Über Bagnisiopsis und verwandte Gattungen. Hedwigia 68, 251-290.

Petrak F. 1953 - Cyclopeltis n. gen., eine neue Gattung der Polystomellaceen. Sydowia 7, 370-374.

Petrak F. 1958 - Singeriella n. gen., eine neue, mit den Entopeltaceen verwandte Gattung aus den Hochgebirgen Perus. Sydowia 12, 252-257.

Phillips AJL, Alves A, Abdollahzadeh J, Slippers B et al. 2013 - The Botryosphaeriaceae: genera and species known from culture. Studies in Mycology 76, 51-167.

Phillips AJL, Alves A. 2009 - Taxonomy, phylogeny, and epitypification of Melanops tulasnei, the type species of Melanops. Fungal Diversity 38, 155-166.

Phookamsak R, Hyde KD, Jeewon R, Bhat DJ et al. 2019 - Fungal Diversity notes 929-1035: taxonomic and phylogenetic contributions on genera and species of fungi. Fungal Diversity 95, 1-273.

Phookamsak R, Liu JK, Chukeatirote E, McKenzie EHC, Hyde KD. 2013 - Phylogeny and morphology of Leptosphaerulina saccharicola sp. nov. and Pleosphaerulina oryzae and relationships with Pithomyces. Cryptogamie Mycologie 34, 303-319. 
Phookamsak R, Liu JK, McKenzie EHC, Manamgoda DS et al. 2014 - Revision of Phaeosphaeriaceae. Fungal Diversity 68, 159-238.

Phookamsak R, Lu YZ, Hyde KD, Jeewon R et al. 2018 - Phylogenetic characterization of two novel Kamalomyces species in Tubeufiaceae (Tubeufiales). Mycological Progress 17, 647-60.

Phookamsak R, Norphanphoun C, Tanaka K, Dai DQ et al. 2015 - Towards a natural classification of Astrosphaeriella-like species; introducing Astrosphaeriellaceae and Pseudoastrosphaeriellaceae fam. nov. and Astrosphaeriellopsis, gen. nov. Fungal Diversity 74, 143-197.

Phukhamsakda C, Ariyawansa HA, Phillips AJ, Wanasinghe DN et al. 2016 - Additions to Sporormiaceae: Introducing two novel genera, Sparticola and Forliomyces, from Spartium. Cryptogamie Mycologie 37, 75-97.

Phukhamsakda C, Ariyawansa HA, Phookamsak R, Chomnunti P et al. 2015 - Muriphaeosphaeria galatellae gen. et sp. nov. in Phaeosphaeriaceae (Pleosporales). Phytotaxa 227, 55-65.

Phukhamsakda C, McKenzie EH, Phillips AJ, Jones EG et al. 2020 - Microfungi associated with Clematis (Ranunculaceae) with an integrated approach to delimiting species boundaries. Fungal Diversity 102, 1-203.

Pinruan U, Sakayaroj J, Hyde KD, Jones EB. 2008 - Thailandiomyces bisetulosus gen. et sp. nov. (Diaporthales, Sordariomycetidae, Sordariomycetes) and its anamorph Craspedodidymum, is described based on nuclear SSU and LSU rDNA sequences. Fungal Diversity 29, 89-98.

Pitt JI, Lantz H, Pettersson OV, Su-lin LL. 2013 - Xerochrysium gen. nov. and Bettsia, genera encompassing xerophilic species of Chrysosporium. IMA Fungus 4(2), 229-241.

Põldmaa K, Larsson E, Kõljalg U. 2000 - Phylogenetic relationships in Hypomyces and allied genera, with emphasis on species growing on wood-decaying homobasidiomycetes. Canadian Journal of Botany 77, 1756-1768.

Potebnia A. 1910 - Beitr€age zur Micromycetenflora Mittel-Russlands (Gouv Kursk und Charkow). Annales Mycologici 8, 42-93.

Quaedvlieg W, Verkley GJM, Shin HD, Barreto RW et al. 2013 - Sizing up Septoria. Studies in Mycology 75, 307-390.

Quandt CA, Kepler RM, Gams W, Araújo JP et al. 2014 - Phylogenetic-based nomenclatural proposals for Ophiocordycipitaceae (Hypocreales) with new combinations in Tolypocladium. IMA Fungus 5, 121-34.

Ram C. 1971 - Timber-attacking fungi from the state of Maranhao, Brazil. New species of Pyrenomycetes XI. Nova Hedwigia 21, 225-230.

Ramaley AW, Barr ME. 1995 - New dictyosporous species from leaves of Agavaceae. Mycotaxon 54, 75-90.

Ramaley AW, Barr ME. 1997 - Syncarpella ribis sp. nov. and its anamorph, Syntholus ribis gen. et sp. nov. Mycotaxon 65, 499-506.

Ramaley RA. 1997 - Barrina, a new genus with polysporous asci, Mycologia 89, 962-966.

Ranghoo VM, Hyde KD. 1998 - Ascomycetes from freshwater habitats: Ascolacicola aquatica gen. et sp. nov. and a new species of Ascotaiwania from wood submerged in a reservoir in Hong Kong. Mycologia 90, 1055-1062.

Rappaz F. 1992 - Phomatospora berkeleyi, P. arenaria and their Sporothrix anamorphs. Mycotaxon 45, 323-330.

Réblová M, Fournier J, Stepánek V. 2016c - Two new lineages of aquatic ascomycetes: Atractospora gen. nov. and Rubellisphaeria gen. et sp. nov., and a sexual morph of Myrmecridium montsegurinum sp. nov. Mycological Progress15, 1-18.

Réblová M, Hernández-Restrepo M, Fournier J, Nekvindová J. 2020a New insights into the systematics of Bactrodesmium and its allies and introducing new genera, species and morphological patterns in the Pleurotheciales and Savoryellales (Sordariomycetes). Studies in Mycology 95, 415-466. 
Réblová M, Jaklitsch WM, Reblova K, Štěpánek V. 2015 - Phylogenetic reconstruction of the Calosphaeriales and Togniniales using five genes and predicted RNA secondary structures of ITS, and Flabellascus tenuirostris gen. et sp. nov. PloS one 10, e0144616.

Reblová M, Miller A, Reblová K, Štepánek V. 2018 - Phylogenetic classification and generic delineation of Calyptosphaeria gen. nov., Lentomitella, Spadicoides and Torrentispora (Sordariomycetes). Studies in Mycology 89, 1-62.

Réblová M, Miller AN, Rossman AY, Seifert KA et al. 2016a - Recommendations for competing sexual-asexually typified generic names in Sordariomycetes (except Diaporthales, Hypocreales, and Magnaporthales). IMA Fungus 7, 131-153.

Réblová M, Mostert L, Gams W, Crous PW. 2004 - New genera in the Calosphaeriales: Togniniella and its anamorph Phaeocrella, and Calosphaeriophora as anamorph of Calosphaeria. Studies in Mycology 50, 533-50.

Réblová M, Nekvindová J, Fournier J, Miller AN 2020b - Delimitation, new species and teleomorph-anamorph relationships in Codinaea, Dendrophoma, Paragaeumannomyces and Striatosphaeria (Chaetosphaeriaceae). MycoKeys 74, 17-74.

Réblová M, Seifert KA, Fournier J, Stepánek V. 2012 - Phylogenetic classification of Pleurothecium and Pleurotheciella gen. nov. and its dactylaria-like anamorph (Sordariomycetes) based on nuclear ribosomal and protein-coding genes. Mycologia 104, 1299-1314.

Réblová M, Seifert KA, Fournier J, Štěpánek V. 2016b - Newly recognised lineages of perithecial ascomycetes: the new orders Conioscyphales and Pleurotheciales. Persoonia 37, 57-81.

Réblová M, Seifert KA. 2007 - A new fungal genus, Teracosphaeria, with a phialophora-like anamorph (Sordariomycetes, Ascomycota). Mycological Research 111, 287-98.

Réblová M, Štěpánek V, Schumacher RK. 2014 - Xylochrysis lucida gen. et sp. nov., a new lignicolous ascomycete (Sordariomycetidae) with holoblastic conidiogenesis. Mycologia 106, 564-572.

Réblová M, Štepánek V. 2018 - Introducing the Rhamphoriaceae fam. nov. (Sordariomycetes), two new genera and new life histories for taxa with Phaeoisaria- and Idriella-like anamorphs. Mycologia 110, 750-770.

Réblová M, Winka K. 2000 - Phylogeny of Chaetosphaeria and its anamorphs based on morphological and molecular data. Mycologia 92, 939-954.

Réblová M. 1999 Studies in Chaetosphaeria sensu lato. IV. Crassochaeta gen. nov., a new lignicolous genus of the Trichosphaeriaceae. Mycotaxon 71, 45-67.

Réblová M. 2006 - Molecular systematics of Ceratostomella sensu lato and morphologically similar fungi. Mycologia 98, 68-93.

Réblová M. 2007 - Barbatosphaeria gen. et comb. nov., a new genus for Calosphaeria barbirostris. Mycologia 99, 723-732.

Rehner SA, Minnis AM, Sung GH, Luangsa-ard JJ. et al. 2011 - Phylogeny and systematics of the anamorphic, entomopathogenic genus Beauveria. Mycologia 103, 1055-1073.

Reusser FA. 1964 - Übcr einige Arten der Gattung Guignardia Viala et Ravaz. Journal of Phytopathology 51, 205-240.

Rossman A, Udayanga D, Castlebury LA, Hyde KD. 2013 - (2141) proposal to conserve the name Phomopsis citri H. S. Fawc. (Diaporthe citri), with a conserved type, against Phomopsis citri (Sacc.) Traverso \& Spessa (Ascomycota: Diaporthales: Diaporthaceae). Taxon 62, 627-627.

Rossman AY, Adams GC, Cannon PF, Castlebury LA et al. 2015a - Recommendations of generic names in Diaporthales competing for protection or use. IMA Fungus 6, 145-54.

Rossman AY, Allen WC, Braun U, Castlebury LA et al. 2016 - Overlooked competing asexual and sexually typified generic names of Ascomycota with recommendations for their use or protection. IMA Fungus 7, 289-308.

Rossman AY, Crous PW, Hyde KD, Hawksworth DL et al. 2015b - Recommended names for pleomorphic genera in Dothideomycetes. IMA Fungus 6, 507-523. 
Rossman AY, Samuels GJ, Rogerson CT et al. 1999 - Genera of Bionectriaceae, Hypocreaceae and Nectriaceae (Hypocreales, Ascomycetes). Studies in Mycology 42, 1-248.

Samerpitak K, Van der Linde E, Choi H et al. 2014 - Taxonomy of Ochroconis, genus including opportunistic pathogens on humans and animals. Fungal Diversity 65, 89-126.

Samuels GJ, Barr ME. 1997 - Notes on and additions to the Niessliaceae (Hypocreales). Canadian Journal of Botany 75, 2165-2176.

Sandoval-Denis M, Gené J, Sutton DA, Cano-Lira JF et al. 2016 - Redefining Microascus, Scopulariopsis and allied genera. Persoonia 36, 1-36.

Sanoamuang N, Jitjak W, Rodtong S, Whalley JS. 2013 - Gelatinomyces siamensis gen. sp. nov. (Ascomycota, Leotiomycetes, incertae sedis) on bamboo in Thailand. IMA Fungus 4, 71-80.

Saxena RK, Wijayawardene NN, Dai DQ, Hyde KD, Kirk PM. 2021 - Diversity in fossil fungal spores. Mycosphere (submitted).

Schoch CL, Crous PW, Groenewald JZ, Boehm EWA et al. 2009 - A class-wide phylogenetic assessment of Dothideomycetes. Studies in Mycology 64, 1-15.

Schoch CL, Crous PW, Wingfield MJ, Wingfield BD. 2000 - Phylogeny of Calonectria and selected hypocrealean genera with cylindrical macroconidia. Studies in Mycology 45, 45-62.

Seifert K, Morgan-Jones G, Gams W, Kendrick B. 2011 - The genera of hyphomycetes. CBSKNAW Fungal Biodiversity Centre, Utrecht, the Netherlands.

Senanayake IC, Crous PW, Groenewald JZ, Maharachchikumbura SS et al. 2017a - Families of Diaporthales based on morphological and phylogenetic evidence. Studies in Mycology 86, 217-96.

Senanayake IC, Maharachchikumbura SS, Hyde KD, Bhat JD et al. 2015 - Towards unraveling relationships in Xylariomycetidae (Sordariomycetes). Fungal Diversity 73, 73-144.

Senanayake IC, Maharachchikumbura SSN, Jeewon R, Promputtha I et al. 2017b Morphophylogenetic study of Sydowiellaceae reveals several new genera. Mycosphere 8, 172-217.

Senwanna C, Wanasinghe DN, Bulgakov TS, Wang Y et al. 2019 - Towards a natural classification of Dothidotthia and Thyrostroma in Dothidotthiaceae (Pleosporineae, Pleosporales). Mycosphere 10, 701-738.

Sharma R, Gräser Y, Singh SK. 2013 - Auxarthronopsis, a new genus of Onygenales isolated from the vicinity of Bandhavgarh National Park, India. IMA Fungus 4, 89-102.

Sharma R, Shouche YS. 2020 - Diversity of Onygenalean fungi in keratin-rich habitats of Maharashtra (India) and description of three novel taxa. Mycopathologia 185, 67-85.

Shear CL. 1923 - Life histories and undescribed genera and species of fungi. Mycologia 15, 12031.

Sivanesan A. 1981 - Balladynopsis, Balladynocallia and Alina. Mycological Papers 146, 1-38.

Sivanesan A. 1983 - Studies on ascomycetes. Transactions of the British Mycological Society 81, 313-332.

Sivanesan A. 1984 - The bitunicate ascomycetes and their anamorphs. J. Cramer, Vaduz.

Slippers B, Boissin E, Phillips AJL, Groenewald JZ et al. 2013 - Phylogenetic lineages in the Botryosphaeriales: A systematic and evolutionary framework. Studies in Mycology 76, 3149.

Sogonov MV, Castlebury LA, Rossman AY, Mejía LC, White JF. 2008 - Leaf-inhabiting genera of the Gnomoniaceae, Diaporthales. Studies in Mycology 62, 1-77.

Stadler M, Fournier J, Læssøe T, Decock C et al. 2010 - Ruwenzoria, a new genus of the Xylariaceae from Central Africa. Mycological Progress 9, 169-79.

$\mathrm{Su}$ H, Hyde KD, Maharachchikumbura SS, Ariyawansa HA et al. 2016 - The families Distoseptisporaceae fam. nov., Kirschsteiniotheliaceae, Sporormiaceae and Torulaceae, with new species from freshwater in Yunnan Province, China. Fungal Diversity 80, 375-409.

Suetrong S, Klaysuban A, Sakayaroj J, Preedanon S et al. 2015 - Tirisporellaceae, a new family in the order Diaporthales (Sordariomycetes, Ascomycota). Cryptogamie Mycologie 36, 319-330. 
Sullivan RF, Bills GF, Hywel-Jones NL, White JFJ. 2000 - Hyperdermium: a new clavicipitalean genus for some tropical epibionts of dicotyledonous plants. Mycologia 92, 908-918.

Sutton BC. (1980) The Coelomycetes. Fungi imperfecti with pycnidia, acervuli and stromata. Commonwealth Mycological Institute, Kew.

Swart HJ. 1971 - Australian leaf-inhabiting fungi: I. Two species of Vizella. Transactions of the British Mycological Society 57, 455-64.

Sydow H, Petrak F. 1922 - Ein Beitrag zur Kenntnis der Pilzflora Nordamerikas, insbesondere der nordwestlichen Staaten. Annales Mycologici 20, 178-218.

Tanaka E, Tanaka C, Tsuda M, Gafur A. 2002 - Heteroepichloë, gen. nov. (Clavicipitaceae; Ascomycotina) on bamboo plants in East Asia. Mycoscience 43, 87-93.

Tanaka K, Endo M, Hirayama K, Okane I, Hosoya T, Sato T. 2011 - Phylogeny of Discosia and Seimatosporium, and introduction of Adisciso and Immersidiscosia genera nova. Persoonia 26, 85-98.

Tanaka K, Hirayama K, Yonezawa H, Sato G et al. 2015 - Revision of the Massarineae (Pleosporales, Dothideomycetes). Studies in Mycology 82, 75-136.

Tanaka K, Hirayama K, Yonezawa H, Hatakeyama S et al. 2009 - Molecular taxonomy of bambusicolous fungi: Tetraplosphaeriaceae, a new pleosporalean family with Tetraploa-like anamorphs. Studies in Mycology 64, 175-209.

Tanaka K, Ooki Y, Hatakeyama S, Harada Y, Barr ME. 2005 - Pleosporales in Japan (5): Pleomassaria, Asteromassaria, and Splanchnonema. Mycoscience 46, 248-260.

Tedersoo L, Sánchez-Ramírez S, Koljalg U, Bahram M et al. 2018 - High-level classification of the Fungi and a tool for evolutionary ecological analyses. Fungal Diversity 90, 135-159.

Thambugala KM, Chunfang Y, Camporesi E, Bahkali AH et al. 2015a - Pseudodidymosphaeria gen. nov. in Massarinaceae. Phytotaxa 231, 271-282.

Thambugala KM, Daranagama DA, Phillips AJ, Bulgakov TS. 2017a - Microfungi on Tamarix. Fungal Diversity 82, 239-306.

Thambugala KM, Hyde KD, Tanaka K, Tian Q et al. 2015b - Towards a natural classification and backbone tree for Lophiostomataceae, Floricolaceae and Amorosiaceae fam. nov. Fungal Diversity 74, 199-266.

Thambugala KM, Wanasinghe DN, Phillips AJL, Camporesi E et al. 2017b - Mycosphere notes 150: Grass (Poaceae) inhabiting Dothideomycetes. Mycosphere 8, 697-796.

Tian Q, Liu JK, Hyde KD, Wanasinghe DN et al. 2015 - Phylogenetic relationships and morphological reappraisal of Melanommataceae (Pleosporales). Fungal Diversity 74, 267324.

Tibell L. 1991 - The anamorph of Tylophoron moderatum. Mycological research 95, 290-294.

Tibpromma S, Hyde KD, Bhat JD, Mortimer PE et al. 2018a - Identification of endophytic fungi from leaves of Pandanaceae based on their morphotypes and DNA sequence data from southern Thailand. MycoKeys 18, 25-67.

Tibpromma S, Hyde KD, Jeewon R, Maharachchikumbura SSN et al. 2017 - Fungal diversity notes 491-602: taxonomic and phylogenetic contributions to fungal taxa. Fungal Diversity 83, 1261.

Tibpromma S, Hyde KD, McKenzie EH, Bhat DJ et al. 2018b - Fungal diversity notes 840-928: micro-fungi associated with Pandanaceae. Fungal Diversity 93, 1-60.

Ueda S. 1994 - A new Cercophora with a Chrysosporium-like anamorph. Mycoscience 35, 28790.

Upadhyay HP, Kendrick WB. 1975 - Prodromus for a revision of. Ceratocystis (Microascales, Ascomycetes) and its conidial states. Mycologia 67, 798-805.

Valenzuela-Lopez N, Cano-Lira JF, Guarro J, Sutton DA et al. 2017 - Coelomycetous Dothideomycetes with emphasis on the families Cucurbitariaceae and Didymellaceae. Studies Mycology 90, 1-69. 
Van der Linde JA, Six DL, De Beer WZ, Wingfield MJ, Roux J. 2016 - Novel ophiostomatalean fungi from galleries of Cyrtogenius africus (Scolytinae) infesting dying Euphorbia ingens. Antonie van Leeuwenhoek 109, 589-601.

Verkley GJM, Dukik K, Renfurm R, Göker M et al. 2014 - Novel genera and species of Coniothyrium-like fungi in Montagnulaceae (Ascomycota). Persoonia 32, 25-51.

Verkley GJM. 1999 - A monograph of the genus Pezicula and its anamorphs. Studies in Mycology 44, 1-180.

Videira SI, Groenewald JZ, Braun U, Shin HD, Crous PW. 2016 - All that glitters is not Ramularia. Studies in Mycology 83, 49-163.

Videira SIR, Groenewald JZ, Nakashima C, Braun U et al. 2017 - Mycosphaerellaceae - chaos or clarity? Studies in Mycology 87, 257-421.

Viljoen CD, Wingfield MJ, Jacobs K, Wingfield BD. 2000 - Cornuvesica, a new genus to accommodate Ceratocystiopsis falcata. Mycological Research 104, 365-367.

Voglmayr H, Castlebury LA, Jaklitsch WM. 2017 - Juglanconis gen. nov. on Juglandaceae, and the new family Juglanconidaceae (Diaporthales). Persoonia 38, 136-155.

Voglmayr H, Gardiennet A, Jaklitsch WM. 2016 - Asterodiscus and Stigmatodiscus, two new apothecial Dothideomycete genera and the new order Stigmatodiscales. Fungal Diversity 80, 271-284.

Voglmayr H, Jaklitsch WM, Tello S 2020 - Mycosphaerangium and Neomelanconium (Cenangiaceae) are closest relatives: phylogenetic relationships, morphology and a new species. Mycological Progress 19, 1329-1352.

Voglmayr H, Jaklitsch WM. 2017 - Corynespora, Exosporium and Helminthosporium revisited New species and generic reclassification. Studies in Mycology 87, 43-76.

Voglmayr H, Rossman AY, Castlebury LA, Jaklitsch WM. 2012 - Multigene phylogeny and taxonomy of the genus Melanconiella (Diaporthales). Fungal Diversity 57, 1-44.

Von Arx JA. 1987 - A re-evaluation of the Eurotiales. Persoonia 13, 273-300.

Wäli PP, Huhtinen S, Pino-Bodas R, Stenroos S. 2014 - Three common bryophilous fungi with meristematic anamorphs and phylogenetic alliance to Teratosphaeriaceae, Capnodiales. Fungal Biology 118, 956-969.

Wanasinghe DN, Hyde KD, Jeewon R, Crous PW. 2017a - Phylogenetic revision of Camarosporium (Pleosporineae, Dothideomycetes) and allied genera. Studies in Mycology 87, 207-256.

Wanasinghe DN, Jones EBG, Camporesi E, Boonmee S et al. 2014 - An exciting novel member of Lentitheciaceae in Italy from Clematis vitalba. Cryptogamie, Mycologie. 35, 323-337.

Wanasinghe DN, Jones EG, Camporesi E, Mortimer PE et al. 2015 - The genus Murispora. Cryptogamie Mycologie 36, 419-448.

Wanasinghe DN, Phookamsak R, Jeewon R, Li WJ et al. 2017b - Fenestellaceae with descriptions of new Fenestella species and Neocucurbitaria gen. nov. Mycosphere 8, 397-414.

Wanasinghe DN, Phukhamsakda C, Hyde KD, Jeewon R et al. 2018 - Fungal diversity notes 709839: taxonomic and phylogenetic contributions to fungal taxa with an emphasis on fungi on Rosaceae. Fungal Diversity 89, 1-236.

Wanasinghe DN, Wijayawardene NN, Xu J, Cheewangkoon R, Mortimer PE. 2020 - Taxonomic novelties in Magnolia-associated pleosporalean fungi in the Kunming Botanical Gardens (Yunnan, China). Plos one 15, e0235855.

Wang Y, Hyde KD, Mckenzie EHC, Jiang YL et al. 2015 - Overview of Stachybotrys (Memnoniella) and current species status. Fungal Diversity 71, 17-83.

Wedin M, Otalora MAG, Jørgensen PM. 2017 - The genus Paracollema validated (Collemataceae, Peltigerales, Lecanoromycetes). Studies in Fungi 2, 208-209.

Wendt L, Sir EB, Kuhnert E, Heitkämper S et al. 2017 - Resurrection and emendation of the Hypoxylaceae, recognised from a multigene phylogeny of the Xylariales. Mycological Progress 17, 115-154. 
Whalley AJ, Gareth-Jones EB, Hyde KD, Laessoe T. 2000 - Halorosellinia gen. nov. to accommodate Hypoxylon oceanicum, a common mangrove species. Mycological Research 104, 368-374.

White Jr JF, Bacon CW, Hywel-Jones NL, Spatafora JW. 2003 - Clavicipitalean fungi: evolutionary biology, chemistry, biocontrol and cultural impacts (Vol. 19). CRC Press.

White Jr JF, Glenn AE. 1994 - A study of two fungal epibionts of grasses: structural features, host relationships, and classification in the genus Myriogenospora (Clavicipitales). American Journal of Botany 81, 216-223.

Wijayawardene DNN, McKenzie EHC, Hyde KD 2012 - Towards incorporating anamorphic fungi in a natural classification checklist and notes for 2011. Mycosphere 3,157-228.

Wijayawardene NN, Crous PW, Kirk PM, Hawksworth DL et al. 2014a - Including proposals for the protection or suppression of generic names. Fungal Diversity 69, 1-55.

Wijayawardene NN, Hyde KD, Al-Ani LK, Tedersoo L et al. 2020 - Outline of Fungi and funguslike taxa. Mycosphere 11, 1060-1456.

Wijayawardene NN, Hyde KD, Camporesi E, Bhat DJ et al. 2014b - Homortomyces tamaricis sp. nov. and convergent evolution of Homortomyces and Stilbospora. Phytotaxa 176, 156-163.

Wijayawardene NN, Hyde KD, Lumbsch HT, Liu JK et al. 2018 - Outline of Ascomycota: 2017. Fungal Diversity 88, 167-263.

Wijayawardene NN, Hyde KD, Rajeshkumar KC, Hawksworth DL et al. 2017a - Notes for genera: Ascomycota. Fungal Diversity 86, 1-594.

Wijayawardene NN, Hyde KD, Tibpromma S, Wanasinghe DN et al. 2017b - Towards incorporating asexual fungi in a natural classification: checklist and notes 2012- 2016. Mycosphere 8, $1457-1555$.

Wijayawardene NN, Hyde KD, Wanasinghe DN, Papizadeh M et al. 2016 - Taxonomy and phylogeny of dematiaceous coelomycetes. Fungal Diversity 77, 1-316.

Wijesinghe SN, Wanasinghe DN, Maharachchikumbura SS, Wang Y, Al-Sadi AM, Hyde KD. 2020 - Bimuria omanensis sp. nov. (Didymosphaeriaceae, Pleosporales) from Oman. Phytotaxa 449(2), 97-108.

Wijesinghe SN, Wang Y, Camporesi E, Wanasinghe DN, Boonmee S, Hyde KD. 2020 - A new genus of Bambusicolaceae (Pleosporales) on Corylus avellana (Fagales) from Italy. Biodiversity data journal 8,e55957.

Wikee S, Udayanga D, Crous PW, Chukeatirote E et al. 2011 - Phyllosticta-an overview of current status of species recognition. Fungal Diversity 51, 43-61.

Woudenberg JHC, Sandoval-Denis M, Houbraken J, Seifert KA et al. 2017 - Cephalotrichum and related synnematous fungi with notes on species from the built environment. Studies in Mycology 88, 137-159.

Yang CL, Xu XL, Wanasinghe DN, Jeewon R et al. 2019 - Neostagonosporella sichuanensis gen. et sp. nov. (Phaeosphaeriaceae, Pleosporales) on Phyllostachys heteroclada (Poaceae) from Sichuan Province, China. MycoKeys 46, 119-150.

Yang H, Hyde KD, Karunarathna SC, Deng C, et al. 2018a - New species of Camptophora and Cyphellophora from China, and first report of sexual morphs for these genera. Phytotaxa 343(2), 149-159.

Yang J, Maharachchikumbura SSN, Bhat DJ, Hyde KD et al. 2016 - Fuscosporellales, a new order of aquatic and terrestrial Hypocreomycetidae (Sordariomycetes). Cryptogamie, Mycologie 37, 449-475.

Yang Q, Fan XL, Du Z, Tian CM. 2018b - Diaporthosporellaceae, a novel family of Diaporthales (Sordariomycetes, Ascomycota). Mycoscience 59, 229-35.

Yilmaz N, Visagie CM, Houbraken J, Frisvad JC, Samson RA. 2014 - Polyphasic taxonomy of the genus Talaromyces. Studies in Mycology 78, 175-341.

Zalasky H, Nawawi A, Ting WP, Tai LH. 1971 - Dolabra nepheliae and its imperfect state associated with canker of Nephelium lappaceum and $N$. mutabile. Canadian Journal of Botany 49, 559-61. 
Zare R, Gams W, Evans HC. 2001 - A revision of Verticillium section Prostrata. V. The genus Pochonia, with notes on Rotiferophthora. Nova Hedwigia 73, 51-86.

Zelski SE, Raja HA, Miller AN, Shearer CA. 2011 - Chaetorostrum quincemilensis, gen. et sp. nov., a new freshwater ascomycete and its Taeniolella-like anamorph from Peru. Mycosphere 2, 593-600.

Zhang H, Dong W, Hyde KD, Maharachchikumbura SS et al. 2017 - Towards a natural classification of Annulatascaceae-like taxa: introducing Atractosporales ord. nov. and six new families. Fungal Diversity 85, 75-110.

Zhang N, Luo J, Rossman AY, Aoki T et al. 2016 - Generic names in Magnaporthales. IMA Fungus 7, 155-159.

Zhang Y, Crous PW, Schoch CL, Bahkali AH, Guo LD, Hyde KD. 2011 - A molecular, morphological and ecological re-appraisal of Venturiales-a new order of Dothideomycetes. Fungal Diversity 51, 249-277.

Zhuang WY. 1988 - A monograph of the genus Unguiculariopsis (Leotiaceae, Encoelioideae). Mycotaxon 32, 1-8. 